\title{
NEUTRINO AND ANTINEUTRINO INCLUSIVE \\ CHARGED-CURRENT CROSS SECTION MEASUREMENT \\ WITH THE MINOS NEAR DETECTOR
}

by

\section{Debdatta Bhattacharya}

B.Sc.(HONS), Physics, Presidency College, Calcutta, 2000

M.Sc., Physics, Indian Institute of Technology, Kharagpur, 2002

M.S., Physics, University of Pittsburgh, 2004

Submitted to the Graduate Faculty of the School of Arts and Sciences in partial fulfillment of the requirements for the degree of Doctor of Philosophy

University of Pittsburgh

2009 


\section{UNIVERSITY OF PITTSBURGH \\ FACULTY OF ARTS AND SCIENCES}

This dissertation was presented

by

Debdatta Bhattacharya

It was defended on

February $27^{\text {th }} 2009$

and approved by

Dr. Donna Naples, Associate Professor, Department of Physics and Astronomy

Dr. Arthur Kosowsky, Associate Professor, Department of Physics and Astronomy

Dr. Adam Leibovich, Associate Professor, Department of Physics and Astronomy

Dr. Vittorio Paolone, Associate Professor, Department of Physics and Astronomy

Dr. George Sparling, Department of Mathematics

Dissertation Director: Dr. Donna Naples, Associate Professor, Department of Physics and Astronomy 
Copyright (C) by Debdatta Bhattacharya 2009 


\title{
NEUTRINO AND ANTINEUTRINO INCLUSIVE CHARGED-CURRENT CROSS SECTION MEASUREMENT WITH THE MINOS NEAR DETECTOR
}

\author{
Debdatta Bhattacharya, PhD
}

University of Pittsburgh, 2009

This thesis presents the measurement of energy dependence of the neutrino-nucleon inclusive charged current cross section on an isoscalar target in the range 3-50 GeV for neutrinos and 5$50 \mathrm{GeV}$ energy range for antineutrinos. The data set was collected with the MINOS Near Detector using the wide band NuMI beam at Fermilab. The size of the charged current sample is $1.94 \times 10^{6}$ neutrino events and $1.60 \times 10^{5}$ antineutrino events.

The flux has been extracted using a low hadronic energy sub-sample of the charged current events. The energy dependence of the cross section is obtained by dividing the charged current sample with the extracted flux. The neutrino and antineutrino cross section exhibits a linear dependence on energy at high energy but shows deviations from linear behavior at low energy. We also present a measurement of the ratio of antineutrino to neutrino inclusive cross section. 


\section{TABLE OF CONTENTS}

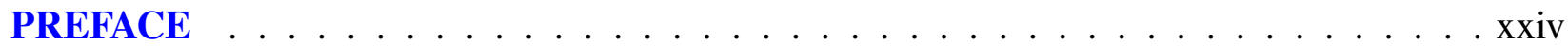

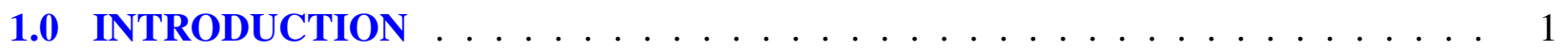

1.1 Standard model and neutrinos . . . . . . . . . . . . . . . 3

1.2 Neutrino charged current cross section . . . . . . . . . . . . . 6

1.2.1 Kinematic Quantities ................... 6

1.2.2 Neutrino Interaction cross section . . . . . . . . . . . . . 8

1.2.2.1 Quasi Elastic Interactions . . . . . . . . . . . . . . . 9

1.2.2.2 Resonance Interactions . . . . . . . . . . . . . . . . . 9

1.2.2.3 Deep Inelastic Scattering . . . . . . . . . . . . . . . 11

2.0 THE NUMI BEAM AND MINOS DETECTORS . . . . . . . . . . . . . . . . 17

2.1 NuMi Beam . . . . . . . . . . . . . . . . . . . 17

2.2 Near Detector . . . . . . . . . . . . . . . . . . 21

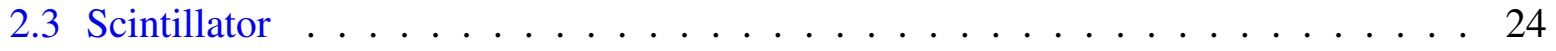

2.4 Photo Multiplier Tubes . . . . . . . . . . . . . . . . . . . 25

2.5 Magnetic Field . . . . . . . . . . . . . . . . . . . . 28

2.6 Readout and Data Acquisition . . . . . . . . . . . . . . . 30

2.6.1 Electronic Readout . . . . . . . . . . . . . . . . 32

2.6 .2 Data Acquisition . . . . . . . . . . . . . . . 34

2.7 Far Detector . . . . . . . . . . . . . . . . . 35

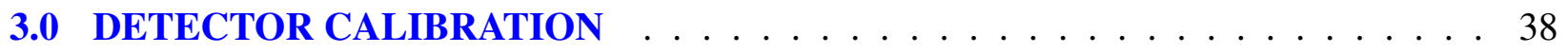

3.1 Calibration Detector . . . . . . . . . . . . . . 38

3.2 Calibration Procedure . . . . . . . . . . . . . . . 41 
3.2.1 Gain drift and Linearity Correction . . . . . . . . . . . . . . . . 41

3.2 .2 Intrastrip Correction . . . . . . . . . . . . . . . . . . 42

3.2 .3 Strip-to-Strip Calibration . . . . . . . . . . . . . . . . . 42

3.2.4 Muon Energy Equivalent(M.E.U.) Calibration . . . . . . . . . . . . . 42

3.2 .5 Absolute Energy Scale . . . . . . . . . . . . . . . . . . . . . . 43

3.3 Muon Energy Scale . . . . . . . . . . . . . . . . . . . . . . 45

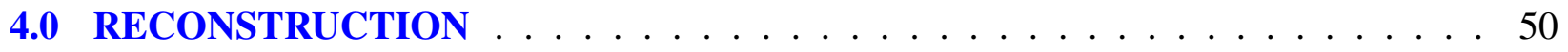

4.0 .1 Hit Strip formation $\ldots \ldots \ldots \ldots \ldots \ldots \ldots$

4.0 .2 Slice formation $\ldots \ldots \ldots \ldots \ldots \ldots$

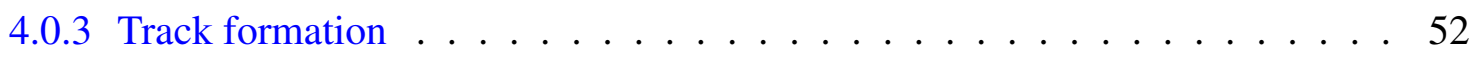

4.0 .4 Track Fitting . . . . . . . . . . . . . . . . . . . . . 54

4.0.4.1 Setting Track Properties $\ldots \ldots \ldots \ldots$

4.0.5 Cluster and Shower Formation $\ldots \ldots \ldots$. . . . . . . 57

4.0 .6 Event Formation . . . . . . . . . . . . . . . . . . 57

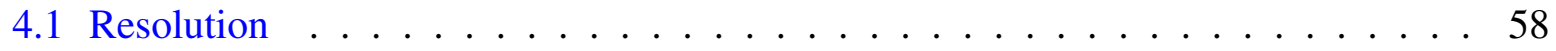

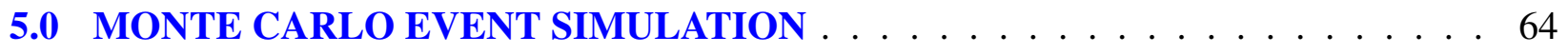

5.1 Quasi Elastic . . . . . . . . . . . . . . . . . . 65

5.2 Resonance . . . . . . . . . . . . . . . . 66

5.3 Deep Inelastic Scattering . . . . . . . . . . . . . . . . . . 68

5.4 RES-DIS overlap region . . . . . . . . . . . . . . . . . . . . . 69

5.5 Radiative Effects . . . . . . . . . . . . . . . . . . . . . . . . 74

5.6 Intra Nuclear Rescattering . . . . . . . . . . . . . . . . . . . . 74

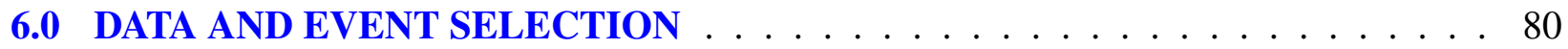

6.1 Event Selection . . . . . . . . . . . . . . . . . 80

6.2 Event subsamples . . . . . . . . . . . . . . . . . 82

6.3 Effect of cuts . . . . . . . . . . . . . . . . . 91

6.4 Acceptance Correction . . . . . . . . . . . . . . . . . . . 9 91

6.5 Data and MC simulation comparison . . . . . . . . . . . . . . . 101

6.6 Reverse field data . . . . . . . . . . . . . . . . . . . . 102

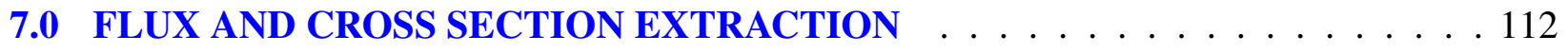


7.1 Overview . . . . . . . . . . . . . . . . . . 112

7.2 Flux extraction (low $v$ method) $\ldots \ldots \ldots \ldots \ldots \ldots \ldots \ldots$

7.3 Flux Measurement . . . . . . . . . . . . . . . . . 117

7.4 Cross section measurement . . . . . . . . . . . . . . . . . 130

7.5 Systematic uncertainties . . . . . . . . . . . . . . . . 134

7.5 .1 Energy scale . . . . . . . . . . . . . . . . . . . . . . . . 142

7.5.2 Cross section model . . . . . . . . . . . . . . . . . 143

7.5.3 Acceptance correction . . . . . . . . . . . . . . . . . . 143

7.5 .4 Background . . . . . . . . . . . . . . . . 146

7.5.5 Fiducial Volume . . . . . . . . . . . . . . . . . . . . 150

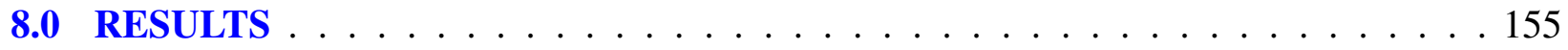

$8.1 v_{\mu} N$ and $\bar{v}_{\mu} N$ CC inclusive cross section $\ldots \ldots \ldots \ldots \ldots \ldots \ldots \ldots \ldots \ldots$

8.2 Ratio of $\bar{v}_{\mu} N$ CC to $v_{\mu} N$ CC inclusive cross section . . . . . . . . . 159

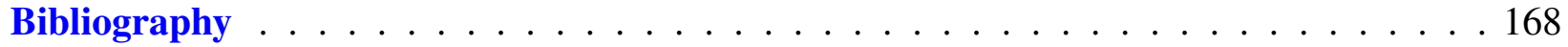

APPENDIX A. KALMAN FILTER . . . . . . . . . . . . . . . . . . . . 172

APPENDIX B. CORRECTIONS TO DIS CROSS SECTION MODEL . . . . . . . . . . 174

APPENDIX C. COMPARISON OF FLUX TUNING TECHNIQUES . . . . . . . . . 178

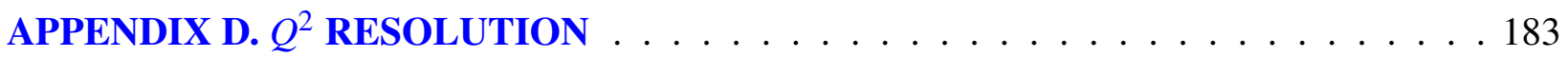

APPENDIX E. MINOS COLLABORATION . . . . . . . . . . . . . . . . . . 186 


\section{LIST OF TABLES}

1.1 Quarks and Leptons in the Standard Model. . . . . . . . . . . . . . . . . . . 4

1.2 Fundamental interactions and their relative strengths. . . . . . . . . . . 4

2.1 Comparison of measured and predicted magnetic field value. The first column shows the two positions("1" and "6") and the second column shows the number of planes for which the field was measured. The third and fourth columns show the measured and predicted average magnetic field in the area over which the induction coil was wound. The fifth column shows the ratio of measured to predicted field. . . 31

5.1 Parameters for the QEL model used in NEUGEN. The right hand column shows the errors on these parameters. The parameters have been taken from Ref.[8]. . . . 66

5.2 Parameters for the resonance model used in NEUGEN. The right hand column shows the errors on these parameters. The parameters have been taken from [45]. . 67

5.3 Parameters for the DIS model used in NEUGEN. The right hand column shows the errors on these parameters. The parameters have been taken from Ref.[5]. . . . . . 69

6.1 Number of events in the CC selected neutrino sample for each of the categories is shown in the first row. The second row shows the percentage contribution that each sample makes to the total sample. The third and fourth rows show the percentage $\mathrm{NC}$ and wrong sign contamination in each sample. . . . . . . . . . . . . 87

6.2 Number of events in the $\mathrm{CC}$ selected antineutrino sample for each of the categories is shown in the first row. The second row shows the percentage contribution that each sample makes to the total sample. The third and fourth rows show the percentage $\mathrm{NC}$ and wrong sign contamination in each sample. . . . . . . . . 88 
6.3 Effect of the selection criteria on the negatively(left) and positively(right) charged reconstructed samples. The numbers in bracket show the percentage of events lost due to the cut in the previous row. . . . . . . . . . . . . . . 96

7.1 Raw number of events in neutrino energy bins for the neutrino and antineutrino flux sample. . . . . . . . . . . . . . . . . . . . . . 120

7.2 Absolute neutrino(second column) and antineutrino(fourth column) flux as a function of energy. The third and fifth columns show the error(systematic uncertainty and statistical uncertainty added in quadrature) on the extracted flux. . . . . . . . 129

7.3 Raw number of events in energy bins for the neutrino and antineutrino cross section sample and the acceptance correction applied to each sample. The second(neutrino) and fourth(antineutrino) columns show the number of selected events and the third(neutrino) and fifth(antineutrino) columns show the acceptance correction. . . . . . . . . . 131

7.4 Summary of neutrino cross section results. The second column shows the energy bin average. The fourth, fifth and sixth columns show the statistical, systematic and normalization uncertainties respectively. The last column shows the total error, which is obtained by adding these three uncertainties in quadrature. . . . . . . . 138

7.5 Summary of antineutrino cross section results. The second column shows the energy bin average. The fourth, fifth and sixth columns show the statistical, systematic and normalization uncertainties respectively. The last column shows the total error, which is obtained by adding these three uncertainties in quadrature. . . . . . . . . 140

8.1 Average $Q^{2}$ for a given energy value calculated from MINOS generated sample. . . 156 


\section{LIST OF FIGURES}

1.1 Tree level Feynman diagram of a $v_{\mu} N \mathrm{CC}$ interaction. A neutrino interacts with a nucleon via the exchange of $\mathrm{W}$ boson to produce a muon and hadronic shower. . . . 7

1.2 LO diagrams that are included in QCD calculations. . . . . . . . . . . . 15

1.3 Feynman diagram for multiparton scattering. . . . . . . . . . . . . 16

2.1 Layout of the Neutrino beamline. Protons from the Main Injector hit a graphite target producing charged mesons. The charged mesons are focused by a pair of magnetic horns and subsequently decay to produce muons and muon neutrinos. The muons and uninteracted hadrons are absorbed and the neutrinos continue to the MINOS Near Detector $[61] . \quad \ldots \ldots \ldots$

2.2 Number of events in the near detector as a function of time. The five batch structure in a $10 \mu \mathrm{s}$ spill can be clearly seen. . . . . . . . . . . . . . . . . . . 18

2.3 Simulated flux spectrum of the low energy $v_{\mu}$ (a) and $\bar{v}_{\mu}$ beam(b) (which is $5.8 \%$ of the neutrino beam) in units of number of events $/ \mathrm{GeV} / \mathrm{m}^{2} / 10^{6}$ PoT. The inset figure shows the $v_{\mu}$ spectrum above $20 \mathrm{GeV}$. The PoT in the label stands for protons on

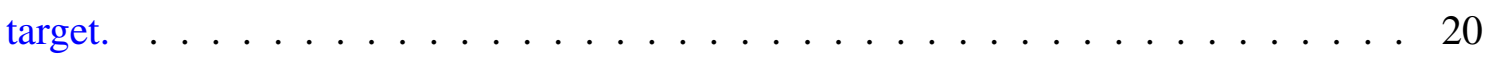

2.4 Transverse view of a Near Detector plane where the shaded region shows an instrumented active scintillator plane. . . . . . . . . . . . . . . . . 21

2.5 Longitudinal view of the near detector showing the different segmentations. . . . . 21

2.6 The U-V co-ordinate system used at the near detector. The coil hole is at the center of the co-ordinate system. . . . . . . . . . . . . . . . . . 22 
2.7 A partially instrumented plane is shown on the top and a fully instrumented plane on the bottom. The u-view is shown on the left and the v-view is shown on the right. Each partially(fully) instrumented plane is divided into three(five) scintillator modules, namely G,H,I (J,K,L,M,N) . . . . . . . . . . . . . . . . . . 23

2.8 Detailed arrangement of the strips in a typical near detector $\mathrm{U}$ and $\mathrm{V}$ plane for a partially and a fully instrumented plane. . . . . . . . . . . . . . . . . 23

2.9 A scintillator strip used in the near detector(a) and schematic of the optical connections in a scintillator module(b) . . . . . . . . . . . . . . . . 26

2.10 Face of M-64 phototube. Each of the 64 pixels $(2 \mathrm{~mm} \times 2 \mathrm{~mm})$ receive the output of a single scintillator strip. (a) Schematic of a PMT (b) . . . . . . . . . . . . . . 27

2.11 The setup used for measuring the B-H curve on a batch of steel that is used in the Near Detector. . . . . . . . . . . . . . . . . . . 28

2.12 The vendor provided B-H curve for the Near Detector. . . . . . . . . . . . . . . 29

2.13 The Near Detector field map estimated from the FEA program(a). The direction of the azimuthal magnetic field is shown by arrows. Change in the magnitude of the magnetic field as a function of $\mathrm{x}(\mathrm{m})$ at a constant $\mathrm{y}$ of $-10 \mathrm{~cm}(\mathrm{~b}) \ldots \ldots \ldots$. . . . .

2.14 The positions at which induction coils are wound to do a direct measurement of the magnetic field at the Near Detector. . . . . . . . . . . . . . . . . 31

2.15 Block diagram of a QIE chip. It has three main components, current splitter, integrator and a range selector. The chip digitizes the analogue signal from the PMTs. . 33

2.16 A MENU Board(a) and a MINDER Board(b) . . . . . . . . . . . . . . . . . 34

2.17 Schematic diagram of the MINOS Detector DAQ layout[43]. . . . . . . . . . . 35

2.18 Sketch of the Far Detector located at Soudan Mine, Minnesota. Each plane has full coverage of scintillators. The coil hole is located at the center of the detector and is the origin of the co-ordinate system. . . . . . . . . . . . . . . 37

3.1 Measured fractional composition of the T7 beamline in positive and negative polarities[43]. 40

3.2 The MINOS data calibration chain. . . . . . . . . . . . . . . . . 41

3.3 Detector response to hadrons and electron CALDET beams[43]. The unit MIP(minimum ionizing particle) is equivalent to MEU. The straight line shown in the plots is a fit to the high energy data. . . . . . . . . . . . . . . . . . 44 
$3.4 \pi^{+}$calorimeter signal distribution(MIPs) at momentum settings of $1 \mathrm{GeV} / \mathrm{c}, 4$ $\mathrm{GeV} / \mathrm{c}$ and $8 \mathrm{GeV} / \mathrm{c}$. Data is shown with the shaded histogram, GCALOR simulation is shown in blue and SLAC-GHEISHA simulation is shown in red. The histograms are normalized to unit area. . . . . . . . . . . . . . . . . . . 46

3.5 Proton calorimeter signal distribution(MIPs) at momentum settings of $1 \mathrm{GeV} / \mathrm{c}, 4$ $\mathrm{GeV} / \mathrm{c}$ and $8 \mathrm{GeV} / \mathrm{c}$. Data is shown with the shaded histogram, GCALOR simulation is shown in blue and SLAC-GHEISHA simulation is shown in red. The histograms are normalized to unit area. . . . . . . . . . . . . . . . . . . . 47

4.1 Muon neutrino charged current interaction simulated in the MINOS detector. The U versus $\mathrm{Z}$ and the $\mathrm{V}$ versus $\mathrm{Z}$ view are shown at the top and the bottom respectively. The colored boxes show the energy deposited in each scintillator strip. The last plot shows the pulse height distribution in each strip as a function of longitudinal distance. The energy deposited towards the beginning of the track is the largest and gradually decreases with distance. . . . . . . . . . . . . . . . . . . . 51

4.2 Standard reconstruction chain in MINOS. . . . . . . . . . . . . . . . . . 52

4.3 Figure on the left shows the hits in a Near Detector spill in the $\mathrm{U}$ versus $\mathrm{Z}$ view in the top and $\mathrm{V}$ versus $\mathrm{Z}$ view in the bottom. A spill with relatively few events has been chosen intentionally for clarity. Figure on the right shows the strips separated into slices based on timing and topology of hits. The Y-axis shows the number of

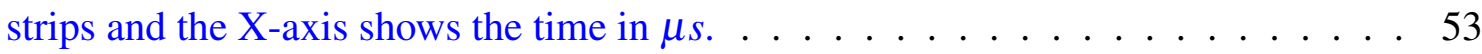

4.4 The various ways in which a triplet can be formed at the Near Detector[48]. The clusters must be in the same view. The blue circles represent a cluster and can contain more than one hit. . . . . . . . . . . . . . . . . . . . . . . 55

4.5 Resolution of momentum measured from range for tracks that stop in the detector with true muon energy between 3 and $4 \mathrm{GeV}$. The red line shows a Gaussian fit that has been performed between the two points at half maximum. The log version of the same plot is shown on the right. . . . . . . . . . . . . . . . 59 
4.6 Resolution of reciprocal momentum measured from curvature for events having true energy between 12 and $13 \mathrm{GeV}$. The red line shows a Gaussian fit that has been performed between the two points at half maximum. The log version of the same plot is shown on the right. . . . . . . . . . . . . . . . . 60

4.7 Muon angle resolution as a function of true muon angle. The resolution is plotted by fitting a gaussian to the distribution of (true $\theta_{\mu}$-reconstructed $\theta_{\mu}$ ) in each true

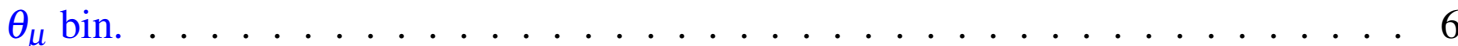

4.8 Hadronic and electromagnetic shower energy resolution as a function of available

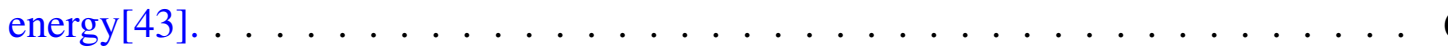

5.1 Effect of shifting the DIS parameter $A$ by $25 \%, B$ by $25 \%, C_{v 1 u}$ by $30 \%$ and $C_{v 2 u}$ by $40 \%$ on the charged lepton nucleon structure function $F_{2}$. The data points are from charged lepton scattering experiments. Each plot shows the $F_{2}$ versus $Q^{2}$ distribution for a given $x$ value. . . . . . . . . . . . . . . . 70

5.2 Neutrino(a) and antineutrino(b) cross section as a function of energy calculated from the model. The total cross section is shown in black, QEL in red, RES in green and DIS in blue. The dashed bands show the effect of modifying the model

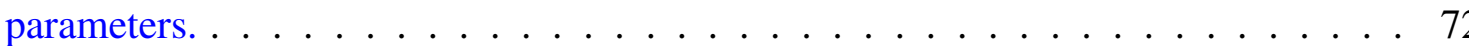

5.3 Ratio of antineutrino to neutrino cross section as a function of energy calculated from the model. The total cross section ratio is shown in black, QEL ratio in red, RES ratio in green and DIS ratio in blue. The dashed line at 0.5 shows the world average ratio between $30-200 \mathrm{GeV} \ldots \ldots \ldots \ldots$. . . . . . . . . . . . . . . .

5.4 QED loop corrections applied to charged current neutrino interactions. . . . . . . 74

5.5 Radiative corrections for charged current neutrino(black) and antineutrino(blue) differential cross section for incident energy of $6 \mathrm{GeV}$ as a function of $y$ for various

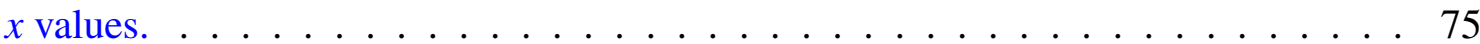

5.6 Ratio of radiative effect corrected total cross section to the Born level total cross section as a function of neutrino energy. . . . . . . . . . . . . . . 76 
5.7 A neutrino induced hadronic shower particle is created at an angle $\theta$ with momentum $\mathbf{p}$. The blue arrow joins the center of the nucleus to the interaction vertex $\mathbf{a}$ and the black arrow shows the shower particle direction. $\mathrm{X}$ is the distance traveled by the hadronic particle before it emerges from the nucleus [44] . . . . . . . . . 78

5.8 Interaction cross section for pion(a) and proton(b) as a function of kinetic energy from experimental data $[13,10]$. The total cross section is shown by the black line and the different contributions are shown in color. . . . . . . . . . . . . . 79

6.1 Number of PoTs as a function of time over a period of two years. . . . . . . . . . 81

6.2 Fiducial volume in the transverse view(a) and the longitudinal view(b) is shown by the blue shaded region. The solid black line shows the outline of the instrumented region and the dashed line shows the end of the calorimeter region. . . . . . . . . 83

6.3 Effect of muon energy selection criterion on fractional NC contamination for neutrino CC sample as a function of energy. . . . . . . . . . . . . . . . . 84

6.4 Muon momentum resolution as a function of radial distance from the coil hole. Three cases are shown, tracks that have only one strip(black line), tracks that have $2 \%$ of their strips(red line) and tracks that have $5 \%$ of their strips(blue) within the radial distance shown in the $\mathrm{x}$-axis. . . . . . . . . . . . . . . . . 85

6.5 $\mathrm{Z}$ vertex resolution as a function of the difference between beginning plane in the $\mathrm{u}$ and $\mathrm{v}$ view(a). Distribution of the $\mathrm{z}$ vertex resolution(b) with the standard CC

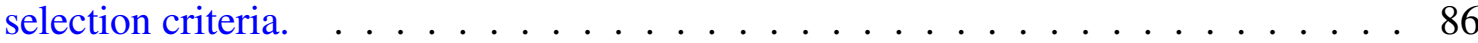

6.6 Energy distribution for neutrino and antineutrino CC subsamples. . . . . . . . . . . 89

6.7 Inelasticity distribution for neutrino and antineutrino CC subsamples. . . . . . . . 90

6.8 An illustration defining the Relative Angle variable[52]. Fig.(a) shows a straight line $\left(\overrightarrow{n_{V}}\right)$ along the initial direction of the track that projects the track vertex $\mathrm{V}$ to the point $\mathrm{P}$ on the last hit plane. $\mathrm{E}$ is the position of the actual track end. Fig.(b) shows the same track in the $\mathrm{x}-\mathrm{y}$ plane where the points V,P and $\mathrm{E}$ are shown relative to each other. For each track, a local Cartesian co-ordinate is constructed that has the line joining the coil hole $(\mathrm{C})$ and the point $\mathrm{V}$ as its $\mathrm{x}$-axis and a line orthogonal to it

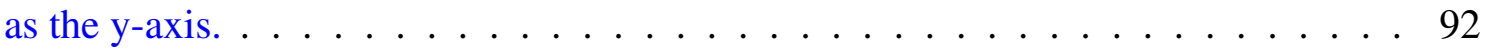


6.9 Distribution of Irelative angle- $\pi$ l in the reconstructed antineutrino sample(a). The black line shows the distribution for true neutrinos and the red line shows the same for true antineutrinos. Plot on the bottom(b) shows the efficiency, purity and product of efficiency and purity as a function of Irelative angle- $\pi$ l. . . . . . . . . . . 93

6.10 Fractional $\mathrm{CC}$ contamination for the reconstructed antineutrino sample as a function of the difference in the number of hit planes in the $U$ and $V$ view. . . . . . . . . 94

6.11 Efficiency of selection cuts for neutrino(a) and antineutrino(b) CC cross section sample. Each line shows the effect of the cuts in a cumulative manner. . . . . . . . 95

6.12 Acceptance correction from MC simulation for neutrino(a) and antineutrino(b) $\mathrm{CC}$ selected sample. . . . . . . . . . . . . . . . . . . . . . 97

6.13 Reciprocal of acceptance correction for the selected charged current neutrino sample and the sub-samples(shown by the colored lines). . . . . . . . . . . . . . . 98

6.14 Track end $\mathrm{y}$ versus end $\mathrm{x}$ for reconstructed neutrino sample that pass track fitting(top) and that fail track fitting(b) . . . . . . . . . . . . . . . 99

6.15 Effect of track fitter failure on total CC selected sample(both neutrino and antineutrino) as a function of track end $\mathrm{z}(\mathrm{m})$. The top plot(a) shows data and MC simulation comparison and the bottom plot(b) shows the MC event sample broken down into true neutrinos and true antineutrinos. . . . . . . . . . . . . . 100

6.16 Comparison of data and MC simulation distribution for track beginning $\mathrm{x}, \mathrm{y}$ and $\mathrm{z}$ for neutrinos(a) and antineutrinos(b) in the forward field mode. The points show data and red curve shows MC model after applying flux reweighting. The ratio of data to MC is shown below each distribution. The error bars show the statistical

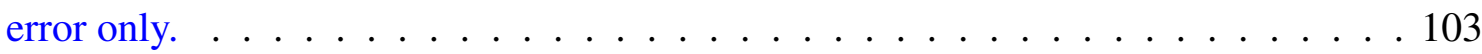

6.17 Comparison of data and MC simulation distribution for track end radial and track end $\mathrm{z}$ for neutrinos(a) and antineutrinos(b) in the forward field mode. The points show data and red curve shows MC model after applying flux reweighting. The ratio of data to $\mathrm{MC}$ is shown below each distribution. The error bars show the statistical error only. . . . . . . . . . . . . . . . . . . . . . 104 
6.18 Comparison of data and $\mathrm{MC}$ simulation distribution for the kinematic variables $E_{\mu}$, $E_{s h w}$ and $\theta_{\mu}$ for neutrinos(a) and antineutrinos(b) in the forward field mode. The points show data, the blue curve shows nominal MC model and the red curve shows MC model after applying flux reweighting. The ratio of data to $\mathrm{MC}$ is shown below each distribution. The error bars show the statistical and systematic uncertainties added in quadrature . . . . . . . . . . . . . . . . . . . . 105

6.19 Comparison of data and MC simulation distribution of inelasticity for neutrinos(a) and antineutrinos(b) in the forward field mode. The points show data, the blue curve shows nominal MC model and the red curve shows MC model after applying flux reweighting. The ratio of data to $\mathrm{MC}$ is shown below each distribution. The error bars show the statistical and systematic uncertainties added in quadrature. . . . 106

6.20 Comparison of reciprocal of acceptance correction for the reversed field and forward field neutrino(a) and antineutrino(b) sample. . . . . . . . . . . . . . . 108

6.21 Comparison of data and MC simulation distribution for track beginning $\mathrm{x}, \mathrm{y}$ and $\mathrm{z}$ for neutrinos(a) and antineutrinos(b) in the reverse field mode. The points show data and red curve shows MC model after applying flux reweighting. The ratio of data to MC is shown below each distribution. The error bars show the statistical error only. . . . . . . . . . . . . . . . . . . . . 109

6.22 Comparison of data and MC simulation distribution for track end radial and track end $\mathrm{z}$ for neutrinos(a) and antineutrinos(b) in the reverse field mode. The points show data and red curve shows MC model after applying flux reweighting. The ratio of data to $\mathrm{MC}$ is shown below each distribution. The error bars show the statistical error only. . . . . . . . . . . . . . . . . . . . 110

6.23 Comparison of data and MC simulation distribution for the kinematic variables $E_{\mu}$, $E_{s h w}$ and $\theta_{\mu}$ for neutrinos(a) and antineutrinos(b) in the reverse field mode. The points show data, the blue curve shows nominal MC model and the red curve shows MC model after applying flux reweighting. The ratio of data to $\mathrm{MC}$ is shown below each distribution. The error bars show the statistical and systematic uncertainties added in quadrature. . . . . . . . . . . . . . . . . . . . 111 
7.1 Purity as a function of energy for neutrino(left) and antineutrino(right) $\mathrm{CC}$ selected sample. . . . . . . . . . . . . . . . . . . . 114

7.2 Flow diagram for extracting the flux and cross section. $\Phi_{0}$ and $\sigma_{0}$ are the predicted flux and cross section from the model, $\Phi_{N E W}$ and $\sigma_{N E W}$ are the extracted flux and cross section. . . . . . . . . . . . . . . . . 115

7.3 The correction applied to the flux sample for neutrino(a) and antineutrino(b). The black line shows the correction for $v<1 \mathrm{GeV}$ flux sample(3-50 GeV range for neutrinos, 5-50 GeV range for antineutrinos), red line shows the correction for $v<2 \mathrm{GeV}$ flux sample(9-50 GeV range) and blue line shows the correction for $v<5 \mathrm{GeV}$ flux sample(18-50 GeV range). . . . . . . . . . . . . . 118

7.4 Overlap of flux and cross section sample for neutrino and antineutrino. The black curve shows the overlap of the $v<1 \mathrm{GeV}$ sample, red curve shows the overlap of the $v<2 \mathrm{GeV}$ sample and blue curve shows the overlap of the $v<5 \mathrm{GeV}$ sample. . 119

7.5 Acceptance correction from MC simulation for neutrino(a) and antineutrino(b) flux selected sample. The dashed lines show the energy values $(9 \mathrm{GeV}$ and $18 \mathrm{GeV})$ at which the value of the $v_{0}$ cut is increased. . . . . . . . . . . . . 122

7.6 Fractional contamination calculated from the flux sample in the neutrino(a) and antineutrino(b) flux selected sample. The red curve shows the fractional wrong sign contamination and the blue curve shows the fractional NC contamination. . . 123

7.7 Isoscalar correction applied to the neutrino and antineutrino flux sample. The black line shows the correction for neutrino and the red line shows the correction for antineutrino. . . . . . . . . . . . . . . . . . . 124

7.8 Ratio of the extracted flux to simulated flux for neutrinos(a) and antineutrinos(b) as a function of neutrino energy. The shaded box shows the statistical and systematic uncertainties added in quadrature and the error bars show the statistical error only. . 125

7.9 Comparison of the fiducial volume used in this analysis with a circular area of radius $0.75 \mathrm{~m}$. The circular area has been used to smear the MC flux that is calculated at the center of the detector. . . . . . . . . . . . . . . 126

7.10 Ratio of smeared MC flux over a circular area of radius $0.75 \mathrm{~m}$ to the flux at the center of the detector. . . . . . . . . . . . . . . . . 127 
7.11 Extracted flux for neutrinos(a) and antineutrinos(b) as a function of neutrino energy. The error bars(in black) show the statistical error. The shaded boxes show the statistical and systematic uncertainties added in quadrature. . . . . . . . . . . . 128

7.12 Effect of modifying the $v<1 \mathrm{GeV}$ cut to $v<0.75 \mathrm{GeV}$ on the measured cross

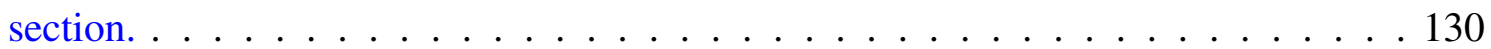

7.13 Isoscalar correction applied to the neutrino and antineutrino cross section sample. The black line shows the correction for neutrino and the red line shows the correction for antineutrino. . . . . . . . . . . . . . . . 133

7.14 Contamination from NC and wrong-sign events as a function of energy in the neutrino(a) and antineutrino(b) selected charged current sample as calculated from the MC event sample. The error bars show the statistical error only. . . . . . . . . . . 135

7.15 Contamination from NC and wrong-sign events as a function of inelasticity in the neutrino(a) and antineutrino(b) selected charged current sample as calculated from the MC event sample. The error bars show the statistical error only. . . . . . . . . 136

7.16 Neutrino charged-current inclusive cross section as a function of energy for an isoscalar iron target. The black error bars show the statistical uncertainty and the shaded boxes show the statistical and systematic uncertainties added in quadrature. The dotted red band shows the normalization error that is about $1.5 \%$. The solid black line shows the world cross section value $\left(0.675 \times 10^{-38} \mathrm{~cm}^{2} / \mathrm{GeV}\right)$ in the 30 $50 \mathrm{GeV}$ range and the dashed black line shows this value extrapolated to lower energies. The neutrino cross section is normalized to this value. . . . . . . . . 137

7.17 Antineutrino charged-current inclusive cross section as a function of energy for an isoscalar iron target. The black error bars show the statistical uncertainty and the shaded boxes show the statistical and systematic uncertainties added in quadrature. The dotted red band shows the error on MINOS normalization constant and the error on world cross section data added in quadrature(about $1.5 \%$ ). The solid black line shows the world cross section value $\left(0.329 \times 10^{-38} \mathrm{~cm}^{2} / \mathrm{GeV}\right)$ in the $30-50 \mathrm{GeV}$ range and the dashed black line shows this value extrapolated to lower energies. . . 139 
7.18 Ratio of antineutrino-nucleon to neutrino-nucleon cross section as a function of energy. The black error bars show the statistical uncertainty and the shaded boxes show the statistical and systematic uncertainties added in quadrature. The solid black line shows the value of this ratio from other experiments between 30-200 $\mathrm{GeV}$ at $0.504 \pm 0.003[3] . \ldots \ldots \ldots$. . . . . . . . . . . . . . . . . . . . . . . . .

7.19 Effect of the energy scale uncertainty on the neutrino(a) and antineutrino(b) extracted flux. The black line shows the effect of shifting the muon energy scale by $2 \%$ for stopping muons and $4 \%$ for exiting muons. The red line shows the effect of shifting the hadronic energy scale by $5.6 \%$ and the blue line shows the uncertainty from final state interactions. . . . . . . . . . . . . . . . . . . 144

7.20 Effect of the energy scale uncertainty on the neutrino(a) and antineutrino(b) extracted cross section. The black line shows the effect of shifting the muon energy scale by $2 \%$ for stopping muons and $4 \%$ for exiting muons. The red line shows the effect of shifting the hadronic energy scale by $5.6 \%$ and the blue line shows the uncertainty from final state interactions. . . . . . . . . . . . . . . . 145

7.21 Uncertainty in the intranuclear rescattering model as a function of true shower energy.146

7.22 Ratio of reverse field to forward field extracted flux for neutrinos(left) and antineutrinos(right) . . . . . . . . . . . . . . . . . . 147

7.23 Ratio of reverse field to forward field extracted $\sigma / E$ for neutrinos(left) and antineutrinos(right) . . . . . . . . . . . . . . . . . 147

7.24 Effect of modifying the minimum $E_{\mu}$ cut from its standard value of $1.5 \mathrm{GeV}$ to 2 $\mathrm{GeV}$ and $1 \mathrm{GeV}$ on the measured cross section. . . . . . . . . . . . . . 148

7.25 Data and MC comparison of MC comparison of the antineutrino CC sample as a function of energy. The top plot shows the distribution and the bottom plot shows the ratio of data to MC. Two cases are shown, one in which the standard MC contamination is subtracted from the data, the other in which double the MC contamination is subtracted. . . . . . . . . . . . . . . . . . . . . 149

7.26 Systematic uncertainty from wrong sign contamination in the antineutrino extracted cross section. . . . . . . . . . . . . . . . . . 150 
7.27 Distribution of majC for the total reconstructed antineutrino sample. The distribution for the wrong sign contamination is shown in red. $\ldots \ldots \ldots \ldots 151$

7.28 Fractional wrong sign contamination in the $\mathrm{CC}$ antineutrino sample by applying the relative angle(red) and majority curvature(black) selection criteria. . . . . . . . . . 151

7.29 Fiducial volume divided into left and right sections in the transverse view using $\mathrm{x}$ vertex $>1.5 \mathrm{~m}(<1.5 \mathrm{~m})$ (a). Comparison of the measured $\sigma / E$ for neutrino and antineutrino between the two sections (b). . . . . . . . . . . . . . 153

7.30 Fiducial volume divided into up and down sections in the transverse view using y vertex $>0 \mathrm{~m}(<0 \mathrm{~m})$ (a). Comparison of the measured $\sigma / E$ for neutrino and antineutrino between the two sections (b) . . . . . . . . . . . . . . . . . 154

8.1 MINOS neutrino and antineutrino charged-current inclusive cross section compared with other experimental[18, 31, 26, 21, 12, 16, 30, 25, 28, 14, 23, 46, 17, 55, $20,11,27]$ results. The error bars show the statistical, systematic and normalization uncertainties added in quadrature. The solid black line shows the average world cross section in the $30-50 \mathrm{GeV}$ region for the neutrino $\left(0.675 \times 10^{-38} \mathrm{~cm}^{2} / \mathrm{GeV}\right)$ and the antineutrino $\left(0.329 \times 10^{-38} \mathrm{~cm}^{2} / \mathrm{GeV}\right)$ cross section respectively. The dashed line shows these high energy values extrapolated to lower energies. . . . . . . . . 160

8.2 Helicity of neutrinos(antineutrinos) and quarks in a weak interaction. The blue arrows show the direction of the spin and the black arrows show the direction of the momentum. . . . . . . . . . . . . . . . . . . . . . . 161

8.3 Energy dependence of the terms $\left(P+\frac{1}{3} \bar{P}\right)$ and $\left(\bar{P}+\frac{1}{3} P\right)$. The black line shows the value of this quantity when a $\mathrm{W}$ cut of $1 \mathrm{GeV}$ is applied and the red line shows the quantity for a W cut of $2 \mathrm{GeV}$. . . . . . . . . . . . . . . . . . . . . . . . . . 161

8.4 Energy dependence of the terms $P$ and $\bar{P}$. The black line shows the value of this quantity when a $\mathrm{W}$ cut of $1 \mathrm{GeV}$ is applied and the red line shows the quantity for a W cut of $2 \mathrm{GeV} . \ldots \ldots \ldots$

8.5 MINOS measured cross section compared to the cross section predicted by the NEUGEN cross section model for neutrinos(a) and antineutrinos(b). . . . . . . . . 163 
8.6 Ratio of antineutrino-nucleon to neutrino-nucleon cross section as a function of energy. The black error bars show the statistical uncertainty and the shaded boxes show the statistical and systematic uncertainty added in quadrature. The solid blue line shows the value of this ratio from other experiments between $30-200 \mathrm{GeV}$ at $0.504 \pm 0.003$. Ratio of $P+\frac{1}{3} \bar{P}$ to $\bar{P}+\frac{1}{3} P$ is plotted as a function of energy. The black line shows this term when a $\mathrm{W}$ cut of $1 \mathrm{GeV}$ is applied and the red line shows this term when a $\mathrm{W}$ cut of $2 \mathrm{GeV}$ is applied. . . . . . . . . . . . . . 164

8.7 Ratio of $\bar{P} / P$ is plotted as a function of neutrino energy. The black line shows the value of this quantity when a $\mathrm{W}$ cut of $1 \mathrm{GeV}$ is used and the red line shows the value of this quantity when a $\mathrm{W}$ cut of $2 \mathrm{GeV}$ is used. . . . . . . . . . . 165

8.8 Ratio of antineutrino to neutrino cross section as a function of energy. The red line shows this ratio calculated from the cross section model. . . . . . . . . . . . 166

B1 Ratio of $\sigma(\bar{v}) / \sigma(v)$ from the NEUGEN cross section model. The red line shows the default ratio, the blue line shows the ratio after using $\xi_{W}$ in the cross section equation and the red line shows the ratio after applying a $v$ and $\mathrm{W}$ threshold. The dashed line shows the average value of this ratio between $30-200 \mathrm{GeV}$ from experiments. . . . . . . . . . . . . . . . . 176

B2 Comparison of neutrino low- $v$ correction calculated from the default cross section model(NEUGEN3) and the corrected model. . . . . . . . . . . . . . . 177

B3 Comparison of antineutrino low- $v$ correction calculated from the default cross section model(NEUGEN3) and the corrected model. . . . . . . . . . . . . . . . 177

C1 Comparison of flux tuning using the low- $v$ and SKZP method for the neutrino sample. The bottom plot shows the ratio of these two weights. The low- $v$ weights have statistical and systematic uncertainties added in quadrature, the SKZP weights have only the statistical uncertainties. . . . . . . . . . . . . . . . . 180

C2 Comparison of flux tuning using the low $-v$ and SKZP method for the antineutrino sample. The bottom plot shows the ratio of these two weights. The low- $v$ weights have statistical and systematic uncertainties added in quadrature, the SKZP weights have only the statistical uncertainties. . . . . . . . . . . . . . 181 
C3 Comparison of data and MC simulation. The bottom plot shows the ratio of these two weights. The low- $v$ weights have statistical and systematic uncertainties added in quadrature, the SKZP weights have only the statistical uncertainties. . . . . . . . 182

D1 Distribution of (reconstructed $Q^{2}$ - true $Q^{2}$ ) for a neutrino DIS selected sample in four true $Q^{2}$ bins. . . . . . . . . . . . . . . . . . . . 184

D2 Mean of true angle distribution as a function of true $Q^{2}$ for the neutrino enhanced DIS sample. . . . . . . . . . . . . . . . . . 185 
This thesis is dedicated to Suddhashil and my parents for their encouragement and support. 


\section{PREFACE}

The last six years have been an amazing journey which had a big impact on my professional as well as my personal life. I would have to begin by thanking my parents and my husband who have been a huge source of encouragement right from the beginning. Without their unconditional support, this work wouldn't have been possible.

My field of work gave me the opportunity to work with people from different countries and different backgrounds. Interacting with my colleagues from MINOS, especially from the Near Detector group has been an immensely beneficial experience. The countless emails I have exchanged with Hugh Gallagher, Tingjun Yang, Trish Vahle and Mike Kordosky, to name a few, have helped me understand the complicated codes that are used in the experiment.

I have been extremely fortunate to find an advisor like Donna Naples. She has given me invaluable advice on my research and always took care to explain the underlying physics behind my work and all the other projects in the experiment. For a major part of my graduate life I did not have a driver's license, she always made sure that I never got stranded without a car or be left without food. We have shared hotel rooms, car rides, plane rides (and even our birthday) and had long conversations on varied topics that included physics, movies, tra velling and cultural differences between the east and west(among many others). She indeed made my stay in Pittsburgh very special. 


\subsection{INTRODUCTION}

Neutrino scattering experiments have played an important role in measuring many standard model parameters and in investigating physics beyond the standard model. Neutrino and charged lepton deep inelastic scattering experiments provided the first definte proof of the quark sub-structure of nucleons. Modern experiments use intense neutrino beams and massive detectors to compensate for the small interaction probability of neutrinos.

The scattering cross section is a sum of three sub-processes, elastic, resonance and deep inelastic scattering interactions. In elastic interactions, that dominate at energies below $10 \mathrm{GeV}$, the resolution capacity of the neutrino probe is low and it scatters off entire nucleons. At intermediate energies, between 10 to $20 \mathrm{GeV}$, the nucleon gets excited into a resonant state which then decays to produce hadrons. As the energy increases, neutrinos undergo deep inelastic scattering in which they have enough energy to resolve the constituents of a nucleon (known as partons). In this region, the neutrino cross section becomes linearly dependent on neutrino energy in confirmation to the quark parton model.

Several experiments $[18,26,25,46,55,20]$ in the late 1980s and 1990s have measured the neutrino cross section above $30 \mathrm{GeV}$ with a precision of $2 \%$. Most experiments [31, 21, 12, 16, $28,17,11]$ conducted at lower energies $\left(E_{v}<30 \mathrm{GeV}\right)$ in the 1970 s and early 1980 s have a much larger uncertainty of the order of $10 \%$ on the extracted cross section. The main contribution to this

uncertainty came from flux measurements which used a simulation of the neutrino beam. Recently $\operatorname{NOMAD}([27])$ has measured the cross section in the energy range $2.5-300 \mathrm{GeV}$ with a precision of up to $4 \%$.

This thesis presents the measurement for energy dependence of neutrino and antineutrino inclusive charged current cross section on an isoscalar iron target from the high statistics data sample collected at the MINOS Near Detector. The neutrino measurement is between 3-50 GeV with an 
uncertainty (better than $5 \%$ above $4 \mathrm{GeV}$ ) that is smaller that most previous experiments. Although the NOMAD result has a comparable precision, it relies on a particle production model to predict the neutrino flux whereas we use a low hadronic energy sample to extract the flux from the MINOS data. There are very few existing measurements of the antineutrino cross section and the ratio of antineutrino to neutrino cross section in the low energy region. MINOS makes an important contribution in the 5-50 GeV range. The antineutrino cross section measurement has relatively large uncertainties compared to that of the neutrino, of the order of $10 \%$ in the low and high energy regions because of the smaller size of the sample. The MINOS measurement covers an interesting region that shows the transition from elastic scattering at lower energies to the deep inelastic scattering regime at higher energies where the scaling behavior becomes prominent.

The long baseline oscillation experiments search for oscillations in the few $\mathrm{GeV}$ region. The current and future experiments use two detectors that help in reducing the uncertainty in the measurement of the oscillation parameters. To perform this measurement, it is important to know the relation between the visible neutrino energy in the detector and the actual neutrino energy. This entails a detailed knowledge of the neutrino flux and cross section. The MINOS measurement will help in constraining the cross section and flux measurements in the low energy range.

Section 1.1 of the first chapter gives an introduction to the standard model in particle physics with special emphasis on neutrinos. Section 1.2 begins with a discussion of the kinematic variables and then describes the cross section formula for each of the sub-processes. The rest of the thesis is arranged as follows:

- Chapter 2 describes the neutrino beam and the MINOS detectors.

- Chapter 3 discusses the detector calibration.

- Chapter 4 discusses the reconstruction scheme.

- Chapter 5 provides a detailed description of the Monte Carlo simulation used in MINOS.

- Chapter 6 describes the data and event selection criteria.

- Chapter 7 discusses the flux and cross section measurements and evaluation of systematic uncertainties.

- Chapter 8 discusses and compares the MINOS results with other experiments. 


\subsection{STANDARD MODEL AND NEUTRINOS}

Standard Model of particle physics unifies three of the four known fundamental interactions, weak, electromagnetic and strong. The gravitational force is not a part of this unification but it is much weaker than the other three forces. In this model, the elementary particles are divided into two categories, fermions and bosons. The fermions are the building blocks and the bosons act as mediators of the fundamental interactions. All matter is made up of these elementary building blocks, for example an atom is made up of electrons and a nucleus that consists of protons and neutrons which are each composed of quarks.

The fermions can be sub-divided into two groups, leptons and quarks, depending on the type of interaction in which they participate. Quarks can undergo all types of interactions whereas leptons can undergo weak and electromagnetic (only the charged leptons) interactions. There are six leptons in all, three are neutral leptons called neutrinos (electron neutrino $v_{e}$, muon neutrino $v_{\mu}$ and tau neutrino $v_{\tau}$ ) and each one has a charged partner (electron $e^{-}$, muon $\mu^{-}$and tau $\tau^{-}$). The quarks have a fractional charge, with three quarks up(u), charm(c) and top(t) carrying a charge of $+\frac{2}{3}$ and the other three quarks down(d), strange(s) and bottom(b) carrying a charge of $-\frac{1}{3}$. Both quarks and leptons are categorized into three generations of "doublets" by arranging them in order of increasing mass. Table 1.1 shows the quarks, leptons and their electrical charge. All the quarks and leptons have a corresponding antiparticle that are also part of the Standard Model. The antiparticle of each fermion has the same mass but opposite charge(opposite spin in case of antineutrinos). The bosons that are part of the Standard Model are photons, $W^{ \pm}, Z^{0}$ and gluons that mediate the electromagnetic, weak and strong interactions respectively. Table 1.2 shows a list of all the mediators and the relative strength of the four interactions.

Neutrinos are assumed to be massless in the Standard Model because there was no evidence for neutrino mass when this model was built. It is now a well established fact that neutrinos can oscillate from one flavor to the other implying that they have a non-zero(albeit small) mass. The neutrino flavor eigenstates $v_{\alpha}\left(v_{e}, v_{\mu}\right.$ and $\left.v_{\tau}\right)$ and the mass eigenstates, $v_{i}$ are related by a unitary transformation matrix $U$, such that $v_{\alpha}=U v_{i}$. The matrix $U$, that is analogous to the CabibboKobayashi-Maskawa mixing matrix for quarks, can be written as 


\begin{tabular}{|c|c|c|c|c|}
\hline \multirow{2}{*}{$\begin{array}{c}\text { Type of } \\
\text { fermion }\end{array}$} & \multirow{2}{*}{$\begin{array}{c}\text { Electrical } \\
\text { Charge }\end{array}$} & \multicolumn{3}{|c|}{ Generation } \\
\cline { 3 - 5 } & I & II & III \\
\hline \hline QUARKS & $+\frac{2}{3}$ & $\mathrm{u}$ & $\mathrm{c}$ & $\mathrm{t}$ \\
& $-\frac{1}{3}$ & $\mathrm{~d}$ & $\mathrm{~s}$ & $\mathrm{~b}$ \\
\hline \hline LEPTONS & 0 & $v_{e}$ & $v_{\mu}$ & $v_{\tau}$ \\
& 1 & $e$ & $\mu$ & $\tau$ \\
\hline
\end{tabular}

Table 1.1: Quarks and Leptons in the Standard Model.

\begin{tabular}{|c|c|c|c|}
\hline Interaction & Participating particles & Mediating boson & Relative strengths \\
\hline \hline Strong & Quarks & gluon $(g)$ & $>1$ \\
\hline Electromagnetic & Quarks and charged leptons & photon $(\gamma)$ & $\sim 1 / 137$ \\
\hline Weak & All & $W^{ \pm}, Z^{0}$ & $10^{-5}$ \\
\hline Gravitational & All & Graviton & $10^{-42}$ \\
\hline
\end{tabular}

Table 1.2: Fundamental interactions and their relative strengths. 


$$
\left(\begin{array}{ccc}
U_{e 1} & U_{e 2} & U_{e 3} \\
U_{\mu 1} & U_{\mu 2} & U_{\mu 3} \\
U_{\tau 1} & U_{\tau 2} & U_{\tau 3}
\end{array}\right)=\left(\begin{array}{ccc}
1 & 0 & 0 \\
0 & c_{23} & s_{23} \\
0 & -s_{23} & c_{23}
\end{array}\right) \times\left(\begin{array}{ccc}
c_{13} & 0 & s_{13} e^{-i \delta} \\
0 & 1 & 0 \\
-s_{13} e^{i \delta} & 0 & c_{13}
\end{array}\right) \times\left(\begin{array}{ccc}
c_{12} & s_{12} & 0 \\
-s_{12} & c_{12} & 0 \\
0 & 0 & 1
\end{array}\right),
$$

where, $c_{i j}=\cos \theta_{i j}, s_{i j}=\sin \theta_{i j}$ and $\delta$ is a phase factor which is non zero only if neutrino mixing violates CP symmetry.

The transition probability from one neutrino flavor $\alpha$ to another flavor $\beta$ can be written as

$$
P(\alpha \rightarrow \beta)=\left|\sum_{i} U_{\beta i} e^{-i\left(E_{i} t-p_{i} x\right)} U_{\alpha i}^{*}\right|^{2}
$$

The propagation of the mass eigenstates in space and time is given by the exponential factor. In the ultra relativistic limit, $\left|\overrightarrow{p_{i}}\right| \gg m_{i}$

$$
E_{i}=\sqrt{p_{i}^{2}+m_{i}^{2}} \simeq p_{i}+\frac{m_{i}^{2}}{2 p_{i}^{2}} \approx E_{i}+\frac{m_{i}^{2}}{2 E_{i}} .
$$

In this limit, Eq. 1.2 can be rewritten as

$$
P(\alpha \rightarrow \beta)=\sum_{i}\left|U_{\beta i}\right|^{2}\left|U_{\alpha i}\right|^{2}+R e \sum_{i \neq j} U_{\beta i} U_{\beta j}^{*} U_{\alpha i}^{*} U_{\alpha j} e^{-i \Delta m_{i j}^{2} L / 2 E}
$$

where, $\Delta m_{i j}^{2}=\left|m_{i}^{2}-m_{j}^{2}\right|, L$ is the length for which the neutrinos are allowed to oscillate.

There are six independent parameters (three angles, one phase factor, two independent $\Delta m^{2}$ ) that can be reduced by assuming $\Delta m_{32}^{2} \ll \Delta m_{21}^{2}$ (neutrino masses are strongly ordered). The simplified oscillation probability under this assumption can be written as

$$
\begin{aligned}
& P(\alpha \rightarrow \alpha)=1-4 U_{\alpha 3}^{2}\left(1-U_{\alpha 3}^{2}\right) \sin ^{2}\left(1.27 \Delta m_{32}^{2} L / E\right)=1-\sin ^{2} 2 \theta_{23} \sin ^{2}\left(1.27 \Delta m_{32}^{2} L / E\right) \\
& P(\alpha \rightarrow \beta)=4 U_{\alpha 3}^{2} U_{\beta 3}^{2} \sin ^{2}\left(1.27 \Delta m_{32}^{2} L / E\right)=4 \sin ^{2} 2 \theta_{23} \sin ^{2}\left(1.27 \Delta m_{32}^{2} L / E\right) .
\end{aligned}
$$

Massive neutrinos can be included in the Standard Model by using a modified Lagrangian. 


\subsection{NEUTRINO CHARGED CURRENT CROSS SECTION}

\subsubsection{Kinematic Quantities}

The neutrino-nucleon interaction are of two types: Charged Current(CC) in which a charged $W^{+(-)}$ boson is exchanged and Neutral Current in which a neutral $Z^{0}$ boson is exchanged. The physics of a CC interaction is presented here since the analysis described in this thesis involves studying such interactions. Fig. 1.1 shows the tree level Feynman diagram of a neutrino-nucleon CC interaction. The Lorentz invariant quantities of this interaction are,

- energy transfer to the hadronic system

$$
v=\frac{P \cdot q}{M}
$$

- inelasticity

$$
y=\frac{P \cdot q}{P \cdot k_{1}},
$$

- negative squared four-momentum of the boson

$$
Q^{2}=-q^{2}=-\left(k_{1}-k_{2}\right)^{2}
$$

- Bjorken scaling variable

$$
x=\frac{Q^{2}}{2 P \cdot q},
$$

- square of the center of mass(c.o.m.) energy of the boson-nucleon system

$$
W^{2}=(P+q)^{2}
$$

- and square of the center of mass(c.o.m.) energy of the neutrino-nucleon system

$$
s=\left(P+k_{1}\right)^{2} .
$$

In these equations $k_{1}, k_{2}, q$ and $P$ are the four-momentum of the neutrino, muon, $W$ boson and the nucleon respectively and $M$ is the nucleon mass.

In the laboratory Frame, the nucleon is assumed to be at rest and the neutrino initially travels in the $\mathrm{z}$-direction. The four-momentum of the particles in this frame is 


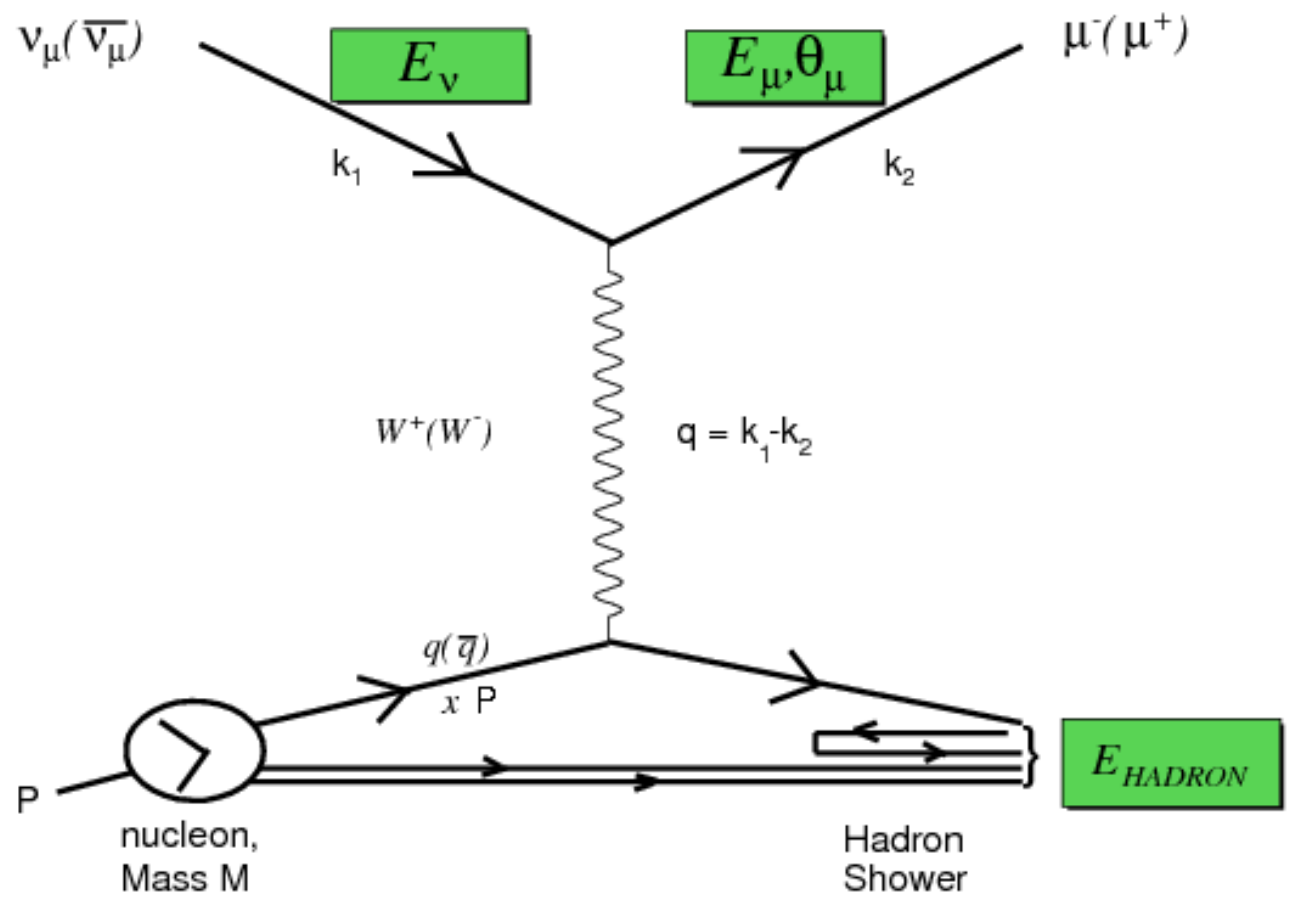

Figure 1.1: Tree level Feynman diagram of a $v_{\mu} N$ CC interaction. A neutrino interacts with a nucleon via the exchange of $\mathrm{W}$ boson to produce a muon and hadronic shower. 
1. $P=(M, 0,0,0)$,

2. $k_{1}=\left(E_{v}, 0,0, E_{v}\right)$,

3. $k_{2}=\left(E_{\mu}, 0, k_{2} \sin \theta_{\mu}, k_{2} \cos \theta_{\mu}\right)$,

where, $E_{\mu}$ is the muon energy, $E_{\nu}$ is the neutrino energy and $\theta_{\mu}$ is the muon direction w.r.t. the neutrino.

The invariant quantities can be simplified in the laboratory frame (neglecting muon mass) as follows:

- $v=E_{H A D}\left(E_{H A D}\right.$ is the hadronic shower energy).

- $y=\frac{E_{H A D}}{E_{v}}$.

- $Q^{2}=2 E_{v} E_{\mu}\left(1-\cos \theta_{\mu}\right)$.

- $x=\frac{Q^{2}}{2 M v}$.

- $W^{2}=M^{2}-Q^{2}+2 M v$.

- $s=M^{2}+2 M E_{v}$.

\subsubsection{Neutrino Interaction cross section}

Fermi's Golden rule gives the cross section of an interaction as

$$
d \sigma=d \Gamma \frac{|\mathscr{M}|^{2}}{\Phi}
$$

where, $\mathscr{M}$ is the invariant amplitude of the scattering, $d \Gamma$ is the phase space factor and $\Phi$ is the incident flux. The Born level invariant amplitude can be written as

$$
\mathscr{M}=\sqrt{2} G_{F} \bar{u}_{\mu} \gamma_{\alpha}\left(1-\gamma_{5}\right) u_{v} \frac{1}{1+Q^{2} / M_{W}^{2}}<X\left|J_{C C}\right| \mathscr{N} ; p, s>
$$

where, $G_{F}$ is the Fermi Coupling Constant, $M_{W}$ is the $W^{ \pm}$Boson mass, $\bar{u}_{\mu} \gamma_{\alpha}\left(1-\gamma_{5}\right) u_{v}$ is the weak leptonic current and $\left\langle X\left|J_{C C}\right| \mathscr{N} ; p, s>\right.$ is the hadronic current. The next three sub-sections discuss the neutrino cross section equation for each of the three regimes, quasi-elastic(QEL), resonance(RES) and deep inelastic scattering(DIS) interactions. 
1.2.2.1 Quasi Elastic Interactions In the case of QEL interactions, neutrino(antineutrino) scatters off a nucleon producing a muon and a proton(neutron)

$$
v\left(\bar{v}_{\mu}\right) n(p) \rightarrow \mu^{-}\left(\mu^{+}\right) p(n)
$$

The general QEL hadronic current can be expressed as [56]

$$
<P\left(p^{\prime}\right)\left|J_{C C}\right| \mathscr{N}(p)>=\bar{P}\left(p^{\prime}\right)\left[F_{V}^{1} \gamma_{\mu}+\frac{i \sigma_{\mu \nu} q^{v} \xi F_{V}^{2}}{2 M}+\frac{q_{\mu} F_{S}}{M}+F_{A} \gamma_{5} \gamma_{\mu}+\frac{F_{P} \gamma_{5} q_{\mu}}{M}+\frac{\gamma_{5}\left(p+p^{\prime}\right)_{\mu} F_{T}}{M}\right] \mathscr{N}(p)
$$

where, $F_{S}, F_{P}, F_{V}, F_{A}$ and $F_{T}$ are the scalar, pseudoscalar, vector, axial-vector and tensor form factors of the nucleon and $p^{\prime}$ is the four momentum of the outgoing nucleon.

The differential cross section in the Lab Frame can be written as

$$
\frac{d \sigma^{v(\bar{v})}}{d q^{2}}=\frac{M^{2} G_{F}^{2} \cos ^{2} \theta_{C}}{8 \pi E_{v}^{2}}\left[A\left(q^{2}\right) \mp B\left(q^{2}\right) \frac{(s-u)}{M^{2}}+C\left(q^{2}\right) \frac{(s-u)^{2}}{M^{4}}\right]
$$

where, $s, t=q^{2}$ and $u$ are the Mandelstam variables and $\theta_{C}$ is the Cabibbo mixing angle. The functions $A, B$ and $C$ are combinations of the form factors. Eq. 1.16 is the most general form of the QEL interaction.

1.2.2.2 Resonance Interactions Resonance interactions excite the nucleon $\left(v N \rightarrow v N^{*}\right)$ which then decays into low multiplicity final states:

$$
\begin{gathered}
v_{\mu} p(n) \rightarrow \mu^{-} \pi^{+} p(n), \quad \bar{v}_{\mu} n(p) \rightarrow \mu^{+} \pi^{-} n(p), \\
v_{\mu} n \rightarrow \mu^{-} \pi^{0} p, \quad \bar{v}_{\mu} p \rightarrow \mu^{+} \pi^{0} n .
\end{gathered}
$$

The hadronic current for this case can be written as $\left\langle N *\left|J_{\beta}^{+}(0)\right| N\right\rangle$, where the hadronic current operator is a combination of a vector $\left(F_{\beta}^{V}\right)$ and an axial $\left(F_{\beta}^{A}\right)$ part

$$
J_{\beta}=V_{\beta}-A_{\beta}=2 M_{R}\left(F_{\beta}^{V}-F_{\beta}^{A}\right),
$$

and $M_{R}$ is the resonance mass parameter. 
The leptonic current $\bar{u}_{\mu} \gamma_{\alpha}\left(1-\gamma_{5}\right) u_{v}$ can be expressed in terms of the polarization vectors of the intermediate boson. If $e_{L}^{\mu}, e_{R}^{\mu}$ and $e_{0}^{\mu}$ correspond to the left-handed, right handed and scalar polarization vectors and the three momentum of the virtual boson is along the $\mathrm{z}$ direction, then

$$
\begin{aligned}
q^{\alpha} & =(v, 0,0, Q), \\
e_{L}^{\alpha} & =\frac{1}{\sqrt{2}}(0,1,-i, 0), \\
e_{R}^{\alpha} & =\frac{1}{\sqrt{2}}(0,1,-i, 0), \quad \text { and } \\
e_{0}^{\alpha} & =(1,0,0,0) .
\end{aligned}
$$

The leptonic current can be re-written using Eq. 1.19 as

$$
\bar{u}_{\mu} \gamma^{\alpha}\left(1-\gamma_{5}\right) u_{v}=-2 \sqrt{2} E_{v} \sqrt{\frac{Q^{2}}{Q^{\prime 2}}}\left\{u \cdot e_{L}^{\alpha}-v \cdot e_{R}^{\alpha}+\sqrt{2 u v} \cdot e_{S}^{\alpha}\right\}
$$

where, $Q^{\prime 2}=v^{2}-Q^{2}, u=\frac{E_{v}+E_{\mu}+Q^{\prime}}{2 E_{v}}$ and $v=\frac{E_{v}+E_{\mu}-Q^{\prime}}{2 E_{v}}$. Using Eq. 1.20 and Eq. 1.18, the invariant amplitude $\mathscr{M}$ can be written as

$$
\mathscr{M}=-4 G_{F} M_{R} E_{v}\left\{\sqrt{\frac{Q^{2}}{Q^{\prime 2}}}<N^{*}\left|u F_{-}-v F_{+}\right| N>+\frac{M}{M_{R}} \sqrt{2 u v}<N^{*}\left|F_{0}\right| N>\right\},
$$

where, $F_{+}=e_{R}^{\mu} F_{\mu}, F_{-}=e_{L}^{\mu} F_{\mu}$ and $F_{0}=e_{s}^{\mu} F_{\mu}$.

The $<N^{*}\left|F_{ \pm, 0}\right| N>$ term is evaluated from the baryon harmonic oscillator model(see section 5.2). The differential cross section of a single resonance with mass $M_{R}$ and negligible width can be written as

$$
\frac{d \sigma}{d q^{2} d v}=\frac{1}{32 \pi M^{2} E_{v}^{2}} \times \frac{1}{2} \times \sum\left|\mathscr{M}\left(v N \rightarrow v N^{*}\right)\right|^{2} \delta\left(W^{2}-M_{R}^{2}\right) .
$$

Adding the three $\mathscr{M}$ terms(for the three polarizations) incoherently gives

$$
\frac{d \sigma}{d q^{2} d v}=\frac{G_{F}^{2}}{4 \pi^{2}}\left(\frac{Q^{2}}{Q^{\prime 2}}\right) \kappa\left\{u^{2} \sigma_{L}+v^{2} \sigma_{R}+2 u v \sigma_{s}\right\}
$$

where, $\kappa=\frac{M_{R}^{2}-M^{2}}{2 M}$ and $\sigma_{L, R, S}$ are the cross section for absorption of the intermediate vector boson with positive, negative or zero helicity and are proportional to $<N^{*}\left|F_{ \pm, 0}\right| N>$ and $\delta\left(W-M_{R}\right)$. 
For treating resonances with finite widths, the $\delta$ function is replaced with a Breit-Wigner factor

$$
\delta\left(W-M_{R}\right) \rightarrow \frac{1}{2 \pi} \times \frac{\Gamma}{\left(W-M_{R}\right)^{2}+\Gamma^{2} / 4},
$$

where, $\Gamma$ is the resonance width. To determine the final amplitude all of the neighboring excited resonances should be taken into account.

1.2.2.3 Deep Inelastic Scattering The DIS interactions produce a muon and a hadronic shower $(X)$

$$
v_{\mu}\left(\bar{v}_{\mu}\right) N \rightarrow \mu^{-}\left(\mu^{+}\right) X
$$

If we square the invariant amplitude of Eq. 1.13, we obtain

$$
|\mathscr{M}|^{2}=\frac{2 G_{F}^{2}}{\left(1+Q^{2} / M_{W}^{2}\right)^{2}} L_{\alpha \beta} W^{\alpha \beta},
$$

where, $M_{W}$ is the $W^{ \pm}$Boson mass, $L_{\alpha \beta}$ is the leptonic tensor and $W_{\alpha \beta}$ is the hadronic tensor. The general form of the hadronic tensor $W^{\alpha \beta}$ is expressed in terms of the four momentum vectors $q$ and $p$

$W^{\alpha \beta}=-g^{\alpha \beta} W_{1}+\frac{p^{\alpha} p^{\beta}}{M^{2}} W_{2}-i \frac{\varepsilon^{\alpha \beta \gamma \delta} p_{\gamma} p_{\delta}}{2 M^{2}} W_{3}+\frac{q^{\alpha} q^{\beta}}{M^{2}} W_{4}+\frac{p^{\alpha} q^{\beta}+p^{\beta} q^{\alpha}}{M^{2}} W_{5}+i \frac{p^{\alpha} q^{\beta}-p^{\beta} q^{\alpha}}{2 M^{2}} W_{6}$,

where, the $W$ 's are the form factors and $g^{\alpha \beta}$ is the metric tensor of Minkowski space. $\varepsilon^{\alpha \beta \gamma \delta}$ is the totally antisymmetric tensor: +1 for $\varepsilon^{0123}$ and all even permutations, -1 for odd permutations and 0 if two or more indices are the same.

The leptonic tensor $L_{\alpha \beta}$ is

$$
L_{\alpha \beta}=8\left[k_{2 \alpha} k_{1 \beta}+k_{2 \beta} k_{1 \alpha}-k_{1} \cdot k_{2} g_{\alpha \beta} \mp i \varepsilon_{\alpha \beta \gamma \delta} k_{1}^{\gamma} k_{2}^{\delta}\right]
$$

where, the minus(plus) sign is for neutrino(antineutrino) interactions. In $L_{\alpha \beta} W^{\alpha \beta}$, the $W_{6}$ term is eliminated(because there are two $W_{6}$ dependent terms that are equal and have opposite sign). The $W_{4}$ and $W_{5}$ terms are neglected because they are proportional to the lepton mass.

The differential cross section for inelastic interactions can be written as 


$$
\frac{d^{2} \sigma_{C C}^{v(\bar{v})}}{d x d y}=\frac{G_{F}^{2} M E}{\pi\left(1+\frac{Q^{2}}{M_{W}^{2}}\right)^{2}}\left[\frac{M y^{2}}{2} 2 x W_{1}\left(v, Q^{2}\right)+\left(1-y-\frac{M x y}{2 E}\right) v W_{2}\left(v, Q^{2}\right) \pm y\left(1-\frac{y}{2}\right) v x W_{3}\left(v, Q^{2}\right)\right] .
$$

The $W$ form factors can be transformed into dimensionless quantities

$$
\begin{aligned}
& F_{1}\left(x, Q^{2}\right)=M W_{1}\left(v, Q^{2}\right), \\
& F_{2}\left(x, Q^{2}\right)=v W_{2}\left(v, Q^{2}\right), \\
& F_{3}\left(x, Q^{2}\right)=v W_{3}\left(v, Q^{2}\right),
\end{aligned}
$$

where, $F_{1}, F_{2}$ and $F_{3}$ are the structure functions that contain all the information about the substructure of nucleon. The differential equation 1.29 can be re-written as

$$
\frac{d^{2} \sigma_{C C}^{v(\bar{v})}}{d x d y}=\frac{G_{F}^{2} M E}{\pi\left(1+\frac{Q^{2}}{M_{W}^{2}}\right)^{2}}\left[\frac{y^{2}}{2} 2 x F_{1}^{v(\bar{v})}\left(x, Q^{2}\right)+\left(1-y-\frac{M x y}{2 E}\right) F_{2}^{v(\bar{v})}\left(x, Q^{2}\right) \pm y\left(1-\frac{y}{2}\right) x F_{3}^{v(\bar{v})}\left(x, Q^{2}\right)\right]
$$

Often, the structure function $F_{1}\left(x, Q^{2}\right)$ is expressed in terms of $R_{L}\left(x, Q^{2}\right)$, which is the ratio of the longitudinal to transverse virtual boson absorption cross section

$$
\frac{F_{2}\left(x, Q^{2}\right)}{2 x F_{1}\left(x, Q^{2}\right)}=\frac{1+R_{L}\left(x, Q^{2}\right)}{\left(1-\frac{Q^{2}}{4 M^{2} x^{2}}\right)} .
$$

Eq. 1.31 can be re-written in terms of $F_{2}, R$ and $x F_{3}$

$$
\frac{d^{2} \sigma_{C C}^{v(\bar{v})}}{d x d y}=\frac{G_{F}^{2} M E}{\pi\left(1+\frac{Q^{2}}{M_{W}^{2}}\right)^{2}}\left[\left(\frac{y^{2}+4 M^{2} x^{2} y^{2} / Q^{2}}{2+2 R_{L}^{v(\bar{v})}\left(x, Q^{2}\right)}+1-y-\frac{M x y}{2 E}\right) F_{2}\left(x, Q^{2}\right) \pm y\left(1-\frac{y}{2}\right) x F_{3}\left(x, Q^{2}\right)\right] .
$$

Quark Parton Model (QPM) - Eq. 1.33 is a general form of the cross section for inelastic interactions. The naive QPM[35] assumes that the nucleon is made up of non-interacting pointlike constituents called partons (quarks). The model is valid in the "infinite momentum" frame, in which the time scale of interactions(strong interaction) between partons is much larger than the 
collision time. In the limit $Q^{2} \rightarrow \infty$, the structure functions depend only on the Bjorken scaling variable $x$

$$
\lim _{Q^{2} \rightarrow \infty} F_{i}\left(x, Q^{2}\right) \rightarrow F_{i}(x)
$$

This property is known as the Bjorken scaling. The scaling variable $x$ can be thought of as the fraction of the initial nucleon momentum being carried by the struck quark. In the QPM model, the structure functions can be written in terms of the Parton Distribution Functions(PDFs). For neutrino proton interactions we have

$$
\begin{aligned}
& F_{2}^{v p}(x)=2 x[d(x)+\bar{u}(x)+s(x)+\bar{c}(x)], \\
& x F_{3}^{v p}(x)=2 x[d(x)-\bar{u}(x)+s(x)-\bar{c}(x)],
\end{aligned}
$$

where, the PDFs for up $(u(x))$, down $(d(x))$, strange $(s(x))$ and charm $(c(x))$ can be interpreted as the probability that a given parton will carry a fraction $x$ of the nucleon's four momentum. The neutron and proton quark distributions can be related using isospin invariance

$$
\begin{aligned}
& d(x) \equiv d^{p}(x)=u^{n}(x), \quad \bar{d}(x) \equiv \bar{d}^{p}(x)=\bar{u}^{n}(x), \\
& u(x) \equiv u^{p}(x)=d^{n}(x), \quad \bar{u}(x) \equiv \bar{u}^{p}(x)=\bar{d}^{n}(x) .
\end{aligned}
$$

The structure functions for neutrino neutron interactions are

$$
\begin{aligned}
& F_{2}^{v n}(x)=2 x[u(x)+\bar{d}(x)+s(x)+\bar{c}(x)], \\
& x F_{3}^{v n}(x)=2 x[u(x)-\bar{d}(x)+s(x)-\bar{c}(x)] .
\end{aligned}
$$

The structure function for an isoscalar nucleon is calculated by taking the average of Eq. 1.35 and Eq. 1.36

$$
\begin{aligned}
& F_{2}^{v N}(x)=x[u(x)+d(x)+2 s(x)+\bar{u}(x)+\bar{d}(x)+2 \bar{c}(x)], \\
& x F_{3}^{v N}(x)=x[u(x)+d(x)+2 s(x)-\bar{u}(x)-\bar{d}(x)-2 \bar{c}(x)] .
\end{aligned}
$$


The structure function for antineutrinos can be written by using the fact that they interact with positively charged quarks only(using the assumption $s(x)=\bar{s}(x), c(x)=\bar{c}(x)$ )

$$
\begin{aligned}
& F_{2}^{\bar{v} N}(x)=F_{2}^{v N}(x), \\
& x F_{3}^{\bar{v} N}(x)=x F_{3}^{v N}(x)-4 x[s(x)-c(x)] .
\end{aligned}
$$

In this simplified Leading $\operatorname{Order}(\mathrm{LO})$ model, the structure functions(here $\left.x F_{3}=\frac{1}{2}\left(x F_{3}^{v}+x F_{3}^{\bar{v}}\right)\right)$ can be expressed in terms of the PDFs $q_{i}\left(x, Q^{2}\right)$

$$
\begin{aligned}
& 2 x F_{1}\left(x, Q^{2}\right)=F_{2}\left(x, Q^{2}\right), \\
& \left.F_{2}=\sum_{i=u, d . .} x q_{i}\left(x, Q^{2}\right)+x \bar{q}_{i}\left(x, Q^{2}\right)\right], \\
& \left.x F_{3}=\sum_{i=u, d . .} x q_{i}\left(x, Q^{2}\right)-x \bar{q}_{i}\left(x, Q^{2}\right)\right],
\end{aligned}
$$

where the summation is over the interacting quarks, up, down, sea and charm.

Quantum Chromo Dynamics(QCD) - The QPM explains the scaling behavior observed in DIS experiments at high $Q^{2}$. At low $Q^{2}$, violations of Bjorken scaling have been observed that lead to a $Q^{2}$ dependence of the structure functions. The QPM assumes that the partons are noninteracting and quasi-free in a nucleon. The theory of QCD [59] takes into account interactions between partons via the exchange of gluons.

In QCD, quarks and gluons carry color charge which means they undergo strong interactions. The self interaction of gluons anti-screens the color charge which leads to the confinement of quarks within a hadron. The strong force between quarks increases with distance and at short distances (high $Q^{2}$ ) they behave like free particles and approach "asymptotic freedom". Asymptotic freedom allows the quark-lepton cross section to be calculated as a perturbative series in terms of the strong coupling constant $\alpha_{s}$. Fig. 1.2 shows the leading order(LO) diagrams that are proportional to $\alpha_{s}^{1}$. Terms proportional to $\alpha_{s}^{2}$ are referred to as next to leading order(NLO).

Non-perturbative QCD effects - At lower $Q^{2}\left(<1 \mathrm{GeV}^{2}\right)$, the perturbative approximation breaks down and the resolving power of the virtual boson is not enough to probe individual partons. The non-perturbative QCD effects are collectively termed as higher-twist effects and examples include, multiparton scattering and target mass effects. Lepton nucleon scattering at low $Q^{2}$ can 

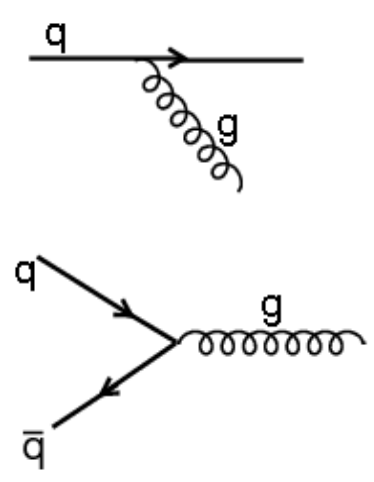
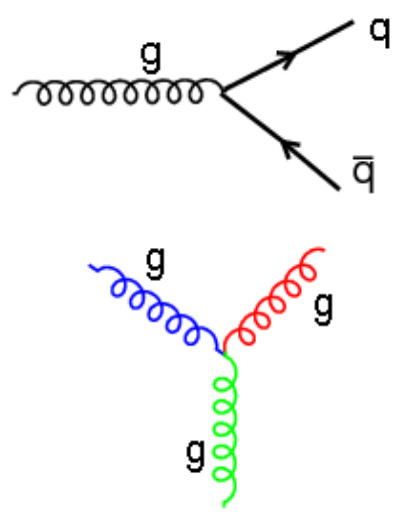

Figure 1.2: LO diagrams that are included in QCD calculations.

involve multiple partons (Fig. 1.3). The contribution from this effect is suppressed by a factor of $1 / Q^{2}$ compared to those from perturbative QCD which makes them important at low $Q^{2}$. In our derivation of the DIS cross section, parton mass was neglected but it becomes important at low $Q^{2}$ and high $x$. To account for target mass, the scaling variable $x$ is replaced by an effective scaling variable $\xi[38]$

$$
\xi=\frac{2 x}{1+k}
$$

where, $k=\left(1+4 M^{2} x^{2} / Q^{2}\right)$. 


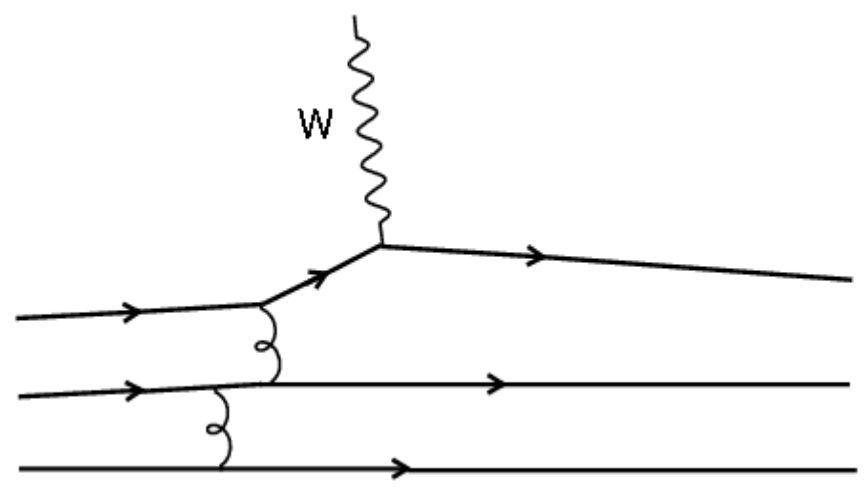

Figure 1.3: Feynman diagram for multiparton scattering. 


\subsection{THE NUMI BEAM AND MINOS DETECTORS}

The main physics goal of the MINOS(Main Injector Neutrino Oscillation Search) experiment is to measure the neutrino oscillation parameters $\Delta m_{32}^{2}$ and $\sin ^{2} 2 \theta_{23}$ by studying the disappearance of muon neutrinos in the NuMI(Neutrinos at Main Injector) beam. NuMI is an intense neutrino beam produced from protons extracted from the Main Injector accelerator at Fermilab, Illinois. To measure the oscillation parameters, there are two identical detectors, the near detector located at Fermilab, Illinois which serves as a "reference" detector and the far detector located about $735 \mathrm{~km}$ downstream in a mine at Soudan, Minnesota. Both the detectors are tracking calorimeters made of iron planes alternating with scintillator planes. The first section describes the NuMI beam and the next two sections give a description of the MINOS near detector and the far detector.

\subsection{NUMI BEAM}

The NuMI beamline shown in Fig. 2.1 at Fermilab produces a powerful muon neutrino beam from interactions of a $120 \mathrm{GeV}$ proton beam (extracted from the Main Injector accelerator) on a graphite target. The proton beam is aimed downward by an angle of $58 \mathrm{mrad}$ to point at the far detector that is located in an underground mine. Protons are extracted in a $10 \mu s$ spill with a typical intensity of $2.1 \times 10^{13}$ Protons on Target(PoT)/spill from the Main Injector(MI). The MI accelerates protons in seven batches, out of which five are directed towards the NuMI beamline. Fig. 2.2 shows the five batches in a typical spill.

Charged mesons(mostly pions and some kaons) that are produced when the proton beam impinges on a graphite target pass through a pair of magnetic horns that focus them in the direction of the proton beam. The horns are made of co-axial conductors that produce a toroidal magnetic field. 


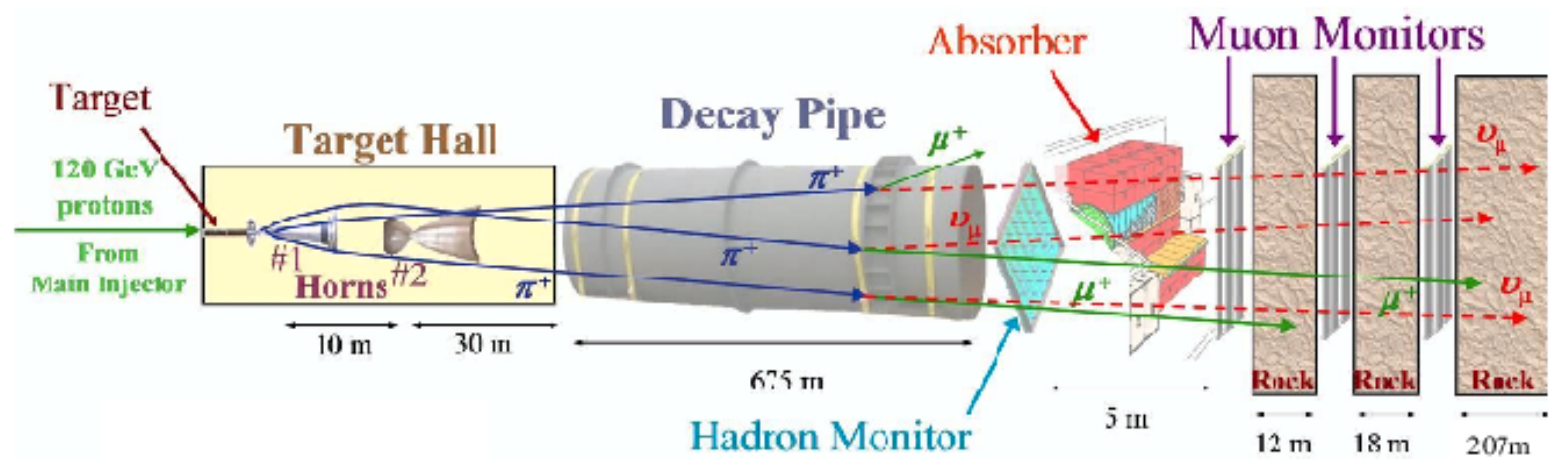

Figure 2.1: Layout of the Neutrino beamline. Protons from the Main Injector hit a graphite target producing charged mesons. The charged mesons are focused by a pair of magnetic horns and subsequently decay to produce muons and muon neutrinos. The muons and uninteracted hadrons are absorbed and the neutrinos continue to the MINOS Near Detector [61].

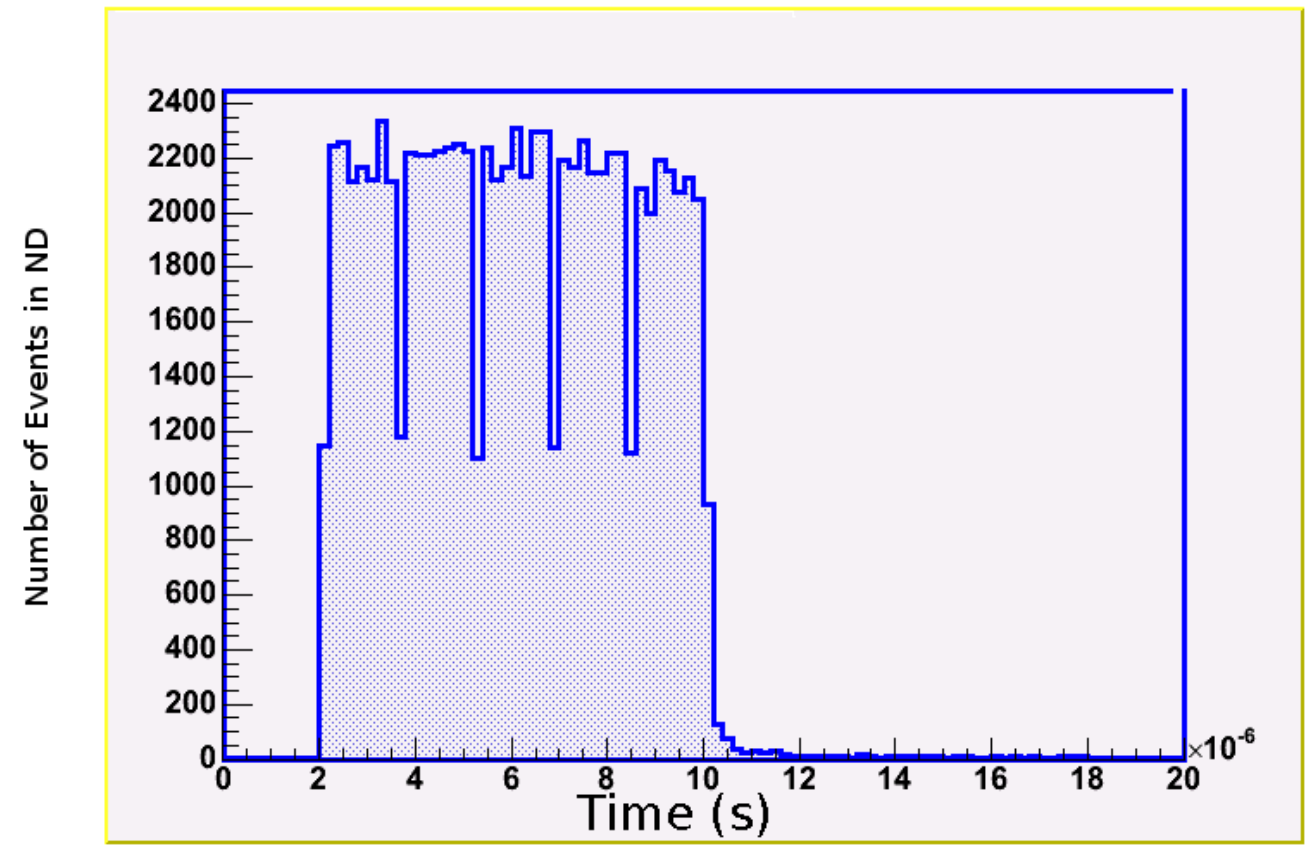

Figure 2.2: Number of events in the near detector as a function of time. The five batch structure in a $10 \mu s$ spill can be clearly seen. 
The parabolic shape of the inner conductor(as shown in Fig. 2.1) causes the field to act as a lens, where the focal length is proportional to the meson momentum and the horn current. This allows selection of a certain momentum window through focusing. The mesons that are under focused or over focused are further focused by the second horn.

The mesons enter a $675 \mathrm{~m}$ long decay pipe, where about $10 \%$ of the mesons decay to muons and muon neutrinos

$$
\begin{aligned}
& \pi^{-(+)} \rightarrow v_{\mu}\left(\bar{v}_{\mu}\right)+\mu^{-(+)} \\
& K^{-(+)} \rightarrow v_{\mu}\left(\bar{v}_{\mu}\right)+\mu^{-(+)} .
\end{aligned}
$$

Some of the kaons and muons also produce electron neutrinos. The predicted composition of the neutrino beam after interaction with the detector is $92.9 \% v_{\mu}, 5.8 \% \bar{v}_{\mu}$ and $1.3 \% v_{e}+\bar{v}_{e}$. In the standard data collecting mode, the parabolic horns focus the positively charged mesons which leads to a much higher contribution of the $v_{\mu} \mathrm{s}\left(\right.$ compared to $\left.\bar{v}_{\mu} \mathrm{s}\right)$ in the neutrino beam.

The decay pipe is followed by a hadron absorber which absorbs undecayed mesons, muons and uninteracted protons. The remaining muons range out in $240 \mathrm{~m}$ of rock after the absorber and the neutrino beam reaches the ND located $1.04 \mathrm{~km}$ downstream of the target. The beam monitoring system, which consists of hadron and muon monitors is used to measure the spatial distribution of muons and hadrons. The monitors are ionization chambers; a hadron monitor is located upstream of the absorber, a muon monitor is located immediately after the absorber and at two more stations in the rock that follows the absorber.

The neutrino energy spectrum is determined by the relative position of the target and horns and the value of the horn current. Most of the data were collected in the "low energy" LE10/185 kA mode in which the target is placed $10 \mathrm{~cm}$ upstream from the first focusing horn and the horn current is $185 \mathrm{kA}$. Fig. 2.3 shows the simulated flux of the $v_{\mu}$ and $\bar{v}_{\mu}$ beam. The $v_{\mu}$ beam peaks between 3-4 GeV with a long tail. Since the negatively charged mesons(which produce the $\bar{v}_{\mu} \mathrm{s}$ ) are defocused by the horns, only those negative mesons that are relatively high energy are able to pass through the horns, this makes the $\bar{v}_{\mu}$ beam higher in energy and less sharply focused. 


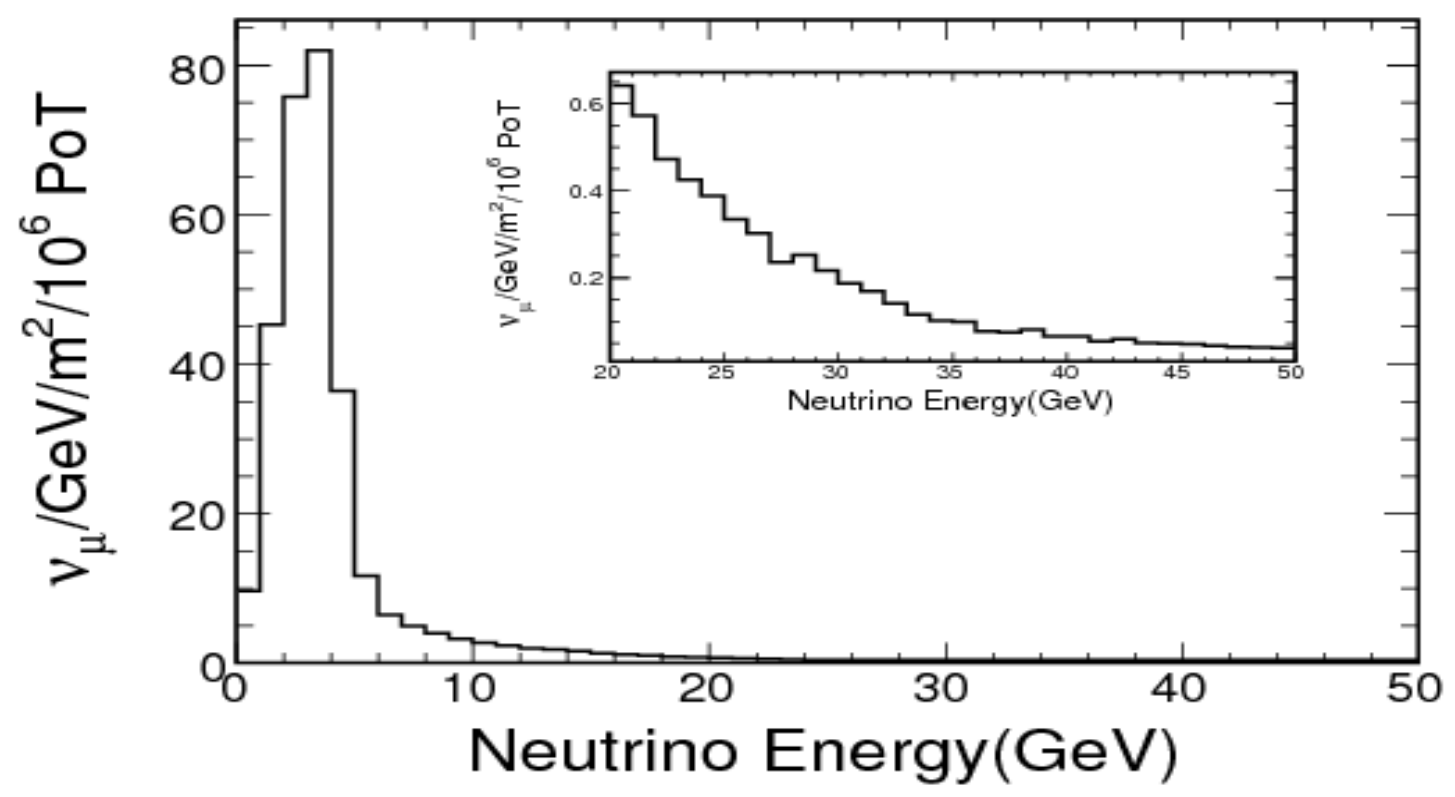

(a)

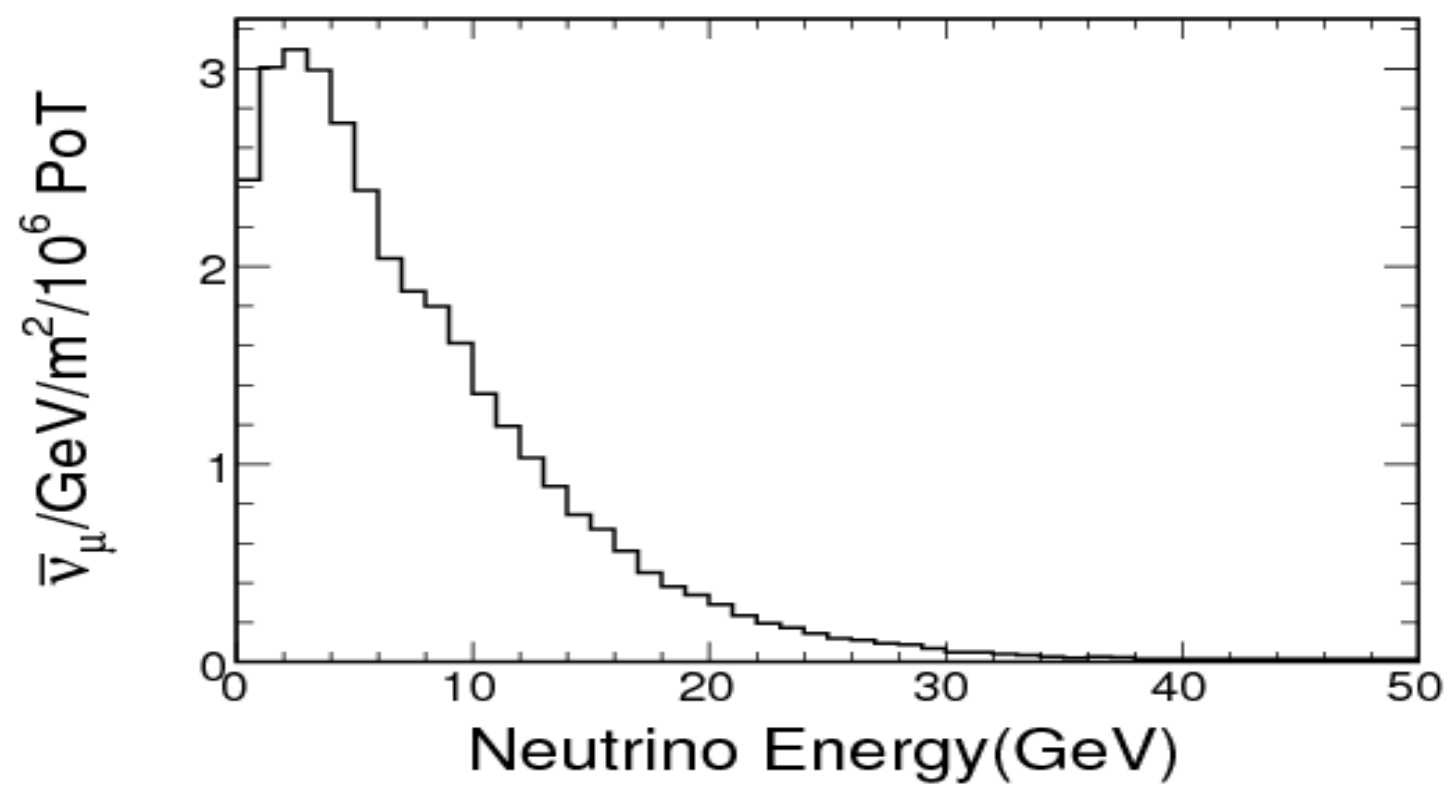

(b)

Figure 2.3: Simulated flux spectrum of the low energy $v_{\mu}$ (a) and $\bar{v}_{\mu}$ beam(b) (which is $5.8 \%$ of the neutrino beam) in units of number of events $/ \mathrm{GeV} / \mathrm{m}^{2} / 10^{6}$ PoT. The inset figure shows the $v_{\mu}$ spectrum above $20 \mathrm{GeV}$. The PoT in the label stands for protons on target. 


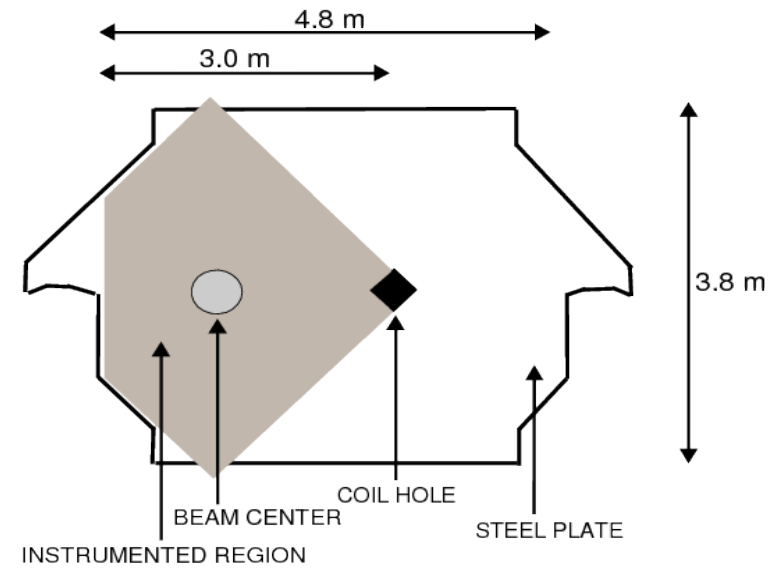

Figure 2.4: Transverse view of a Near Detector plane where the shaded region shows an instrumented active scintillator plane.

\subsection{NEAR DETECTOR}

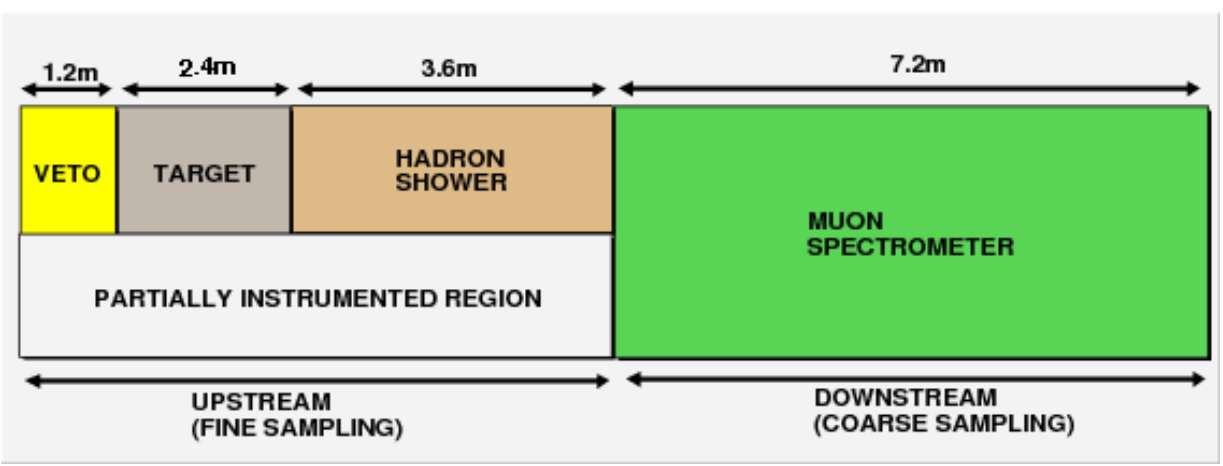

Figure 2.5: Longitudinal view of the near detector showing the different segmentations.

The analysis presented in this thesis has been performed on data obtained from the Near Detector(ND). The ND shown in Fig. 2.4 is located $1 \mathrm{~km}$ from the NuMI target and at a depth of $90 \mathrm{~m}$. It has a "squashed" octagonal structure which is $4.8 \mathrm{~m}$ wide, $3.8 \mathrm{~m}$ high, $16.6 \mathrm{~m}$ long and has a mass of 0.98 kton. The detector has a toroidal magnetic field of average strength $1.3 \mathrm{~T}$. The ND consists of 282 steel planes, $2.54 \mathrm{~cm}$ thick, of which 152 are instrumented with $1 \mathrm{~cm}$ thick scintillator planes. 


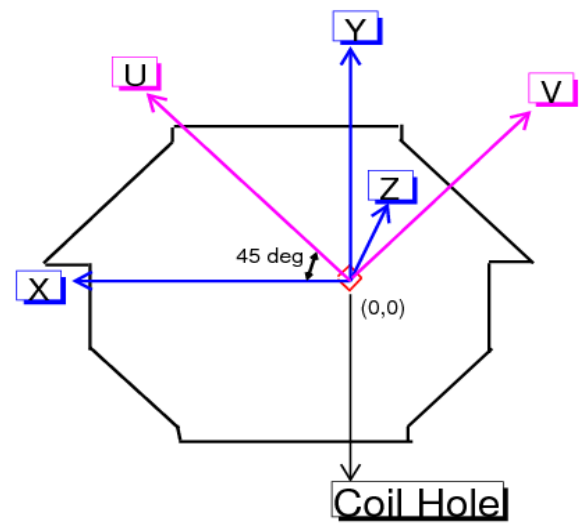

Figure 2.6: The U-V co-ordinate system used at the near detector. The coil hole is at the center of the co-ordinate system.

It is divided into four longitudinal sections as shown in Fig. 2.5. These are listed below, starting from the upstream end of the detector

1. Veto - The first $1.2 \mathrm{~m}$ of the detector is called the "veto" region. This region is used to identify incoming charged particles which otherwise might be mis-identified as neutrino interactions.

2. Target - The next region starting at $1.2 \mathrm{~m}$ and ending at $3.6 \mathrm{~m}$ is called the "target" region. Neutrino interactions beginning in this part are included in the fiducial volume.

3. Hadron Shower - The target region is followed by the "hadron shower" region staring at 3.6 $\mathrm{m}$ and ending at $7.2 \mathrm{~m}$. This region is long enough to contain hadron showers from neutrino interactions occurring in the target region. The first three regions, veto, target and hadron shower are collectively called the "calorimeter" which includes the first 120 planes of the detector.

4. Muon Spectrometer - The remaining $7.2 \mathrm{~m}$ is the muon spectrometer. This is needed to measure the muon charge and momentum from curvature in the magnetic field. 


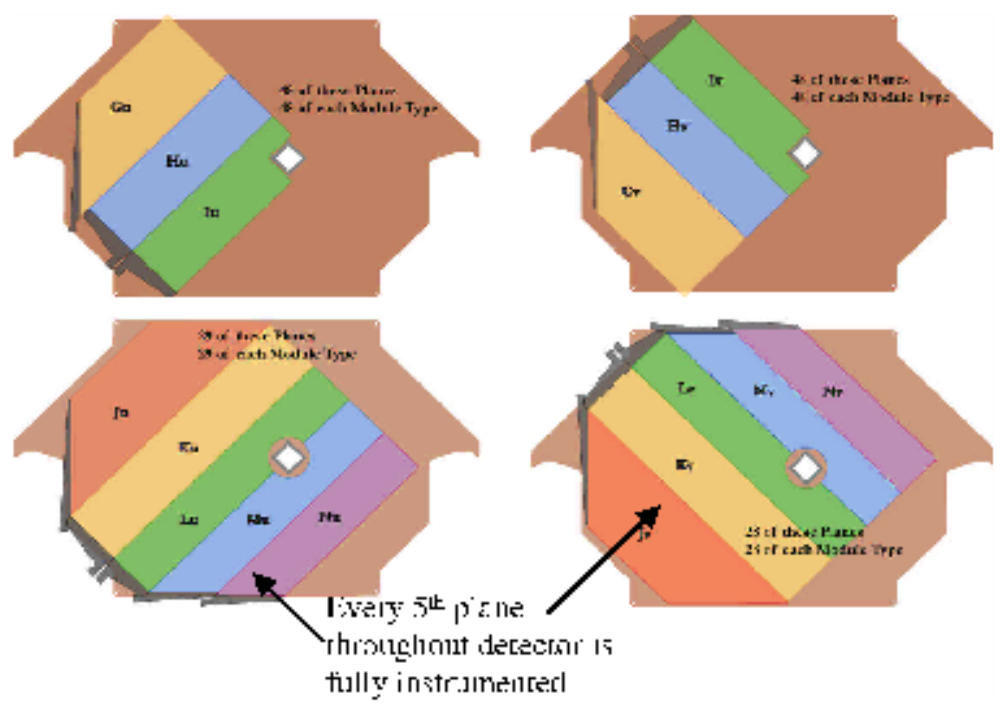

Figure 2.7: A partially instrumented plane is shown on the top and a fully instrumented plane on the bottom. The u-view is shown on the left and the v-view is shown on the right. Each partially(fully) instrumented plane is divided into three(five) scintillator modules, namely G,H,I (J,K,L,M,N).

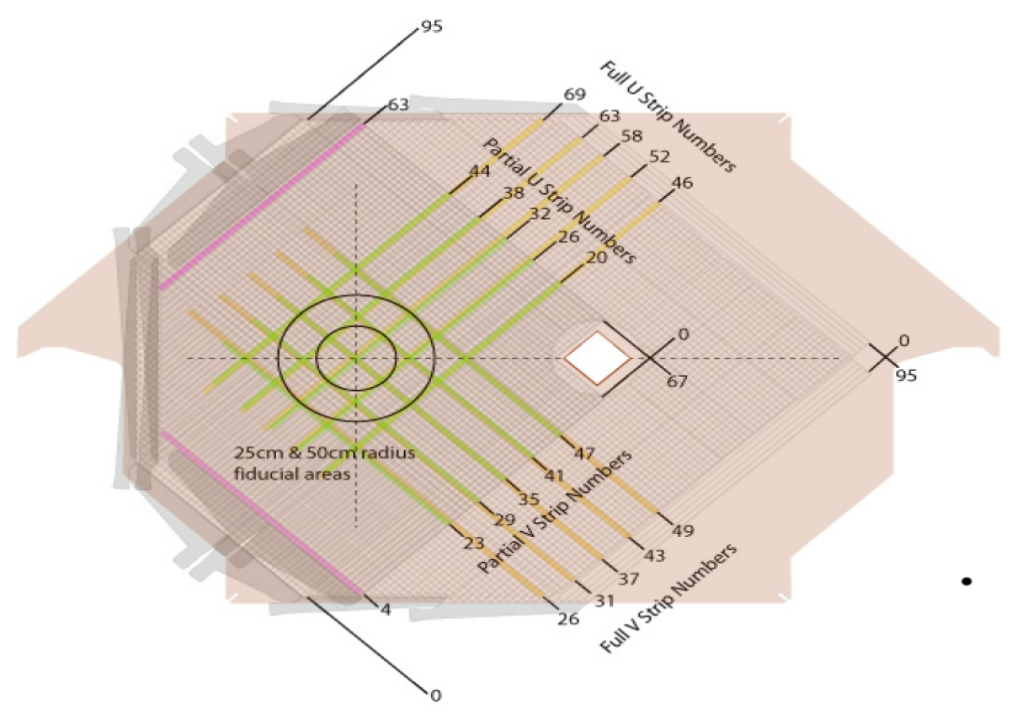

Figure 2.8: Detailed arrangement of the strips in a typical near detector $\mathrm{U}$ and $\mathrm{V}$ plane for a partially and a fully instrumented plane. 
Each scintillator strip is $4.1 \mathrm{~cm}$ wide and $1 \mathrm{~cm}$ thick. The scintillator strips in the instrumented planes are oriented along the $\mathrm{U}$ and $\mathrm{V}$ axis alternatively which are rotated by $\pm 45^{0}$ with respect to the vertical direction. Fig. 2.6 shows the U-V co-ordinates with respect to the $\mathrm{X}-\mathrm{Y}$ co-ordinates. The coil hole is at the origin of the co-ordinate system. The strips are placed along $\mathrm{U}$ and $\mathrm{V}$ directions to allow measurement of atmospheric neutrino interactions (which come at steeper angles to the horizontal) and also because it leaves room below to install the readout system.

Every 5th plane throughout the detector is fully instrumented with 96 adjacent scintillator strips. A group of adjacent strips are enclosed in aluminum covers and referred to as modules. The fully instrumented planes have 5 scintillator modules(J,K,L,M,N) each as shown in Fig. 2.7(bottom row). The calorimeter region has partially instrumented planes(64 scintillator strips) in between the fully instrumented planes that have three modules each (G,H,I). A detailed diagram of the strip arrangement in both the $\mathrm{U}$ and $\mathrm{V}$ view in a given plane is shown in Fig. 2.8. In the spectrometer there is no readout of scintillators between the fully instrumented planes. Light collected from the scintillators are carried to Photo Multiplier Tubes(PMT) via optical fibers. The PMT signals are readout by an electronic readout and the data is subsequently stored by the Data Acquisition system.

\subsection{SCINTILLATOR}

Each scintillator strip is made of plastic polystyrene doped with PPO and POPOP organic fluor and is jacketed in a reflective layer made of $85 \%$ polystyrene and $15 \% \mathrm{TiO}_{2}$ by weight. As a charged particle travels through the scintillator, it excites the fluor which emits the absorbed energy in the

form of ultra violet photons. These photons are collected by $1.2 \mathrm{~mm}$ diameter wavelength shifting (WLS) fibers that are embedded in a $2 \mathrm{~mm}$ deep groove that runs along the length of each strip as shown in Fig. 2.9(a). The light yield of a strip can be parametrized as

$$
I=I_{0} e^{-x / \lambda_{\text {atten }}}
$$

where, $I_{0}$ is the initial intensity, $\mathrm{x}$ is the distance along the fiber and $\lambda_{\text {atten }}$ is the attenuation length of the WLS fiber. The typical attenuation length is about $6.5 \mathrm{~m}$ which is acceptable because the 
average distance traveled by photons is about $4 \mathrm{~m}$.

The WLS fibers absorb and re-emit the ultra violet light from scintillation as green light so that it can be detected by the Photo Multiplier Tubes(PMT). The WLS fibers, which are read out from one end only, lead to optical connectors. From the optical connectors the light is carried by clear optical fibers which have a longer attenuation length of $11 \mathrm{~m}$. The optical fibers lead to dark boxes that house the PMTs. The dark boxes enclose the connection between the clear optical fibers, PMTs and the front-end electronics. Fig. 2.9(b) shows a picture of the optical readout at the ND.

\subsection{PHOTO MULTIPLIER TUBES}

The ND uses 210 Hamamatsu R-5900-00 M64 phototubes shown in Fig.2.10, which are multianode PMTs that have 64 pixels each of size $2 \mathrm{~mm} \times 2 \mathrm{~mm}$. Each pixel acts as a single anode phototube. The incident light strikes the photo cathode of the PMT and releases photo electrons that are attracted to a series of dynodes which are at a positive voltage relative to the cathode. The number of electrons get multiplied at each dynode through secondary emission. At the end, there is an anode which records a sharp current pulse caused by the accumulation of charge.

The quantum efficiency(Q.E.), defined as the probability that one photon releases one photo electron, is a function of wavelength and is $20 \%$ for the MINOS ND case. The electrons are accelerated through a 12 stage dynode inside the PMT and the average voltage between the photocathode and the anode is about $800 \mathrm{~V}$. The typical gain(number of electrons at the anode/initial

photo electron at the cathode) of a M-64 PMT is $1 \times 10^{6}$ and it has a linear response for input pulses less than 100 photoelectrons(p.e.).

The muon spectrometer has 4:1 electronic multiplexing in which four strips(which are connected to 4 separate PMT channels) are read out by one electronic channel. This information has to be "demultiplexed" in order to reconstruct the different particle tracks inside the detector (see section 4.0.4). 


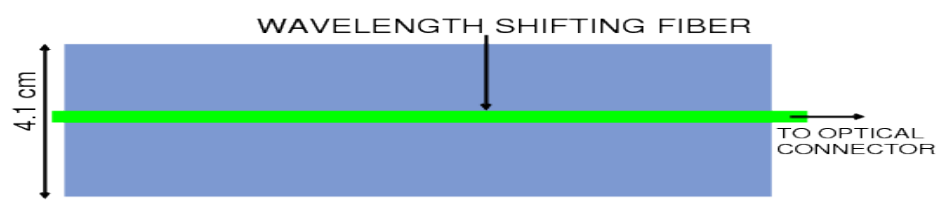

(a)

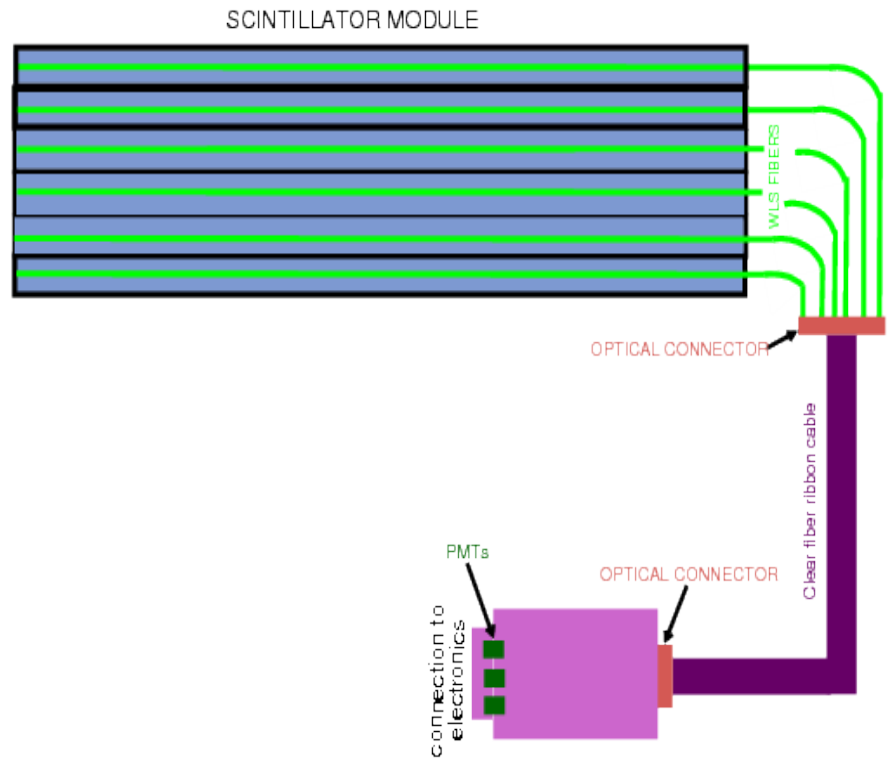

(b)

Figure 2.9: A scintillator strip used in the near detector(a) and schematic of the optical connections in a scintillator module(b). 

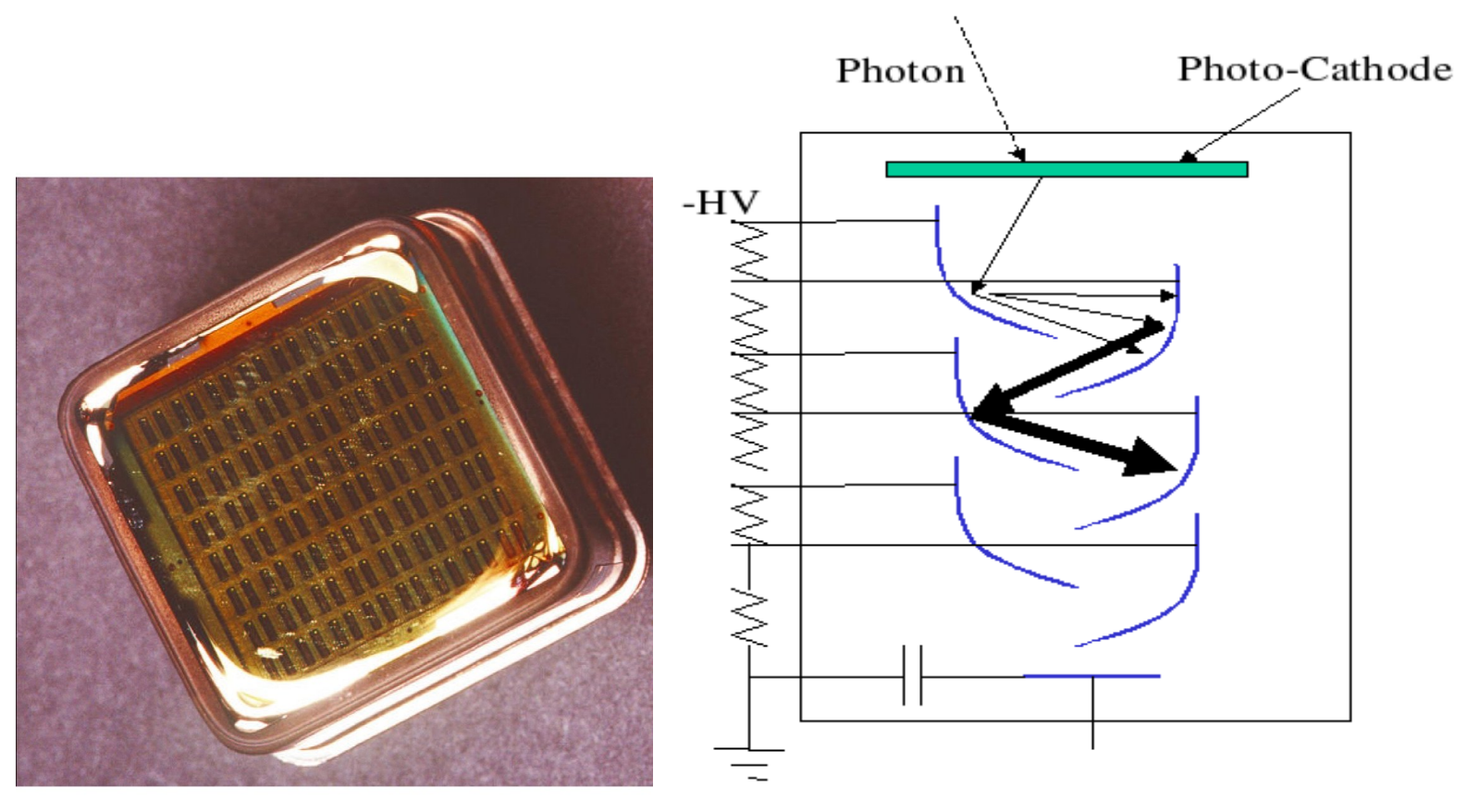

Figure 2.10: Face of M-64 phototube. Each of the 64 pixels ( $2 \mathrm{~mm}$ x $2 \mathrm{~mm}$ ) receive the output of a single scintillator strip. (a) Schematic of a PMT (b). 


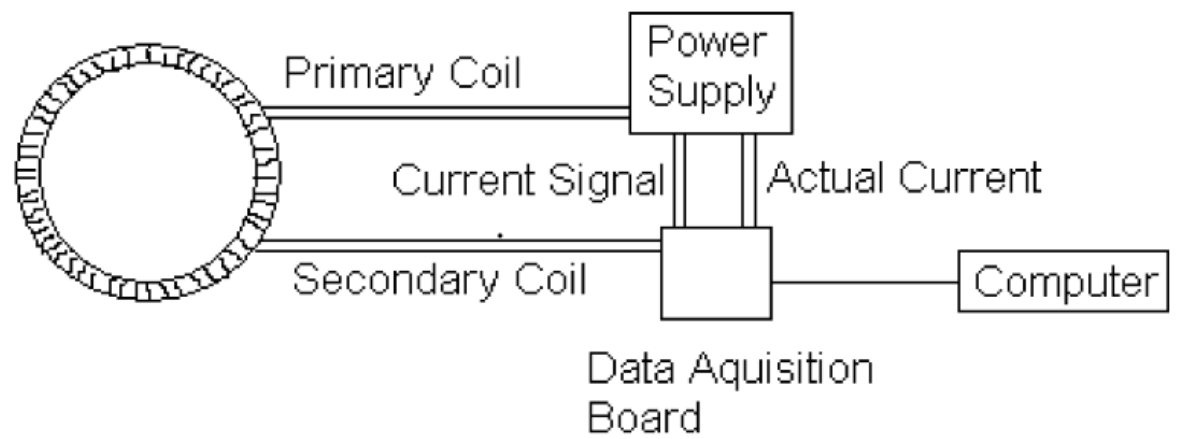

Figure 2.11: The setup used for measuring the B-H curve on a batch of steel that is used in the Near Detector.

\subsection{MAGNETIC FIELD}

The magnetic field is used to distinguish the $\mu^{-}$s from $\mu^{+}$s coming from $v_{\mu}$ and $\bar{v}_{\mu}$ charged current interactions respectively and to measure the momentum of muons from their curvature. The toroidal magnetic field in the iron detector is generated by an aluminum coil that passes through the hole at $(0,0)$ of the co-ordinate system shown in Fig. 2.6. The coil carries a current of 40,000 A-turns with each of the 48 turns carrying 833 A. The coil hole is placed off-center horizontally and the detector is placed so that the beam is incident at $1.5 \mathrm{~m}$ from the coil hole.

The magnetic field map is estimated using an ANSYS-Finite Element Analysis(FEA) program[1]. The inputs to the FEA program are the detailed geometry of a near detector plane along with the coil hole geometry and a vendor provided B-H curve. The vendor measured the B-H curve by constructing a test ring from a sample of steel used in the detector. The setup is shown in Fig 2.11. It has a computer controlled power supply to magnetize the steel through a primary coil. When the current through the primary coil is changed, the magnetic field in the toroid induced a current in the secondary that is read by the computer. Fig. 2.12 shows the measured B-H curve and Fig. 2.13(a) shows the model of the field map constructed with this B-H curve.

The field has been designed such that the average value is $1.3 \mathrm{~T}$ in the fiducial region.The asymmetric nature of the field is due the fact that the coil hole is off-center. The uninstrumented 


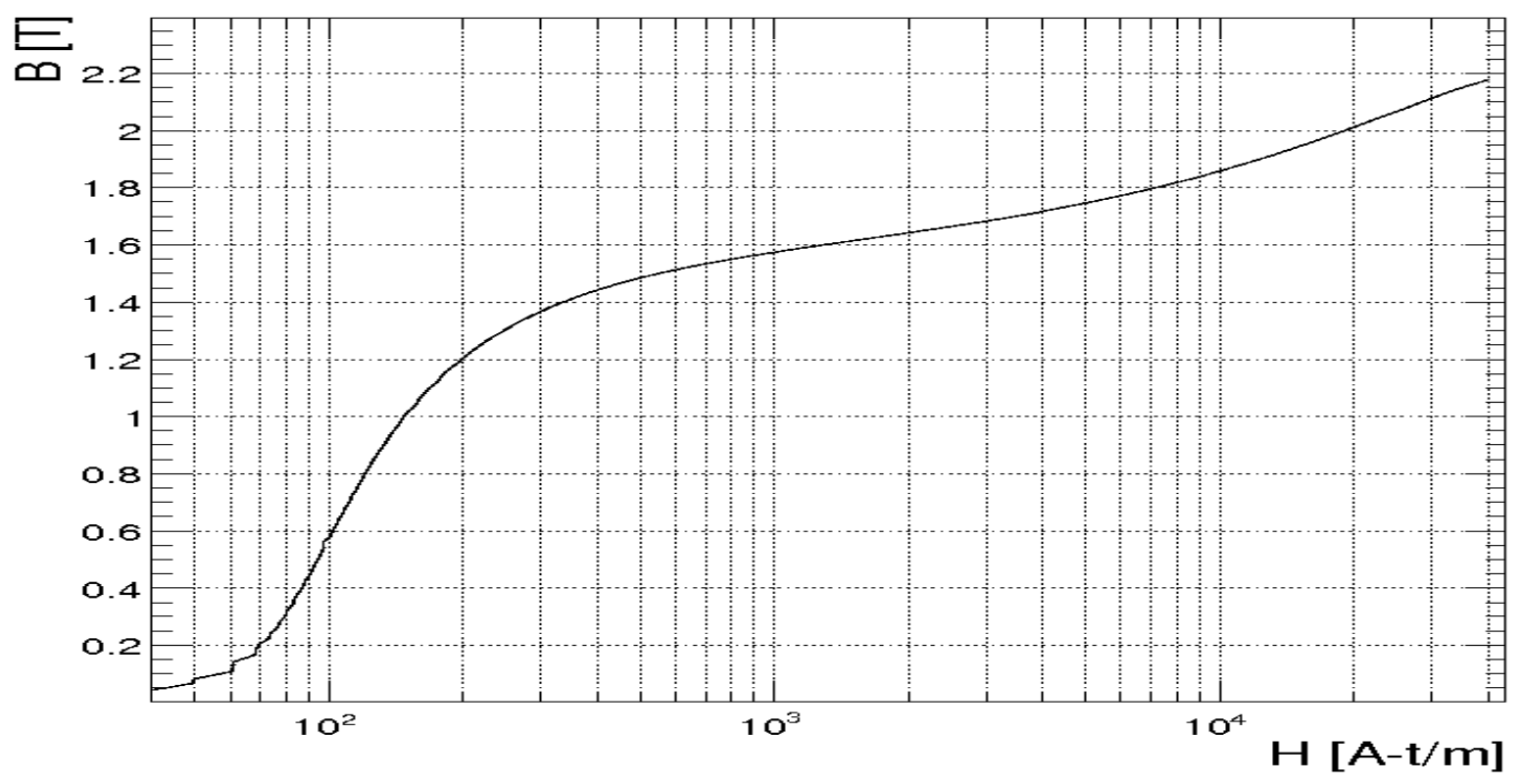

Figure 2.12: The vendor provided B-H curve for the Near Detector.

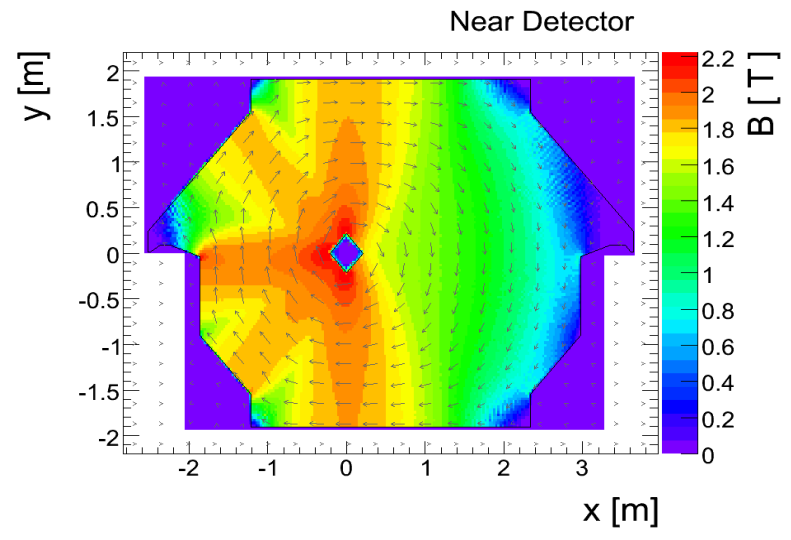

(a)

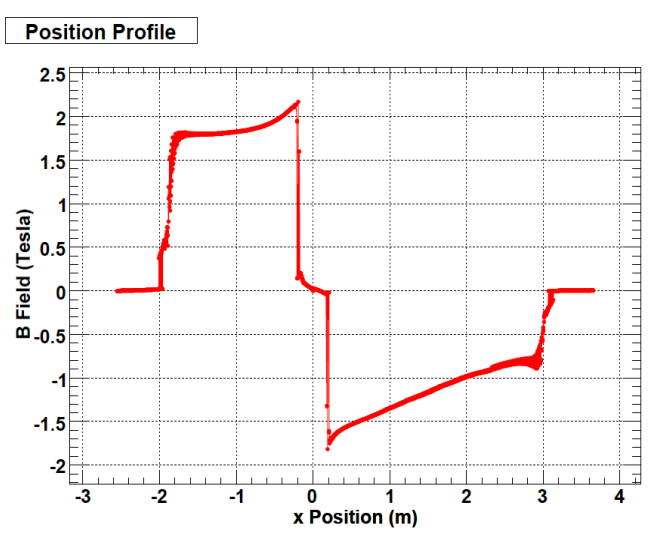

(b)

Figure 2.13: The Near Detector field map estimated from the FEA program(a). The direction of the azimuthal magnetic field is shown by arrows. Change in the magnitude of the magnetic field as a function of $\mathrm{x}(\mathrm{m})$ at a constant $\mathrm{y}$ of $-10 \mathrm{~cm}(\mathrm{~b})$. 
region(on the left of the coil hole) has a higher magnetic field compared to the instrumented region. The same amount of magnetic flux passes through both the instrumented region as well as the uninstrumented region, but the area of the former is larger than that of the latter which makes the magnetic field higher in the uninstrumented region. Fig. 2.13(b) shows the variation in the magnetic field as a function of $\mathrm{x}$ position(m) at a constant value of $\mathrm{y}(-10 \mathrm{~cm})$. In the instrumented region $(x>0 m)$ the magnetic field decreases with a constant gradient from a value of about $1.5 \mathrm{~T}$ around the coil hole $(0 \mathrm{~m})$ to $1.0 \mathrm{~T}$ around the edge of the edge of the instrumented region $(3.0 \mathrm{~m})$.

The magnetic field has been directly measured at some positions in the detector in order to compare the predicted field value to the measured value. The measurement has been performed by winding an induction coil around the planes at certain positions which are shown in Fig. 2.14. The voltage across the secondary induction coil and the current in the primary coil can be related to the average magnetic field $\mathrm{B}(\mathrm{T})$ and the magnetic induction $\mathrm{H}(\mathrm{A}-\mathrm{t} / \mathrm{m})$. This study was performed for two positions "1"(outside the fiducial volume) and "6"(inside the fiducial volume) and the result was averaged over several planes. Table 2.1 shows the result of this study from which we can conclude that the measured and predicted magnetic field agreement is better than $2 \%$.

This $2 \%$ difference between the measured and predicted value is caused by plane to plane variation of the magnetic field that is not taken into account by the FEA simulation. The steel in the detector comes from different batches or "heats" of steel that have a slightly different chemical composition and hence magnetic properties. A given plane is constructed from a single batch of steel, but the different batches are distributed throughout the detector. These batches have slightly different $\mathrm{BH}$ distributions but the FEA simulation uses the median BH curve. In addition to chemical variations, the planes can also have slightly different thicknesses. The proximity of the return legs of the magnetic coil to the end of the detectors causes an additional effect on the field of the end planes on either side of the detector.

\subsection{READOUT AND DATA ACQUISITION}

The electronic readout digitizes the charge and time of each PMT signal. The electronics have been designed to record neutrino interactions throughout a spill with no deadtime and with a timing 


\begin{tabular}{|c|c|c|c|c|}
\hline Position & \# of planes & Measured $<\mathrm{B}>(\mathrm{T})$ & Predicted $<\mathrm{B}>(\mathrm{T})$ & Ratio \\
\hline 1 & 10 & 1.82 & 1.83 & 0.997 \\
\hline 6 & 8 & 1.19 & 1.20 & 0.989 \\
\hline
\end{tabular}

Table 2.1: Comparison of measured and predicted magnetic field value. The first column shows the two positions(" 1 " and "6") and the second column shows the number of planes for which the field was measured. The third and fourth columns show the measured and predicted average magnetic field in the area over which the induction coil was wound. The fifth column shows the ratio of measured to predicted field.

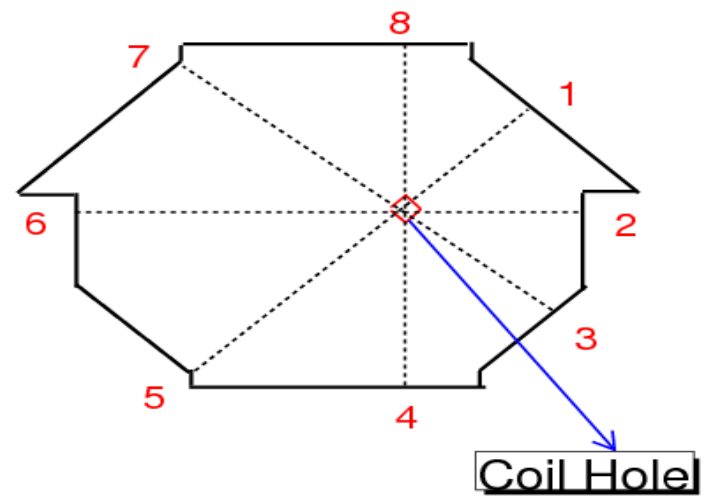

Figure 2.14: The positions at which induction coils are wound to do a direct measurement of the magnetic field at the Near Detector. 
resolution that allows separation of overlapping events. The electronic channel ID, timestamp and the ADC(Analogue-to-Digital) value of the digitizations are stored in a pair of buffers from where they are read out by the Data Acquisition System(DAQ). The DAQ and timing system synchronize with the electronics and continuously readout the front-end-electronics.

\subsubsection{Electronic Readout}

The electronic readout is based on a QIE (Charge to Current Encoder) chip that consists of a current splitter, gated integrator and range selector as shown in Fig. 2.15. The current from the PMT is split by the current splitter into eight binary weighted ranges $\mathrm{I} / 2, \mathrm{I} / 4 \ldots \mathrm{I} / 256$. Splitting the current gives the QIE chip sensitivity over a large range of incoming charge from $10 \mathrm{fC}$ to $10 \mathrm{pC}$. The current in each branch is used to charge a capacitor that serves as the integrator. After integration the voltage across the capacitors are fed to an ADC converter through a range selector. The range selector selects the voltage that lies within the input range of the ADC converter with the help of a comparator. It also outputs the selected voltage range in a three bit digital format and additional two-bits called the CAPID which identifies which of the four circuits have been used. The ADC converts the analogue voltage into a 8-bit flash ADC value. At the end of the clock cycle(19 ns), the capacitors are discharged to get ready for the next cycle. The QIE chip has been made deadtime free by using four sets of integrator/range selector which are parallel and are 1/4 cycle out of phase with one another. The output from the ADC is stored in a FIFO which is a buffer that can store digitizations from 1000 clock cycles $(19 \mu \mathrm{s})$ before it is read out.

The near detector has an event rate of about 20 events in one beam spill that leads to overlapping events. In order to effectively separate these events the readout has a timing resolution of $19 \mathrm{~ns}$ which matches the $53 \mathrm{MHz}$ RF rate of the Main Injector. A clock system synchronizes the Main Injector frequency, electronic readout and the DAQ. Every digit bears a timestamp from this clock system which resets to 0 across the detector once per second. This timestamp is combined with the time elapsed in seconds to obtain the absolute time of each digit to $19 \mathrm{~ns}$.

The smallest front-end channel unit is a MENU(MINOS Electronics for Neutrinos) board that holds a single QIE chip, ADC converter and a FIFO. Sixteen MENU boards are placed on a single MINDER (MINOS Near Detector Readout) which controls the power and clock signals to the 


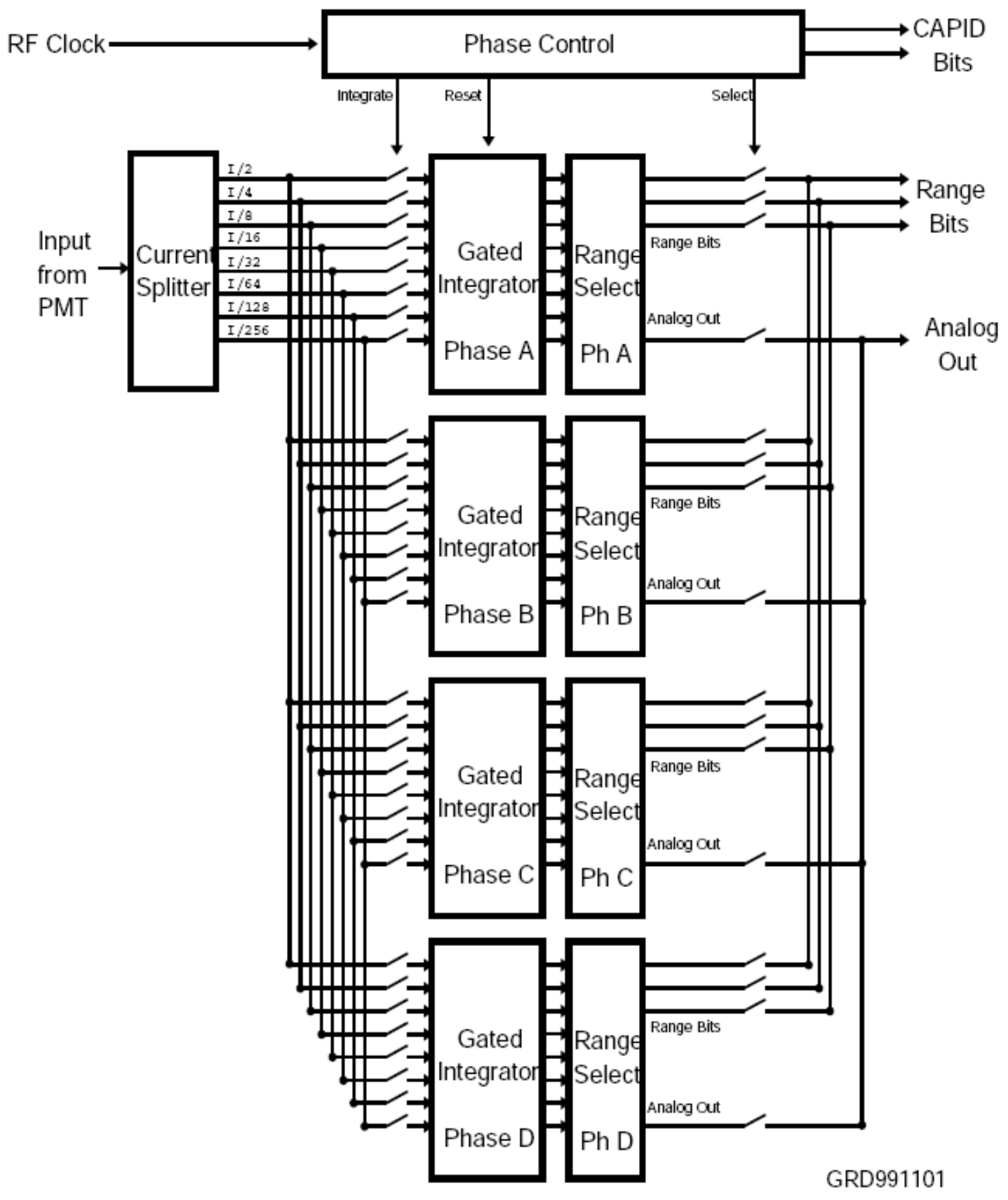

Figure 2.15: Block diagram of a QIE chip. It has three main components, current splitter, integrator and a range selector. The chip digitizes the analogue signal from the PMTs. 


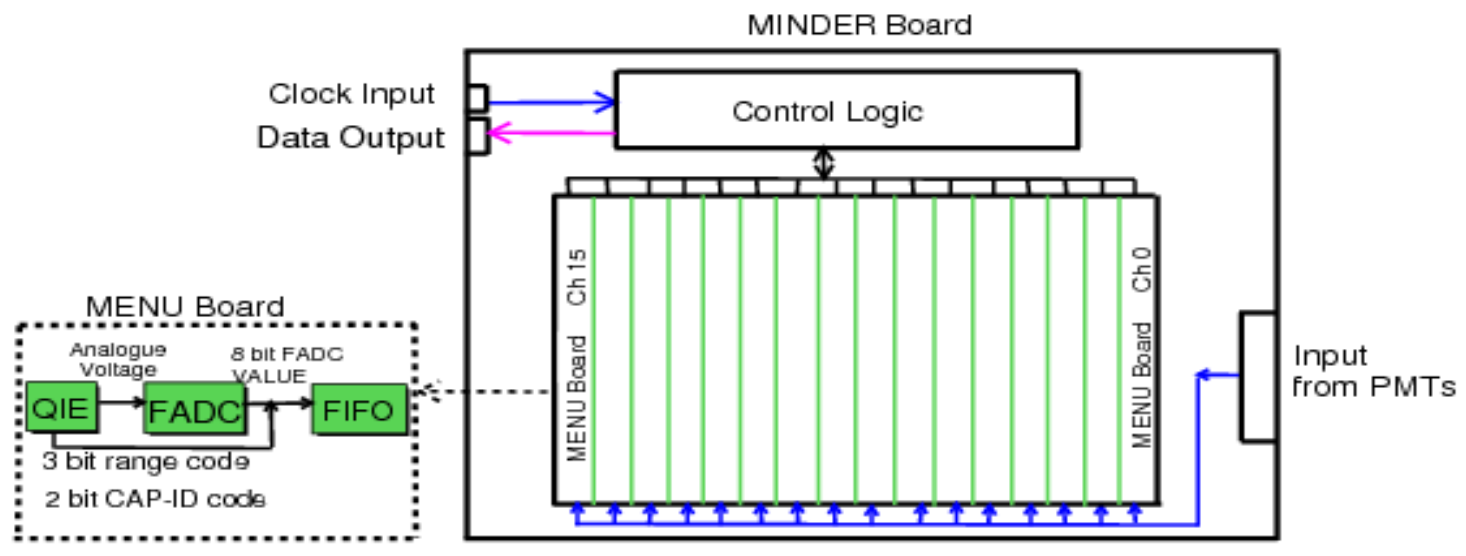

Figure 2.16: A MENU Board(a) and a MINDER Board(b).

MENUs. Signals from the PMTs are sent to the MENUs via the inputs on the MINDERs. Fig. 2.16 shows a MENU board and a MINDER. Eight MINDERS are connected to a VME board called the MASTER (MINOS Acquisition, Sparsifier and Time Stamper for Event Records). There are eight MASTERS in all that receive and process digitizations from their MINDERS but digitizations below a certain threshold(0.3 p.e.) are rejected.

\subsubsection{Data Acquisition}

The data from the front-end electronis(MASTERs) is stored in an array of trigger processors by the DAQ. A schematic diagram of the DAQ is shown in Fig. 2.17. As mentioned earlier, the front end electronics writes the data to a pair of buffers. The buffers are read alternatively by the Read Out Processors(ROPs) for one "timeblock"(10-50 ms long). The ROPs send the collected data to the Branch Readout Processor(BRP) after one "timeframe"(1 second long). The BRP collects all the data from the connected ROPs and transfers it to the trigger farm.

The trigger farm uses temporal and spatial information of scintillator hits to form "events". Information about the calibration of PMT channels is also stored by the trigger farm. Details of the calibration are given in chapter 3 . Events that pass the triggering algorithm are stored in the output 


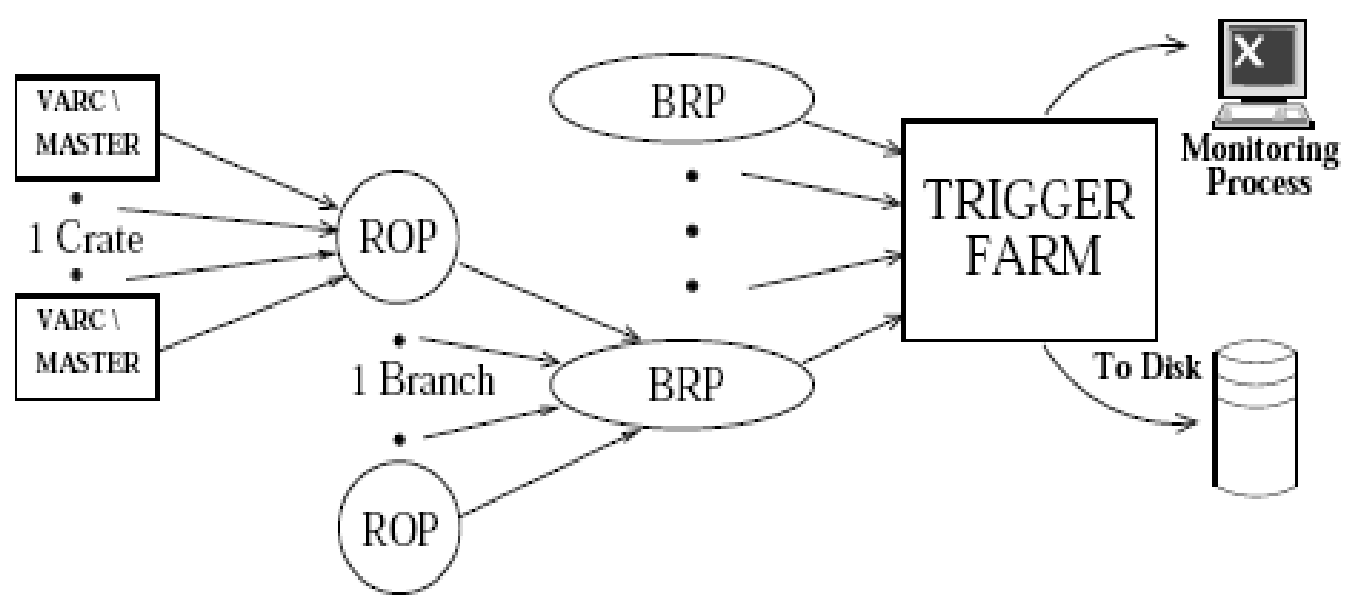

Figure 2.17: Schematic diagram of the MINOS Detector DAQ layout[43].

disk.

\subsection{FAR DETECTOR}

The Far Detector(FD) shown in Fig. 2.18 is an octagon which has a diameter of 8m, length of $31 \mathrm{~m}$ and a mass of 5.4 kton. The FD has two super modules and each module has 243 steel planes. There is a toroidal magnetic field of average strength $1.5 \mathrm{~T}$ and the magnetic coil hole is located at the center of the detector. The basic design of the detectors are similar but there are important differences between the two detectors.

One of the main differences is that the neutrino event rate at the FD is significantly lower (by a factor of $10^{6}$ per unit mass) than that at the ND. So the FD has a larger size in order to detect as many neutrinos as possible. It is placed $2341 \mathrm{ft}$ underground to reduce the cosmic ray background compared to the neutrino interaction rate. The instrumentation is uniform in the FD, where every 
steel plane is instrumented and a single plane has full coverage with 192 scintillator strips. The scintillator strips are readout at both ends, unlike the ND where the readout is single-ended. The WLS fibers carry the scintillation light to the M-16 Hamamatsu phototubes through clear optical fibers. Eight different fibers, from eight strips go into one PMT pixel(each PMT has 16 pixels) and are readout by one electronic channel. The 8-fold multiplexing is demultiplexed by the software using information about which fiber is connected to which pixel. Since the event rate is lower at the far detector, there are usually no overlapping events in a beam spill, so the electronic readout doesn't have to be as fast as the ND readout. 


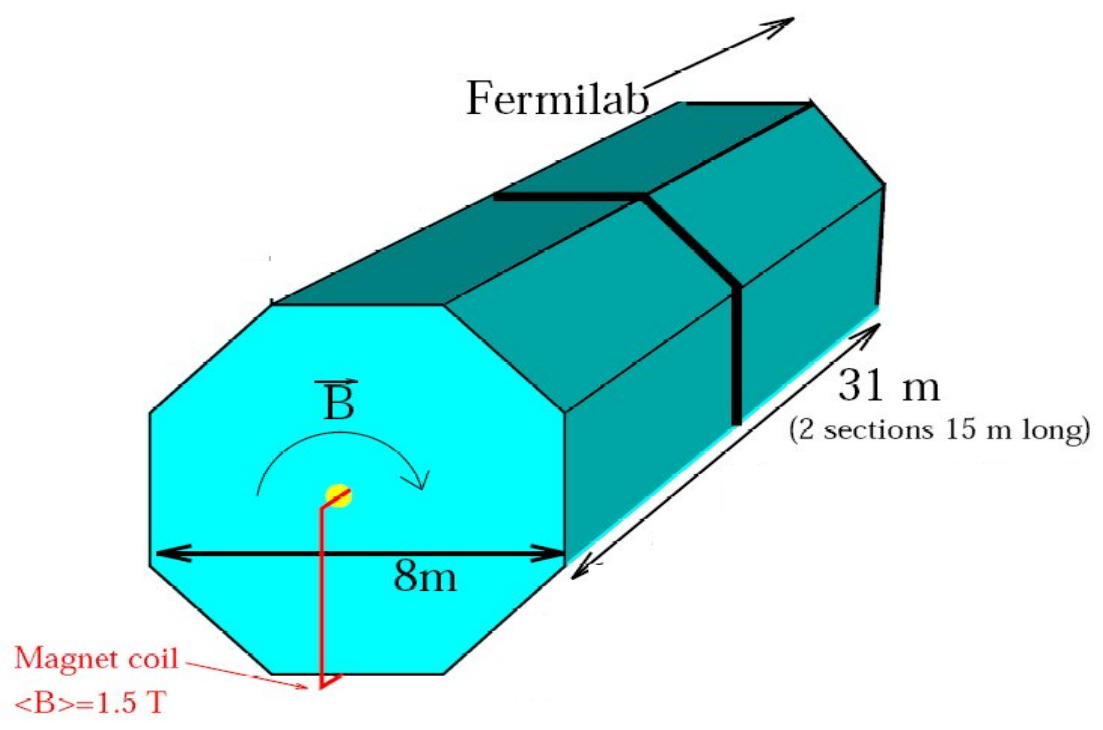

Figure 2.18: Sketch of the Far Detector located at Soudan Mine, Minnesota. Each plane has full coverage of scintillators. The coil hole is located at the center of the detector and is the origin of the co-ordinate system. 


\subsection{DETECTOR CALIBRATION}

MINOS measures the hadronic and electromagnetic shower energy by calorimetry. The shower energies are calculated from PMT signals (which are calibrated from integrated charge read out by electronics to energy). The spatial and temporal response of the PMT, scintillator and electronic outputs are calibrated using Light injection system and cosmic muons.

A calibration detector was installed at CERN for measuring MINOS response to dedicated $\mu, \pi, e$ and $p$ beams in the momentum range $0.2-10 \mathrm{GeV} / \mathrm{c}$. The first part of this chapter describes the calibration detector and the second part describes the calibration procedure. The last part discusses the uncertainties in the muon energy scale determination.

\subsection{CALIBRATION DETECTOR}

The calibration detector, or CALDET, was a scaled down version of the near detector. It had 60 planes that were $1 \mathrm{~m}$ wide. Each plane was instrumented with 24 vertical and 24 horizontal scintillator strips. It did not have a magnetic field because of its smaller size and the need for portability. The electronics and readout was similar to the near detector in order to effectively compare them. The detector collected data between 2001-2003.

The CERN PS(proton synchrotron) beam provided a mixture of hadrons, muons and electrons in the tunable momentum range of $0.2-10 \mathrm{GeV} / \mathrm{c}$ with momentum spread of approximately $1 \%$. CALDET collected data from two separate beamlines (T7 and T11) that used magnetic horns to focus the charged particles. Fig. 3.1 shows the measured fractional composition of the T7 beamline that was operated in positive and negative magnetic polarity. The electron contribution dominates the beam composition below $1 \mathrm{GeV} / \mathrm{c}$ and decreases exponentially with energy whereas the hadron 
contribution dominates above $3 \mathrm{GeV} / \mathrm{c}$.

The particles were identified using Cerenkov and Time of Flight(TOF) counters that were installed in the beamline. The beam particles have different masses which leads to different flight times which were measured by the TOF system. Charged particles emit Cerenkov radiation if they travel with a speed greater than the speed of light in that medium. The radiation is emitted at an angle that depends on the pressure of the medium $\left(\mathrm{CO}_{2}\right.$ in this case $)$ and mass and momentum of the charged particles.

The selection process used to differentiate between the particles depended on the beam momentum. The TOF system is used to identify $\pi \mu e$ and $p$ samples for beam momentum below 3 $\mathrm{GeV} / \mathrm{c}$. Gaussian fits were performed to determine the mean and the width of the TOF distributions. At all momentum values below $3 \mathrm{GeV} / \mathrm{c}$, the $\pi \mu e$ and $p$ mean TOF values were separated by more than $6 \sigma$. The Cerenkov counters were pressurized below the muon and pion threshold and were used to identify electrons. The electron contamination was less than $0.5 \%$. At higher beam momentum values $(>3 \mathrm{GeV} / \mathrm{c}$ ) the TOF data alone was not sufficient to separate pions and protons. To facilitate this separation data from two Cerenkov counters were used. The proton contamination in the pion beam(and vice verse) was negligible at all energies.

The Cerenkov counters and TOF system identified electron, proton and combined pion and muon components of the beam. Pion and muon separation was based on the topological difference between hadronic showers and muon tracks. Above $4 \mathrm{GeV} / \mathrm{c}$, most pions showered whereas most muons exited the detector. The samples were separated based on the distribution of average energy deposited per plane(higher for pions) and the total number of planes(larger for muons). For the lower momentum setting $(<4 \mathrm{GeV} / \mathrm{c})$ the separation is poorer. A discriminant was constructed from variables such as pulse height, number of scintillator strips and number of planes in an event. The measured fraction of muons in the $\mathrm{T} 7$ beamline at $1 \mathrm{GeV} / \mathrm{c}$ is $30 \%$, at $4 \mathrm{GeV} / \mathrm{c}$ is about $10 \%$ and decreases to $5 \%$ at $10 \mathrm{GeV} / \mathrm{c}[43]$. The muon contamination in the pion beam above $1 \mathrm{GeV} / \mathrm{c}$ is negligible, but is about $7 \%$ below this momentum. These separation techniques made it possible to run CALDET with relatively pure particle beams that were used to measure the detector response and to validate Monte Carlo simulations. 


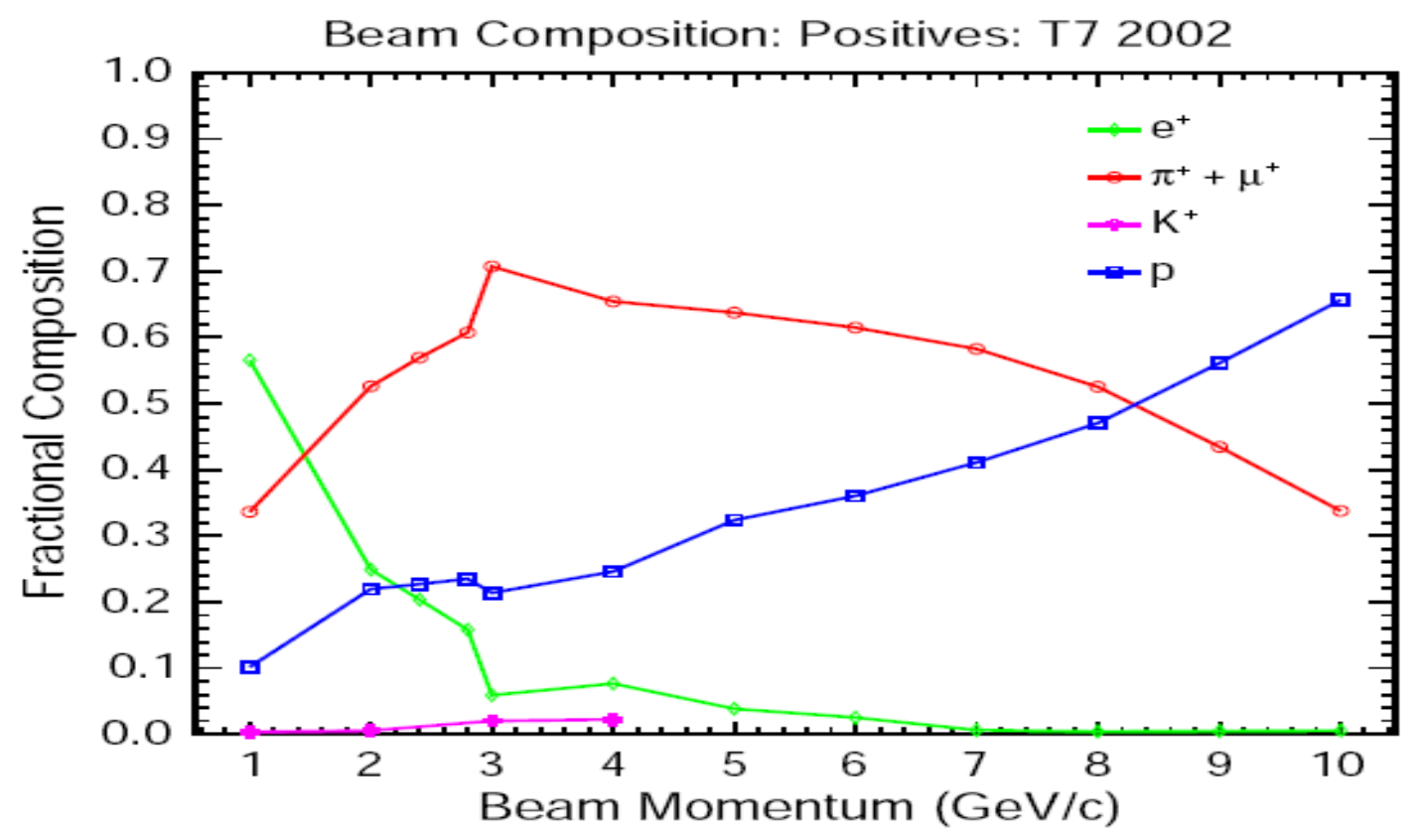

(a)

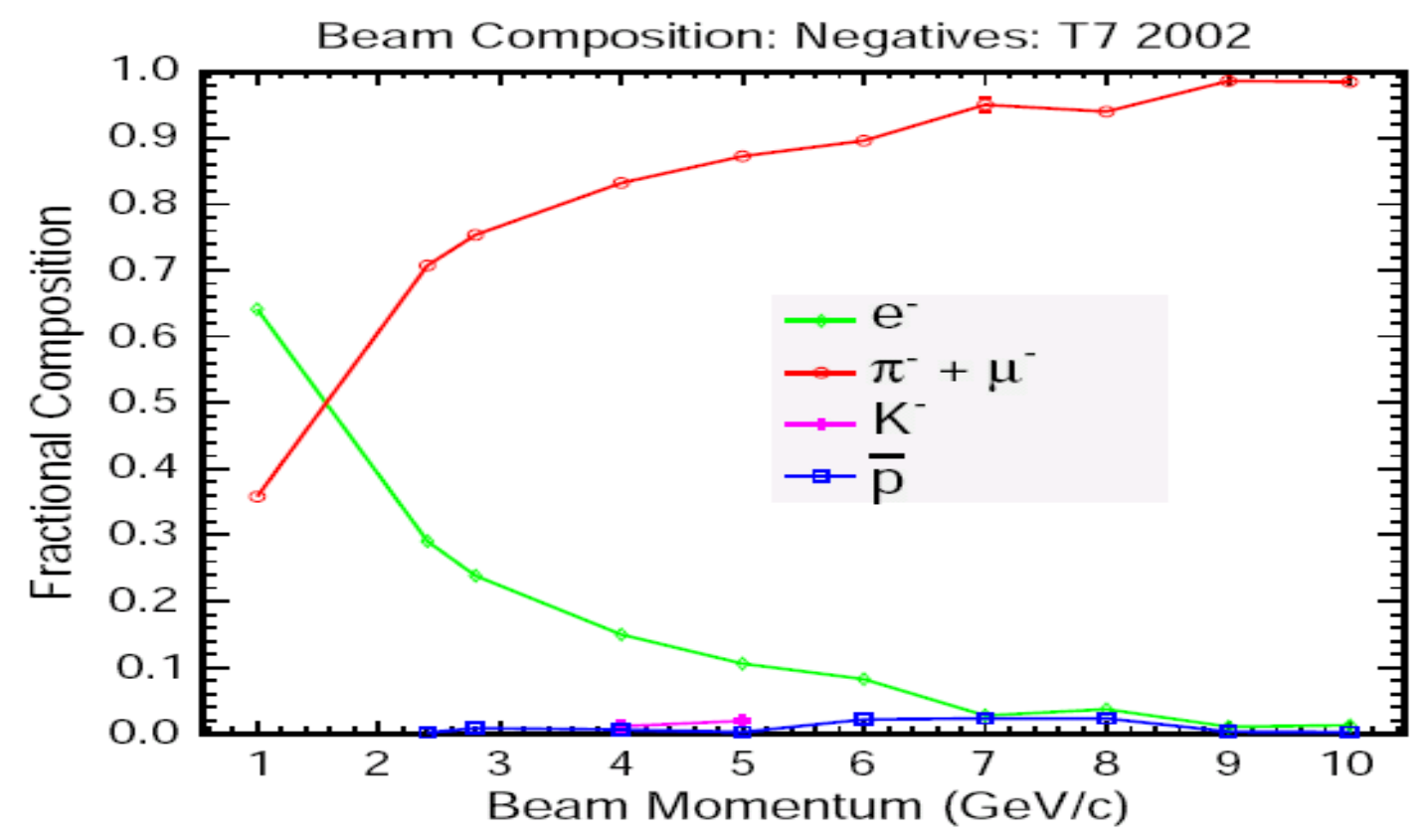

(b)

Figure 3.1: Measured fractional composition of the $\mathrm{T} 7$ beamline in positive and negative polarities[43]. 


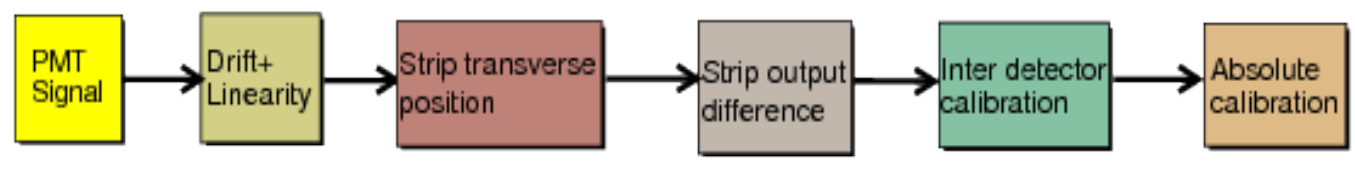

Figure 3.2: The MINOS data calibration chain.

\subsection{CALIBRATION PROCEDURE}

The steps followed in the calibration procedure can be summarized by the diagram shown in Fig. 3.2. The ADC output of the PMTs are corrected for drift and linearity. The corrected output is calibrated for variations in light output along the transverse position of the strip and also for strip-to-strip variations. The next step is to correct for detector-to-detector variation that converts the output to a standardized M.E.U.(Muon Equivalent Unit). The last step in the chain is to use different beams (electrons, muons, pions,protons) at the calibration detector to obtain the absolute energy scale. Except the last step, all other calibration steps are performed at the near detector and the calibration detector. The details of each step are given in the following sections.

\subsubsection{Gain drift and Linearity Correction}

The in-situ LED based Light Injection (LI) system pulses each strip periodically and corrects for gain (defined as the output from the PMTs in ADC count for a known level of input light in p.e) drift

in each PMT channel over a period of time and non-linearity in the gain for very high input $(\geq 100$ p.e.).

Each strip end is pulsed 1000 times/hour with 50 p.e. by the LI system to monitor the gain of each PMT channel. The gain is calculated daily from the LI data for all channels. The drift in gain that is caused due to seasonal variations and aging effects is less than $3 \%$ annually. The LI system can illuminate the PMT channels over a wide range of input light levels, this enables it to measure the non-linearity in the PMTs as well as the electronic channels that read out the PMTs. 


\subsubsection{Intrastrip Correction}

We need to correct for the variation in the strip response along the transverse length of the strip, which is mainly caused by the attenuation in the WLS fibers. The correction is given by

$$
A(x)=A_{1} e^{-x / L_{1}}+A_{2} e^{-x / L_{2}}
$$

where, $x$ is transverse position along the strip, $L_{1}$ and $L_{2}$ are the two attenuation lengths of the green light (for two wavelengths). The module mapper measured the response of each scintillator strip as a function of $x$ to a ${ }^{137} \mathrm{Cs}$ source. The calibration constants are later on checked using through going cosmic muons. The module mapper data is used to fit [15] for the parameters $A_{2} / A_{1}, L_{1}$ and $L_{2}$ in each strip.

\subsubsection{Strip-to-Strip Calibration}

The strip-to-strip calibration is performed using through-going cosmic ray muons that are suitable for this purpose because they are in the minimum ionizing region (few GeVs) and hence deposit an amount of energy that is relatively independent of their momentum. The cosmic ray muons correct for variations in the output from individual scintillator strips, differences in the attenuation length of the optical cables and differences in the quality of the optical connectors.

To characterize the response of an individual strip, the muon spectrum for that strip is constructed with cosmic ray muon data collected over a period of time. The strip-to-strip calibration constant, that is calculated once every month, is the strip response normalized by the mean response of all the strips.

\subsubsection{Muon Energy Equivalent(M.E.U.) Calibration}

M.E.U. is defined as the detector response to a perpendicular minimum ionizing particle(with an approximate energy of $0.5-1.1 \mathrm{GeV}$ ) traversing 1 plane of scintillator. This calibration, that converts the corrected ADC value to M.E.U., is necessary to compare the response of the ND with the CALDET. Stopping muons are used for this purpose because their energy can be more accurately reconstructed than exiting muons. 
To reduce the dependence of the relative calibration on the reconstruction uncertainty at the detectors, the first $90 \%$ of a muon track is chosen where the $\mathrm{dE} / \mathrm{dx}$ changes slowly $(\sim 8 \%)$ and the muon has an energy between $0.5-1.1 \mathrm{GeV}$. The later $10 \%$ of the track where the muon rapidly loses energy due to ionization is discarded. M.E.U. is defined as

$$
M E U=M e d i a n\left(\left(\frac{1}{N_{P}} \sum_{i=1}^{N_{P}} \frac{S_{i}}{L_{i}}\right)_{1} \ldots \ldots\left(\frac{1}{N_{P}} \sum_{i=1}^{N_{P}} \frac{S_{i}}{L_{i}}\right)_{N}\right)
$$

where, $N$ is the number of stopping muons in the sample,

$N_{p}$ is the number of planes in the portion of the track where $\mathrm{dE} / \mathrm{dx}$ changes slowly,

$S_{i}$ is the detector response in SigMap units in plane $i$, and $L_{i}$ is the perpendicular path length in plane $i$.

\subsubsection{Absolute Energy Scale}

The calibration detector described in section 3.1 is used to obtain the absolute energy scale by an energy/particle dependent M.E.U. to GeV conversion factor. Fig. 3.3 shows the CALDET response to a beam of hadrons and electrons. Here the detector response is expressed in terms of MIPs(minimum ionizing particle) which is equivalent to M.E.Us. At high energy (> $3 \mathrm{GeV})$, 60(47) MIPs are equal to $1 \mathrm{GeV}$ for hadrons(electrons).

The CALDET hadronic beam data is also used to tune the simulations for the relatively simple case of single particles with well known energy and identity. This is an important step because unless we are confident about the Monte Carlo simulations of single particles we cannot apply them to the neutrino interactions in which a mixture of hadronic particles will be produced in the final state. Several hadronic shower simulation codes(GFLUKA[33], GHEISHA[34], SLACGHEISHA[6], GCALOR[60]) were available but all these codes were for primary particles with energy above $10 \mathrm{GeV}$. Since MINOS hadrons have lower energy(0.5-10GeV), these simulation codes were compared to the output from the CALDET data to see which one best described the data. Data and Monte Carlo simulations of several variables were compared, such as number of hit strips, number of hit planes and calorimeter signal.

Fig. 3.4 shows the $\pi^{+}$calorimeter signal distribution for Monte Carlo simulation(GCALOR and SLAC-GHEISHA) and data at $1 \mathrm{GeV} / \mathrm{c}, 4 \mathrm{GeV} / \mathrm{c}$ and $8 \mathrm{GeV} / \mathrm{c}$. At $1 \mathrm{GeV} / \mathrm{c}$ and $4 \mathrm{GeV} / \mathrm{c}$, 


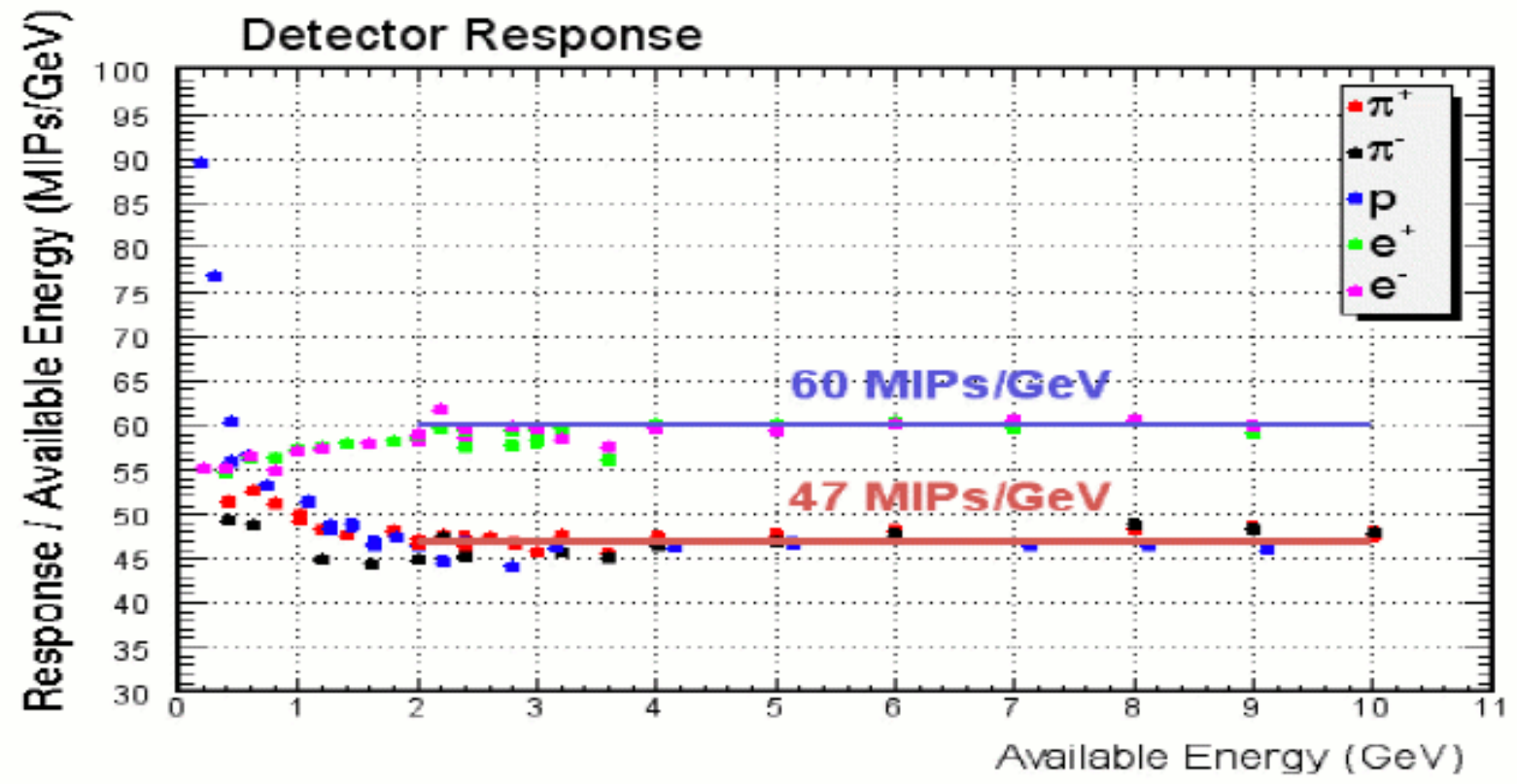

Figure 3.3: Detector response to hadrons and electron CALDET beams[43]. The unit MIP(minimum ionizing particle) is equivalent to MEU. The straight line shown in the plots is a fit to the high energy data. 
GCALOR(blue) describes the data better than GHEISHA(red). In general, at all momentum settings below $6 \mathrm{GeV} / \mathrm{c}$, GCALOR mean agrees with the data mean at the level of 2-3\% but has a slightly larger width. At $8 \mathrm{GeV} / \mathrm{c}$ (and higher momentum settings) none of the simulation codes describe the data well, GCALOR overpredicts the response by about 5\% whereas GHEISHA underpredicts it.

Fig. 3.5 shows the proton calorimeter signal distribution for Monte Carlo simulation(GCALOR and SLAC-GHEISHA) and data at $1 \mathrm{GeV} / \mathrm{c}, 4 \mathrm{GeV} / \mathrm{c}$ and $8 \mathrm{GeV} / \mathrm{c}$. Agreement for proton data is generally poorer compared to the pion data. At $1 \mathrm{GeV} / \mathrm{c}$, both the simulation codes show poor agreement with the data. The discrepancy arises because low energy protons lose all their energy via ionization within the first few planes of the detector and are difficult to model. At $4 \mathrm{GeV} / \mathrm{c}$ (and all intermediate energies between $1 \mathrm{GeV} / \mathrm{c}$ and $6 \mathrm{GeV} / \mathrm{c}$ ), GCALOR code describes the data better than GHEISHA. At $8 \mathrm{GeV} / \mathrm{c}$ (and higher momentum settings), the proton data and Monte Carlo comparison has a similar trend as seen in the pion distribution, with the GCALOR mean higher than the data mean by about $5 \%$.

The GCALOR code was found to predict the hadronic energy spectrums better than the other simulation codes below $6 \mathrm{GeV} / \mathrm{c}$. Above this momentum setting, we take a conservative estimate of the data and MC disagreement to be 5\% that is determined from the difference in the mean of the GCALOR simulation and data distribution of the calorimeter signal.

The major source of uncertainty on the hadronic energy scale is the 5\% data Monte Carlo simulation discrepancy. Uncertainty in beam energy at CALDET contributes 2\%. The intra-detector calibration with cosmic ray muons contribute $1.7 \%$. The M.E.U. at CALDET was determined from beam muons as well as cosmic muons, the difference in the value from these two procedures is $1 \%$. Adding all these sources of uncertainty in quadrature gives a total uncertainty of $5.6 \%$ on the knowledge of the hadronic scale.

\subsection{MUON ENERGY SCALE}

The muons produced in a charged current interaction can either stop in or exit the detector. A contained muon primarily loses energy by ionization. The energy loss per unit length is given by the 


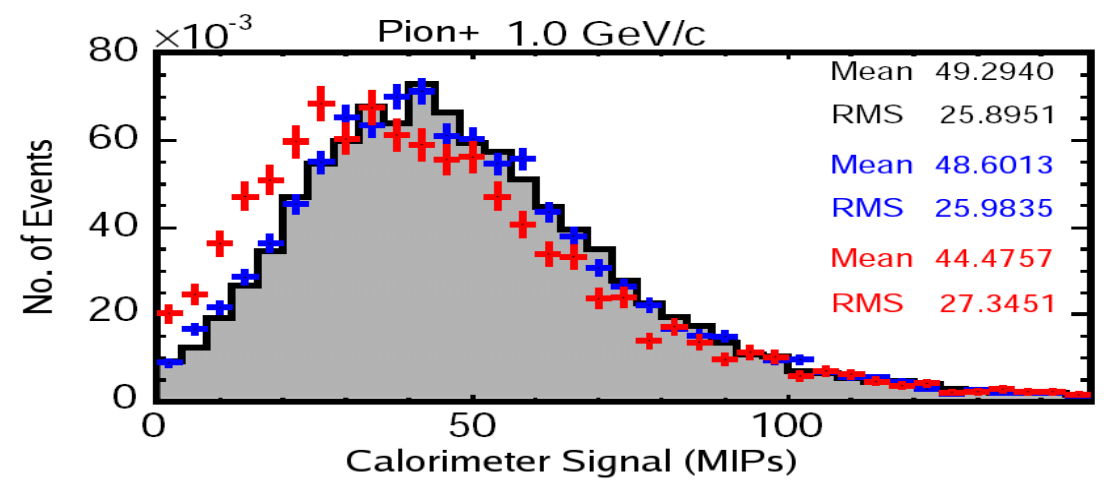

(a)

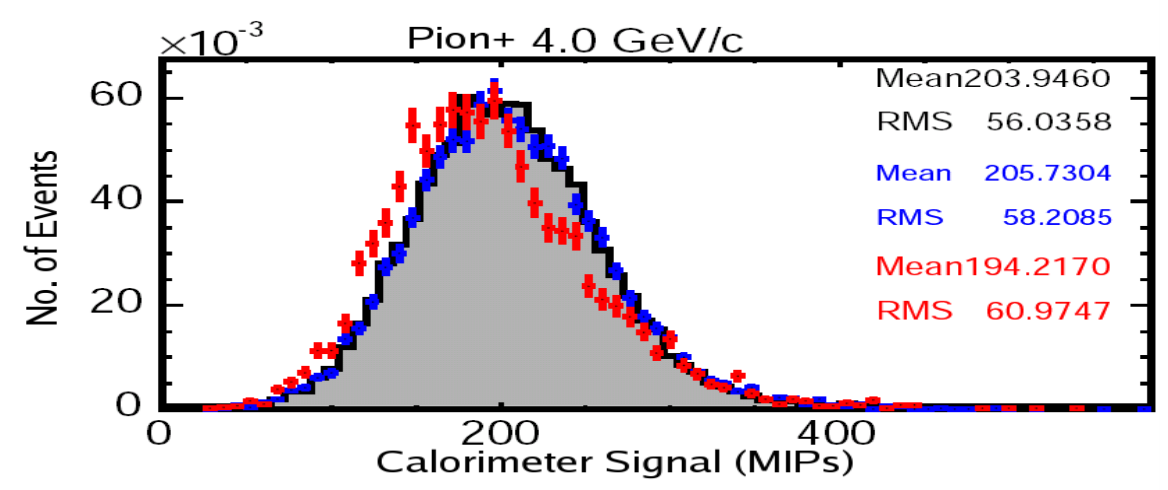

(b)

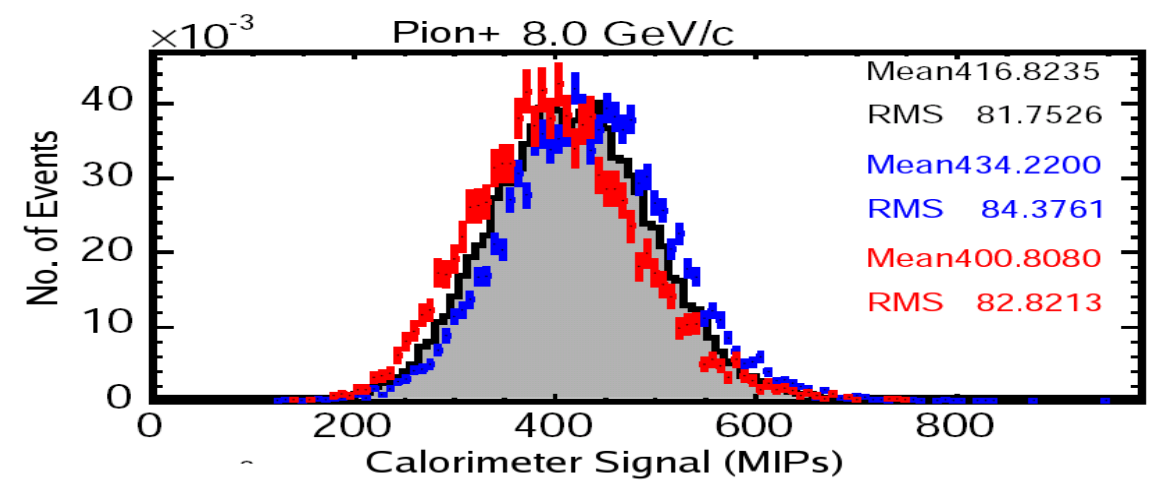

(c)

Figure 3.4: $\pi^{+}$calorimeter signal distribution(MIPs) at momentum settings of $1 \mathrm{GeV} / \mathrm{c}, 4 \mathrm{GeV} / \mathrm{c}$ and $8 \mathrm{GeV} / \mathrm{c}$. Data is shown with the shaded histogram, GCALOR simulation is shown in blue and SLAC-GHEISHA simulation is shown in red. The histograms are normalized to unit area. 


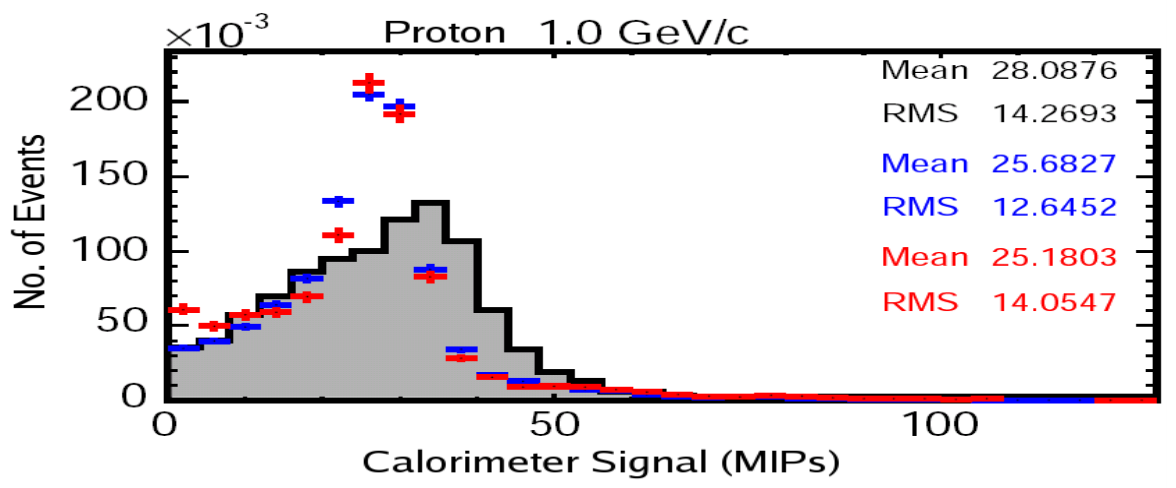

(a)

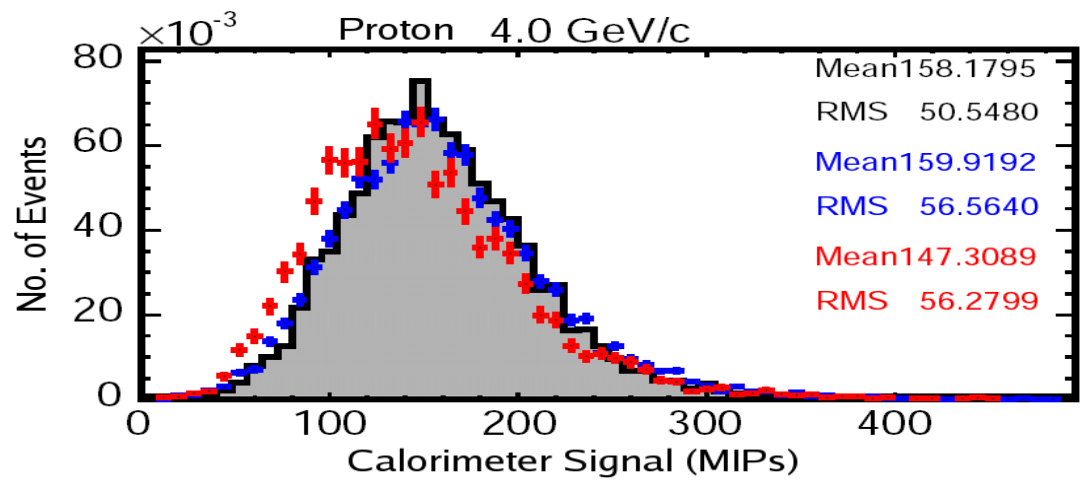

(b)

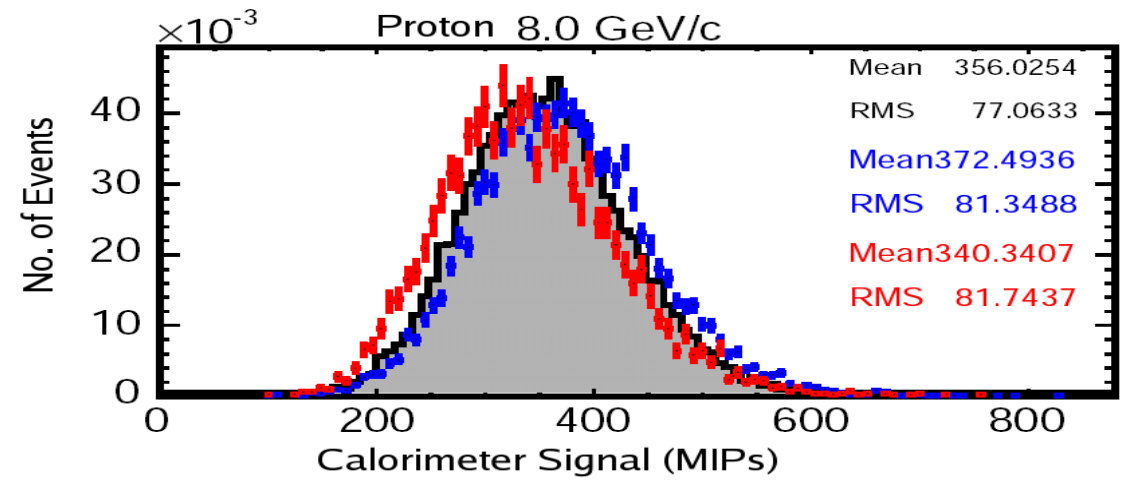

(c)

Figure 3.5: Proton calorimeter signal distribution(MIPs) at momentum settings of $1 \mathrm{GeV} / \mathrm{c}, 4$ $\mathrm{GeV} / \mathrm{c}$ and $8 \mathrm{GeV} / \mathrm{c}$. Data is shown with the shaded histogram, GCALOR simulation is shown in blue and SLAC-GHEISHA simulation is shown in red. The histograms are normalized to unit area. 
Bethe-Bloche equation [19]. The momentum is determined by calculating the length traversed in each material and using Groom's table[41] that is a parametrized version of Bethe Bloche equation. Details of length and momentum reconstruction are given in Chapter 4.

The precision on the range momentum scale is estimated using a Monte Carlo event sample with contained tracks. The reconstructed and true muon energy for these tracks disagree at the $1 \%$ level above $1 \mathrm{GeV}$. This disagreement is considered as an uncertainty. The energy loss in the simulation is compared to its value given in Groom's muon stopping power table [41] and they agree to $1 \%$. The detector mass model that holds information about the thickness and position of the steel/scintillator planes and the coil hole accounted for the relevant energy losses to within $1 \%$. These effects give an uncertainty of about $2 \%$ on the range momentum.

For an exiting track the momentum is measured from its curvature in the magnetic field. The uncertainty on the measurement of momentum from curvature is estimated by comparing the ratio of range momentum to curvature momentum for contained tracks between data events and simulated events. The difference between the Gaussian mean for data and Monte Carlo is $1.3 \%$ for contained tracks. We need to correct this number for exiting tracks that spend less time in the detector. The measured momentum from curvature for these tracks scales with the magnetic field $\Delta p \propto \Delta B$. The corresponding error on the magnetic field can be calculated by using a simplified $\operatorname{model}[50]^{1}$.

Let us assume that a track with initial true momentum $p_{0}$ traverses $N$ planes and loses momentum $\Delta p$ in each plane. The momentum from curvature $\left(P_{v t x}\right)$ of a stopping track after it has travelled $n$ planes can be written as

$$
P_{v t x}=\left(p_{o}-n \Delta p\right)+n \Delta p
$$

The quantity $\left(p_{0}-n \Delta p\right)$ is measured by the magnetic field. The final momentum measured from curvature will be an average over the $N$ planes

$$
<P_{v t x}>=\frac{\sum_{n=1}^{N}\left[\left(p_{o}-n \Delta p\right)+n \Delta p\right]}{N}=p_{0}
$$

If the magnetic field is mis-measured by a factor $x$, then the modified momentum will be

\footnotetext{
${ }^{1}$ Personal communication with Justin Evans
} 


$$
\begin{aligned}
<P_{v t x}^{\text {mod }}> & =\frac{\sum_{n=1}^{N}\left[x\left(p_{o}-n \Delta p\right)+n \Delta p\right]}{N} \\
& =\frac{N x p_{0}+\left[\sum_{n=1}^{N} n\right][\Delta p][1-x]}{N} \\
& =x p_{0}+\frac{1}{2}(N+1) \Delta p(1-x) \\
& =x p_{0}+\frac{1}{2}\left(p_{0}+\Delta p\right)(1-x) .
\end{aligned}
$$

Here $N \Delta p=p_{0}$ for stopping tracks. For a 10 plane track, $\Delta p=p_{0} / 10$ and Eq. 3.6 can be written as

$$
<P_{v t x}^{m o d}>=\frac{1}{20} p_{0}[9 x+11]
$$

The fractional change in the measured momentum is

$$
\frac{\delta p}{p_{0}}=\frac{<P_{v t x}^{m o d}>-<P_{v t x}>}{p_{0}}=\frac{9}{20}(1-x)
$$

We have estimated fractional uncertainty in momentum $\delta p / p_{0}$ to be $1.3 \%$, then the corresponding change in the magnetic field is $\frac{20}{9} \times 1.3 \%=2.2 \times 1.3 \% \approx 3 \%$. If the number of planes increase in the track $\Delta p / p \rightarrow 0$ and the change in magnetic field $\sim 2 \times 1.3 \%=2.6 \%$.

We take a conservative estimate of uncertainty on the momentum measured from curvature to be $3 \%$ (w.r.t. range momentum). This is obtained by multiplying the $1.3 \%$ error on the data and MC disagreement of contained tracks with a factor of 2.2 that corrects for the effect of magnetic field. The final $4 \%$ uncertainty on the curvature momentum is obtained by quadratically adding this $3 \%$ difference and the absolute $2 \%$ uncertainty on range momentum. 


\subsection{RECONSTRUCTION}

Neutrino interactions are reconstructed using energy deposition, timing and topology of the scintillator strips that have been "hit" by traversing particles. Since a typical $10 \mu$ s spill contains overlapping events, a spill is "sliced" into individual candidate events based on the spatial and timing information of the hits. Tracks and showers are constructed from the slices which are then put together to form events. Several properties(vertex, length, angle, energy etc.) are assigned to the reconstructed tracks, showers and events. Fig. 4.1 shows the event display of a reconstructed muon neutrino charged current interaction. The signature of a charged current event is a well defined muon track with hadronic activity at the vertex. Fig. 4.2 shows the steps followed in the standard reconstruction procedure. Details of each step are given below.

\subsubsection{Hit Strip formation}

The PMTs are readout once every 19ns. A hit strip signal spans more than one readout cycle and gets divided into two or three timing buckets (where each bucket is $19 \mathrm{~ns}$ long). The ADC value in each bucket is referred to as one digit. The total charge in the PMT pulse is proportional to the sum of all the digits. The hit strips are formed by associating digits that have come from the same strip, have a time difference of less than 60 ns between them and a total time span of less than 120 ns.

\subsubsection{Slice formation}

Slices are defined[51] based on the timing and proximity of hit strips and are likely to contain individual events. Initially, calorimeter strips with a charge greater than 2 p.e, a time gap(between two strips) of less than $20 \mathrm{~ns}$ and a total time span of less than $300 \mathrm{~ns}$ are formed into a slice. A slice 


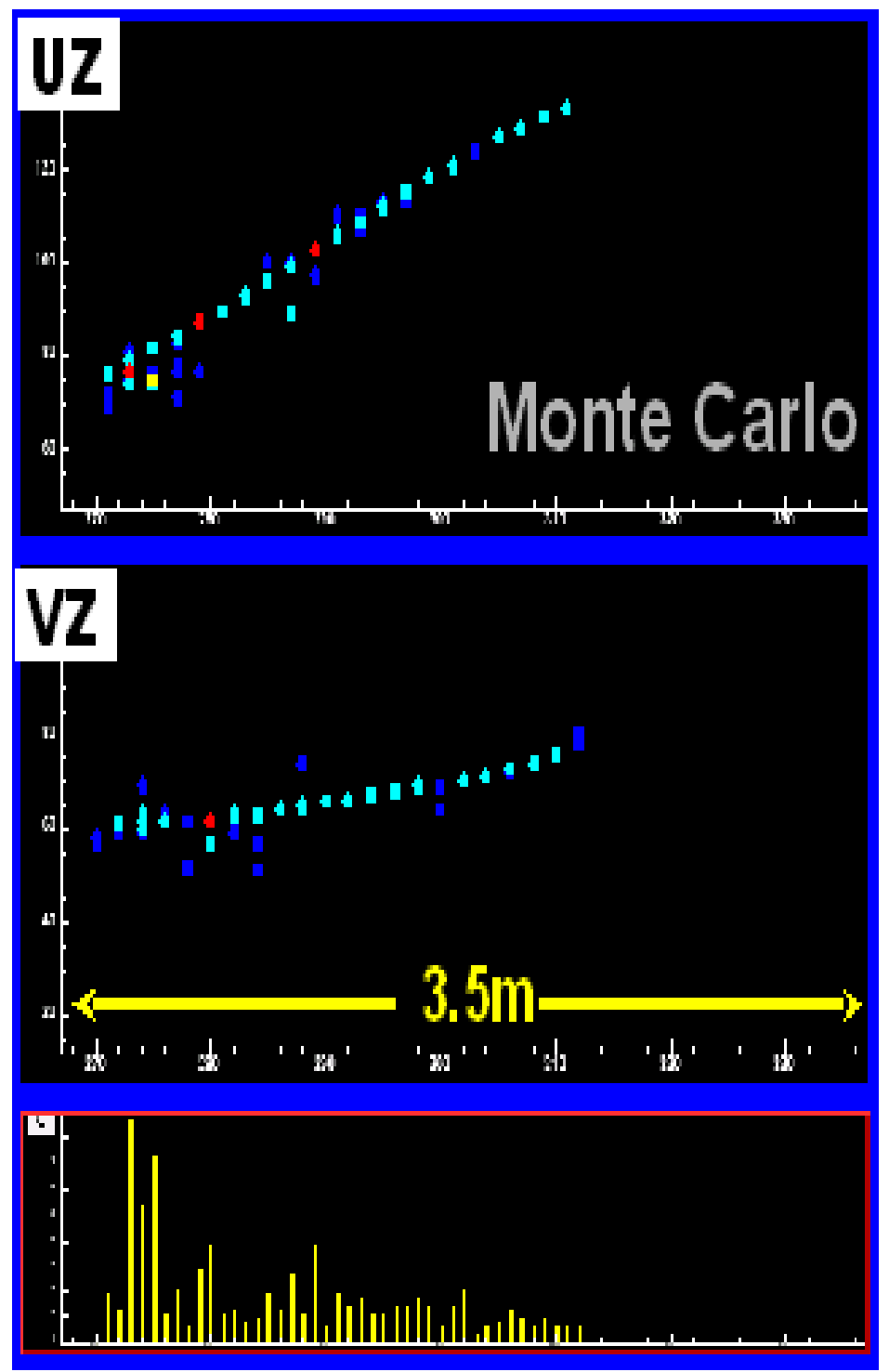

Figure 4.1: Muon neutrino charged current interaction simulated in the MINOS detector. The U versus $\mathrm{Z}$ and the $\mathrm{V}$ versus $\mathrm{Z}$ view are shown at the top and the bottom respectively. The colored boxes show the energy deposited in each scintillator strip. The last plot shows the pulse height distribution in each strip as a function of longitudinal distance. The energy deposited towards the beginning of the track is the largest and gradually decreases with distance. 


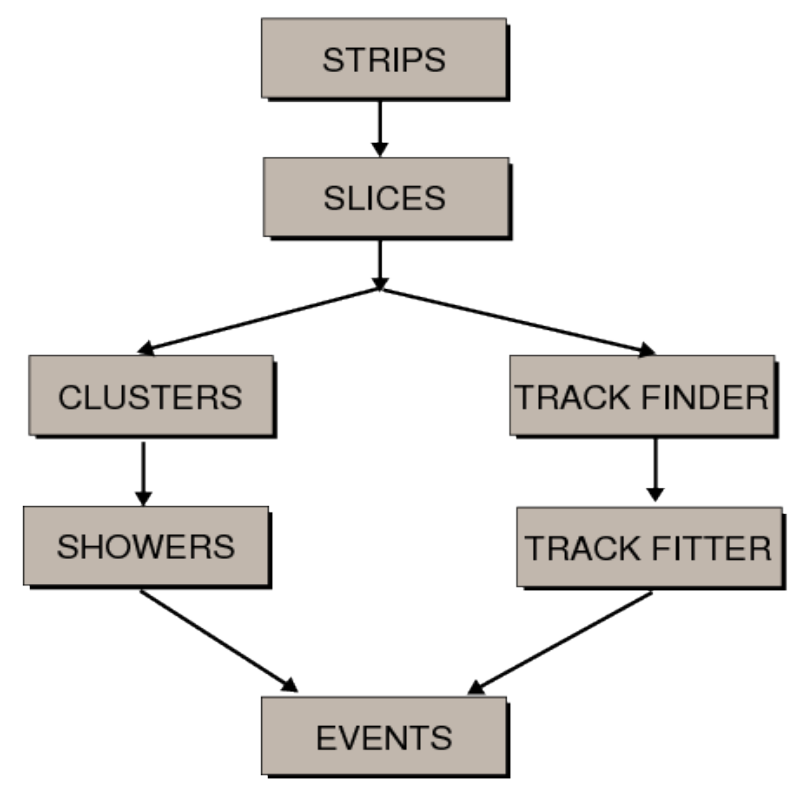

Figure 4.2: Standard reconstruction chain in MINOS.

must have at least two strips. If the strips in a slice have a gap of more than $1 \mathrm{~m}$ in the longitudinal direction then the slice is split into two provided the slice does not span the coil hole.

Spectrometer strips and calorimeter strips that have a charge smaller than 2 p.e are added to the most appropriate slice based on timing information. Finally any slice with a total charge of less than $2000 \mathrm{ADC}(\sim 200 \mathrm{MeV})$ counts is discarded. Fig. 4.3 shows a slice formed from strip hits in the ND.

\subsubsection{Track formation}

Clusters are formed from each slice by grouping together calorimeter hits that lie in the same plane, have a minimum charge of 2 p.e. and are separated by less than two strip widths $(4.1 \mathrm{~cm})$. Once a cluster is formed, a flag is set that determines if a plane in the cluster is more likely to belong to a track or to a shower. If the neighboring clusters are densely packed together then the plane is tagged to be a "shower-like" plane otherwise it is a "track-like" plane.

Triplets are formed from small track segments in each view from group of three clusters as 


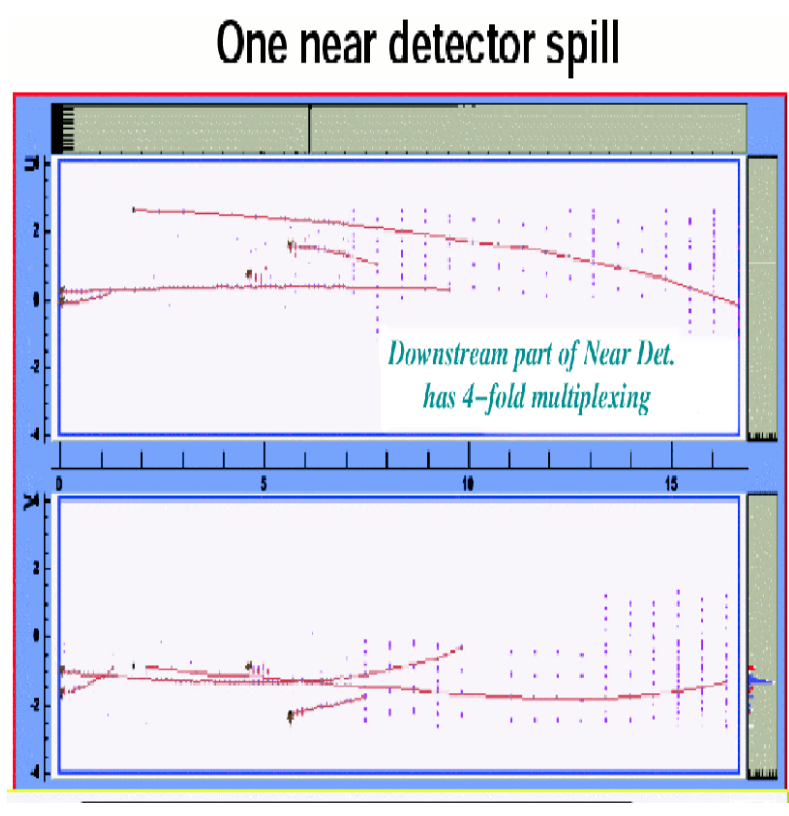

(a)

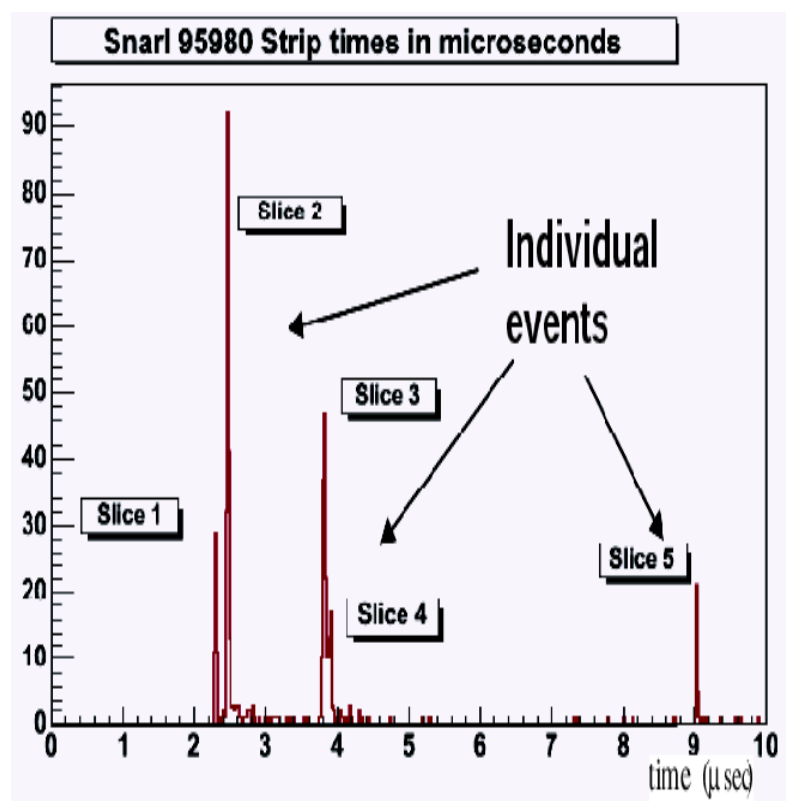

(b)

Figure 4.3: Figure on the left shows the hits in a Near Detector spill in the $U$ versus $Z$ view in the top and $\mathrm{V}$ versus $\mathrm{Z}$ view in the bottom. A spill with relatively few events has been chosen intentionally for clarity. Figure on the right shows the strips separated into slices based on timing and topology of hits. The Y-axis shows the number of strips and the $\mathrm{X}$-axis shows the time in $\mu$ s. 
shown in Fig. 4.4. If a series of triplets are found that have overlapping clusters then they are joined together to form a 2 dimensional(2D) track segment in each view. Once a complete list of $2 \mathrm{D}$ tracks in each view has been determined, they are matched between the two views by searching for overlapping planes. The criterion for matching track segments is that the difference between the beginning plane(or the end plane) for the two views must be less than 10 and the difference between the beginning plane in one view and the end plane in the other view should be larger than 2.

After matching the two views, track finding is basically complete but the clusters in the track may contain multiple hits (like in a vertex shower) and the next step is to choose the most suitable hit that belongs to the track. To select the most suitable hit from a cluster, a linear fit is performed in each view through the "track-like" planes. The hit (in the "shower-like" plane) that is intercepted by (or is closest to) the straight line is then included in the track.

Once a track is reconstructed, some initial properties of the track are determined. The $U$ and $\mathrm{V}$ position of each hit(by interpolation), length of the track, momentum from range, the track beginning and end are assigned to each track. The angle w.r.t the $\mathrm{z}$ direction, $d u / d z$ and $d v / d z$ are calculated by fitting a straight line to the first three hits in the track in the $\mathrm{U}$ and $\mathrm{V}$ view.

The spectrometer region of the ND has four-fold electronic multiplexing that is de-multiplexed in the track fitting stage. Track formation package makes no attempt to pick up any spectrometer hit. All the properties assigned to the track at this stage are preliminary, they are refined later after fitting the track.

\subsubsection{Track Fitting}

The track fitting is performed by using a Kalman filter (reference Appendix A) which iteratively solves a set of simple equations to minimize the mean of squared error. The filter provides a complete description of a muon track at any given position in the detector. Thus the output of the filter can be used to refine our track finding, especially if the vertex of the track is embedded in a large shower.

We define a $5 \times 1$ dimensional state vector ( $\mathrm{u}$ and $\mathrm{v}$ position, $\mathrm{du} / \mathrm{dz}, \mathrm{dv} / \mathrm{dz}$ and $\mathrm{q} / \mathrm{p}$ (ratio of the charge to momentum measured from curvature)) for each candidate track. The initial estimate of 


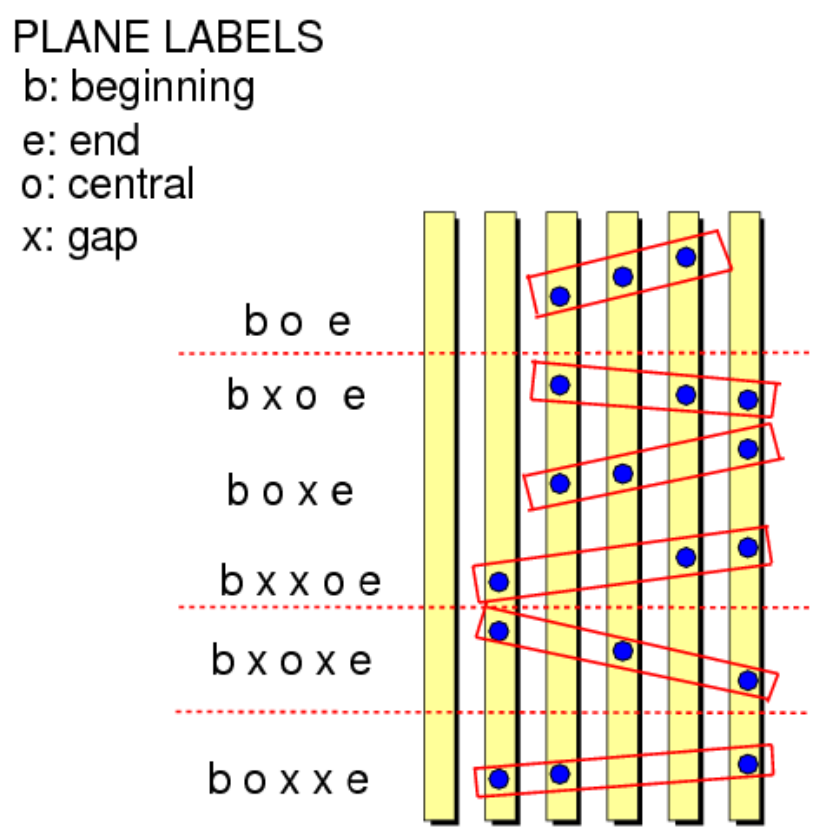

Figure 4.4: The various ways in which a triplet can be formed at the Near Detector[48]. The clusters must be in the same view. The blue circles represent a cluster and can contain more than one hit. 
this vector is taken from the first plane in the track where the $\mathrm{u}, \mathrm{v}, \mathrm{du} / \mathrm{dz}, \mathrm{dv} / \mathrm{dz}$ values are the initial values from the track formation package and the $q / p$ value is taken to be zero. The error covariance matrix is a $5 \times 5$ dimensional matrix and in an initial estimate each entry reflects the uncertainty in an element of the state vector.

A propagator matrix projects the state vector and the error covariance matrix from plane $k-1$ to plane $k$ by taking into account the effect of the magnetic and the energy loss due to multiple Coulomb scattering and ionization. The Kalman gain, that is calculated by minimizing the error covariance matrix, controls the influence of a single measurement on the final state vector. Once we have the Kalman gain, the state vector(also known as the Filtered State) and the error covariance matrix are updated. The procedure is repeated for the next plane by relabelling plane $k$ as plane $k$ - 1 . Convergence is obtained by moving from one plane to the next and back and forth along the entire track several times. With each iteration, a new set of strips are selected that most closely match the state vectors.

The spectrometer de-multiplexing is also performed by the track fitting package. The first spectrometer plane that contains a hit is selected and the state vector is projected to that plane. Out of the four possible hits, the one that matches the projected $\mathrm{u} / \mathrm{v}$ position (within three strip-widths) most closely is selected. The maximum allowed difference between the last calorimeter hit and the first spectrometer hit is 40 planes and that between any two planes in the spectrometer is 70 planes.

4.0.4.1 Setting Track Properties Once the complete track is determined, the track properties are set so that higher level reconstruction packages can use them. The beginning (end) $\mathrm{u}$ and $\mathrm{v}$ positions are the $\mathrm{u}$ and $\mathrm{v}$ position of the filtered state vector on the beginning(end) plane. The beginning (end) z position is the mid point of the scintillator plane that has registered the first(last) hit. The momentum from curvature is the $\mathrm{q} / \mathrm{p}$ value of the filtered state vector in the very first plane of the track. The sign of the $\mathrm{q} / \mathrm{p}$ value is taken to be the sign of the track charge. A $\mu^{-}\left(\mu^{+}\right)$track bends towards(away from) the $\mathrm{z}$-axis and has a negative(positive) sign. The $\chi^{2}$ value of the fit is the sum of the squared difference between the filtered transverse position and the actual transverse position of the hit. The angle is calculated from the $\mathrm{du} / \mathrm{dz}$ and $\mathrm{dv} / \mathrm{dz}$ value of the first hit plane in the track. From these two values we can calculate ds/dz, which is the inverse of the muon angle w.r.t. the $\mathrm{z}$ direction 


$$
\frac{d s}{d z}=\sqrt{1+\left(\frac{d u}{d z}\right)^{2}+\left(\frac{d v}{d z}\right)^{2}} .
$$

To obtain $\mathrm{du} / \mathrm{ds}(\mathrm{dv} / \mathrm{ds}), \mathrm{du} / \mathrm{dz}(\mathrm{dv} / \mathrm{dz})$ is multiplied with the reciprocal of Eq. 4.1 .

The momentum from range is calculated by summing the energy lost due to ionization in each segment(steel, scintillator or air) of the detector. To perform this summation the range(=length $\times$ density $\left.\left(\mathrm{g} / \mathrm{cm}^{2}\right)\right)$ is calculated for each material and the corresponding energy loss is determined from Groom's table [41].

\subsubsection{Cluster and Shower Formation}

The shower formation starts with cluster formation, which are conceptually similar to the clusters formed during track formation, but the criteria for grouping hits into a cluster are slightly different. For each hit strip, a neighbor map is constructed that has hits within $30 \mathrm{~ns}$ and less than 5 strip-widths from the hit in question. Clusters are formed in each view by grouping hits that are "neighbors" and are extended longitudinally by merging overlapping clusters.

The next step is to match the $2 \mathrm{D}$ showers in the two views to form $3 \mathrm{D}$ showers. It is required that the two clusters have a beginning plane within 10 planes and time within $30 \mathrm{~ns}$. The longitudinal overlap in the two views are required to be at least 50\%. In case the clusters are "short"(constitutes of less than 5 planes), these conditions are relaxed. The shower energy is constructed by summing the energy from each strip included in the shower. The cluster having the largest charge-weighted U/V position within 5 planes from the most upstream plane of the shower is selected as the vertex.

\subsubsection{Event Formation}

Event formation consists of associating tracks and showers in a given slice whose beginning vertices satisfy the following conditions; radial separation in the U-V plane less than $0.5 \mathrm{~m}$, longitudinal separation less than $1 \mathrm{~m}$ and the time separation less than $200 \mathrm{~ns}$. If these conditions are satisfied, then the track and shower likely originated from the same neutrino interaction. A post reconstruction condition is applied in which the shower and track vertex longitudinal separation is 
tightened to $0.5 \mathrm{~m}$. Once the event is formed, the shower energy is recalculated by excluding the energy depositions in the strips that belong to a track.

\subsection{RESOLUTION}

The momentum resolution is shown in Fig. 4.5 for tracks whose momentum is measured from range with true muon energy between 3 and $4 \mathrm{GeV}$. The resolution for the range momentum is about 5\% and mostly independent of the muon energy. The long tail on the right of the plot is from tracks that pick up extra hits and hence over estimate the track length. The shoulder on the left is caused by low purity tracks.

Fig. 4.6 shows the resolution for the reciprocal of the momentum for tracks whose momentum is measured from curvature with true muon energy between 12 and $13 \mathrm{GeV}$. The resolution is poorer, about $9 \%$ and is independent of muon energy. The gaussian shape of the resolution plot is from Multiple Coulomb Scattering. The tail on the right of Fig. 4.6 is from tracks that exit the detector from the side. These tracks have large angles that spend less time in the detector and have poor resolution. Tracks that end near the coil hole contribute to the shape on the left of the mean.

Fig. 4.7 shows the muon angle resolution as a function of true muon angle. The resolution degrades with increasing muon angle. As the muon angle increases, the time spent by the track in the detector decreases which makes the determination of muon angle less precise.

The shower energy resolution was determined by studies performed at the CALDET with separate pion, proton and electron beams. The energy deposited by a hadronic or electromagnetic shower in a calorimeter is proportional to $1 / \sqrt{E}$. The proportionality constant is different for the two types of showers and depends on the thickness of steel and scintillator planes. Fig. 4.8 shows the resolution for the two type of showers as a function of the available energy. The resolution of the electromagnetic shower is $23 \% / \sqrt{E}$ and hadronic shower is $55 \% / \sqrt{E}$. Hadronic showers have a widely fluctuating longitudinal and transverse shape whereas electromagnetic showers have a shape that fluctuates within narrow limits. In a neutrino $\mathrm{CC}$ interaction, the hadronic shower

energy will be carried by a mixture of final state particles such as $\pi^{+/-}, \pi^{0}$ (that form photons) and protons and the resolution will be different from that of single particles. The resolution of neutrino 

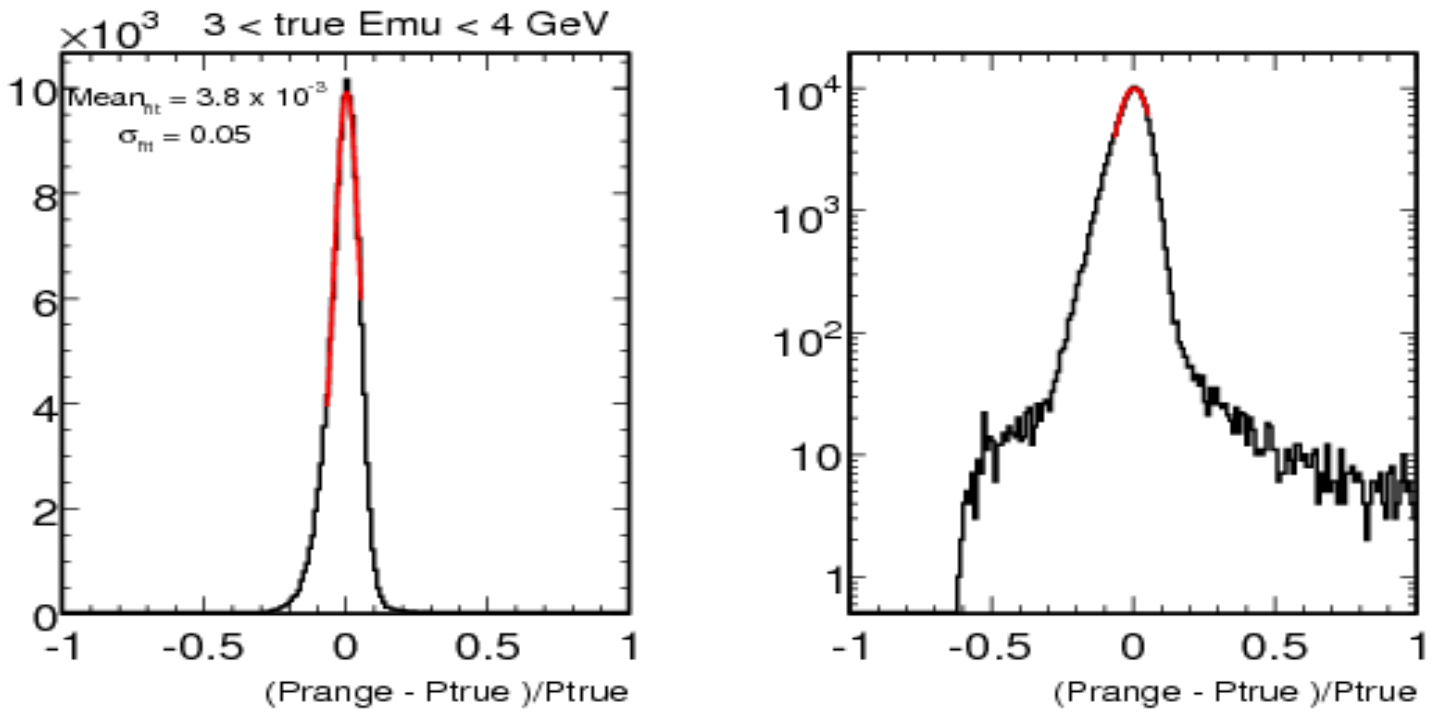

Figure 4.5: Resolution of momentum measured from range for tracks that stop in the detector with true muon energy between 3 and $4 \mathrm{GeV}$. The red line shows a Gaussian fit that has been performed between the two points at half maximum. The log version of the same plot is shown on the right. 

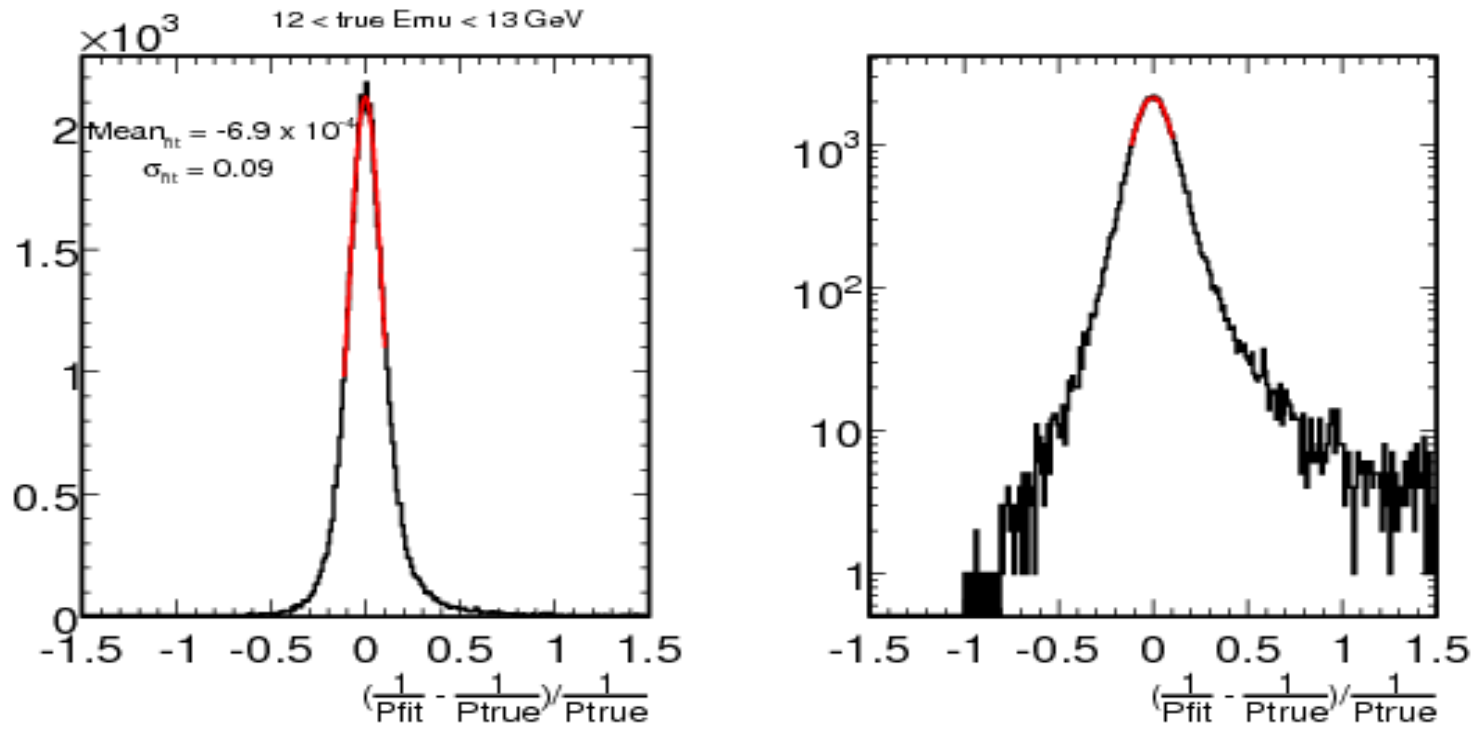

Figure 4.6: Resolution of reciprocal momentum measured from curvature for events having true energy between 12 and $13 \mathrm{GeV}$. The red line shows a Gaussian fit that has been performed between the two points at half maximum. The log version of the same plot is shown on the right. 


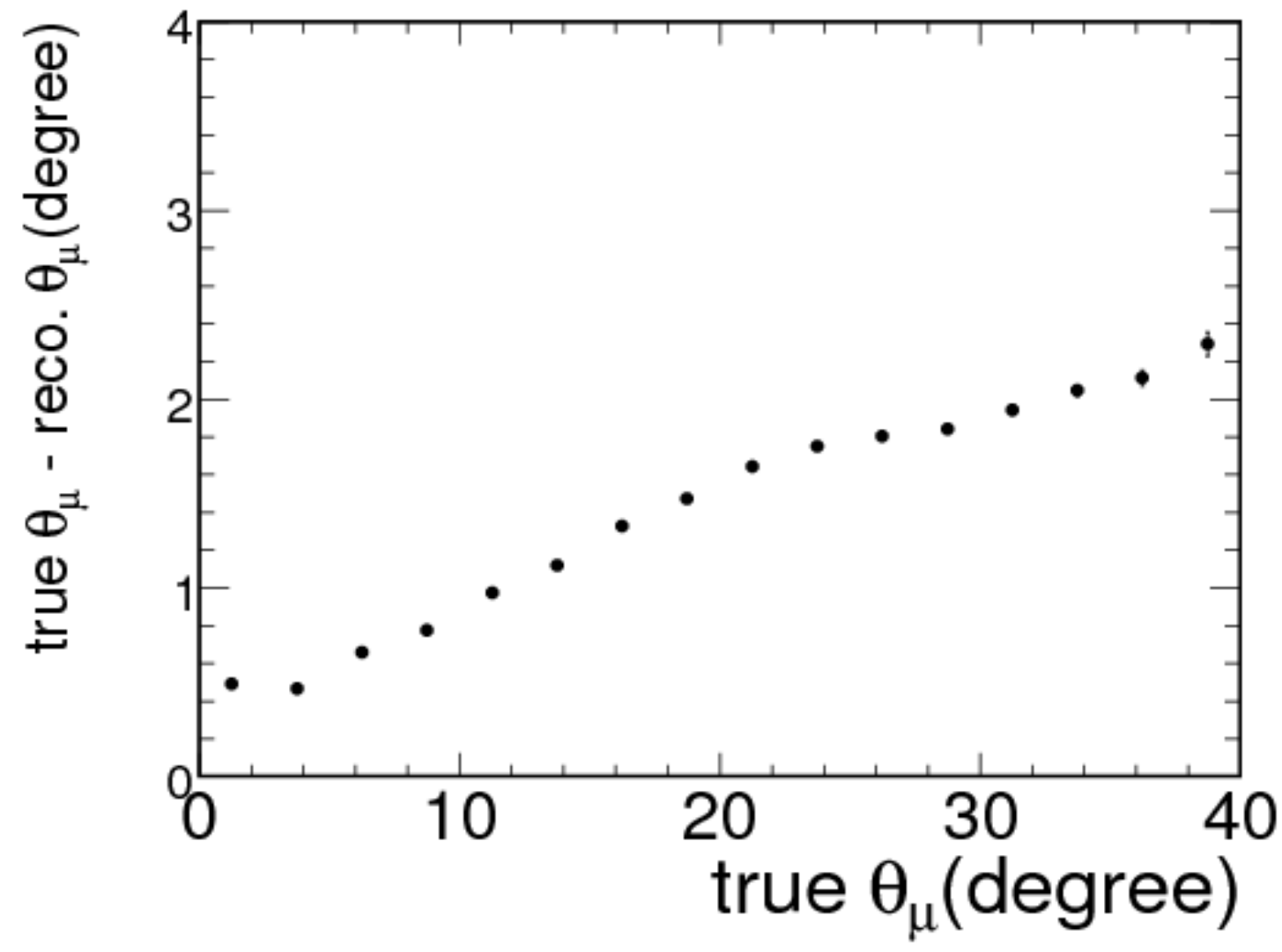

Figure 4.7: Muon angle resolution as a function of true muon angle. The resolution is plotted by fitting a gaussian to the distribution of (true $\theta_{\mu}$ - reconstructed $\theta_{\mu}$ ) in each true $\theta_{\mu}$ bin. 
induced hadronic showers that is determined from simulation based studies is about $59 \%$ at $1 \mathrm{GeV}$ and $32 \%$ at $3 \mathrm{GeV}[24]$. 


\section{Single particle energy resolution}

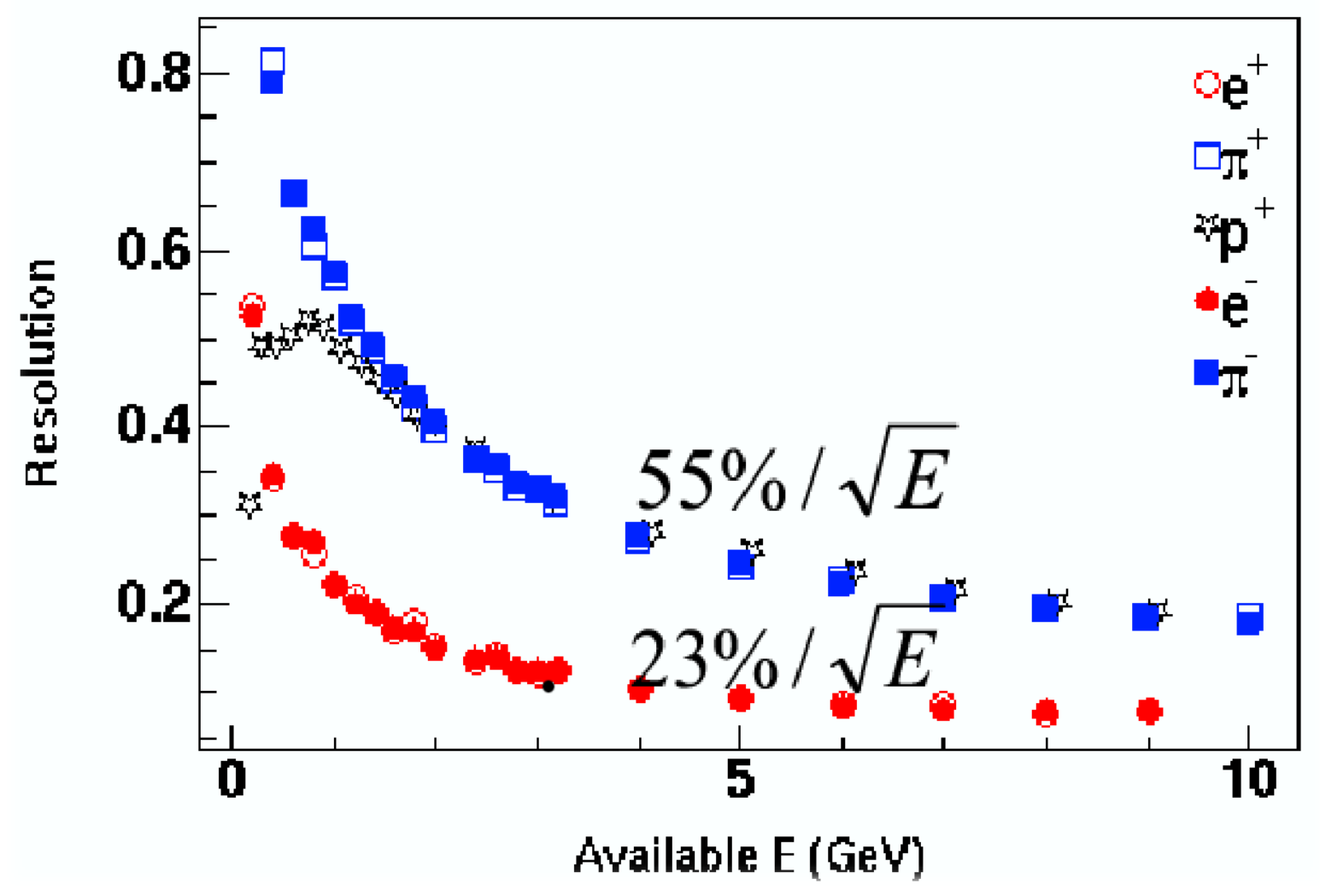

Figure 4.8: Hadronic and electromagnetic shower energy resolution as a function of available energy[43]. 


\subsection{MONTE CARLO EVENT SIMULATION}

A perfect detector would give the true value of the measured quantities, but due to detector resolution the measured quantities are smeared. Monte Carlo(MC) simulation attempts to correct for effects of resolution, selection cuts and detector geometry. In this analysis, the MC simulation has also been used to apply a correction to the flux that has been described later in section 7.2. The MINOS MC event simulator has three main components: production of neutrinos, interaction of neutrinos in and around the detector and propagation of the particles through the detector.

NuMI beamline is modeled using FLUKA[33] that simulates the production of secondary hadrons and the subsequent particle decay. The particles are propagated using GEANT3[7] simulation of the beamline. The largest uncertainty in the beamline simulation is due to the uncertainty in the yield of secondary hadrons as a function of the longitudinal and transverse momentum of the parent hadron. The size of this uncertainty, which is not known well known due to the lack of hadron production data, is about $8 \%$ in the beam peak and increases to $15 \%$ at higher energy. The large uncertainty on the simulated flux justifies the need to extract the flux directly from data.

Neutrino interactions are simulated using NEUGEN-v3 [37] which has two important components: simulation of the neutrino interaction and re-interaction of the hadronic particles with the neighboring nucleons. The cross section calculations are constrained by charged current cross section measurement from other experiments. Three separate models are used to simulate the QEL, RES and DIS constituents that calculate the form factors for QEL(Eq. 1.15) and RES interactions(Eq. 1.18) and structure functions(Eq. 1.30) for DIS interactions. There is significant overlap of the RES and DIS cross section below $W$ of $1.7 \mathrm{GeV}$. This transition region is handled by expressing the cross section as a sum of the pure resonance cross section and a non-resonance contribution from the DIS model that has been tuned to describe the neutrino data in this region. The interaction of final state hadronic particles is simulated in NEUGEN using data from pion 
and proton scattering experiments. The particles produced in the neutrino interaction are propagated through the detector using GEANT3 [7]. The following sections describe each component of NEUGEN.

\subsection{QUASI ELASTIC}

The simulation of QEL interactions, which dominate the low energy region, is based on the Llewellyn-Smith[56] model. The general form of quasi-elastic differential cross section has been shown earlier in Eq. 1.16. In order to calculate the cross section, we need to know the form factors $F$. The scalar $\left(F_{S}\right)$ and tensor $\left(F_{T}\right)$ form factors can be set to zero because they are second class currents and violate G-parity. Using Conserved Vector Current hypothesis, the vector part of the weak current can be related to the electromagnetic form factors $\left(G_{E}^{V}\left(q^{2}\right)\right.$ and $\left.G_{M}^{V}\left(q^{2}\right)\right)$,

$$
\begin{aligned}
& F_{V}^{1}\left(q^{2}\right)=\left(1-\frac{q^{2}}{4 M^{2}}\right)^{-1}\left[G_{E}^{V}\left(q^{2}\right)-\frac{q^{2}}{4 M^{2}} G_{M}^{V}\left(q^{2}\right)\right], \\
& \xi F_{V}^{2}\left(q^{2}\right)=\left(1-\frac{q^{2}}{4 M^{2}}\right)^{-1}\left[G_{M}^{V}\left(q^{2}\right)-G_{E}^{V}\left(q^{2}\right)\right] .
\end{aligned}
$$

$G_{E}^{V}\left(q^{2}\right)$ and $G_{M}^{V}\left(q^{2}\right)$ have the form

$$
G_{E}^{V}\left(q^{2}\right)=\frac{1}{\left(1-\frac{q^{2}}{M_{V}^{2}}\right)^{2}}, \quad G_{M}^{V}\left(q^{2}\right)=\frac{1+\mu_{p}-\mu_{n}}{\left(1-\frac{q^{2}}{M_{V}^{2}}\right)^{2}}
$$

where, $M_{V}$ is the vector mass, $\mu_{p}$ is the proton magnetic moment and $\mu_{n}$ is the neutron magnetic moment. These parameters are determined from electron scattering experiments.

The $q^{2}$ dependence of the axial formfactor $F_{A}$ is not known and is assumed to have a dipole parametrization

$$
F_{A}\left(q^{2}\right)=\frac{F_{A}(0)}{\left(1-\frac{q^{2}}{M_{A, Q E L}^{2}}\right)^{2}} .
$$

$F_{A}(0)$ has been measured from neutron beta decay experiments with high precision. $M_{A}$ value is determined from fits to bubble chamber experiments[22, 32, 29] and K2K[40] results. Table 5.2 


\begin{tabular}{|c|c|c|}
\hline Parameter & Value & Error \\
\hline \hline$M_{V}$ & 0.84 & small \\
\hline$\mu_{p}$ & 2.793 & small \\
\hline$\mu_{n}$ & -1.913 & small \\
\hline$F_{A}\left(q^{2}=0\right)$ & 1.267 & 0.004 \\
\hline$M_{A}$ & 0.99 & 0.15 \\
\hline
\end{tabular}

Table 5.1: Parameters for the QEL model used in NEUGEN. The right hand column shows the errors on these parameters. The parameters have been taken from Ref.[8].

shows the QEL parameter values and the associated uncertainties. The error on the $M_{A}$ parameter is large $(15 \%)$ and arises primarily from two factors. A nuclear correction is applied to the bubble chamber data using Fermi-gas model because it was collected on deuterium and MINOS uses iron. The nuclear correction itself has an error of the order of $10 \%$ at the cross section level. In addition, the $M_{A}$ value was obtained from fits using $Q^{2}$ shape information alone without using any flux information, which also contributed to the uncertainty. Fig. 5.2 shows the QEL cross setion (red line) for neutrino and antineutrino interactions and the effect of shifting $M_{A}$.

\subsection{RESONANCE}

The intermediate energy resonance(RES) interactions are simulated according to the Rein-Sehgal model [54] that is based on the baryon resonance model by Feynman, Kislinger and Ravndal[36]. This involves finding an expression for the $<N\left|F_{ \pm, 0}\right| N^{*}>$ term in Eq. 1.21. According to the FKR

model, a nucleon is treated as a bound state of three quarks in a harmonic oscillator potential and the excitation of these states are the observed resonances. A harmonic oscillator hamiltonian is

$$
H=\frac{1}{2 m} P^{2}+\frac{1}{2} m \omega_{0}^{2} X^{2} .
$$




\begin{tabular}{|c|c|c|}
\hline Parameter & Value & Error \\
\hline \hline$M_{A}$ & 1.12 & 0.17 \\
\hline$\Omega$ & 1.05 & small \\
\hline
\end{tabular}

Table 5.2: Parameters for the resonance model used in NEUGEN. The right hand column shows the errors on these parameters. The parameters have been taken from [45].

This can be rearranged and written as

$$
2 m H=P^{2}+\Omega^{2} X^{2}
$$

where, $\Omega^{2}=m^{2} \omega_{0}^{2}$ removes the quark mass dependence. Using Eq. 5.5 as a starting point, the operator $K$ for three quark baryon model can be written as

$$
K=3\left(p_{a}^{2}+p_{b}^{2}+p_{c}^{2}\right)+\frac{1}{36} \Omega^{2}\left[\left(u_{a}-u_{b}\right)^{2}+\left(u_{b}-u_{c}\right)^{2}+\left(u_{c}-u_{a}\right)^{2}\right]+\text { const } .
$$

Here $p_{a}^{2}\left(=p_{a \mu} p_{a}^{\mu}\right)$ is the square of the four-momentum of quark $a$ and $u_{a}$ is the conjugate position which means $p_{a \mu}=i \partial / \partial u_{a \mu}$.

Like QEL interactions, the axial component of the hadronic current is assumed to have a dipole parametrization

$$
F_{A}=\frac{1}{\left(1-\frac{q^{2}}{M_{A, R E S}^{2}}\right)^{2}} .
$$

The largest uncertainty is from the knowledge of $M_{A, R E S}$ value which was determined from fits to bubble chamber data. Table 5.2 shows the value of the model parameters and the associated errors.

Resonance contribution from 16 channels are included in the Rein-Sehgal model. Resonance cross section is calculated from the Rein-Sehgal model below a $W$ of $1.7 \mathrm{GeV}$, above this $W$ there is a contribution from DIS cross section as well that is explained in section 5.4. Fig. 5.2 shows the RES cross setion (green line) for neutrino and antineutrino interactions and the effect of shifting $M_{A, R E S}$. 


\subsection{DEEP INELASTIC SCATTERING}

DIS interactions, described in section 1.2.2.3, are simulated by the effective leading order model by Bodek and Yang[5]. To account for contributions from higher orders an improved scaling variable $\xi_{W}$ is used. The expression for $\xi_{W}$ can be derived from the effective scaling variable shown in Eq. 1.40

$$
\xi_{W}=\frac{2 x\left(Q^{2}+M_{f}^{2}+B\right)}{Q^{2}\left[1+\sqrt{1+4 M^{2} x^{2} / Q^{2}}\right]+2 A x},
$$

where, $M_{f}$ is the mass of the quark in the final state $\left(M_{f}=0\right.$ except for charm production). The parameter $A$ accounts for the higher twist effects as well as target mass. The parameter $B$ accounts for the initial state quark transverse momentum and prevents $\xi_{W}$ from diverging at $Q^{2}=0$.

The GRV 98 LO [39] PDFs(Parton Distribution Function) are used as an input to this model. They are multiplied by $K$ factors in order to describe the low $Q^{2}$ data well

$$
\begin{aligned}
& K_{\text {Sea }}\left(Q^{2}\right)=\frac{Q^{2}}{Q^{2}+C_{S}} \\
& K_{\text {Valence }}\left(Q^{2}\right)=\left[1-G_{D}^{2}\left(Q^{2}\right)\right] \times \frac{Q^{2}+C_{v 2}}{Q^{2}+C_{v 1}}
\end{aligned}
$$

where, $G_{D}=1 /\left(1+Q^{2} / 0.71\right)^{2}$ is the proton elastic form factor. There are separate correction factors for up and down quarks. The Bodek-Yang GRV-98 PDFs freeze at a $Q^{2}$ value of $0.8 \mathrm{GeV}^{2}$, below this value the structure function is given as

$$
F_{2}\left(x, Q^{2}<0.8\right)=K\left(Q^{2}\right) \times F_{2}\left(\xi, Q^{2}=0.8\right)
$$

The physical boundaries of the model is determined by $Q^{2}>M_{p}^{2} G e V^{2}, W>M_{p} G e V$, where $M_{p}$ is the proton mass. 1

To determine the model parameters a fit is performed to the DIS data from charged lepton scattering experiments. The parameter values of the Bodek-Yang model are shown in Table 5.3. The error on these parameters are not readily available, so a study (described later in section 7.5.2 ) is performed that shifts each of these parameters and calculates the $\chi^{2}$ for structure function

\footnotetext{
${ }^{1}$ There are some additional corrections required by this model that have been discussed in Appendix B.
} 


\begin{tabular}{|c|c|c|}
\hline Parameter & Value & Error \\
\hline$A$ & 0.538 & 0.134 \\
\hline$B$ & 0.305 & 0.076 \\
\hline$C_{v 1 u}$ & 0.291 & 0.087 \\
\hline$C_{v 2 u}$ & 0.189 & 0.076 \\
\hline$C_{v 1 d}$ & 0.202 & small \\
\hline$C_{v 2 d}$ & 0.255 & small \\
\hline$C_{s u}$ & 0.363 & small \\
\hline$C_{s d}$ & 0.621 & small \\
\hline
\end{tabular}

Table 5.3: Parameters for the DIS model used in NEUGEN. The right hand column shows the errors on these parameters. The parameters have been taken from Ref.[5].

$F_{2}$. Fig. 5.1 shows the effect of shifting the Bodek-Yang parameters on $F_{2}$. Fig. 5.2 shows the DIS cross setion (blue line) for neutrino and antineutrino interactions and the effect of shifting the parameter $A$.

\subsection{RES-DIS OVERLAP REGION}

In NEUGEN, the resonance and DIS region is separated artificially below a $W$ value of $1.7 \mathrm{GeV}$. In order to handle this region properly, the resonance model accepts non-resonance contributions up to a "safe"(defined by $W>1.7 \mathrm{GeV}$ ) DIS regime. The total inelastic contribution can be written as

$$
\frac{d \sigma}{d \theta d E}=\frac{d \sigma^{R E S}}{d \theta d E}+\frac{d \sigma^{D I S}}{d \theta d E}
$$

The resonance part is given by

$$
\frac{d \sigma^{R E S}}{d \theta d E}=\sum_{i=1}^{n r e s} \frac{d \sigma^{R E S}}{d \theta d E_{i}} \Theta\left(W_{c u t}-W\right) .
$$




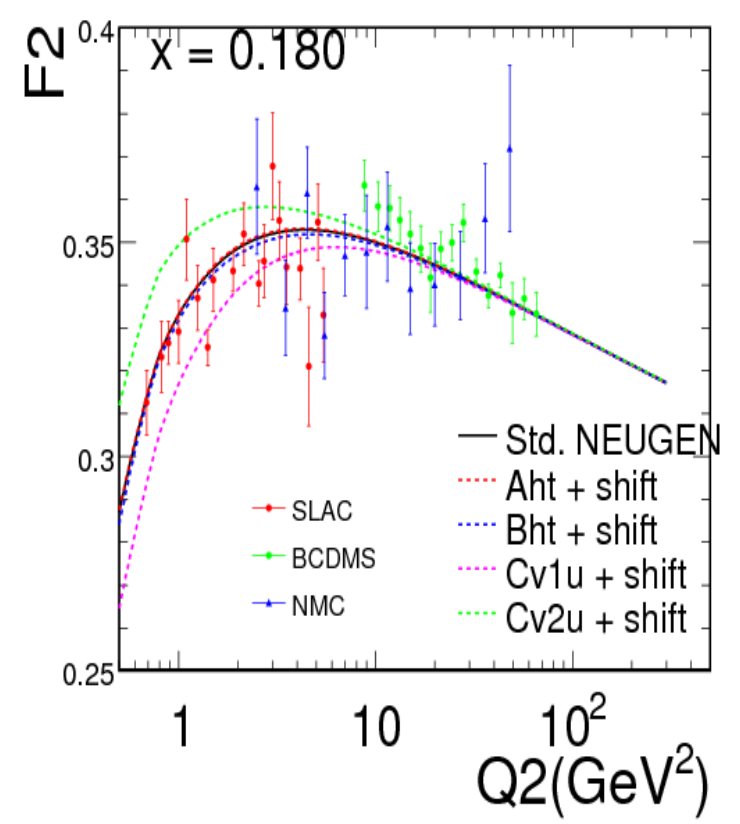

(a)

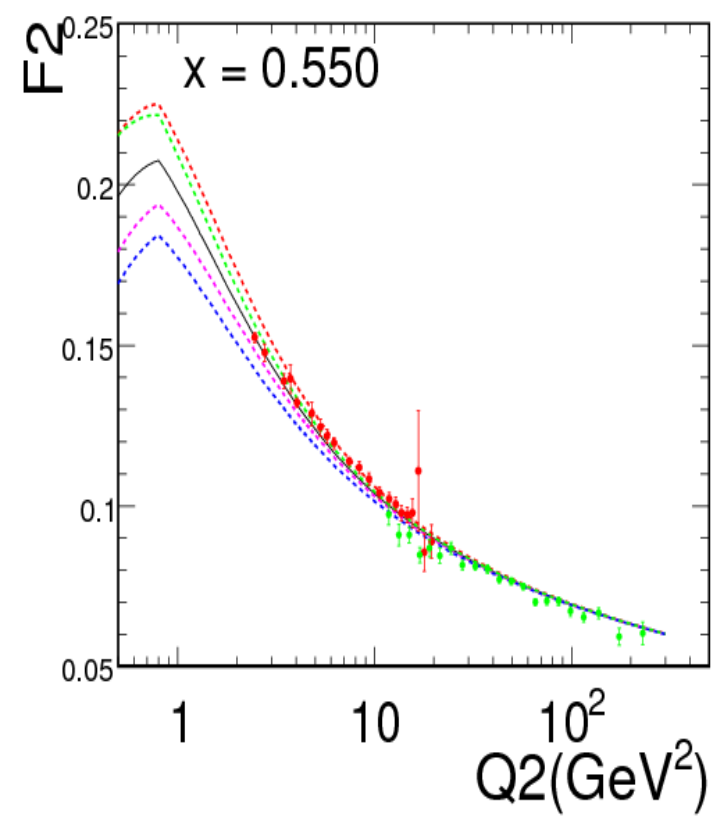

(c)

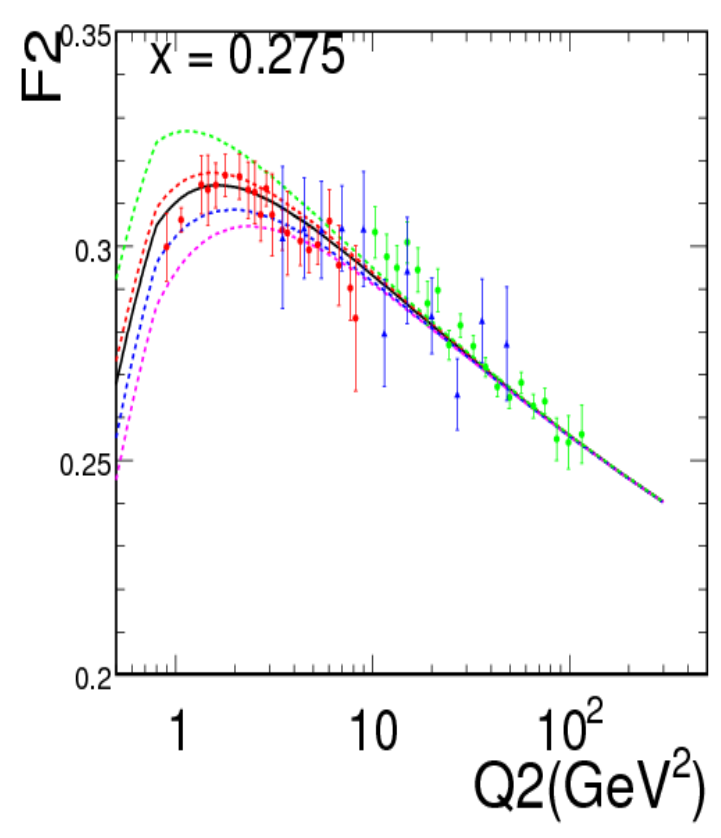

(b)

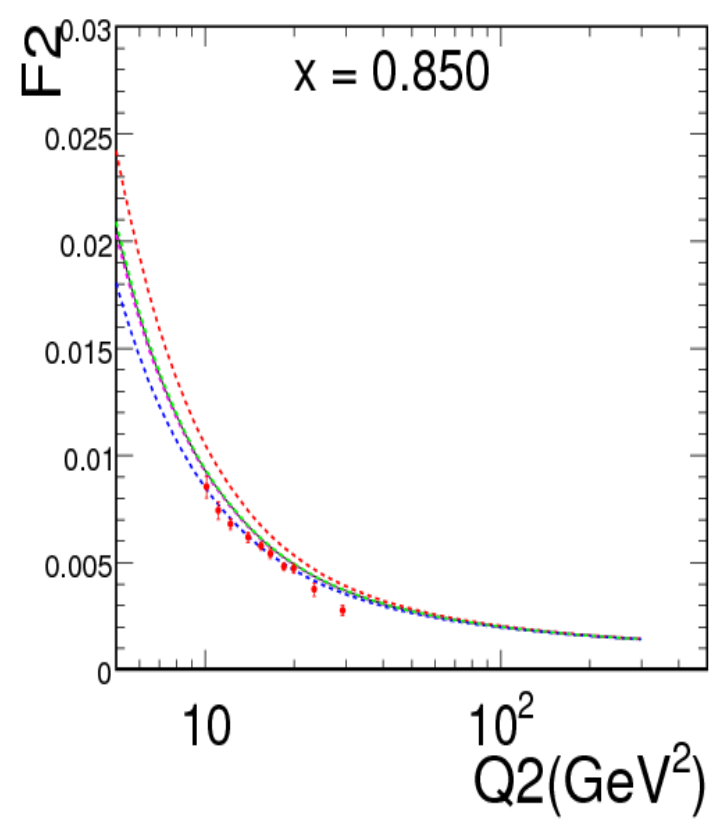

(d)

Figure 5.1: Effect of shifting the DIS parameter $A$ by $25 \%, B$ by $25 \%, C_{v 1 u}$ by $30 \%$ and $C_{v 2 u}$ by $40 \%$ on the charged lepton nucleon structure function $F_{2}$. The data points are from charged lepton scattering experiments. Each plot shows the $F_{2}$ versus $Q^{2}$ distribution for a given $x$ value. 
The summation is over the resonances in the Rein-Sehgal model. The DIS part is given by

$$
\frac{d \sigma^{D I S}}{d \theta d E}=\frac{d \sigma^{D I S}}{d \theta d E} \Theta\left(W-W_{c u t}\right)+\frac{d \sigma^{D I S}}{d \theta d E} \Theta\left(W_{c u t}-W\right) \sum_{n} f_{n}
$$

where, $n$ is the hadronic multiplicity of the system and the factor $f_{n}$ gives the contribution of a particular multiplicity channel to the total cross section. The factors $f_{n}$ are written as $f_{n}=r_{n} P_{n}$ where, $r_{n}$ is a tunable parameter and $P_{n}$ is the probability that a certain multiplicity final state will be produced, which is calculated from the KNO model [42].

The KNO model calculates the average multiplicity by using the expression

$$
<n>=a+b \log W^{2}
$$

where the parameters $a$ and $b$ depend on the initial state and is calculated from Bubble Chamber experiments. According to KNO scaling model, the probability $P(n)$ is only a function of the ratio $<n>/ n$. This function is determined from a parametrized fit to the data.

We verify that the choice of Wcut $(1.7 \mathrm{GeV})$ gives predictions without large discontinuities and in reasonable agreement with inclusive electron scattering data. The $r_{k}$ parameters are tuned from neutrino data(Durham Neutrino Database [57]). There are $16 r_{k}$ parameters in all for the following combinations, two for $\mathrm{CC} / \mathrm{NC}$, two for the multiplicities $n=2$ and $n=3$ and four for the combinations of the initial states(proton/neutron and neutrino/antineutrino).

Fig. 5.2 shows the total simulated cross section(black line) for neutrino and antineutrino interactions. The Neugen predicted total cross section at high energy has an offset from the world cross section $\left(0.677 \pm 0.014 \mathrm{~cm}^{2} / \mathrm{GeV}\right)$ between $30-200 \mathrm{GeV}$. To correct for this, both the neutrino and the antineutrino cross section are multiplied with a factor of 1.032 . At energies above $20 \mathrm{GeV}$, the cross section is completely dominated by DIS interactions. At lower energies, there is contribution from QEL and RES interactions. At $3 \mathrm{GeV}$ for the neutrino cross section, QEL contributes 30\%, RES and DIS contributes 70\%. Fig. 5.3 shows the ratio of antineutrino to neutrino cross section as a function of energy. At high energies, the QEL and RES antineutrino and neutrino cross sections are almost equal and the ratio approaches 1 . For DIS interaction the ratio is $\sim 0.5$, discussion of this feature is given later in Chapter 8 . 


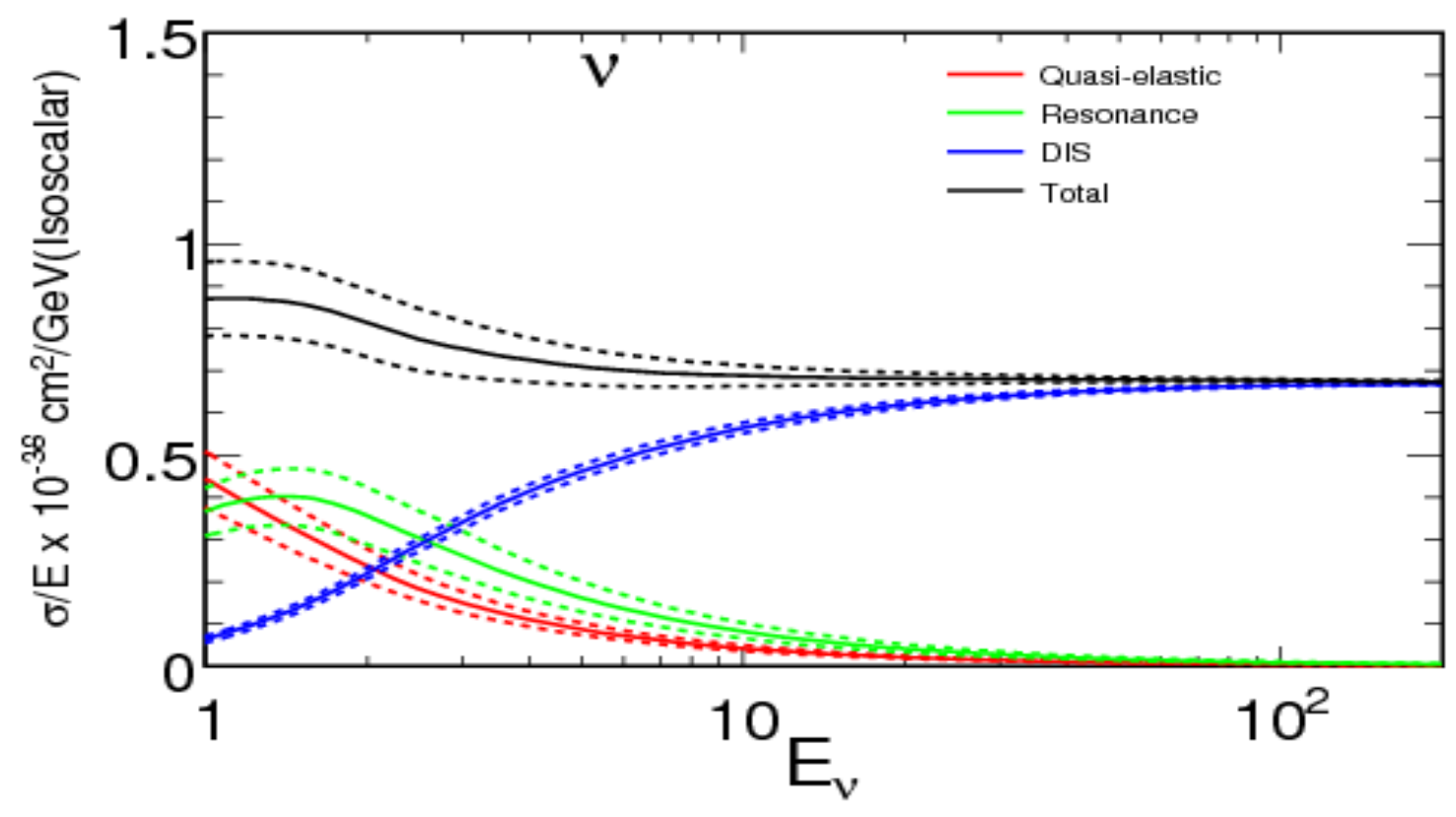

(a)

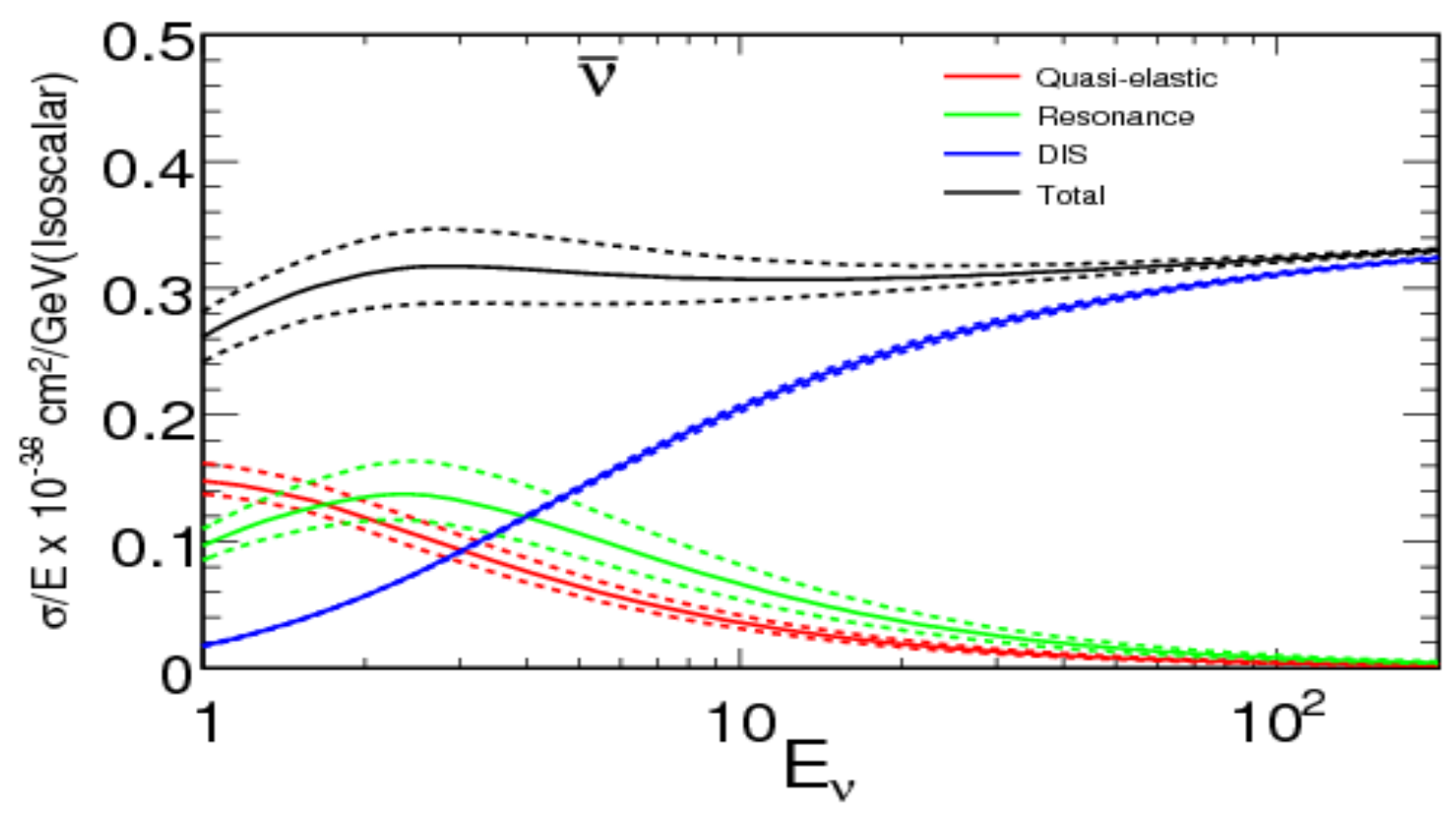

(b)

Figure 5.2: Neutrino(a) and antineutrino(b) cross section as a function of energy calculated from the model. The total cross section is shown in black, QEL in red, RES in green and DIS in blue. The dashed bands show the effect of modifying the model parameters. 


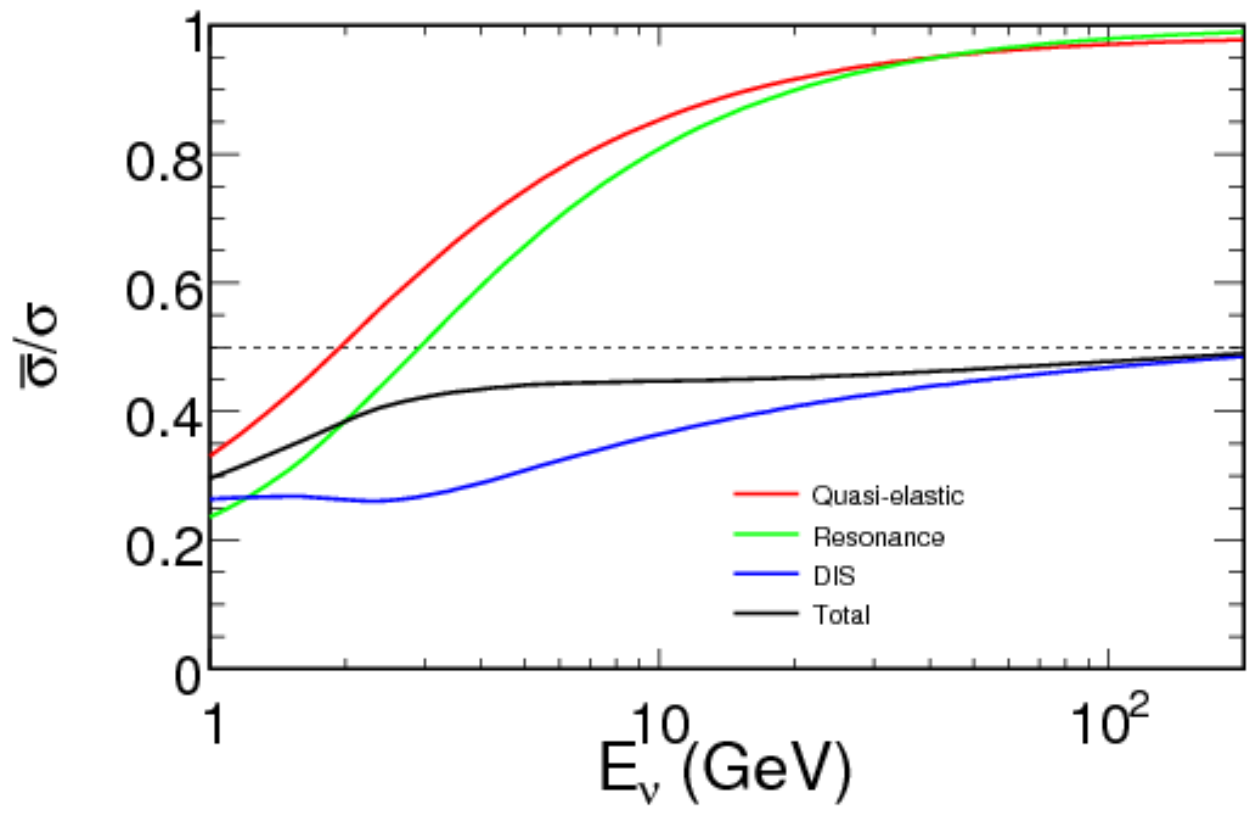

Figure 5.3: Ratio of antineutrino to neutrino cross section as a function of energy calculated from the model. The total cross section ratio is shown in black, QEL ratio in red, RES ratio in green and DIS ratio in blue. The dashed line at 0.5 shows the world average ratio between $30-200 \mathrm{GeV}$. 


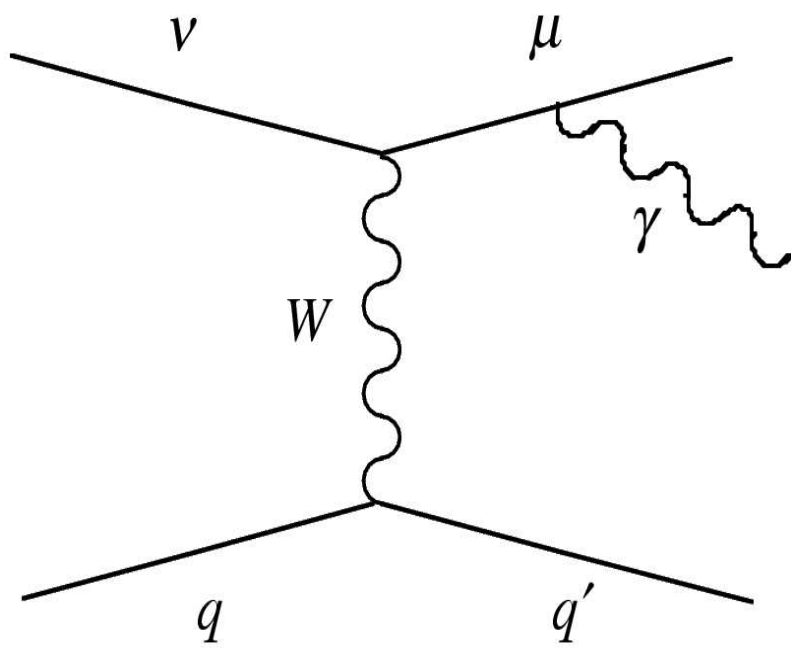

(a)

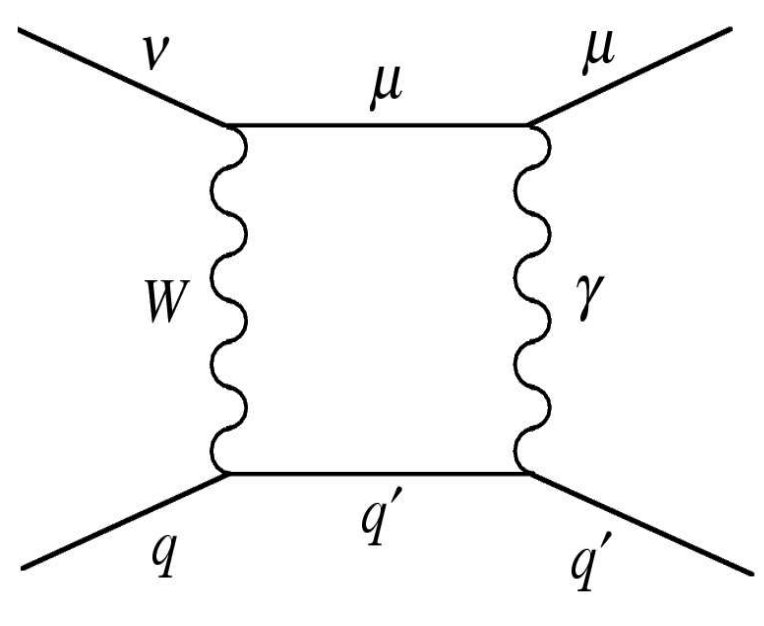

(b)

Figure 5.4: QED loop corrections applied to charged current neutrino interactions.

\subsection{RADIATIVE EFFECTS}

The radiative effects account for contributions to the cross section from emission of photons by charged particles. The default cross section model doesn't include radiative effects and they have been added as a correction based on calculations in reference[4]. Fig. 5.4 shows two of the loops used in this calculation.

Fig. 5.5 shows the radiative correction for differential cross section at an energy of $6 \mathrm{GeV}$. The correction is about $5 \%$ for low and high value of $x$, and is negligible at intermediate $x$ values. Fig. 5.6 shows the ratio of the radiative effect corrected cross section to the Born level cross section.

\subsection{INTRA NUCLEAR RESCATTERING}

The hadrons produced in a neutrino interaction can undergo further interactions with the neighboring nucleons before emerging from the nucleus, as shown in Fig. 5.7. Such processes can 


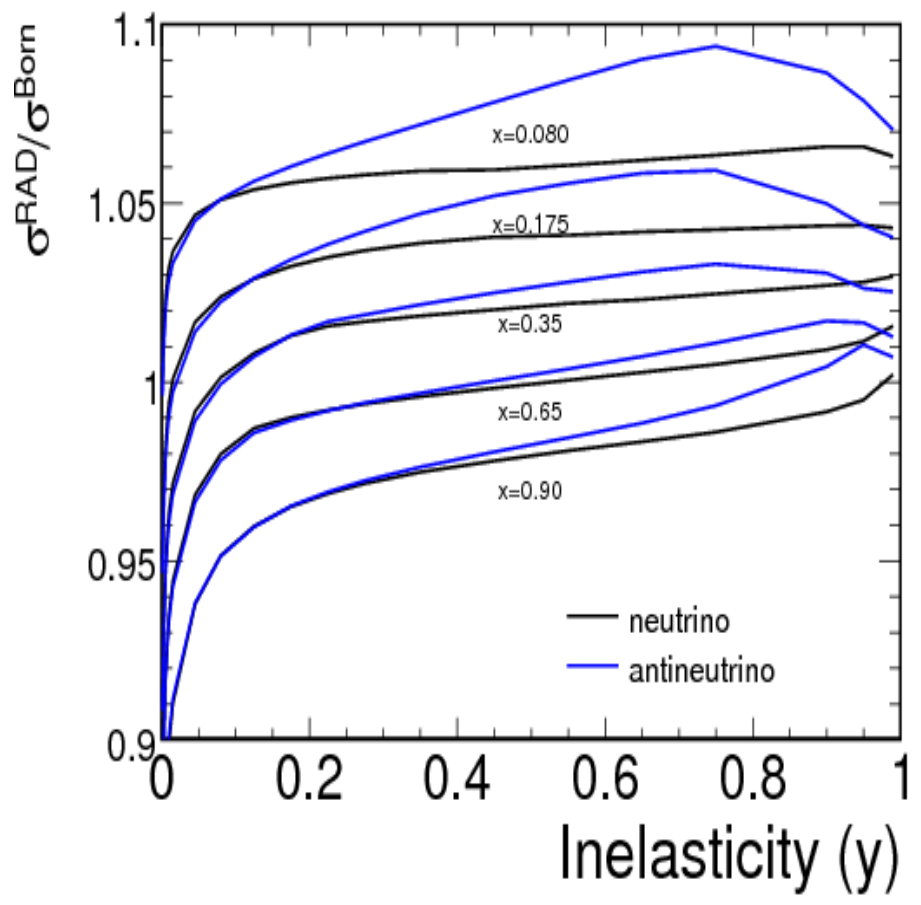

Figure 5.5: Radiative corrections for charged current neutrino(black) and antineutrino(blue) differential cross section for incident energy of $6 \mathrm{GeV}$ as a function of $y$ for various $x$ values. 


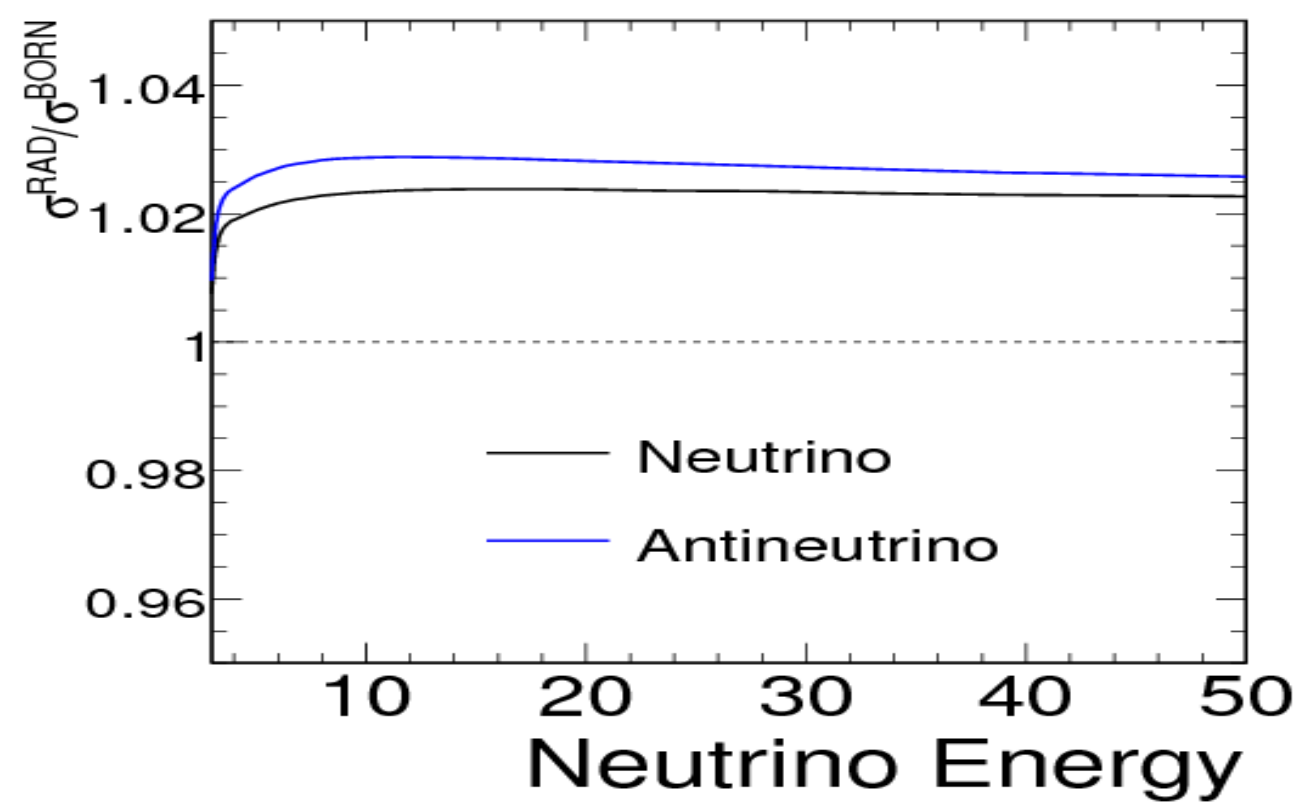

Figure 5.6: Ratio of radiative effect corrected total cross section to the Born level total cross section as a function of neutrino energy. 
significantly modify the visible energy of the hadronic shower and hence should be modeled well.

In NEUGEN model, each hadron is treated independently and allowed to propagate for a certain length known as the "formation length" without interacting. The formation length depends on the mass and the momentum of the hadron. The formation time $0.342 \mathrm{fm} / \mathrm{c}$ is used as an input parameter to the model [2]. The probability of a collision occurring before exiting the nucleus is calculated from the hadron mean free path in nuclear matter which is determined from external data. If no collision occurs, the hadron exits from the nucleus. If a collision occurs, there are several possible interactions a hadron can undergo. One possibility is that it undergoes an elastic/inelastic interaction in which no new particle is produced but only the energy changes. The other possibilities are that the initial hadron interacts to produce new particles, for example, a charged pion can undergo a charge exchange producing a neutral pion, or it can interact to produce multiple charged pions, or it can get completely absorbed and produce nucleons. These new particles are not subjected to re-interaction. These effects are simulated by a cascade Monte Carlo using $\pi N$, $p N$ and $\pi F e$ and $p F e$ scattering data(Fig. 5.8) and benchmarked against $v D$ and $v N e$ scattering data[49]. The uncertainty is determined (discussed in section 7.5) from the constraints on the parameters from external data and from evaluating the uncertainty associated with key assumptions in the model. 


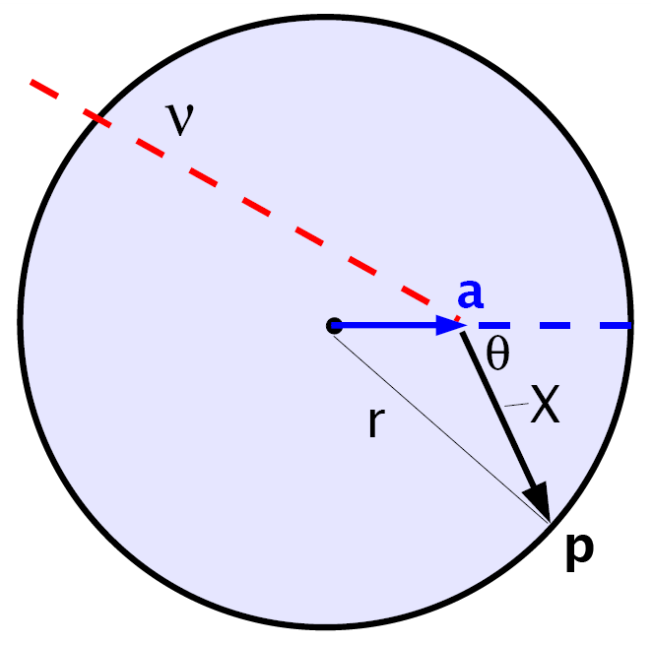

Figure 5.7: A neutrino induced hadronic shower particle is created at an angle $\theta$ with momentum p. The blue arrow joins the center of the nucleus to the interaction vertex a and the black arrow shows the shower particle direction. $\mathrm{X}$ is the distance traveled by the hadronic particle before it emerges from the nucleus [44]. 


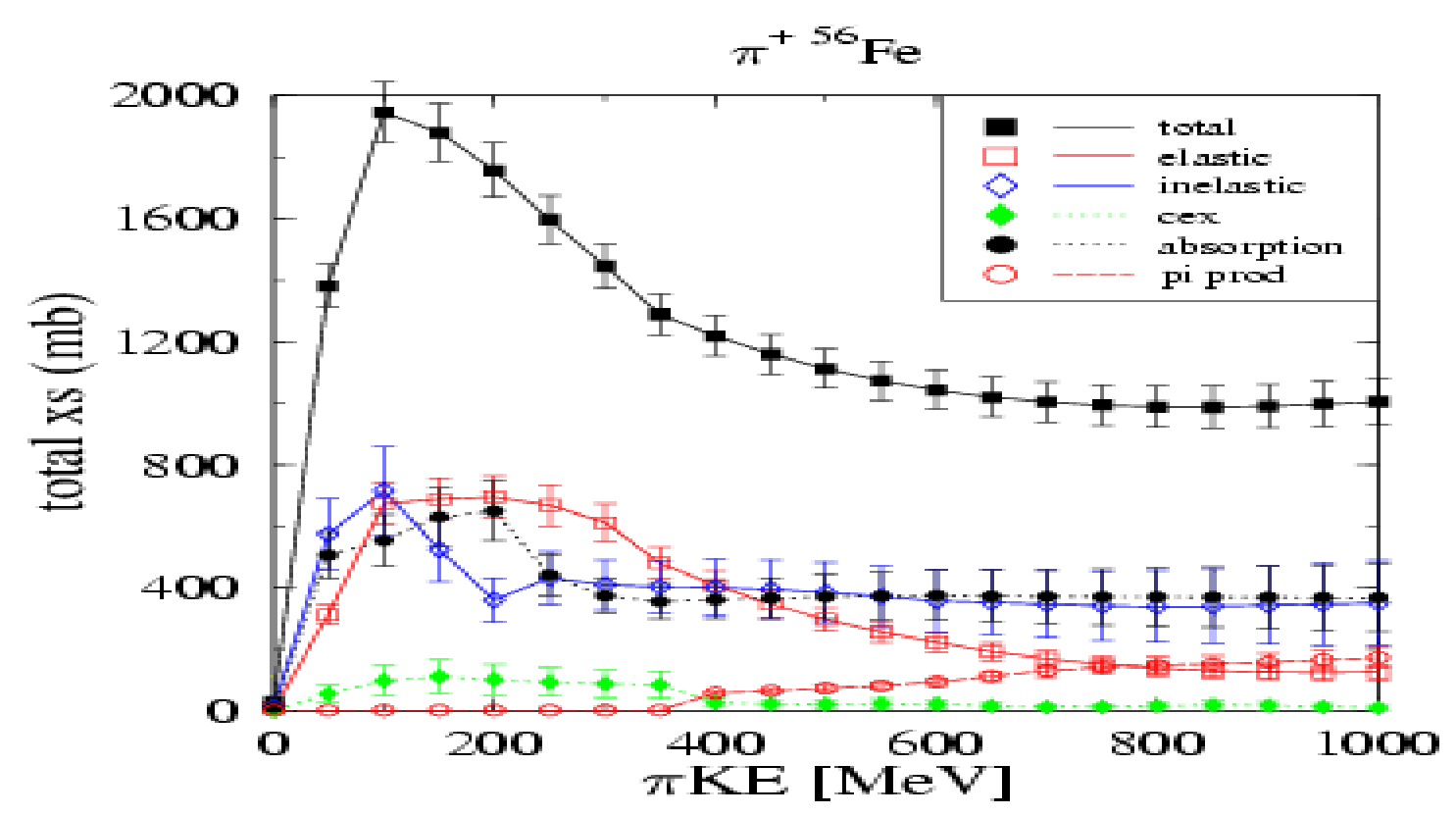

(a)

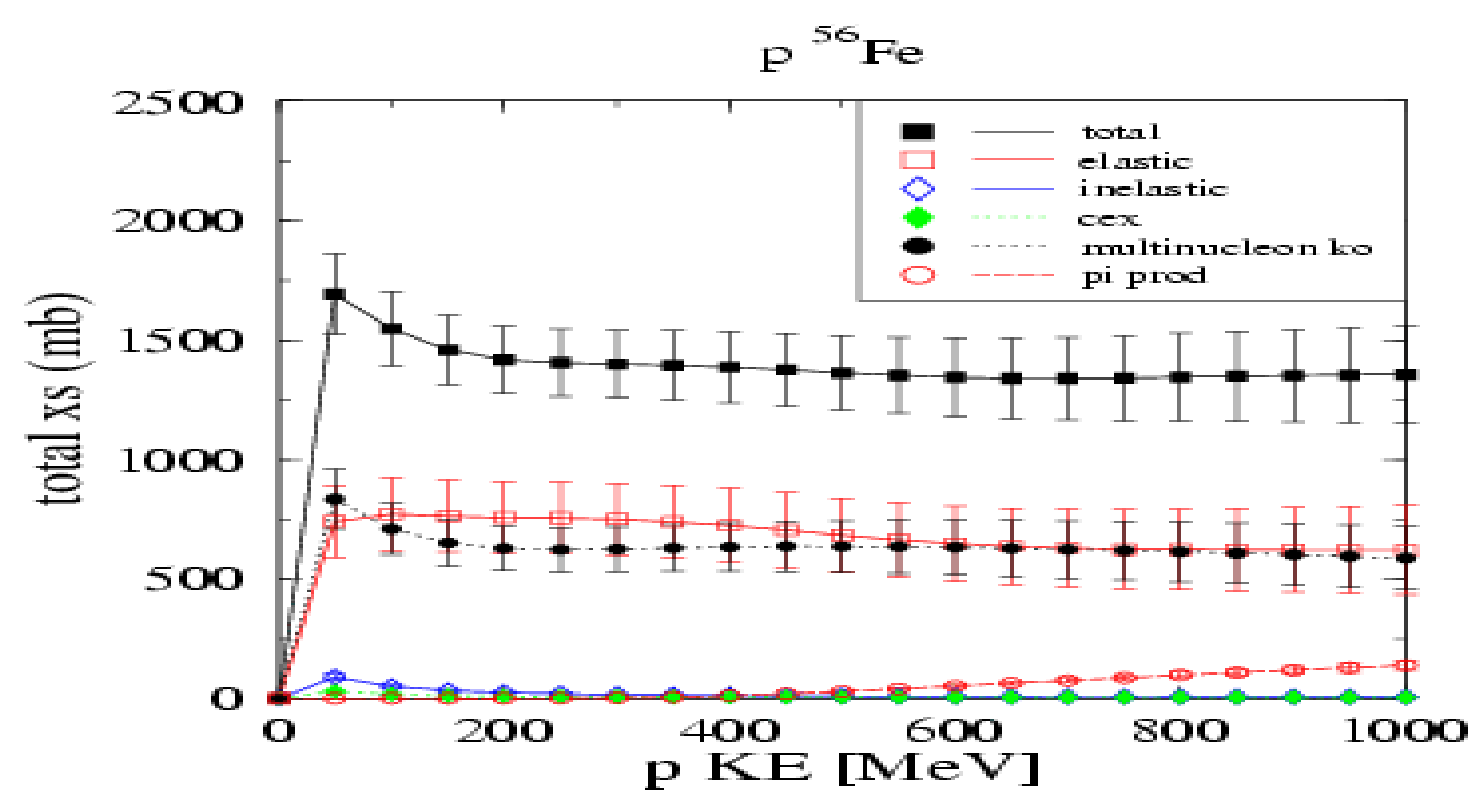

(b)

Figure 5.8: Interaction cross section for pion(a) and proton(b) as a function of kinetic energy from experimental data $[13,10]$. The total cross section is shown by the black line and the different contributions are shown in color. 


\subsection{DATA AND EVENT SELECTION}

The data sample used in this analysis is collected in the LE10/185 kA mode between June 2005

and April 2007 and corresponds to an exposure of $2.45 \times 10^{20}$ PoTs. Fig. 6.1 shows the number of protons on target as a function of time over this period. The MC sample is almost double in size, corresponding to a $4.4 \times 10^{20}$ PoTs exposure.

The charged current event selection criteria chooses a sample within the fiducial volume that has a signature muon track with well measured momentum and charge. Same selection criteria are applied to the data and MC event sample. This chapter describes the $\mathrm{CC}$ sample selection, background contamination and data and MC simulation comparison. A special set of data was collected with reversed magnetic field polarity in the detector. The last section of this chapter describes this dataset that evaluates the effect of modeling, reconstruction and smearing on the analysis.

\subsection{EVENT SELECTION}

Before applying any analysis specific selections, generic "beam quality" checks are made to ensure that each beam spill has at least $0.5 \times 10^{12}$ PoTs and the magnetic horn current is between $155 \mathrm{kA}$ and $200 \mathrm{kA}$. It is also required that the horizontal(vertical) width of the beam spot is between 0.1 $\mathrm{mm}$ and $2.2 \mathrm{~m}(0.1 \mathrm{~m}$ and $5 \mathrm{~mm})$ and the position of the beam is within $2 \mathrm{~mm}$ from the center of the target. These criteria are necessary because the MC doesn't simulate an increased(or reduced) beam size and off-target neutrino beams.

A CC sample is selected using the following criteria: 


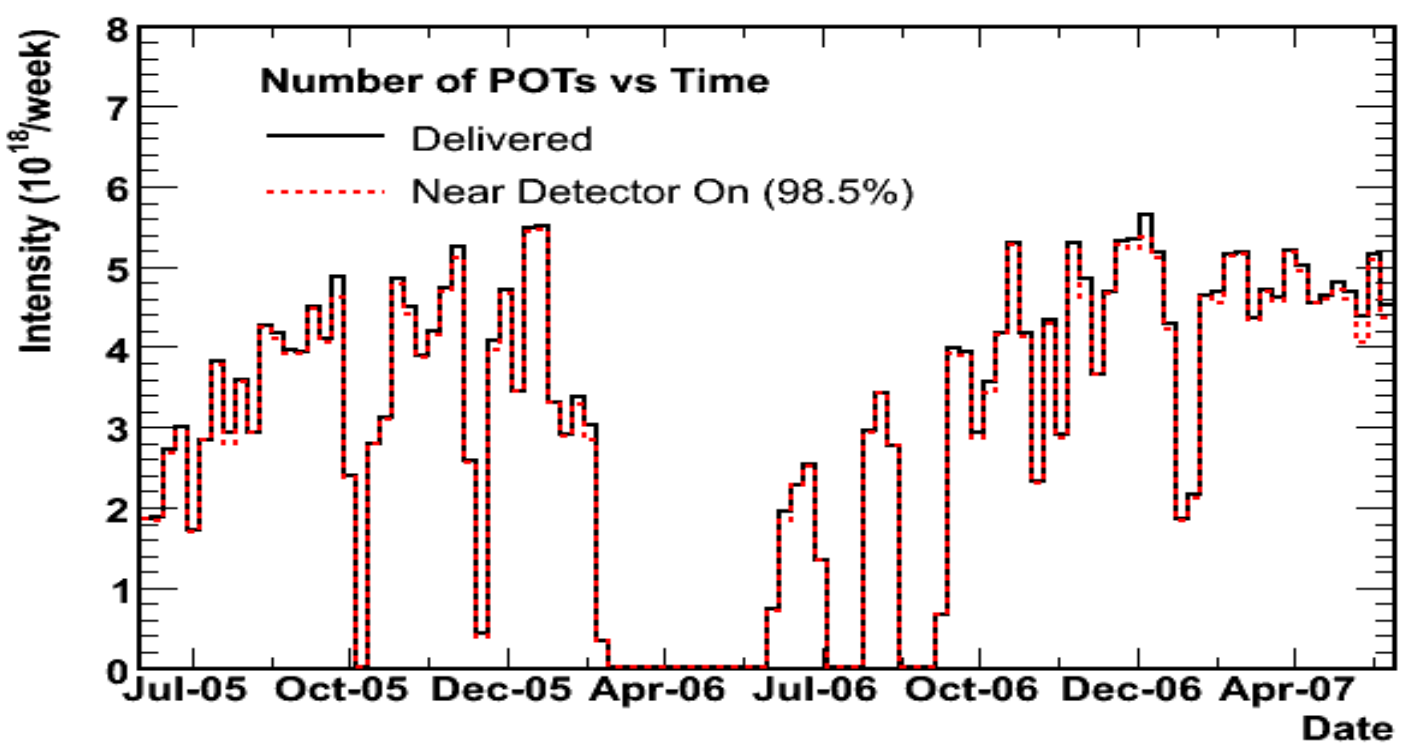

Figure 6.1: Number of PoTs as a function of time over a period of two years.

1. Fiducial volume - Selects events with vertices between $0.6 \mathrm{~m}-4.0 \mathrm{~m}$ in the calorimeter region and within $0.5 \mathrm{~m}$ from the edge of the instrumented region in the transverse view. It is also required that the radial distance of the event vertex be greater than $0.8 \mathrm{~m}$ from the magnetic coil hole. The fiducial length cut prevents showers from leaking into the spectrometer region which has coarser sampling. Fig. 6.2 shows the selected fiducial volume.

2. Track Energy - Requires a minimum energy of $1.5 \mathrm{GeV}$ for the longest track in the event. The track energy cut rejects Neutral Current(NC) background events populating the low energy bins and poorly reconstructed muons. Fig.6.3 shows the effect of applying a minimum muon energy criterion on the fractional NC contamination. The background reduces with increasing muon energy cut. The minimum muon momentum is chosen to be $1.5 \mathrm{GeV}$ because above below value the contamination increases significantly.

3. Coil hole - Requires that a minimum of $95 \%$ of hit strips in the track be more than $0.3 \mathrm{~m}$ from the coil hole at closest approach. This cut minimizes dependence on the accuracy of the coil 
hole model. Fig. 6.4 shows the resolution of muon tracks as a function of radial distance from the coil hole. Three categories of tracks are shown; those that have one strip, $2 \%$ of their strips and $5 \%$ of their strips within a certain radial distance. Resolution is better for tracks that spend a smaller fraction of their time near the coil hole. Above a radial distance of $0.3 \mathrm{~m}$ the resolution becomes constant and is similar for the three cases. Tracks that spend $95 \%$ of their time within this radial distance are selected.

4. Track Quality - Requires tracks to have a convergent fit and the resulting error on measured charge to momentum ratio $(q / p)$ to be less than $30 \%$. The error is determined from the covariance matrix of the Kalman Filter (see section 4.0.4). This criteria removes tracks that have a large error on $q / p$. Tracks with $q / p<0(q / p>0)$ are considered to be originating from $v_{\mu}$ $\mathrm{CC}\left(\bar{v}_{\mu} \mathrm{CC}\right)$ interactions. In addition, the difference in the track beginning plane in the two views must be no more than 5 planes. Fig. 6.5 shows the $\mathrm{z}$ vertex resolution as a function of the difference in the beginning plane in the $\mathrm{u}$ and $\mathrm{v}$ view. The resolution is better for tracks with a smaller difference between the beginning plane in the two views. We choose this value to be 5 planes.

5. Neutrino Energy - Removes events with neutrino energy less than $3 \mathrm{GeV}$ for the neutrino sample and less than $5 \mathrm{GeV}$ for the antineutrino sample. We use the MC event sample to apply an "acceptance correction" that corrects for the effects of event selection, smearing and detector geometry as described in section 6.4. The acceptance correction is large at lower energies as shown in Fig. 6.12, which requires a minimum energy cut to be applied. Events above $50 \mathrm{GeV}$, which are small in number, are not used because smearing effects are large and not well understood.

\subsection{EVENT SUBSAMPLES}

The event sample is divided into two categories based on whether the track stops in or exits the detector. Any track that stops at a distance of greater than $0.5 \mathrm{~m}$ from the edge of the instrumented region in the transverse view and within $15 \mathrm{~m}$ in the longitudinal view, is taken to be in the "stopping sample". Between $15 \mathrm{~m}$ and $16.6 \mathrm{~m}$ (end of the detector) there are 6 fully instrumented planes, 


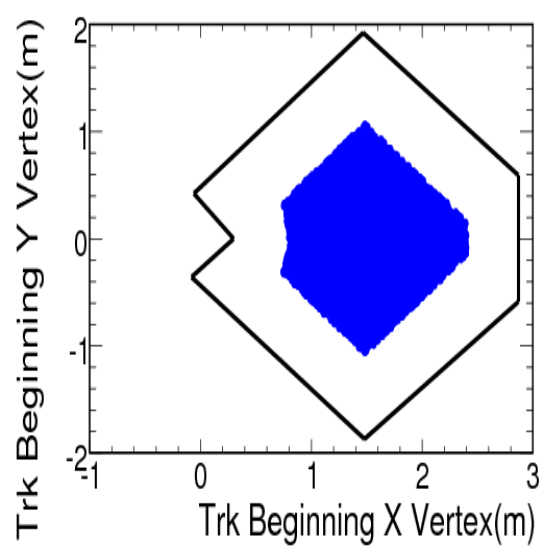

(a)

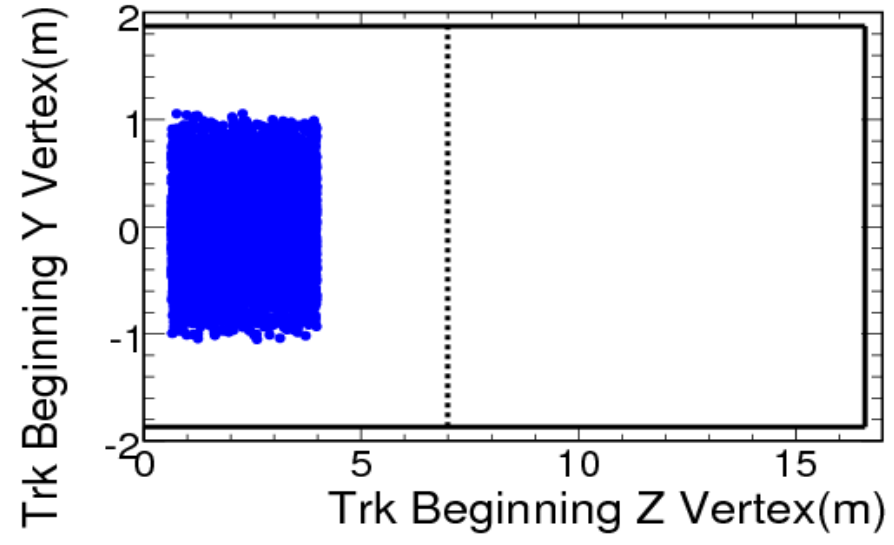

(b)

Figure 6.2: Fiducial volume in the transverse view(a) and the longitudinal view(b) is shown by the blue shaded region. The solid black line shows the outline of the instrumented region and the dashed line shows the end of the calorimeter region.

hence choosing the track $\mathrm{z}$ end value at $15 \mathrm{~m}$ ensures that there are no exiting tracks that have been accidentally identified as stopping tracks. The stopping and exiting tracks are further differentiated based on whether they end in the upstream or downstream region (see Fig. 2.5). This is necessary because sampling in the two regions are different.

Fig. 6.6 shows the muon energy distribution of neutrino and antineutrino subsamples. The upstream samples have lower energies than the downstream samples. In the standard magnetic field polarity, $\mu^{-}$s get focused and $\mu^{+}$s get defocused. As a result, the antineutrino sample has a larger fraction of exiting events compared to the neutrino sample. Fig. 6.7 shows the inelasticity distribution of neutrino and antineutrino subsamples. The upstream samples have higher inelasticity compared to the downstream samples because of their lower energy.

Table 6.1 and 6.2 show the size of the selected MC sample and the percentage contamination after applying the cuts listed above for neutrinos and antineutrinos respectively. The four samples in the tables have been arranged in increasing order of energy moving from left to right. The low energy samples for both neutrinos and the antineutrinos have higher NC contaminations because 


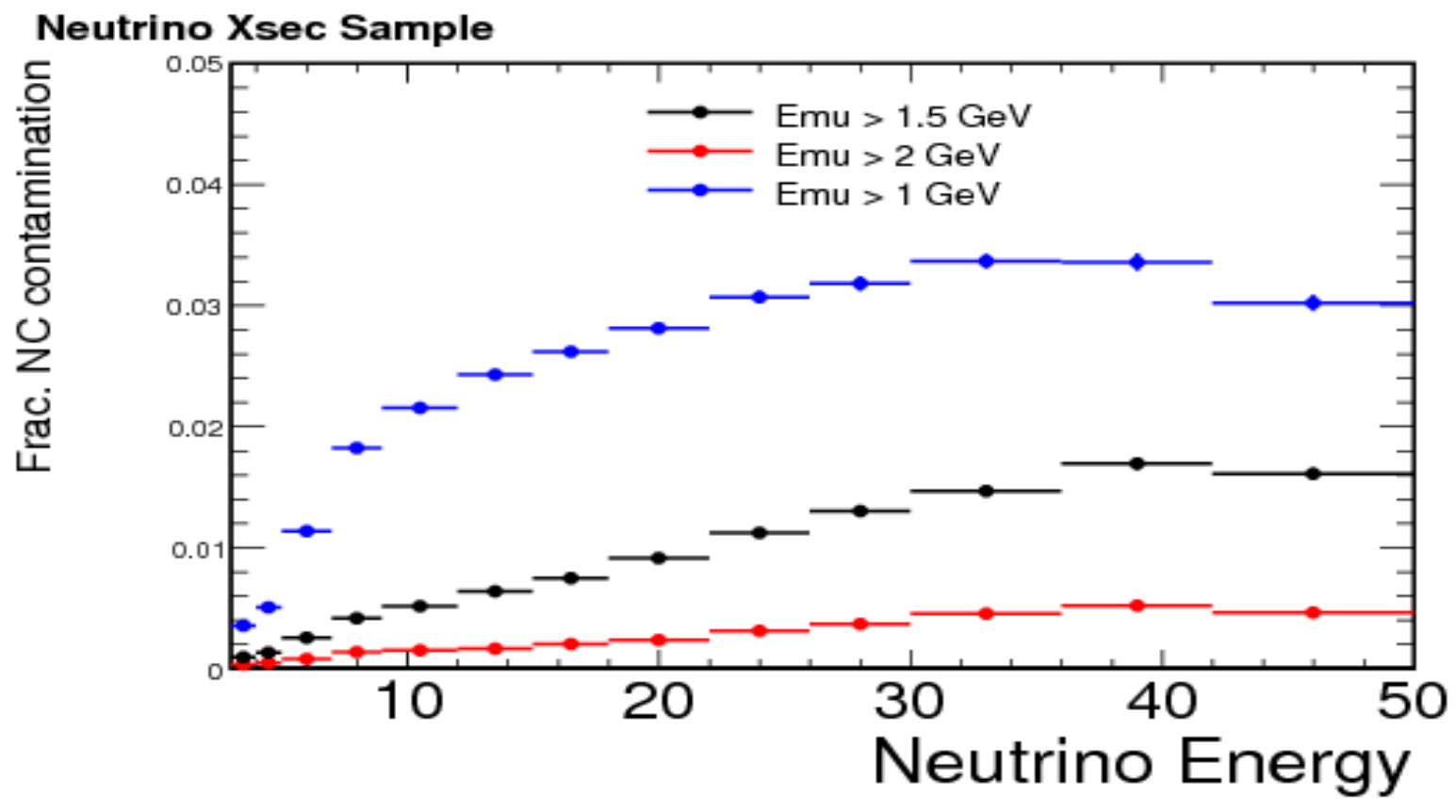

Figure 6.3: Effect of muon energy selection criterion on fractional NC contamination for neutrino $\mathrm{CC}$ sample as a function of energy. 


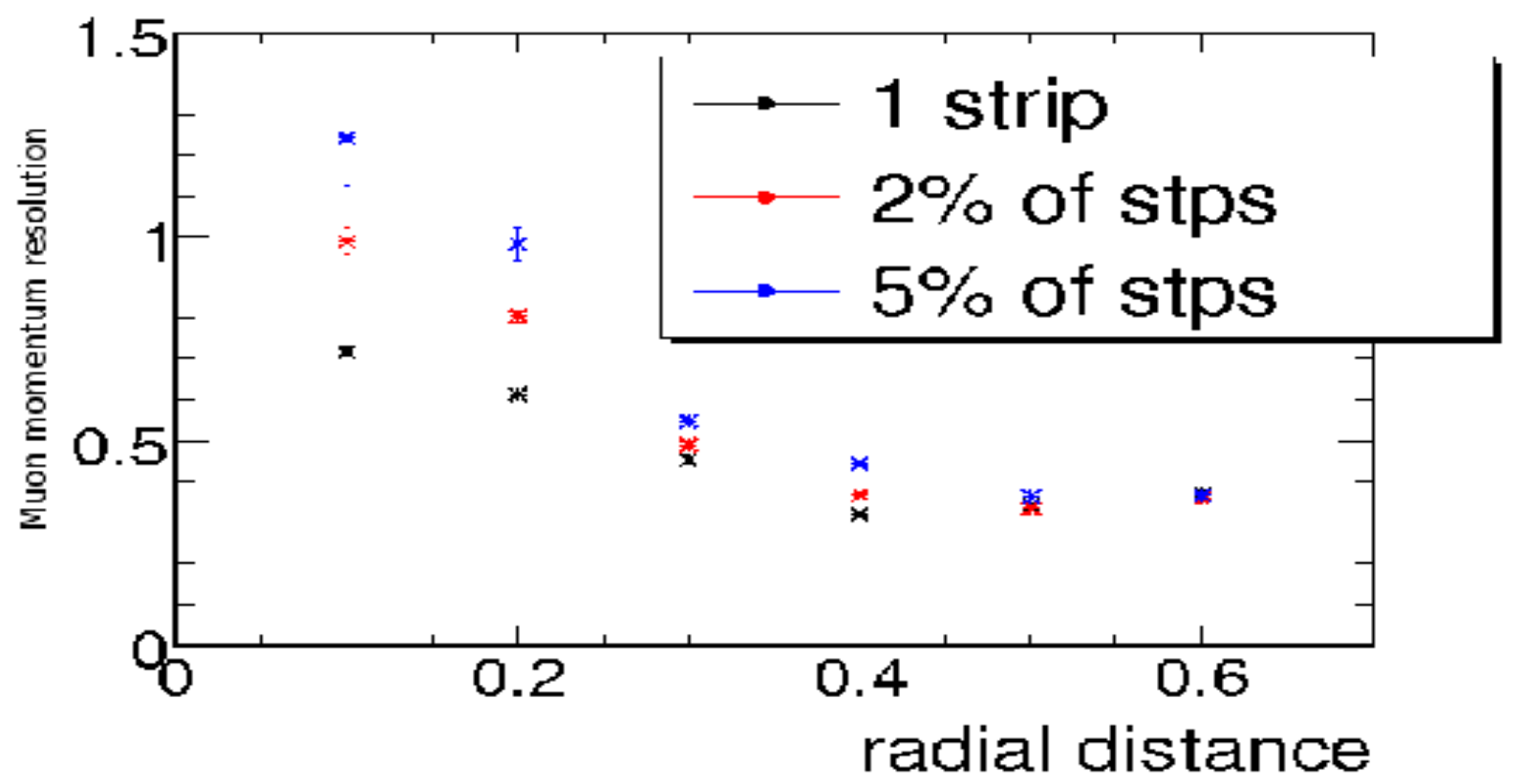

Figure 6.4: Muon momentum resolution as a function of radial distance from the coil hole. Three cases are shown, tracks that have only one strip(black line), tracks that have $2 \%$ of their strips(red line) and tracks that have $5 \%$ of their strips(blue) within the radial distance shown in the x-axis. 


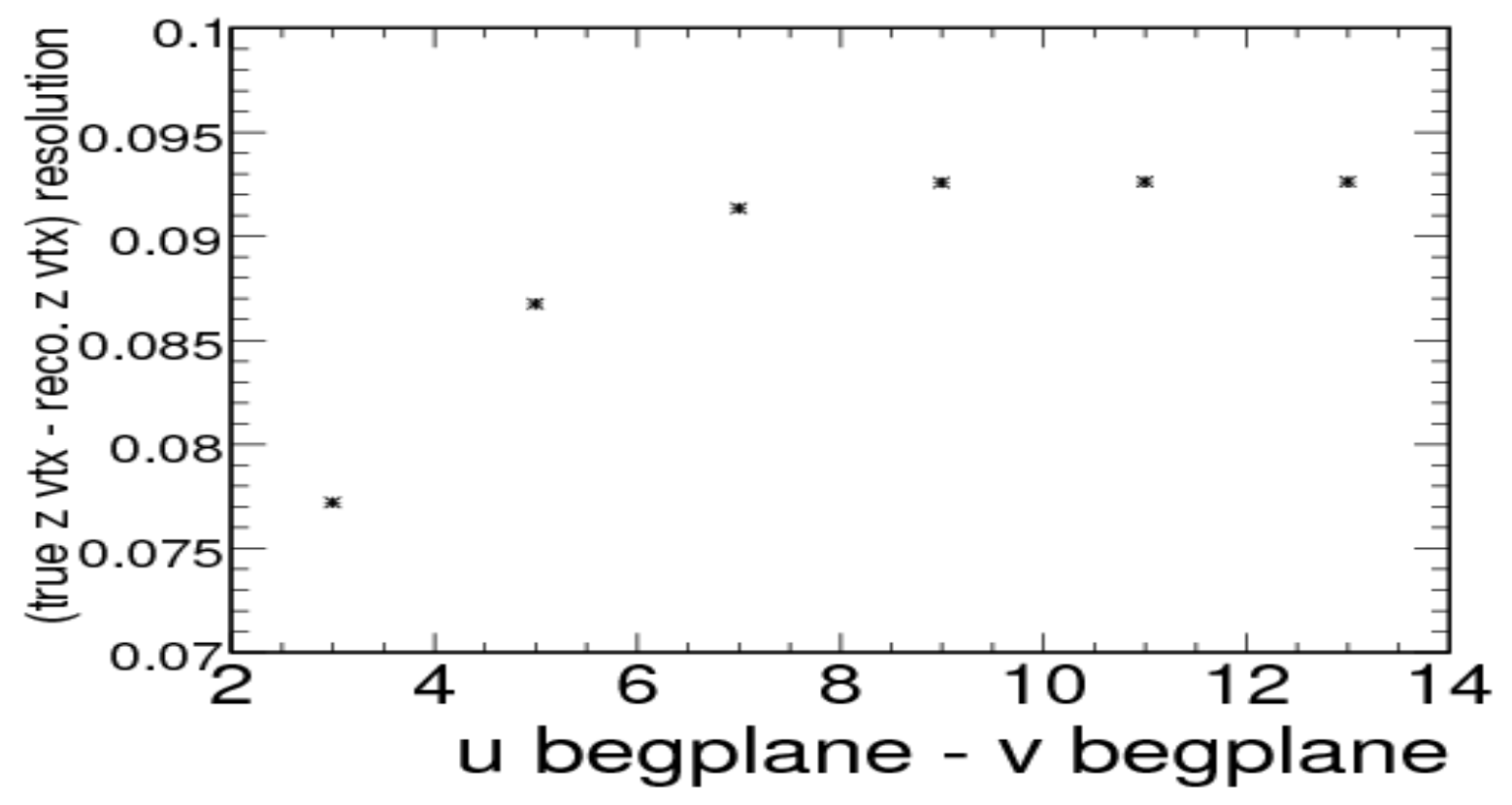

Figure 6.5: $\mathrm{Z}$ vertex resolution as a function of the difference between beginning plane in the $\mathrm{u}$ and $\mathrm{v}$ view(a). Distribution of the $\mathrm{z}$ vertex resolution(b) with the standard CC selection criteria. 


\begin{tabular}{|c|c|c|c|c|c|}
\hline $\begin{array}{c}\text { Track Charge }<0 \\
\text { (selected neutrinos) }\end{array}$ & $\begin{array}{c}\text { stopping } \\
\text { upstream }\end{array}$ & $\begin{array}{c}\text { exiting } \\
\text { upstream }\end{array}$ & $\begin{array}{c}\text { stopping } \\
\text { downstream }\end{array}$ & $\begin{array}{c}\text { exiting } \\
\text { downstream }\end{array}$ & Total \\
\hline \hline sample size & 280994 & 849991 & 235234 & $1.678 \times 10^{6}$ & $3.045 \times 10^{6}$ \\
\hline$\%$ contribution & 9.23 & 27.91 & 7.72 & 55.13 & 100 \\
\hline$\%$ NC & $3.21 \pm 0.03$ & $0.55 \pm 0.01$ & $0.15 \pm 0.01$ & $0.04 \pm 0.002$ & $0.48 \pm 0.004$ \\
contamination & & & & & \\
\hline $\begin{array}{c}\% \text { wrong sign } \\
\text { contamination }\end{array}$ & $0.11 \pm 0.006$ & $0.14 \pm 0.004$ & $0.11 \pm 0.01$ & $0.11 \pm 0.002$ & $0.12 \pm 0.04$ \\
\hline
\end{tabular}

Table 6.1: Number of events in the CC selected neutrino sample for each of the categories is shown in the first row. The second row shows the percentage contribution that each sample makes to the total sample. The third and fourth rows show the percentage NC and wrong sign contamination in each sample.

these events tend to populate the low energy bins. The fractional NC contaminations are much higher in the antineutrino sample compared to the neutrino sample because NC events, which arise from both neutrinos and antineutrinos represent a fractionally larger contribution to the (smaller) antineutrino sample.

$\mathrm{CC}$ events in which the true sign of the muon track is mis-identified are referred to as "wrong sign" events. The wrong sign contamination in the neutrino sample is small $(\sim 0.15 \%)$, but is much higher in the antineutrino sample. The $\bar{v}_{\mu} \mathrm{CC}$ sample has a higher fractional contamination from wrong sign events due to the much larger $v_{\mu}$ component of the beam. This wrong sign contamination decreases with energy because as energy increases the fraction of $v_{\mu}$ in the beam decreases. Both NC and wrong sign contributions to the sample contamination result in events with higher average inelasticity than a pure antineutrino sample. The following additional cuts are applied to the $\bar{v}_{\mu} \mathrm{CC}$ sample to reduce this contamination:

1. Relative angle - Requires that tracks have a Relative Angle value less than 1.04 radians or greater than 5.24 radians. Relative angle is defined as the angle that the straight line joining 


\begin{tabular}{|c|c|c|c|c|c|}
\hline $\begin{array}{c}\text { Track Charge }>0 \\
\text { (selected antineutrinos) }\end{array}$ & $\begin{array}{c}\text { stopping } \\
\text { upstream }\end{array}$ & $\begin{array}{c}\text { exiting } \\
\text { upstream }\end{array}$ & $\begin{array}{c}\text { stopping } \\
\text { downstream }\end{array}$ & $\begin{array}{c}\text { exiting } \\
\text { downstream }\end{array}$ & Total \\
\hline \hline sample size & 15046 & 57636 & 6532 & 317819 & 397033 \\
\hline$\%$ contribution & 3.79 & 14.52 & 1.64 & 80.05 & 100 \\
\hline$\%$ NC & $49.43 \pm 0.57$ & $9.62 \pm 0.13$ & $3.75 \pm 0.24$ & $0.18 \pm 0.01$ & $3.48 \pm 0.03$ \\
contamination & $31.09 \pm 0.45$ & $17.90 \pm 0.18$ & $16.52 \pm 0.50$ & $4.85 \pm 0.04$ & $7.93 \pm 0.002$ \\
\hline $\begin{array}{c}\% \text { wrong sign } \\
\text { contamination }\end{array}$ & & & & & \\
\hline
\end{tabular}

Table 6.2: Number of events in the CC selected antineutrino sample for each of the categories is shown in the first row. The second row shows the percentage contribution that each sample makes to the total sample. The third and fourth rows show the percentage NC and wrong sign contamination in each sample.

the track end and extrapolated end of the track in absence of a magnetic field makes with the line passing through the coil hole and the vertex. Fig. 6.8 shows an illustration of this variable. The standard magnetic field polarity focuses the $\mu^{-}$tracks and defocuses the $\mu^{+}$tracks, as a result the relative angle distribution for $\mu^{-}$s peaks around $\pi$ radians and for $\mu^{+}$s it peaks at 0 and $2 \pi$ radians. Fig. 6.9 shows the distribution of relative angle for neutrino and antineutrino samples. The cut is determined by plotting the product of efficiency and purity as a function of the difference of relative angle and $\pi$ and choosing the value at which this product is maximum. This value is determined to be 2.1 radians, which when subtracted or added to $\pi$ gives the two limits on relative angle.

2. Number of hit planes - Keeps events in which the difference in the number of hit planes in the track in the two views is less than 5. Events which are rejected by this cut usually enter the uninstrumented region in one view yielding an unreliable determination of the charge sign. Fig. 6.10 shows the fractional $\mathrm{CC}$ contamination as a function of the difference in the number of hit planes in the track in the two views. The $\mathrm{CC}$ contamination can be reduced by restricting the plane difference in the two views to be less than 5 . 


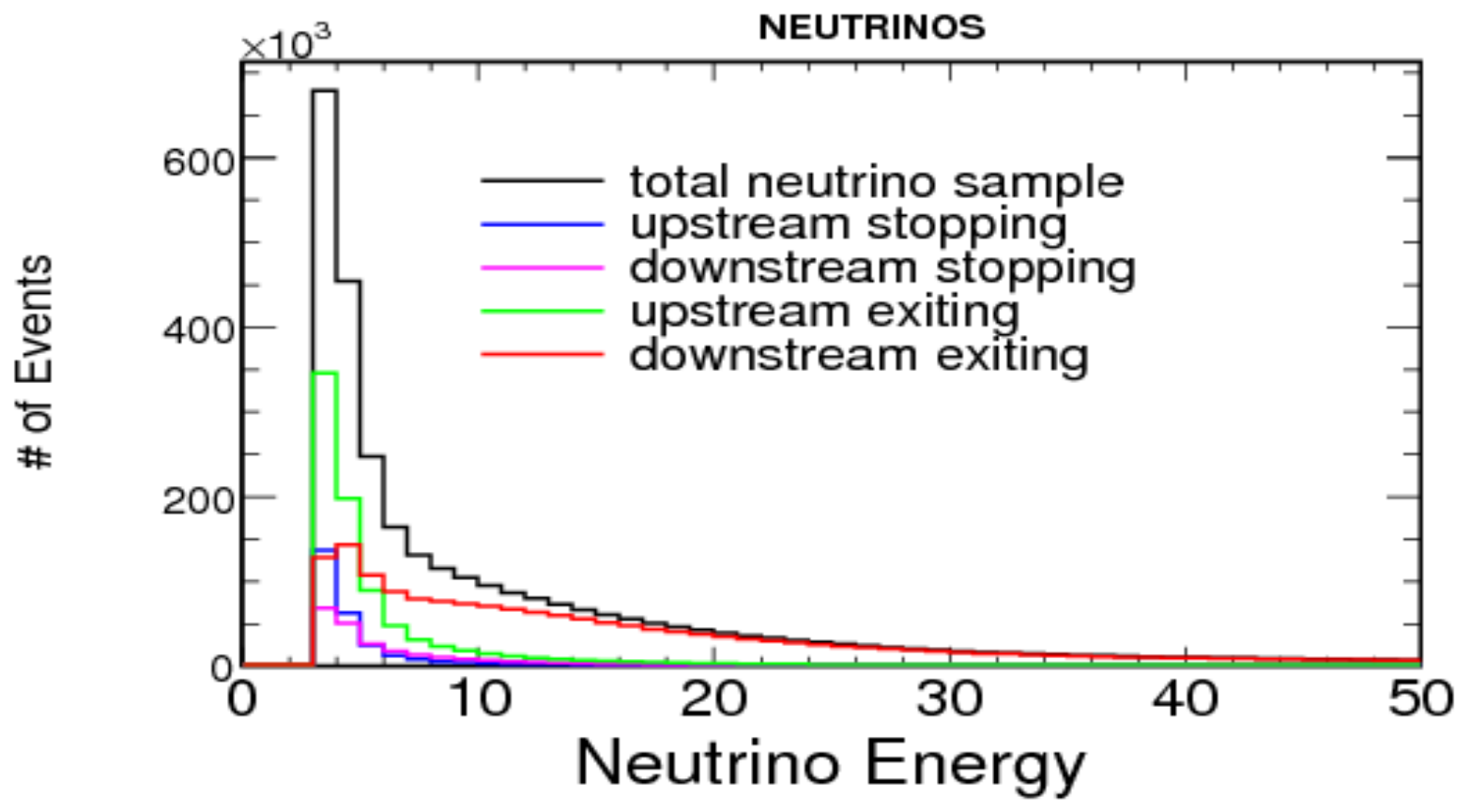

(a)

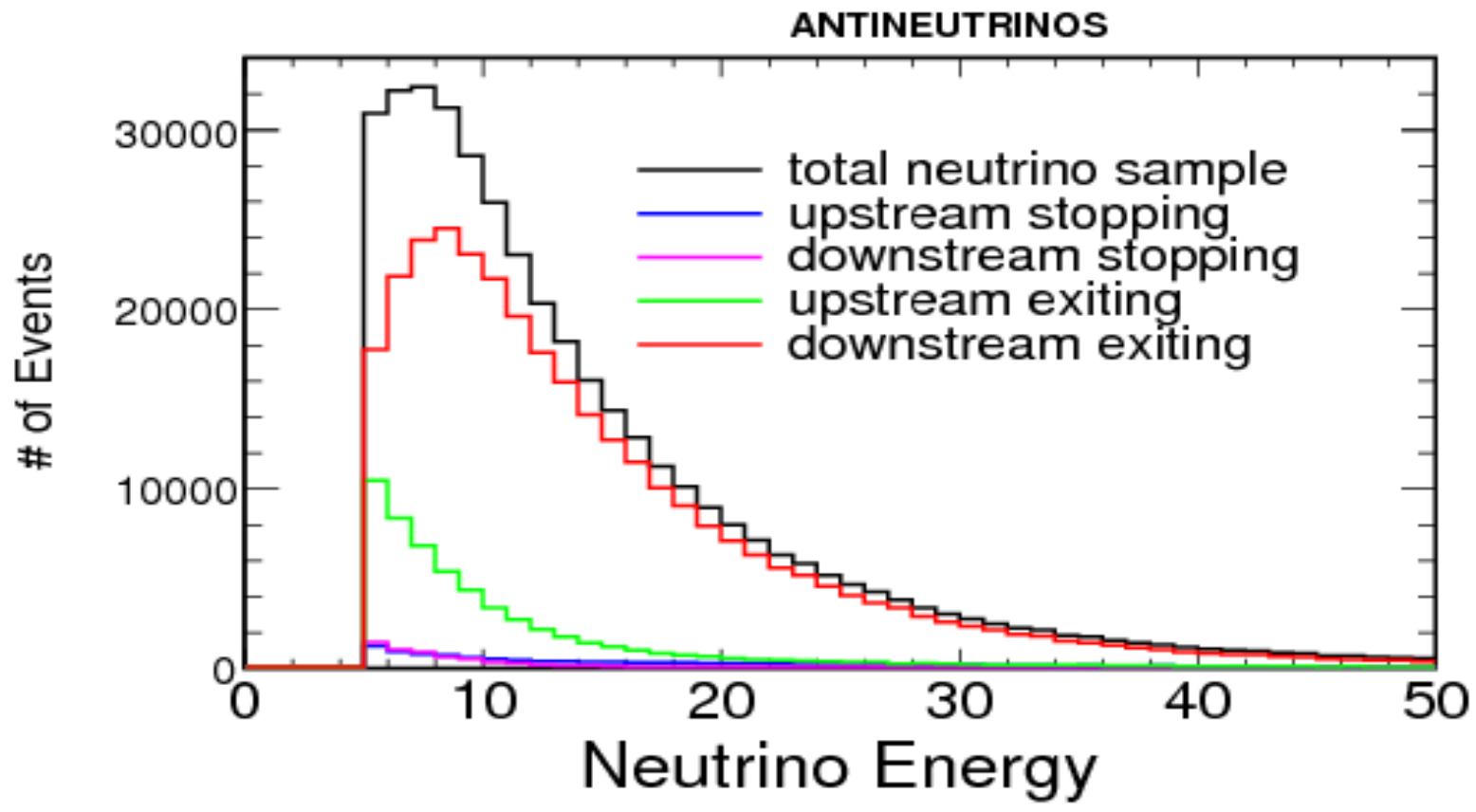

(b)

Figure 6.6: Energy distribution for neutrino and antineutrino $\mathrm{CC}$ subsamples. 


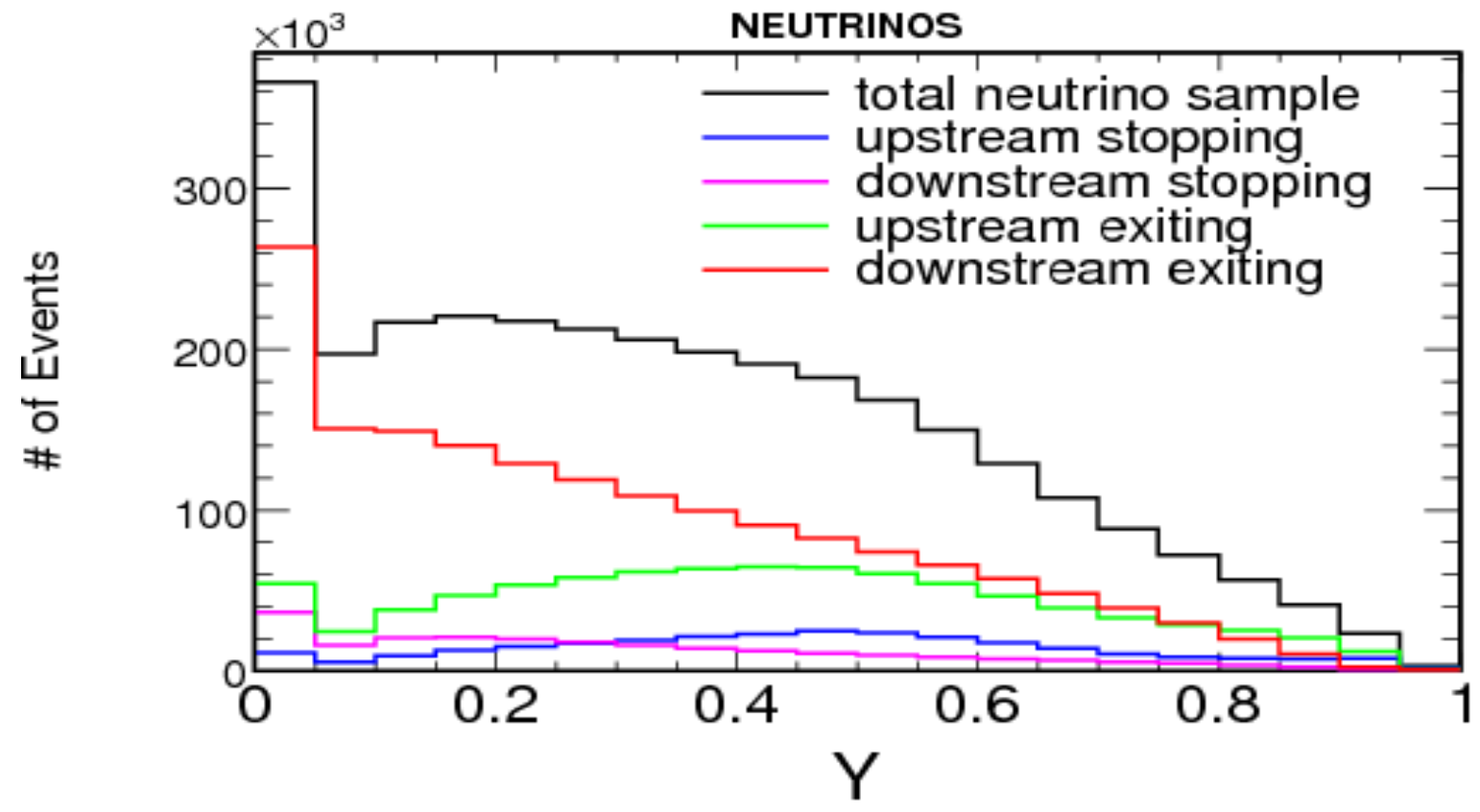

(a)

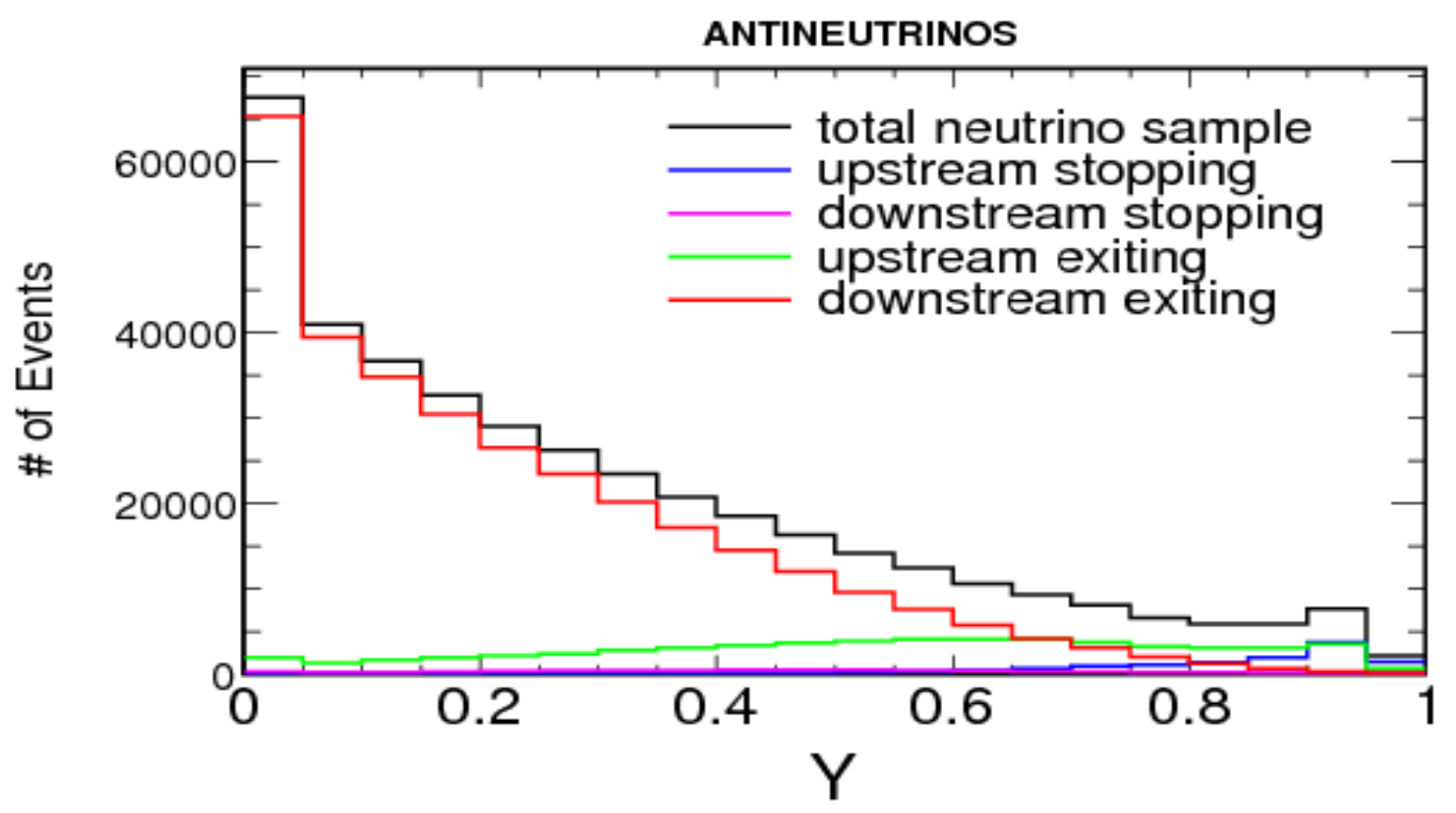

(b)

Figure 6.7: Inelasticity distribution for neutrino and antineutrino CC subsamples. 
3. Downstream exiting tracks - Include only events with tracks that exit the detector in the downstream region (see Fig. 2). As can be seen from Table 6.2, the rejected samples have unacceptably high contamination from NC and wrong sign events because these samples have lower energy and hence a larger overlap with the $v_{\mu}$ sample.

After applying the additional cuts, the percentage NC contamination is $0.2 \%$ and wrong sign contamination is $1.1 \%$ in the antineutrino sample. These contaminations are eventually subtracted based on MC simulation (see section 7.1).

\subsection{EFFECT OF CUTS}

Fig. 6.11 shows the effect of the selection criteria as a function of neutrino energy for the neutrino and antineutrino sample. The minimum track energy cut has the largest effect resulting in an approximately $20 \%$ loss in the $v_{\mu}$ sample and $40 \%$ in the $\bar{v}_{\mu}$ sample. Since the track energy cut removes NC contamination it has a larger fractional effect on the antineutrino sample. The cut that requires the error on $\mathrm{q} / \mathrm{p}$ (referred to as the eqp/qp cut in the figure) to be less than $30 \%$, has a dip at high energy for the antineutrino sample. This is produced by tracks that exit the detector through the side rather than the end of the detector. Table 6.3 shows the effect of the selection cuts on the neutrino and the antineutrino reconstructed samples. The final $v_{\mu}$ sample has $1.94 \times 10^{6}$ events and the $\bar{v}_{\mu}$ sample has $1.59 \times 10^{5}$ events.

\subsection{ACCEPTANCE CORRECTION}

The acceptance correction $A_{c c}^{M C}(E)$ is defined as the ratio of the number of generated $\mathrm{MC}\left(N^{G E N}(E)\right)$ events in a bin within the true fiducial volume to the reconstructed $\mathrm{CC}$ selected $\mathrm{MC}\left(N^{R E C O M C}(E)\right)$ events within that fiducial volume,

$$
\operatorname{Acc}^{M C}(E)=\frac{N^{G E N}(E)}{N^{R E C O M C}(E)} .
$$




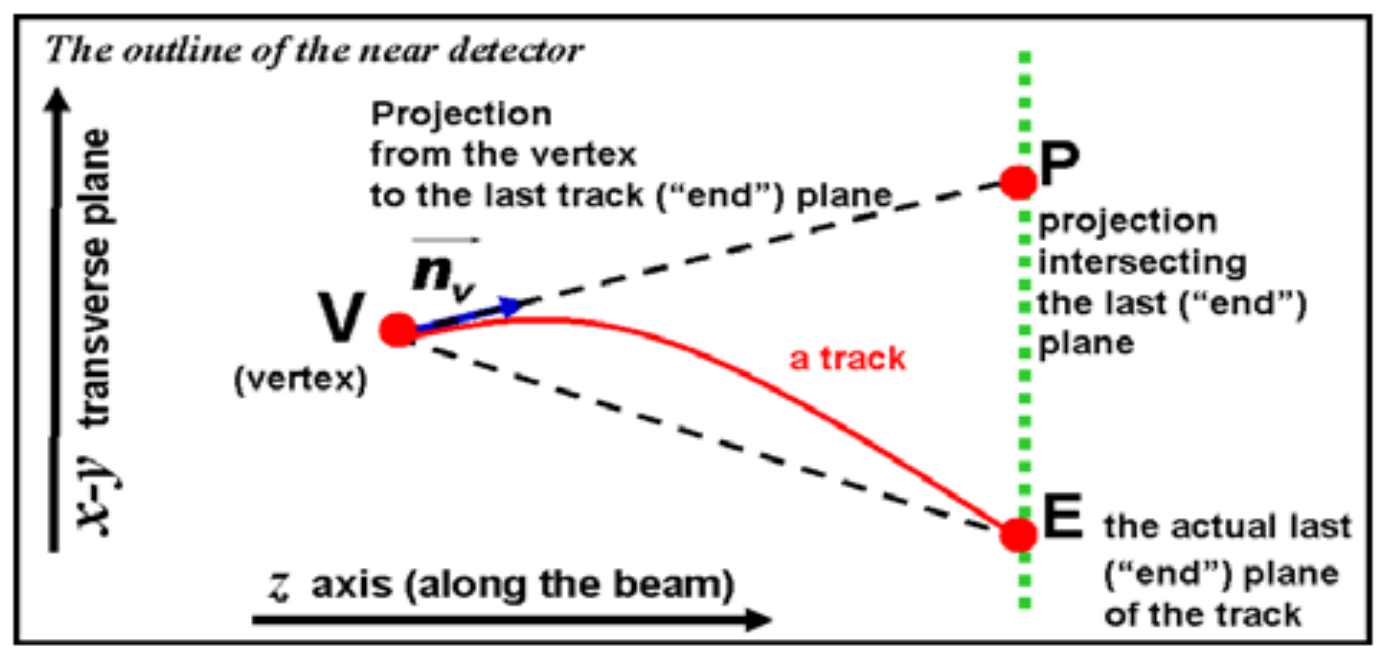

(a)

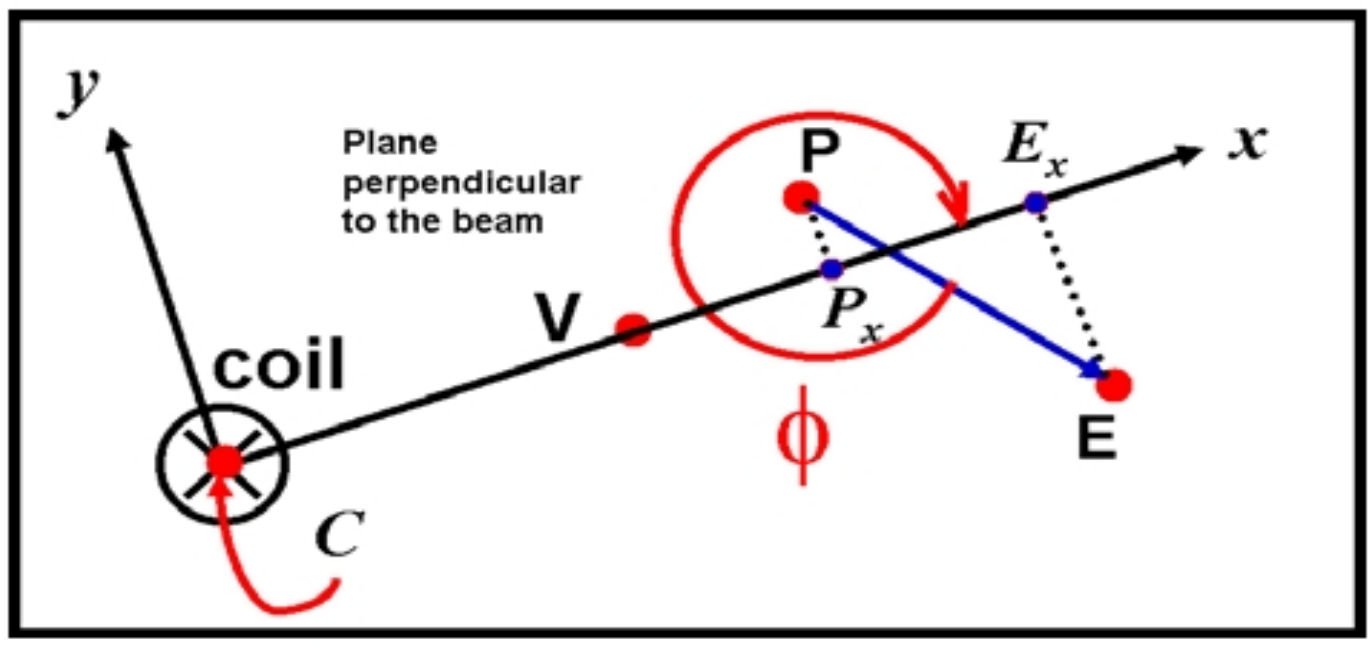

Figure 6.8: An illustration defining the Relative Angle variable[52]. Fig.(a) shows a straight line $\left(\overrightarrow{n_{V}}\right)$ along the initial direction of the track that projects the track vertex $\mathrm{V}$ to the point $\mathrm{P}$ on the last hit plane. E is the position of the actual track end. Fig.(b) shows the same track in the $x-y$ plane where the points V,P and E are shown relative to each other. For each track, a local Cartesian co-ordinate is constructed that has the line joining the coil hole $(\mathrm{C})$ and the point $\mathrm{V}$ as its $\mathrm{x}$-axis and a line orthogonal to it as the y-axis. 


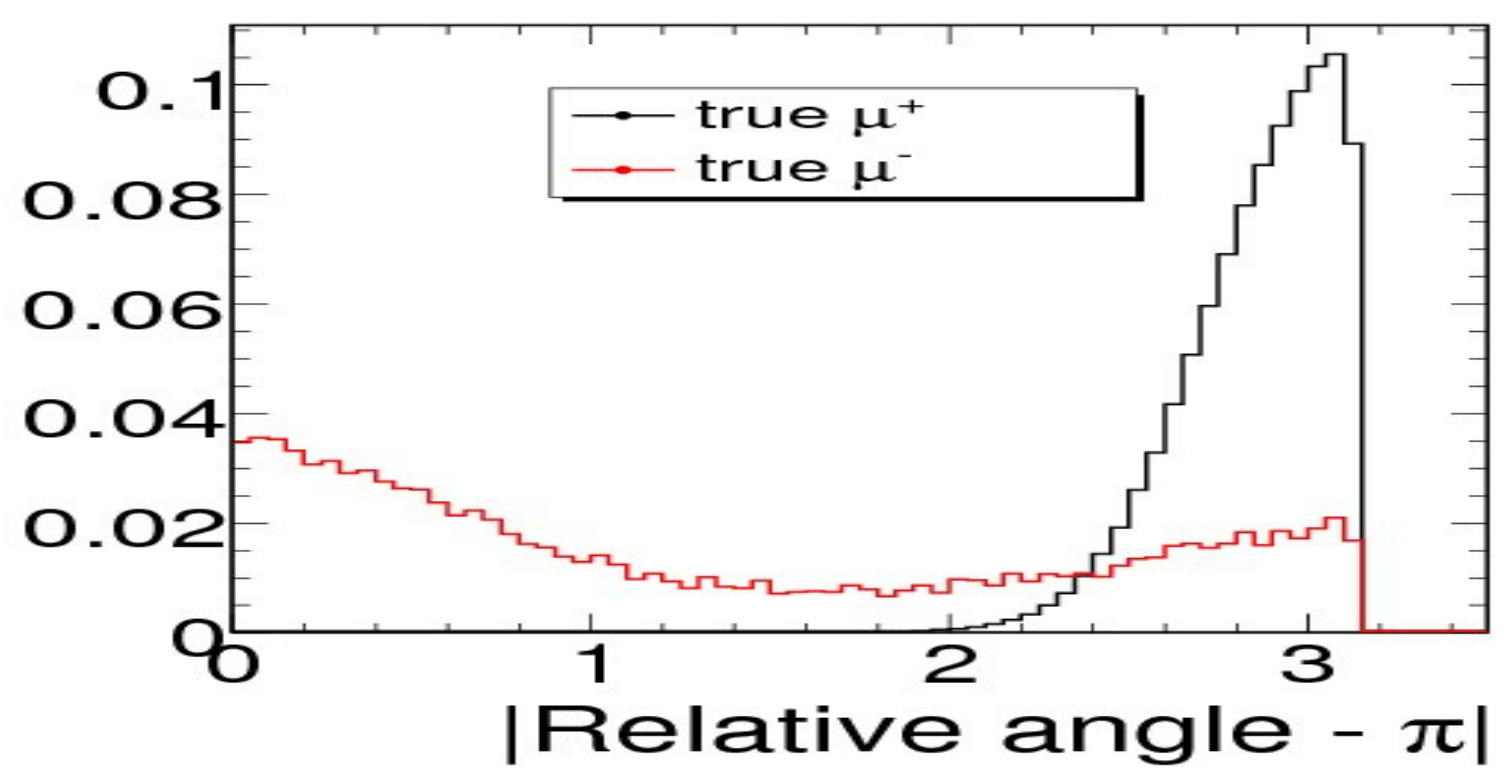

(a)

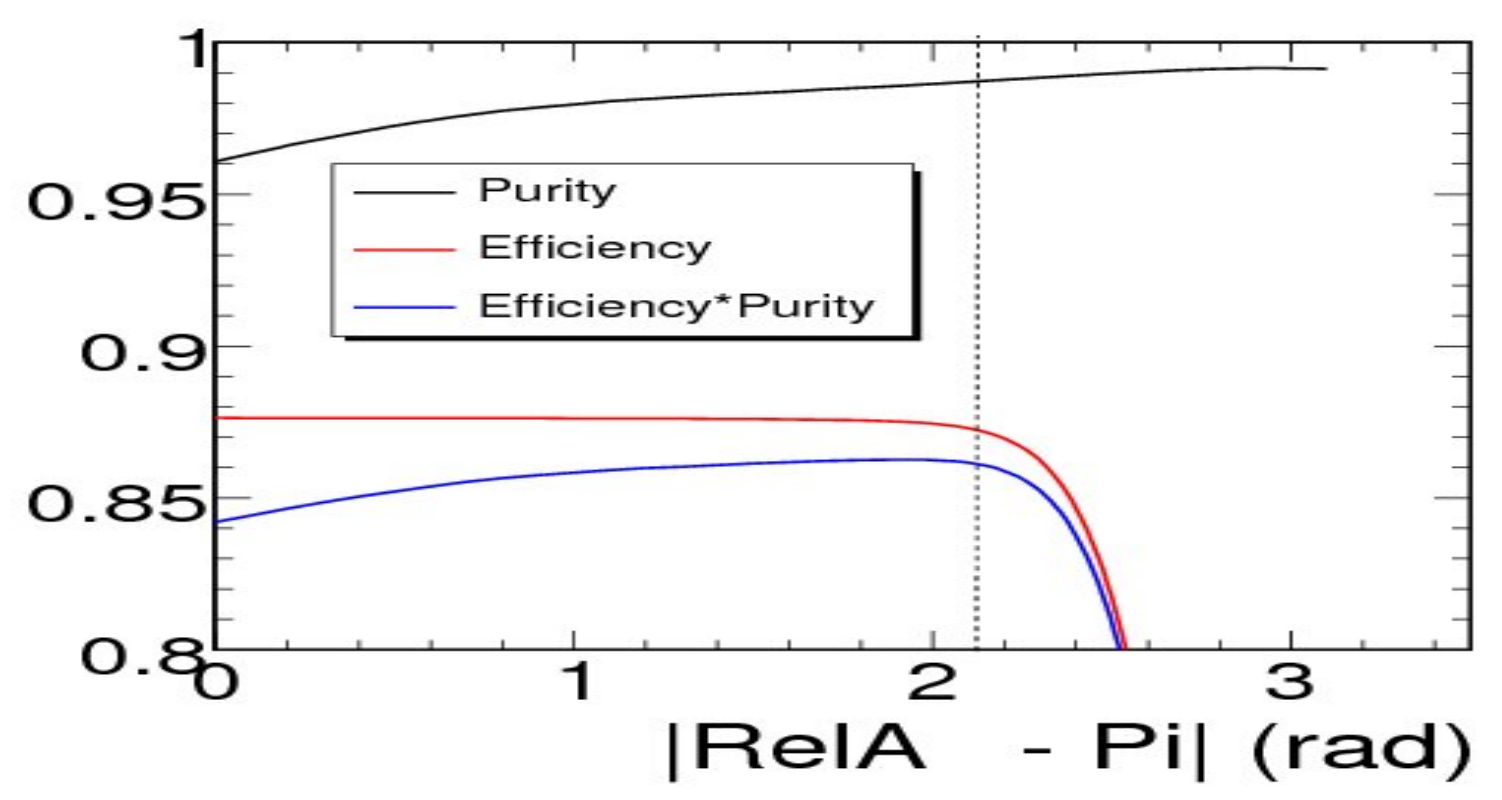

(b)

Figure 6.9: Distribution of Irelative angle- $\pi$ I in the reconstructed antineutrino sample(a). The black line shows the distribution for true neutrinos and the red line shows the same for true antineutrinos. Plot on the bottom(b) shows the efficiency, purity and product of efficiency and purity as a function of |relative angle- $\pi$ |. 


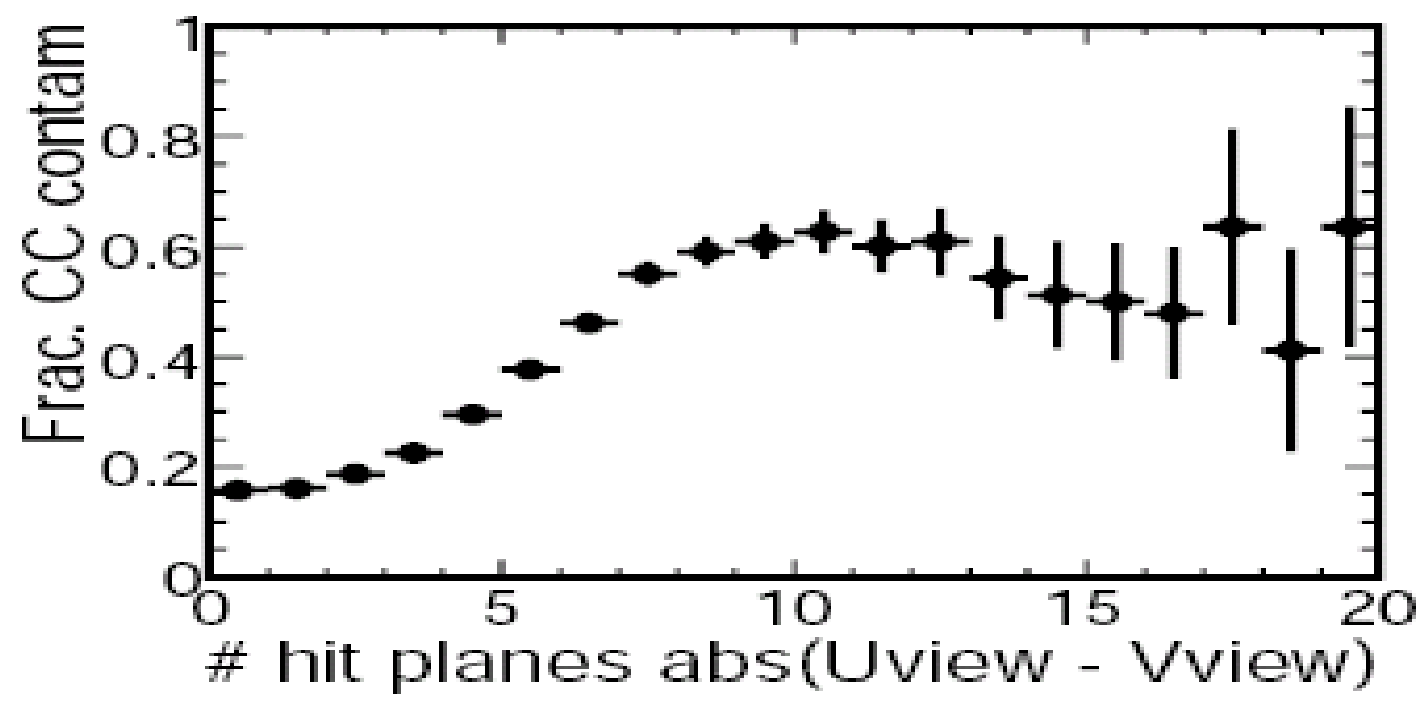

Figure 6.10: Fractional CC contamination for the reconstructed antineutrino sample as a function of the difference in the number of hit planes in the $\mathrm{U}$ and $\mathrm{V}$ view. 


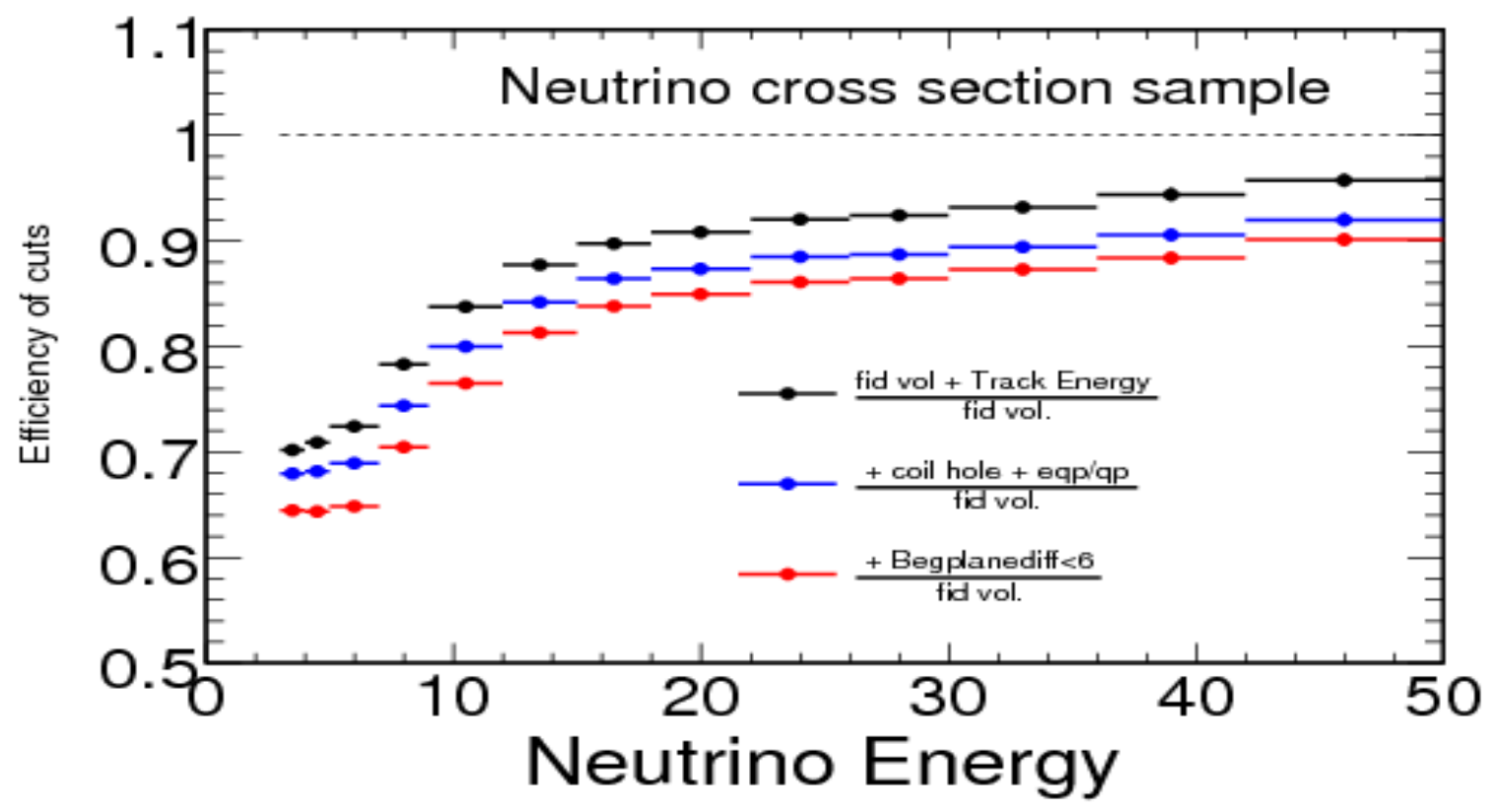

(a)

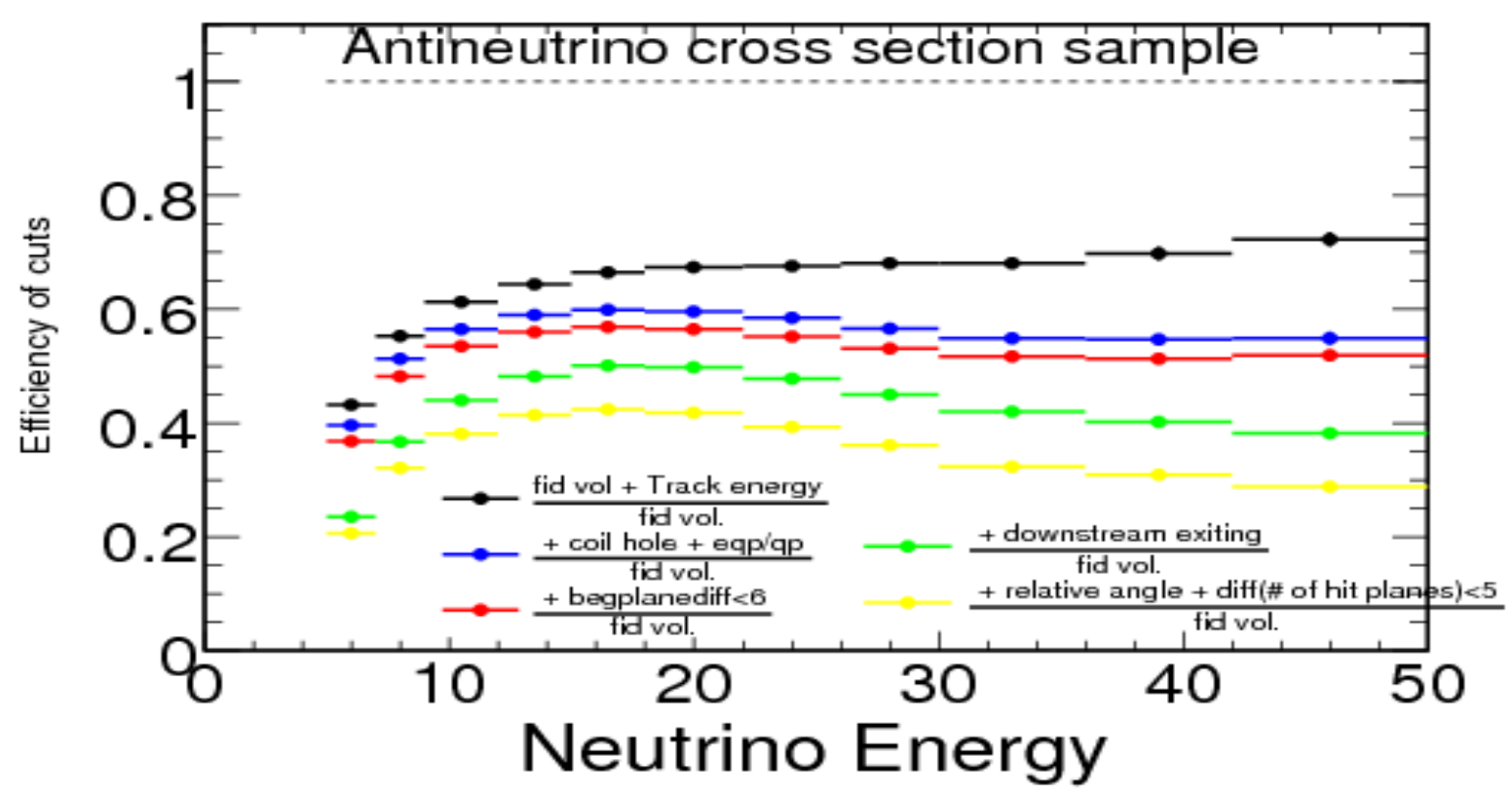

(b)

Figure 6.11: Efficiency of selection cuts for neutrino(a) and antineutrino(b) CC cross section sample. Each line shows the effect of the cuts in a cumulative manner. 


\begin{tabular}{|c|c|c|}
\hline Selection Criterion & Track Charge $<0(\%$ loss $)$ & Track Charge $>0(\%$ loss $)$ \\
\hline \hline Track in fiducial volume & 3608572 & 841986 \\
\hline $3<E_{v}<50 \mathrm{GeV}\left(5<E_{v}<50 \mathrm{GeV}\right.$ for $\left.\bar{v}_{\mu} \mathrm{s}\right)$ & $2680994(25.7 \%)$ & $474763(43.6 \%)$ \\
\hline$E_{\mu}>1.5 \mathrm{GeV}$ & $2116618(21.1 \%)$ & $276936(41.7 \%)$ \\
\hline Track Quality Cuts & $1941019(8.3 \%)$ & $235024(15.1 \%)$ \\
\hline Additional $\bar{v}_{\mu}$ cuts & - & $159880(32 \%)$ \\
\hline
\end{tabular}

Table 6.3: Effect of the selection criteria on the negatively(left) and positively(right) charged reconstructed samples. The numbers in bracket show the percentage of events lost due to the cut in the previous row.

Fig. 6.12 shows the acceptance correction for neutrino and antineutrino CC sample. The acceptance correction value at high energy is about 1.25 and increases at low energy due to energy threshold effects. The dip in the neutrino acceptance correction around $8 \mathrm{GeV}$ is caused by the overlap of different subsamples and detector geometry. Fig. 6.13 shows the reciprocal of acceptance correction for the total neutrino cross section sample and the contribution from different samples.

The dip is also caused by $\mu^{-}$tracks that are focused in the detector and have a high probability of passing through the coil hole. Track fitting for many of these tracks fail and hence they are rejected by the $\mathrm{CC}$ selection. Fig. 6.14 shows the track end y versus end $\mathrm{x}$ distribution for reconstructed neutrino tracks. The tracks that fail the fitter(shown by the bottom plot) have crossed the coil hole, which is the area on the $x<0 \mathrm{~m}$ region. Fig. 6.15(a) shows the ratio of tracks that are rejected due to fitting failure in data and $\mathrm{MC}$ event sample as a function of track end $\mathrm{z}$ vertex. From Fig.6.15(b) we can see that all the rejected tracks are neutrinos. There is a larger effect $(\sim 10 \%)$ for longer tracks because they are more likely to bend in the magnetic field and pass through the coil hole. The 5\% discrepancy in data and simulation is caused mainly due to the mis-modeling of the coil hole. To check the impact of this effect on the analysis, a special data set was collected with magnetic field reversed in the detector and is described in section 6.6. 


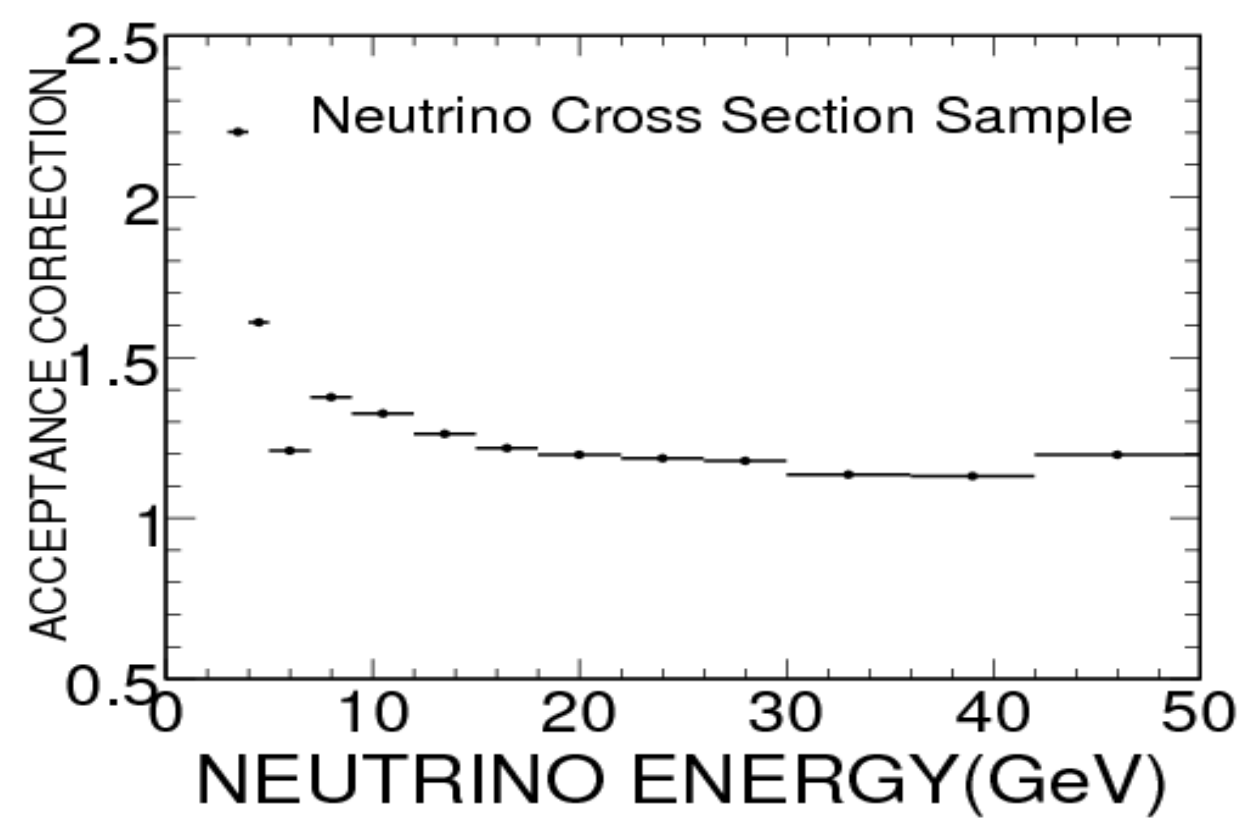

(a)

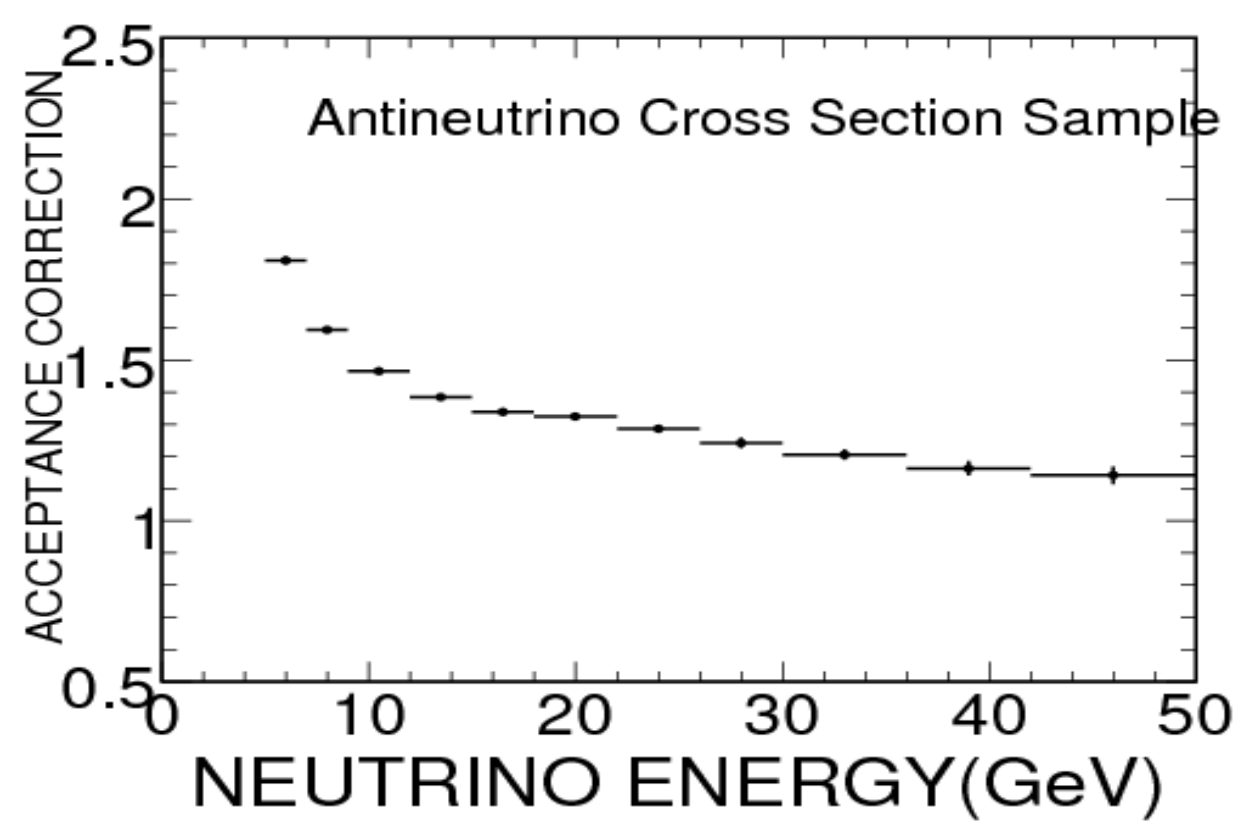

(b)

Figure 6.12: Acceptance correction from MC simulation for neutrino(a) and antineutrino(b) CC selected sample. 


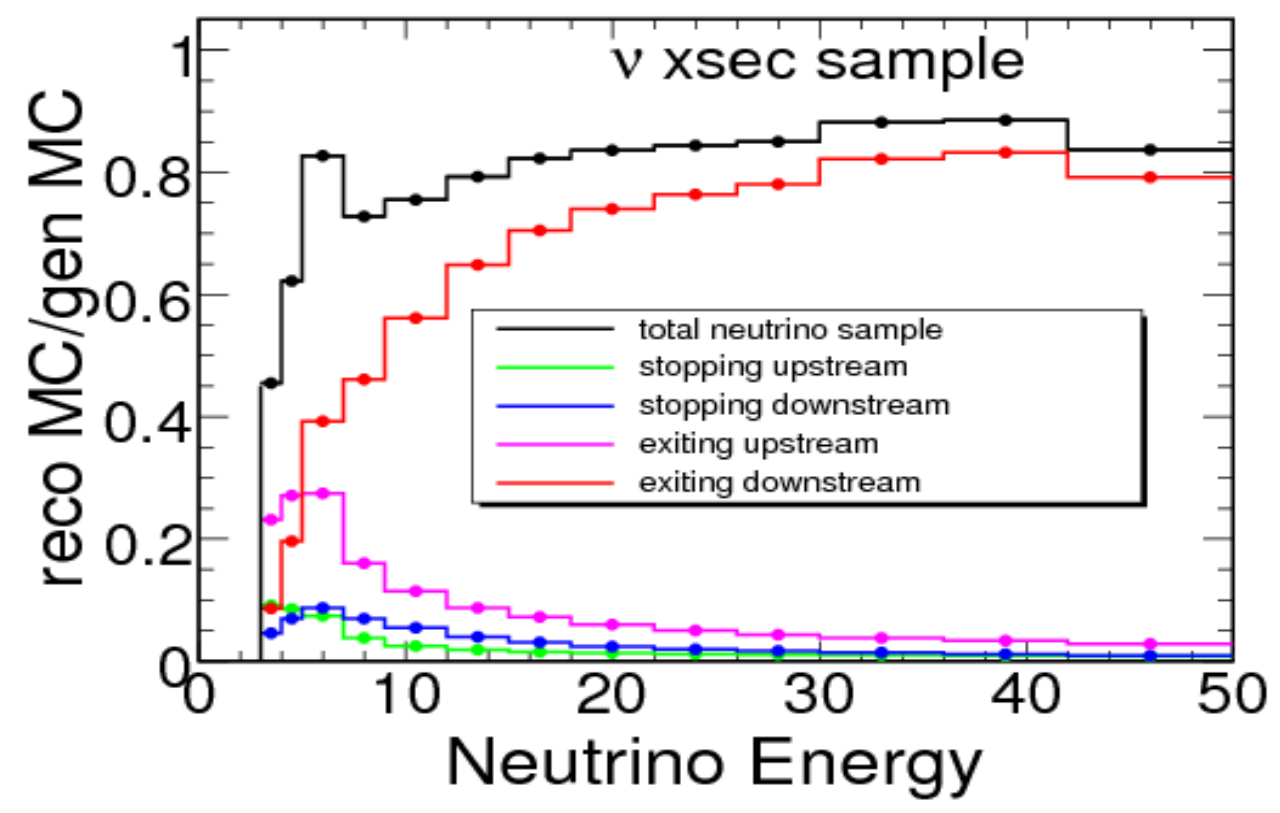

Figure 6.13: Reciprocal of acceptance correction for the selected charged current neutrino sample and the sub-samples(shown by the colored lines). 


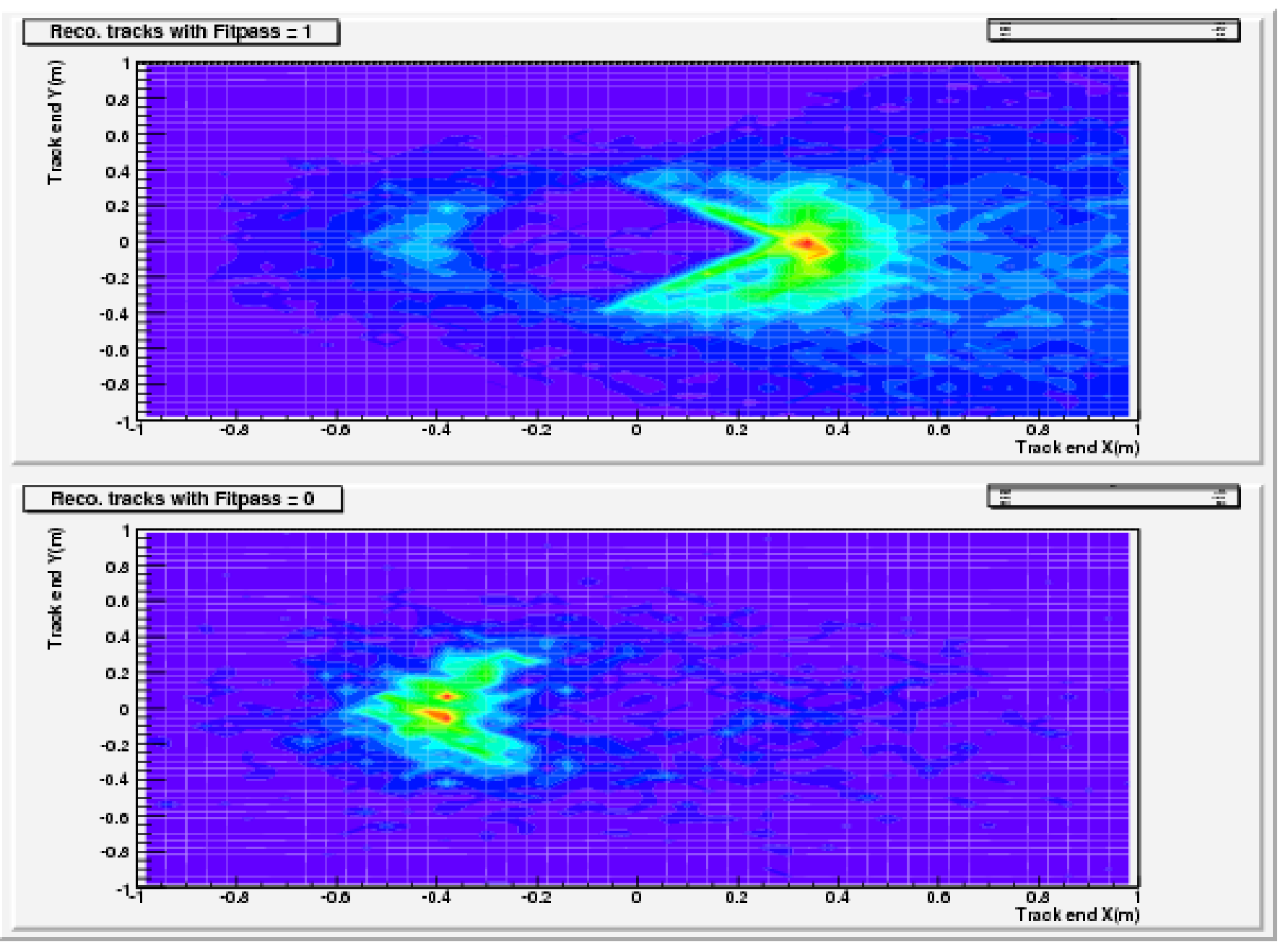

Figure 6.14: Track end y versus end $\mathrm{x}$ for reconstructed neutrino sample that pass track fitting(top) and that fail track fitting(b). 


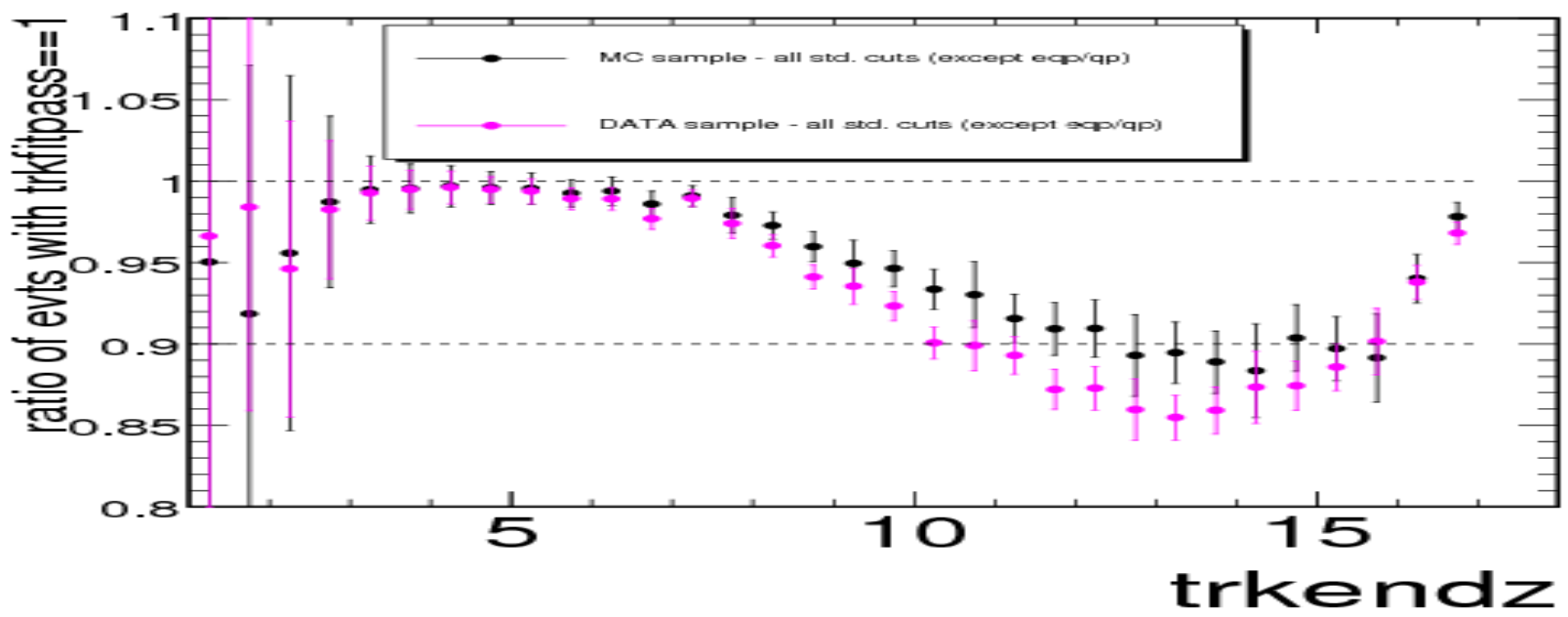

(a)

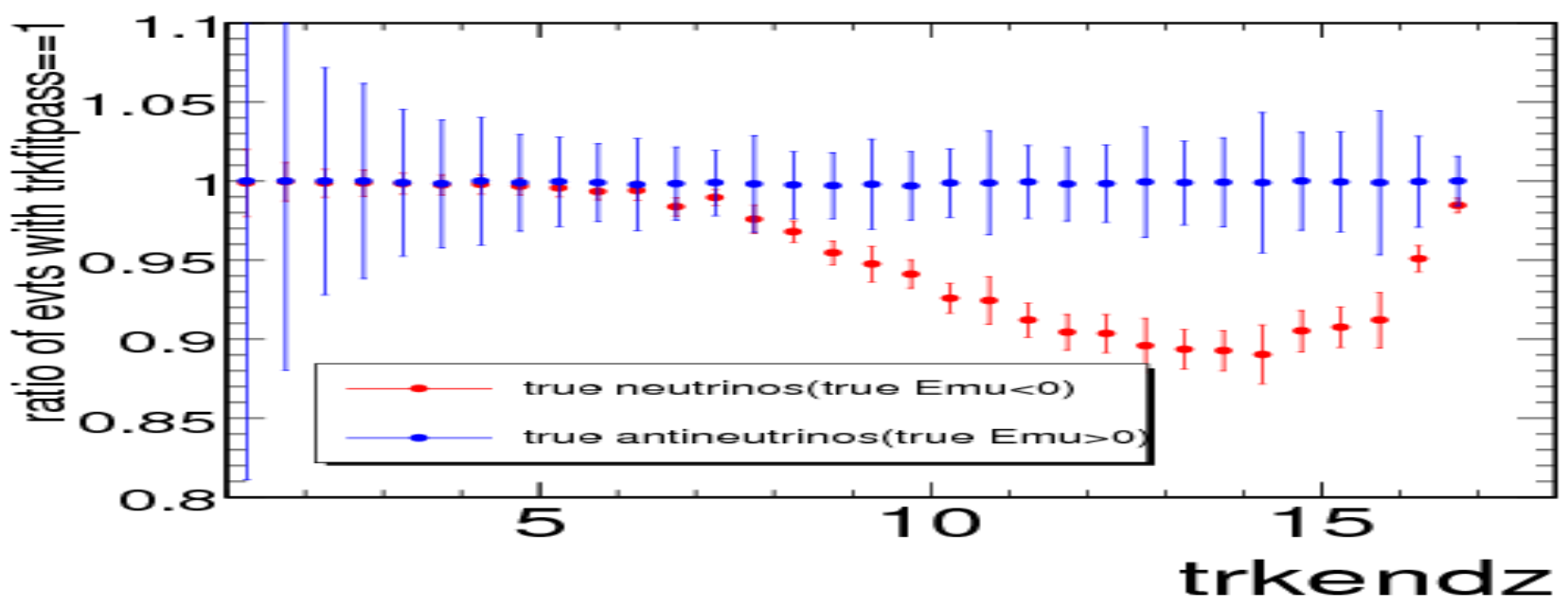

(b)

Figure 6.15: Effect of track fitter failure on total CC selected sample(both neutrino and antineutrino) as a function of track end $\mathrm{z}(\mathrm{m})$. The top plot(a) shows data and $\mathrm{MC}$ simulation comparison and the bottom plot(b) shows the MC event sample broken down into true neutrinos and true antineutrinos. 


\subsection{DATA AND MC SIMULATION COMPARISON}

This section presents the data and MC simulation comparison of track vertex, track end, $E_{\mu}, E_{s h w}$, $\theta_{\mu}$ and $y$. The plots are normalized to PoTs. Once we extract the flux (section 7.2), the MC event sample is reweighted with the extracted flux to remove any effects that the default input flux might have had on the analysis. The flux reweighting has been applied to all the MC distributions shown here.

Fig. 6.16 shows the $\mathrm{x}, \mathrm{y}$ and $\mathrm{z}$ track vertex distribution for the data and $\mathrm{MC}$ event sample. The antineutrino MC sample shows good agreement with the data. The neutrino sample has an offset of about $2 \%$ in the $\mathrm{y}$ and $\mathrm{z}$ vertex distribution. The data over $\mathrm{MC}$ ratio for $\mathrm{x}$ vertex has a slope of $\sim 2 \% /$ meter. This behavior is partially caused by the tracks that end in the coil hole and fail the fitter (as explained in section 6.4).

Fig. 6.17 shows the track end radial and track end $\mathrm{z}$ distribution for the data and MC event sample. The track end radial distribution for neutrinos peak around the coil hole because the $\mu^{-} s$ are focused. For antineutrinos, the distribution peaks at the edge of the detector because the $\mu^{+} s$ are defocused. The end $\mathrm{z}$ distribution shows that for both neutrinos and antineutrinos, a large fraction of the tracks exit the detector through the end $(\mathrm{z}>15 \mathrm{~m})$. A general feature of all the plots is data and MC discrepancy of the order of $\sim 20 \%$ at the edge of the detector because the last plane of an exiting track is not modeled well by the MC simulation. This doesn't affect the analysis as the mis-modeling effect is present in only the last plane of a long track. This could affect the momentum measured from range, but since these tracks are exiting we use momentum measured from curvature. A discrepancy of about $20 \%$ is also present in the radial track end distribution for neutrinos around the coil hole. The neutrino radial distribution agrees at the level of $5 \%$ between data and $\mathrm{MC}$ in other regions. Antineutrinos don't get affected by coil hole modeling and show an overall good agreement $(\sim 5 \%)$ between data and MC simulation. The coil hole mismodeling affects the track vertex and the track end distributions. The data collected with the reversed magnetic field polarity will help in evaluating the effect of the coil hole on the analysis as described in section 6.6.

Fig. 6.18 shows a comparison of the measured kinematic variables $E_{\mu}, E_{s h w}$ and $\theta_{\mu}$ (before and after flux reweighting). Agreement between data and MC shows a significant improvement 
after the flux reweighting function is applied. The $\chi^{2} /$ dof for all the distributions are less than 1. Fig. 6.19 shows the comparison of data and MC simulation for $y$ distribution. The neutrino agreement improves after applying the flux reweighting function. Reweighting has a small effect on the antineutrino MC $y$ distribution which shows disagreement in the low $y(<0.3)$ region. This can be caused by the cross section model uncertainty from the $M_{A, Q E L}$ and $M_{A, R E S}$ parameters which are important at low $y$.

\subsection{REVERSE FIELD DATA}

The acceptance correction can be affected by our modeling of the detector geometry, alignment, and the magnetic field. This is particularly important for the focused (neutrino) sample because focused tracks can pass through the coil hole region where the field is uncertain and the track reconstruction can fail (as described in section 6.3). To understand how well this is modeled, a separate data set was collected with the polarity of the detector magnetic field reversed. As a result, the tracks spend more of their time in a different region of the detector. For example, the $\mu^{-} \mathrm{s}$ are defocused and bend away from the coil hole.

The dataset was collected in April 2007 and April-June 2008 with reversed magnetic field at the Near Detector with an exposure corresponding to $4.2 \times 10^{19}$ PoTs. The analysis was repeated with this dataset to obtain an estimate of the systematic uncertainty (see section 7.5) from acceptance correction. This section describes the data and MC event sample collected with the reversed field polarity.

Fig. 6.20 shows the comparison of reciprocal of acceptance correction between forward field and reverse field $\mathrm{MC}$ simulation. For neutrinos around $10 \mathrm{GeV}$, the reverse field sample has a larger number of events as compared to the forward field. This is expected because the tracks are not getting rejected due to the effect of the coil hole. As energy increases, there are fewer events in the neutrino reverse field sample because a much larger number of $\mu^{-}$s are exiting the detector. The trend in the antineutrino sample is opposite, with fewer events being accepted at lower energy compared to the forward field(because of the coil hole effect).

Fig. 6.21 shows the data and MC track vertex distribution for neutrinos and antineutrinos. The 

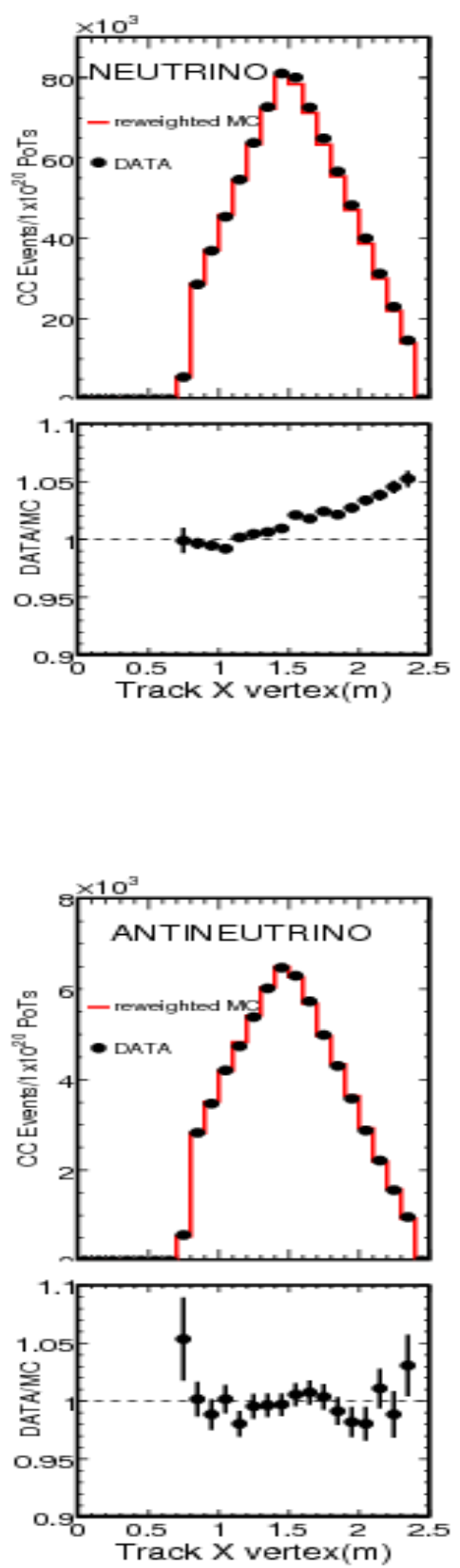
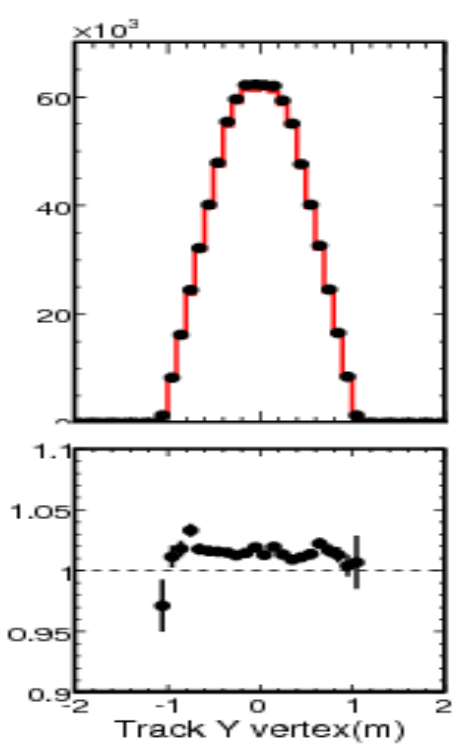

(a)
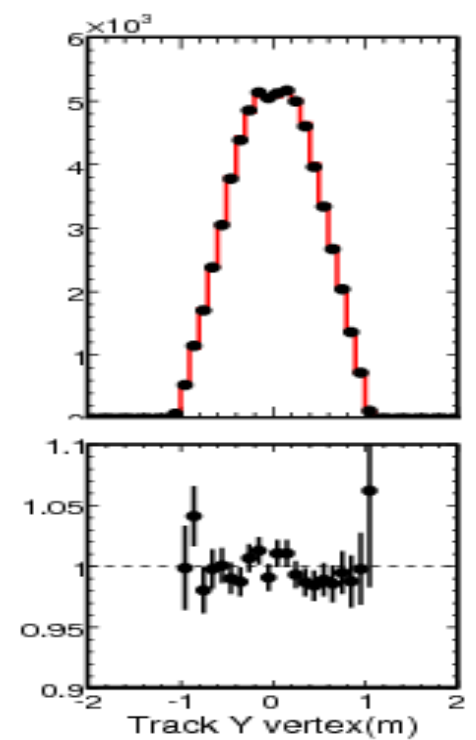
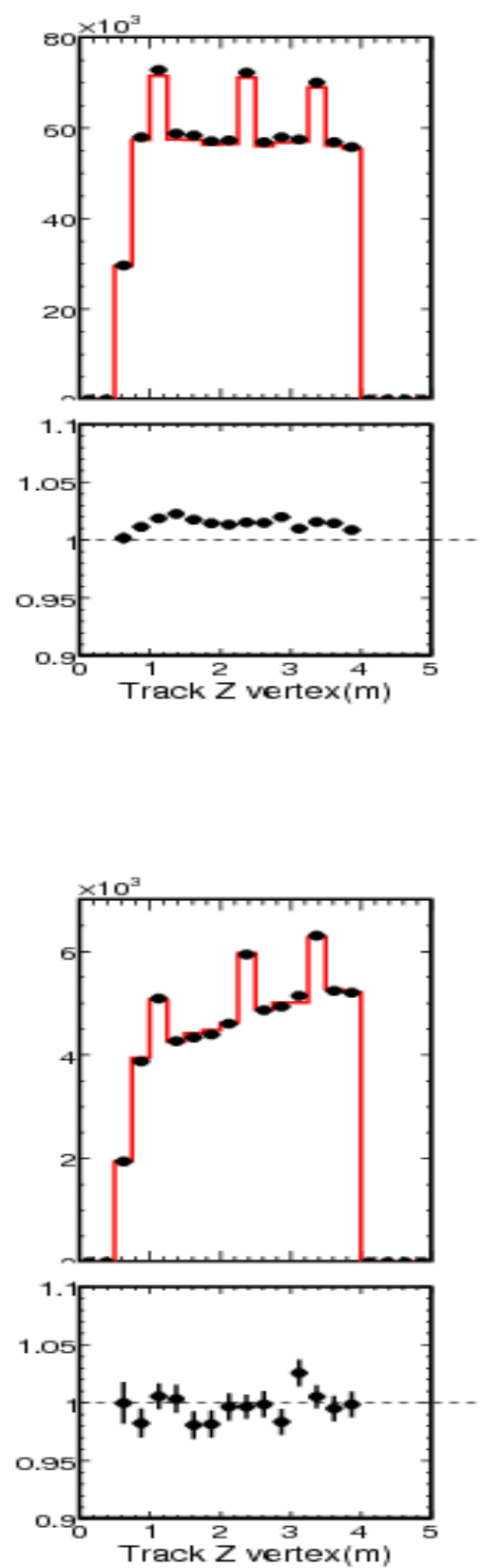

(b)

Figure 6.16: Comparison of data and $\mathrm{MC}$ simulation distribution for track beginning $\mathrm{x}, \mathrm{y}$ and $\mathrm{z}$ for neutrinos(a) and antineutrinos(b) in the forward field mode. The points show data and red curve shows MC model after applying flux reweighting. The ratio of data to MC is shown below each distribution. The error bars show the statistical error only. 

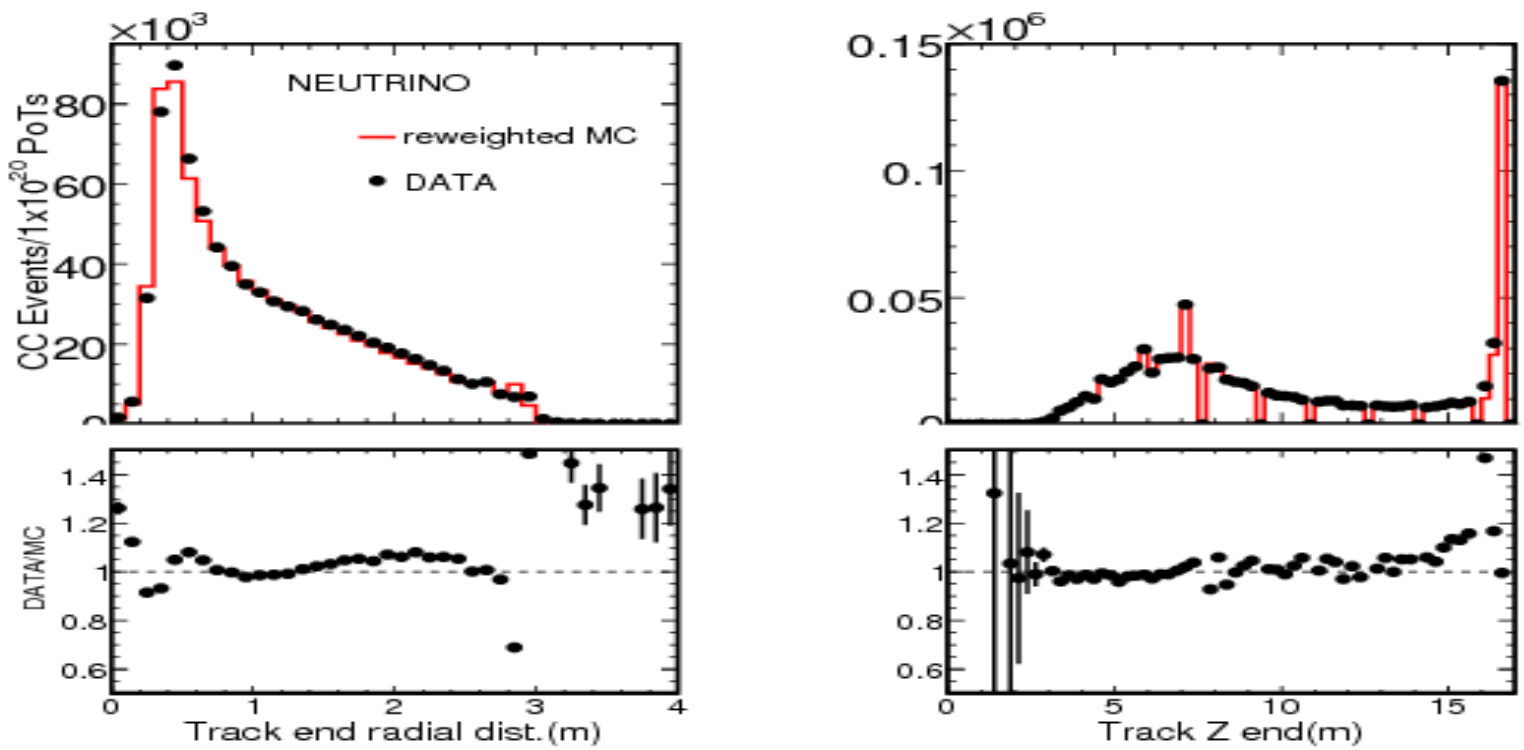

(a)
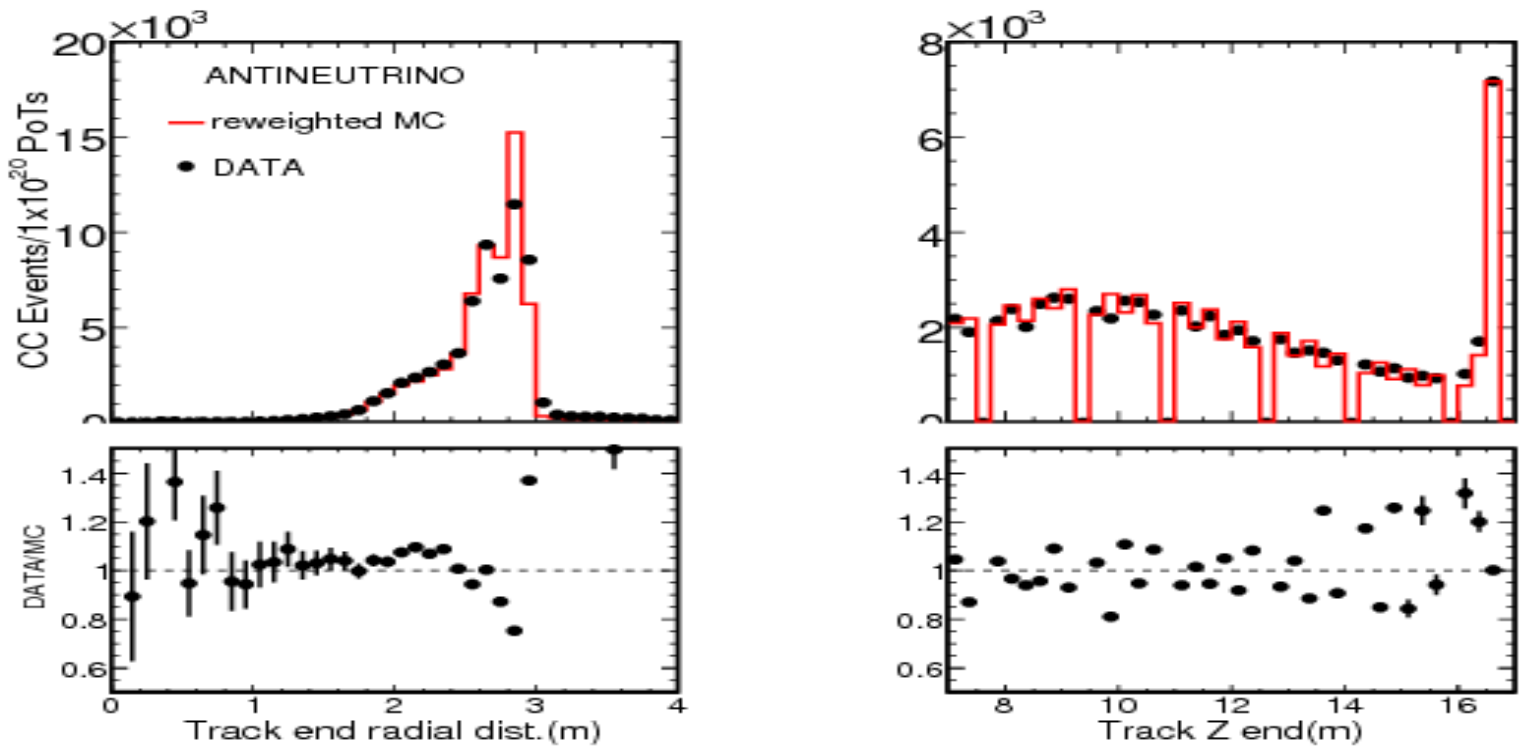

(b)

Figure 6.17: Comparison of data and MC simulation distribution for track end radial and track end $\mathrm{z}$ for neutrinos(a) and antineutrinos(b) in the forward field mode. The points show data and red curve shows MC model after applying flux reweighting. The ratio of data to $\mathrm{MC}$ is shown below each distribution. The error bars show the statistical error only. 

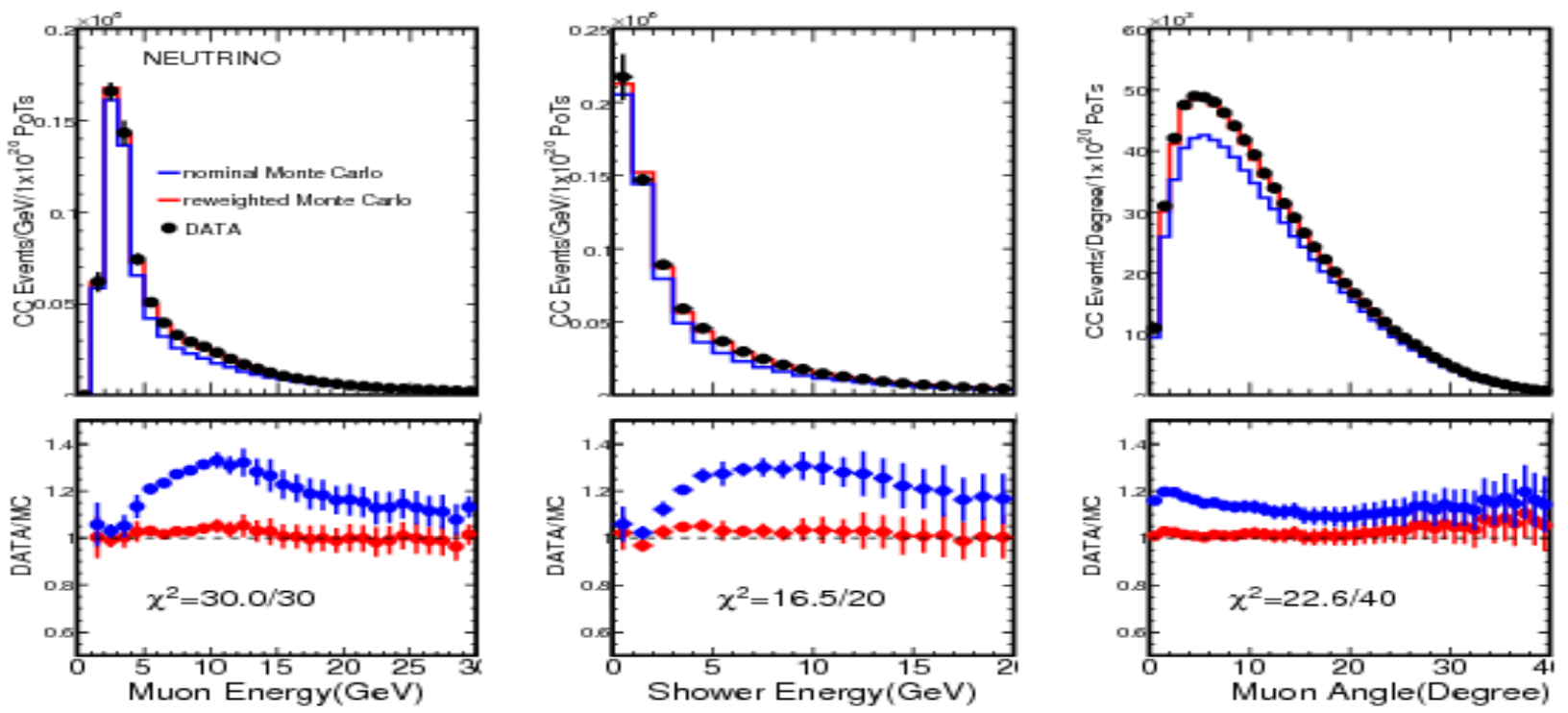

(a)
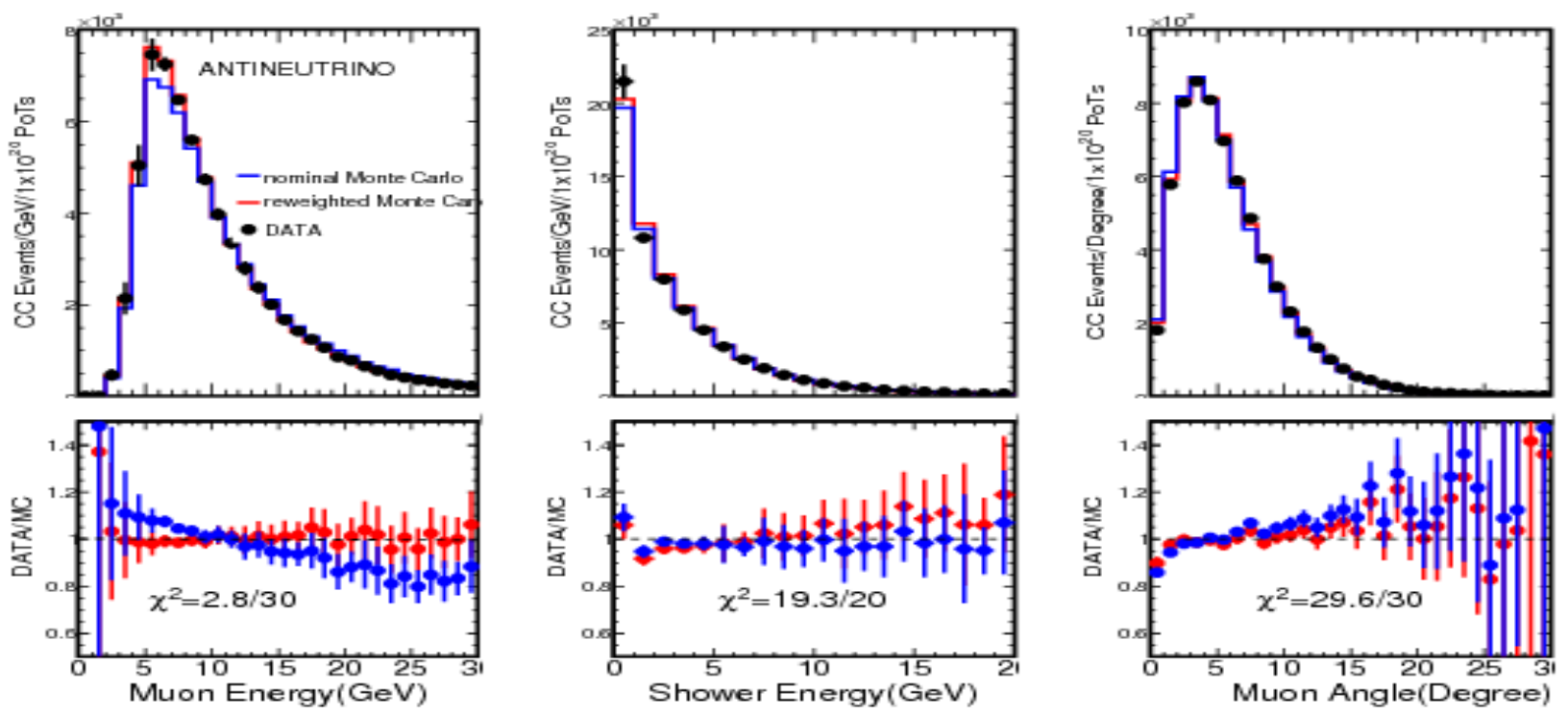

(b)

Figure 6.18: Comparison of data and MC simulation distribution for the kinematic variables $E_{\mu}$, $E_{s h w}$ and $\theta_{\mu}$ for neutrinos(a) and antineutrinos(b) in the forward field mode. The points show data, the blue curve shows nominal MC model and the red curve shows MC model after applying flux reweighting. The ratio of data to MC is shown below each distribution. The error bars show the statistical and systematic uncertainties added in quadrature. 

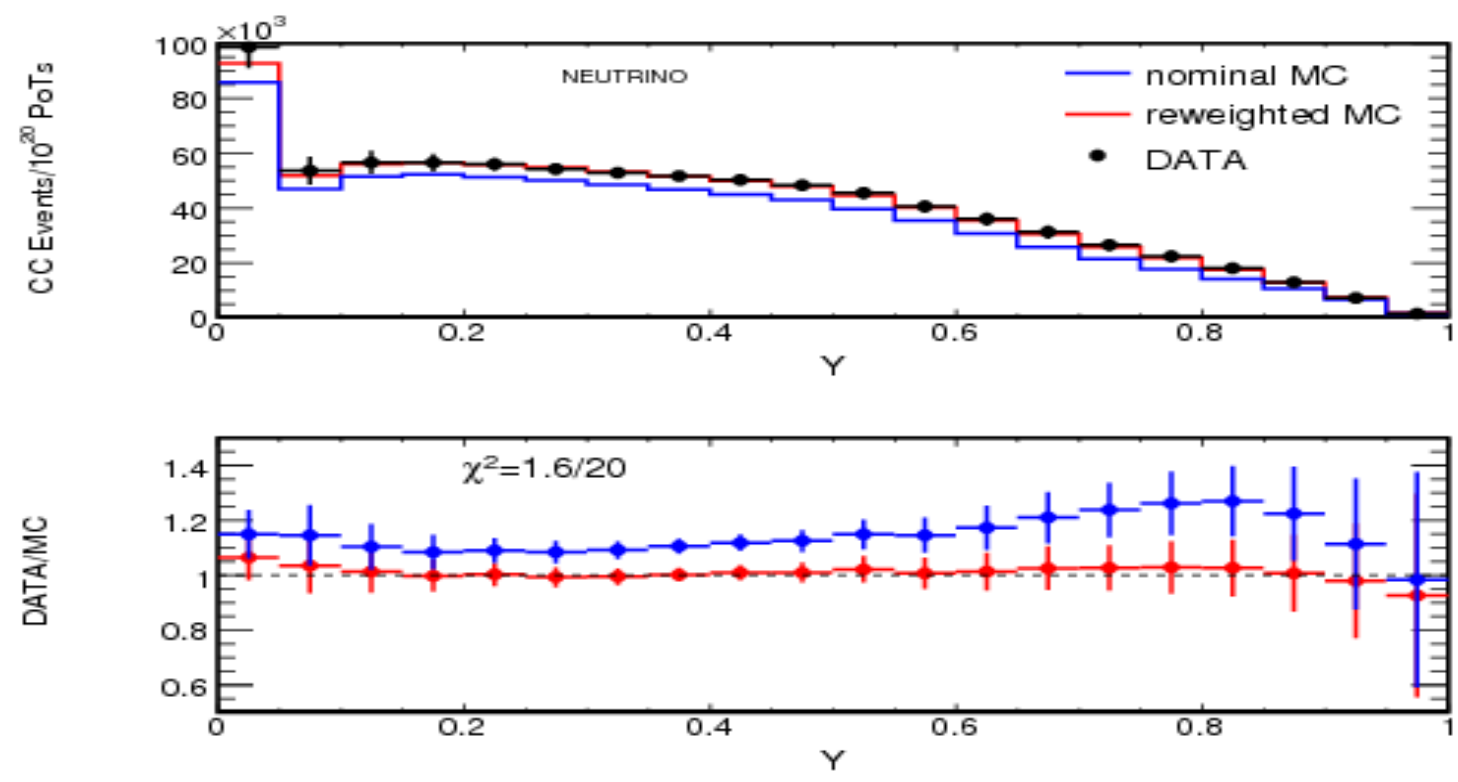

(a)
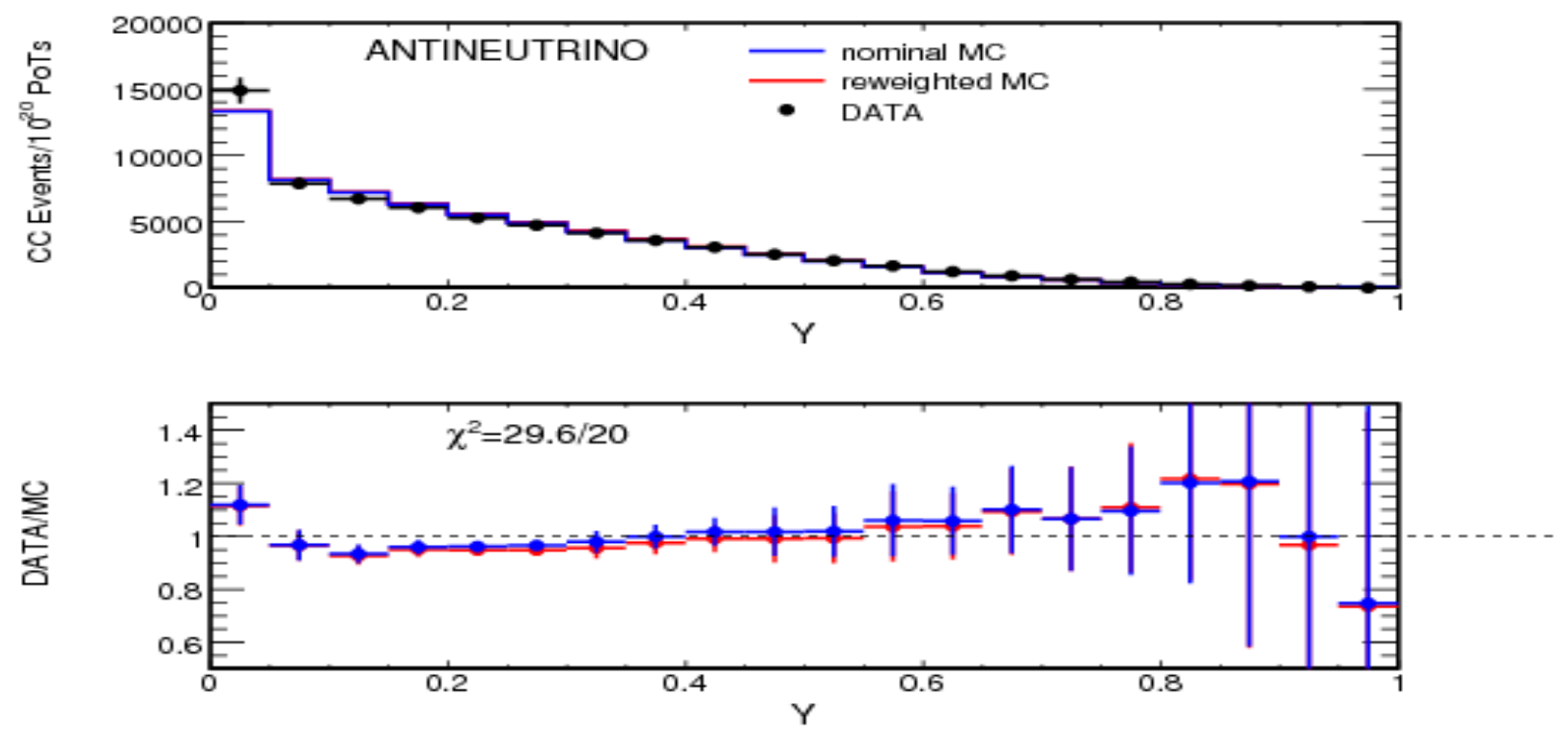

(b)

Figure 6.19: Comparison of data and MC simulation distribution of inelasticity for neutrinos(a) and antineutrinos(b) in the forward field mode. The points show data, the blue curve shows nominal MC model and the red curve shows MC model after applying flux reweighting. The ratio of data to MC is shown below each distribution. The error bars show the statistical and systematic uncertainties added in quadrature. 
$\mathrm{y}$ and $\mathrm{z}$ track vertex distribution for neutrinos show a good agreement. These plots don't have the $2 \%$ offset in the data to $\mathrm{MC}$ ratio as seen in Fig. 6.16(a). The data to MC ratio for the $\mathrm{x}$ vertex distribution still has some asymmetry specially in $x<1.5 \mathrm{~m}$ region of the order of $2 \%$. The antineutrino distribution has larger error bars but shows an overall good agreement.

Fig. 6.22 shows the data and MC track end distribution for neutrinos and antineutrinos. The track end radial distribution for neutrinos(antineutrinos) looks similar to the distribution for antineutrinos(neutrinos) shown in Fig. 6.17. This is expected because in the reverse field polarity, $\mu^{-}$s are defocused and in the forward field polarity $\mu^{+}$s are defocused. The neutrino end vertices show a much better agreement between data and MC because the coil hole mis-modeling doesn't affect this sample, although the disagreement at the edge of the detector is still present. The antineutrino track end radial distribution shows disagreement of the order of $20 \%$ around the coil hole.

Fig. 6.23 shows the data and MC distribution for the kinematic variables $E_{\mu}, E_{s h w}$ and $\theta_{\mu}$. The agreement between data and MC improves significantly after reweighting the nominal flux. The level of agreement between data and MC looks similar to the forward field data. 


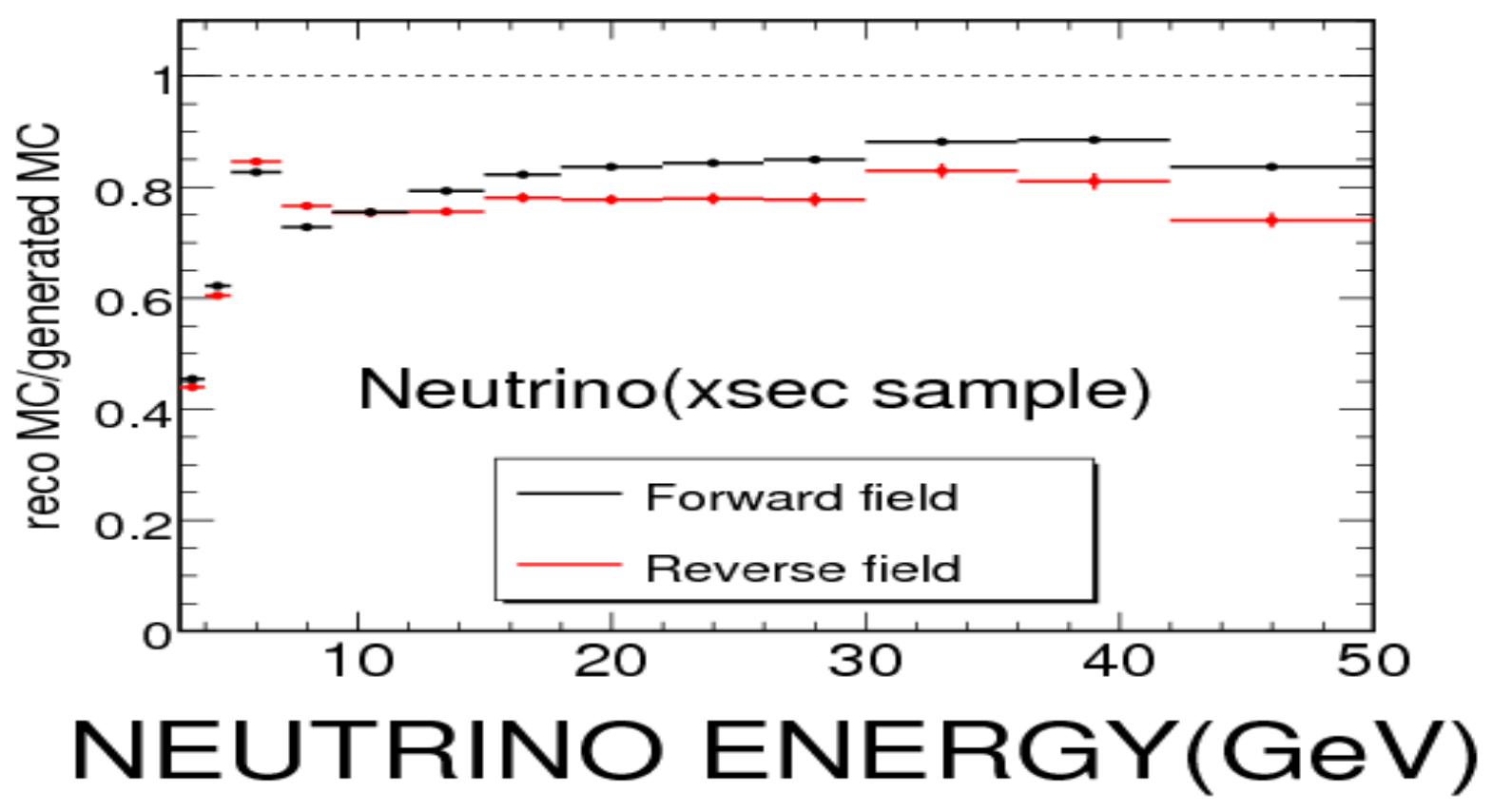

(a)

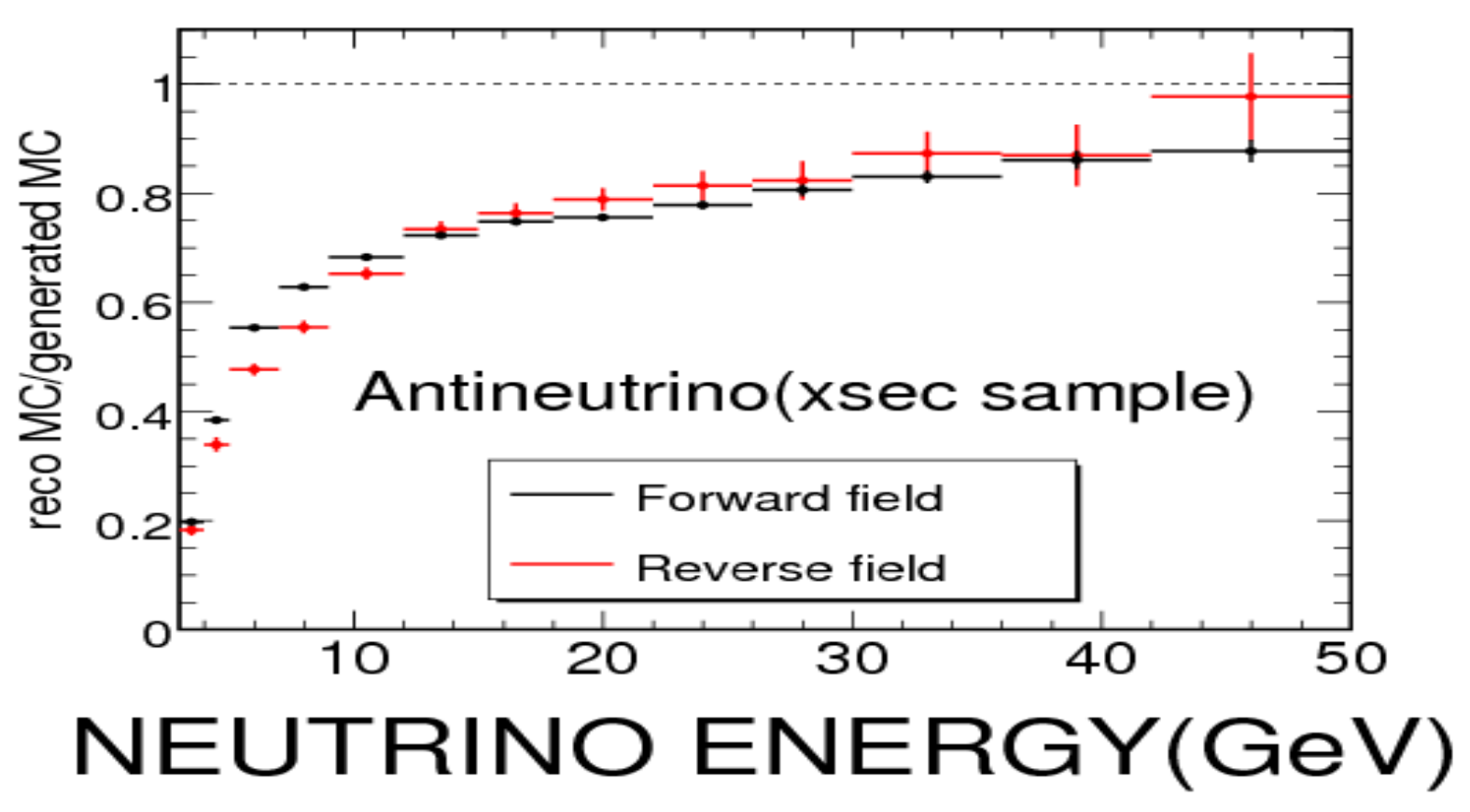

(b)

Figure 6.20: Comparison of reciprocal of acceptance correction for the reversed field and forward field neutrino(a) and antineutrino(b) sample. 

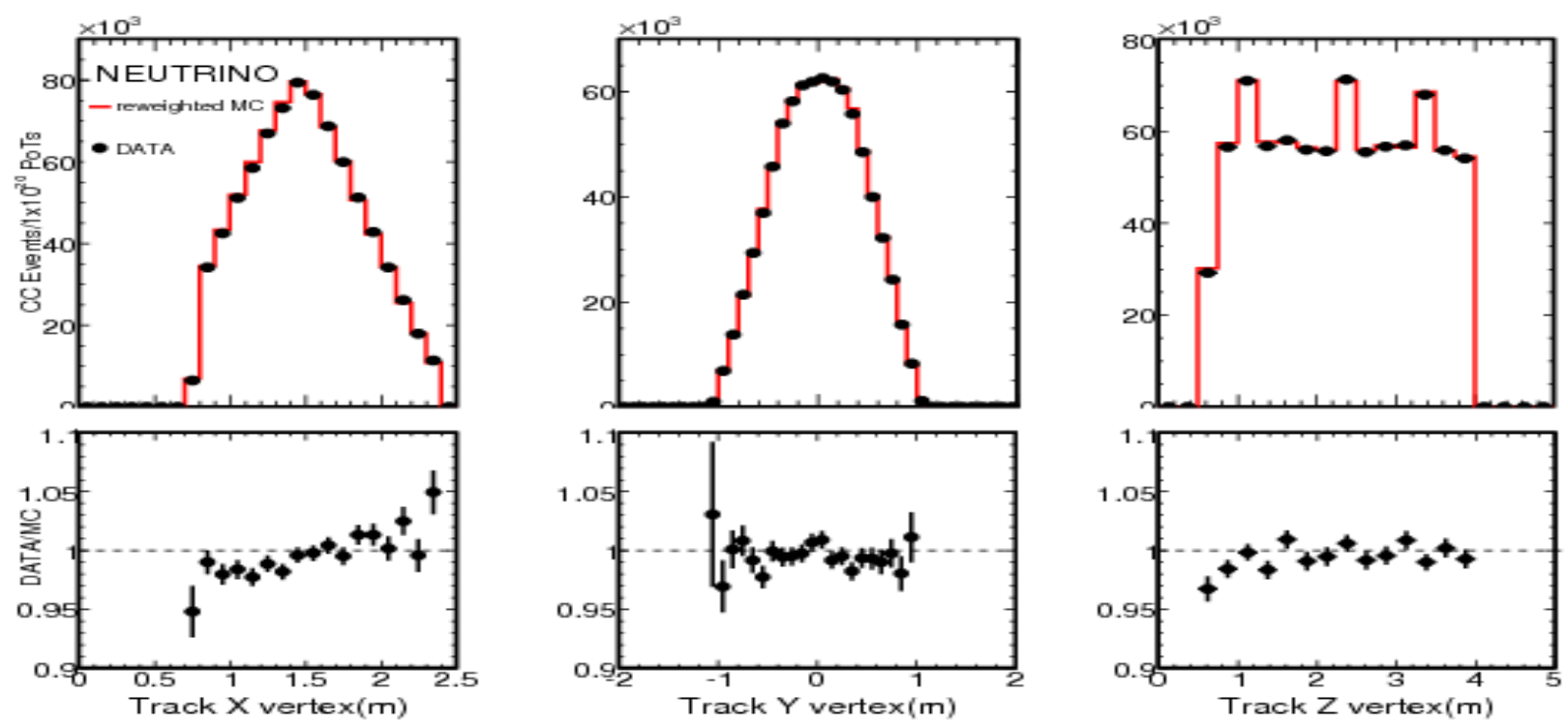

(a)
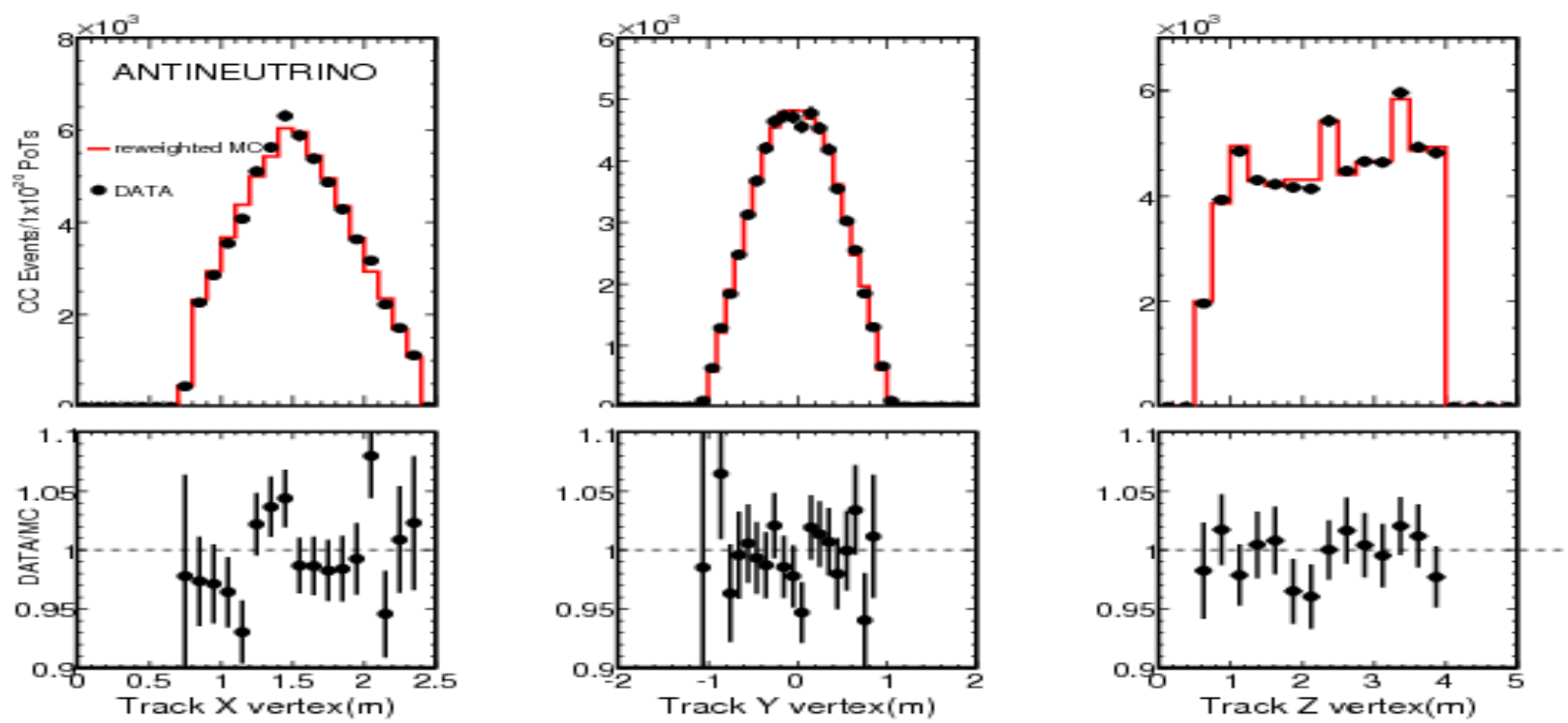

(b)

Figure 6.21: Comparison of data and MC simulation distribution for track beginning $\mathrm{x}, \mathrm{y}$ and $\mathrm{z}$ for neutrinos(a) and antineutrinos(b) in the reverse field mode. The points show data and red curve shows MC model after applying flux reweighting. The ratio of data to MC is shown below each distribution. The error bars show the statistical error only. 

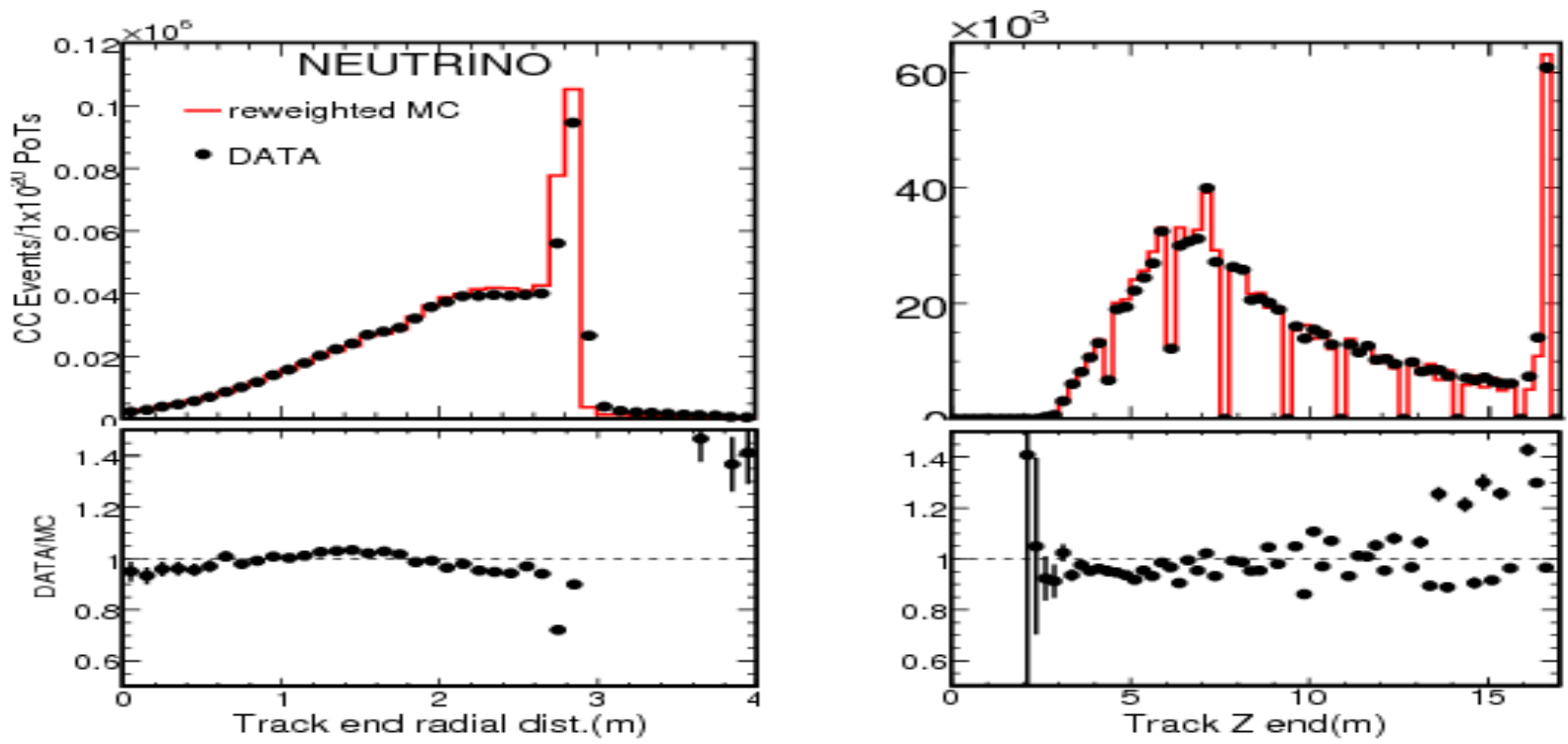

(a)
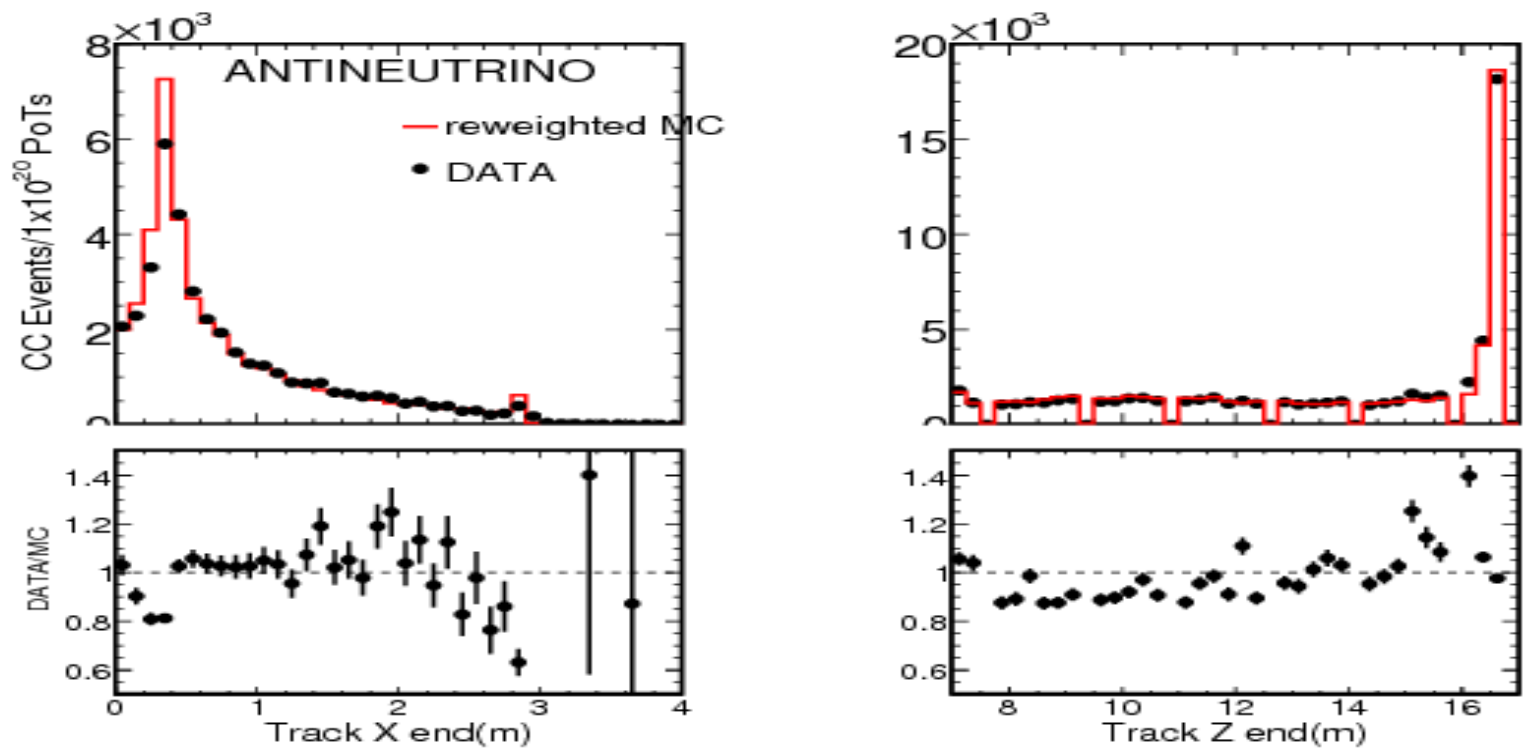

(b)

Figure 6.22: Comparison of data and MC simulation distribution for track end radial and track end $\mathrm{z}$ for neutrinos(a) and antineutrinos(b) in the reverse field mode. The points show data and red curve shows MC model after applying flux reweighting. The ratio of data to $\mathrm{MC}$ is shown below each distribution. The error bars show the statistical error only. 

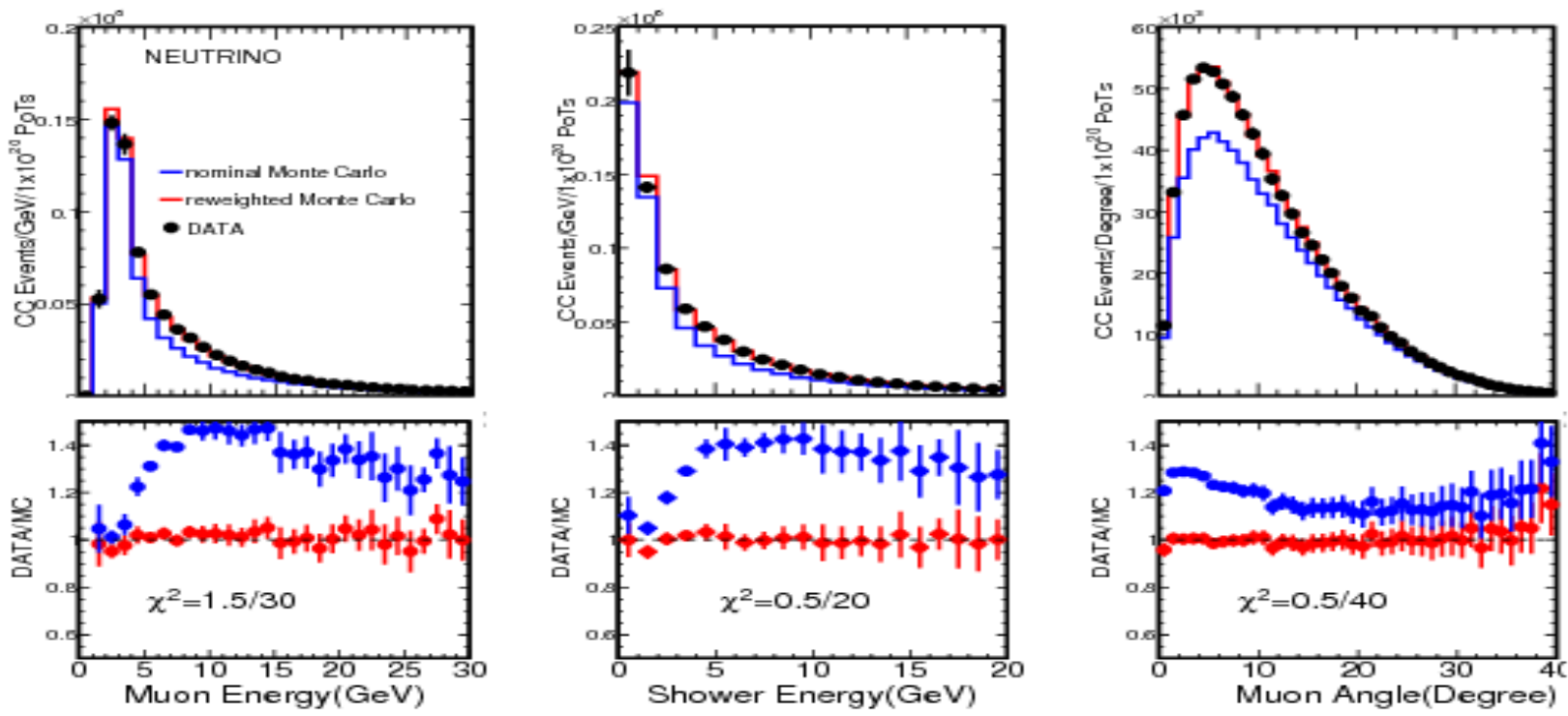

(a)
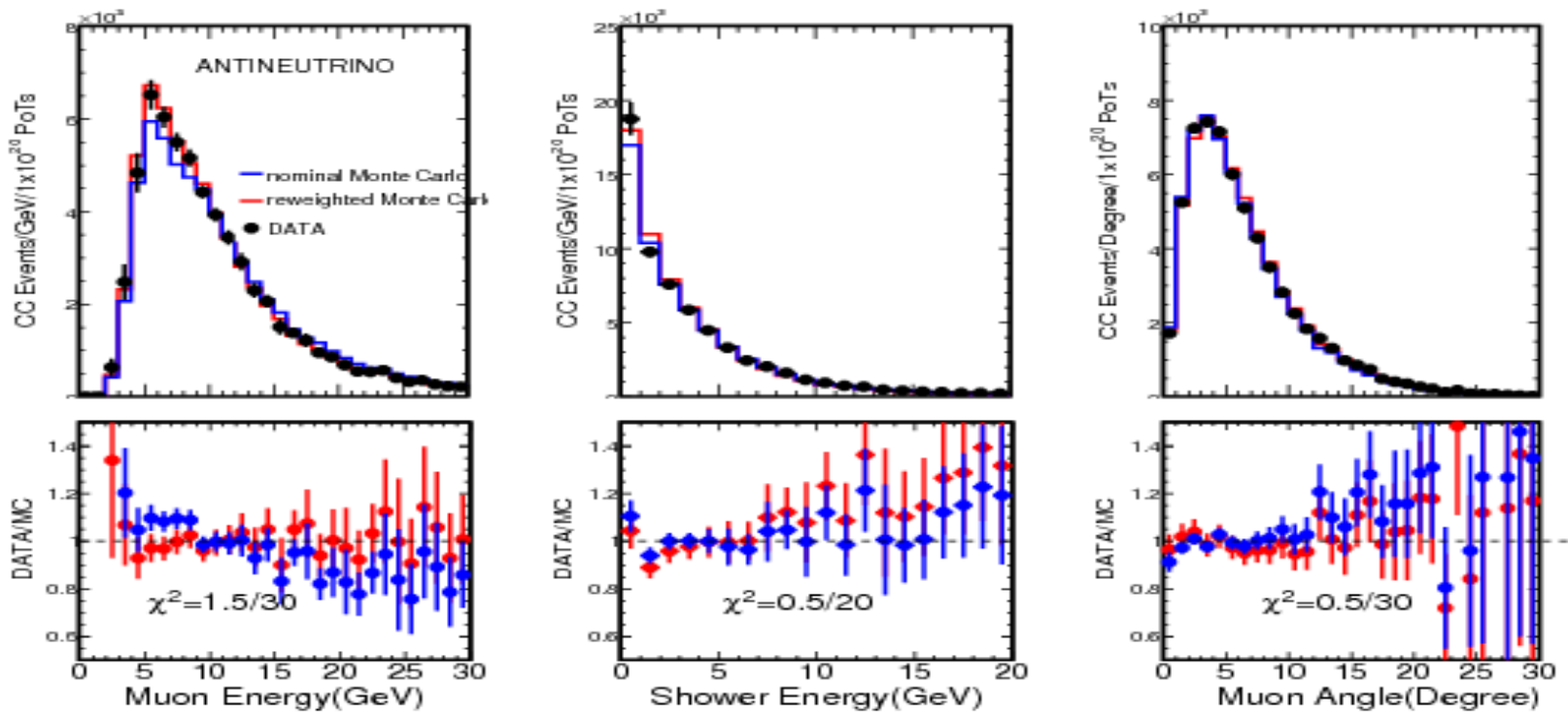

(b)

Figure 6.23: Comparison of data and MC simulation distribution for the kinematic variables $E_{\mu}$, $E_{s h w}$ and $\theta_{\mu}$ for neutrinos(a) and antineutrinos(b) in the reverse field mode. The points show data, the blue curve shows nominal MC model and the red curve shows MC model after applying flux reweighting. The ratio of data to $\mathrm{MC}$ is shown below each distribution. The error bars show the statistical and systematic uncertainties added in quadrature. 


\subsection{FLUX AND CROSS SECTION EXTRACTION}

The NuMI neutrino and antineutrino flux is simulated by using secondary hadron production data. The high intensity of the beam and the broad band energy spectrum makes it difficult to monitor the secondary particles which leads to large uncertainty in the simulation. We have used a modified version of the "low- $v$ " method[9] to extract flux. This method measures the flux using a subsample of the selected CC sample. The energy dependence of the CC cross section is measured by dividing the $\mathrm{CC}$ sample with the extracted flux. This chapter presents the details of the flux and cross section measurement and a description of the various systematic uncertainties affecting this analysis.

\subsection{OVERVIEW}

The CC cross section $\sigma_{C C}(E)$ depends on $N_{C C}^{v(\bar{v})}(E)$, the product of CC selected sample (section 6.1) and the acceptance correction (section 6.4) and $\Phi^{v(\bar{v})}(E)$, the flux in neutrino energy bins,

$$
\sigma_{C C}^{v(\bar{v})}(E) \propto \frac{N_{C C}^{v(\bar{v})}(E)}{\Phi^{v(\bar{v})}(E)} .
$$

The technique we use for extracting the flux is a variant of the low- $v$ method [9] used at higher energies by CCFR-96 [55] and NuTEV [20]. This flux extraction technique measures a relative flux, i.e. it gives the energy dependence of the flux and not the absolute normalization. It is conventional to plot the quantity $\sigma_{C C}(E) / E$ because at high energies(above $20 \mathrm{GeV}$ ) the DIS interaction is the dominant interaction and it rises linearly with energy (Eq. 1.33).

The MC simulation is used to calculate the acceptance correction and to determine the back- 
ground from NC and wrong sign events. The contamination in each sample is subtracted from the data. The neutrinos are binned into 13 bins and antineutrinos are binned into 11 bins. The size of the energy bins were selected such that the purity in any given energy bin ( defined as the ratio of the number of reconstructed events having their true energy between the energy limits of that bin to the total number of reconstructed events in that bin) is around 50\%. Fig. 7.1 shows the plot of purity versus neutrino energy.

Once the shape of cross section with energy is measured, it is normalized to the world cross section value above $30 \mathrm{GeV}\left(0.675 \pm 0.009 \times 10^{-38} \mathrm{~cm}^{2} / \mathrm{GeV}\right)[3]$. Fig. 7.2 shows the steps involved in the analysis in the form of a flow chart. To remove any effects of the input flux on the result, the analysis is re-iterated by reweighting the input flux with the ratio of $\Phi_{N E W}(E) / \Phi_{0}(E)$, where $\Phi_{N E W}(E)$ is the extracted flux and $\Phi_{0}(E)$ is the default MC flux.

\subsection{FLUX EXTRACTION (LOW $v$ METHOD)}

Eq. 1.33 for DIS charged current interaction can be written in terms of $\frac{d^{2} \sigma}{d x d v}$, using the substitution $y E=v$,

$$
\frac{d^{2} \sigma^{v(\bar{v})}}{d x d v}=\frac{G_{F}^{2} M}{\pi}\left[\left(1-\frac{v}{E}-\frac{M x v}{2 E^{2}}+\frac{v^{2}}{2 E^{2}} \frac{1+2 M x / v}{1+R_{L}}\right) F_{2} \pm \frac{v}{E}\left(1-\frac{v}{2 E}\right) x F_{3}\right]
$$

Integrating over $x$ gives

$$
\frac{d \sigma^{v(\bar{v})}}{d v}=\frac{G_{F}^{2} M}{\pi}\left(\int F_{2} d x\right)\left[1-\frac{v}{E}\left(1 \mp \frac{\int x F_{3}}{\int F_{2}}\right)+\frac{v^{2}}{2 E^{2}}\left(1 \mp \frac{\int x F_{3}}{\int F_{2}}+\frac{\int F_{2} R_{T E R M}}{\int F_{2}}\right)\right],
$$

where,

$$
R_{T E R M}=\left(\frac{1+2 M x / v}{1+R_{L}(x)}-\frac{M x}{v}-1\right) .
$$

Multiplying both sides of Eq. 7.3 with $\Phi(E)$ gives

$$
\frac{d N}{d v}=\Phi(E) A\left(1+\frac{B}{A} \frac{v}{E}-\frac{C}{A} \frac{v}{2 E^{2}}\right)
$$

where, 

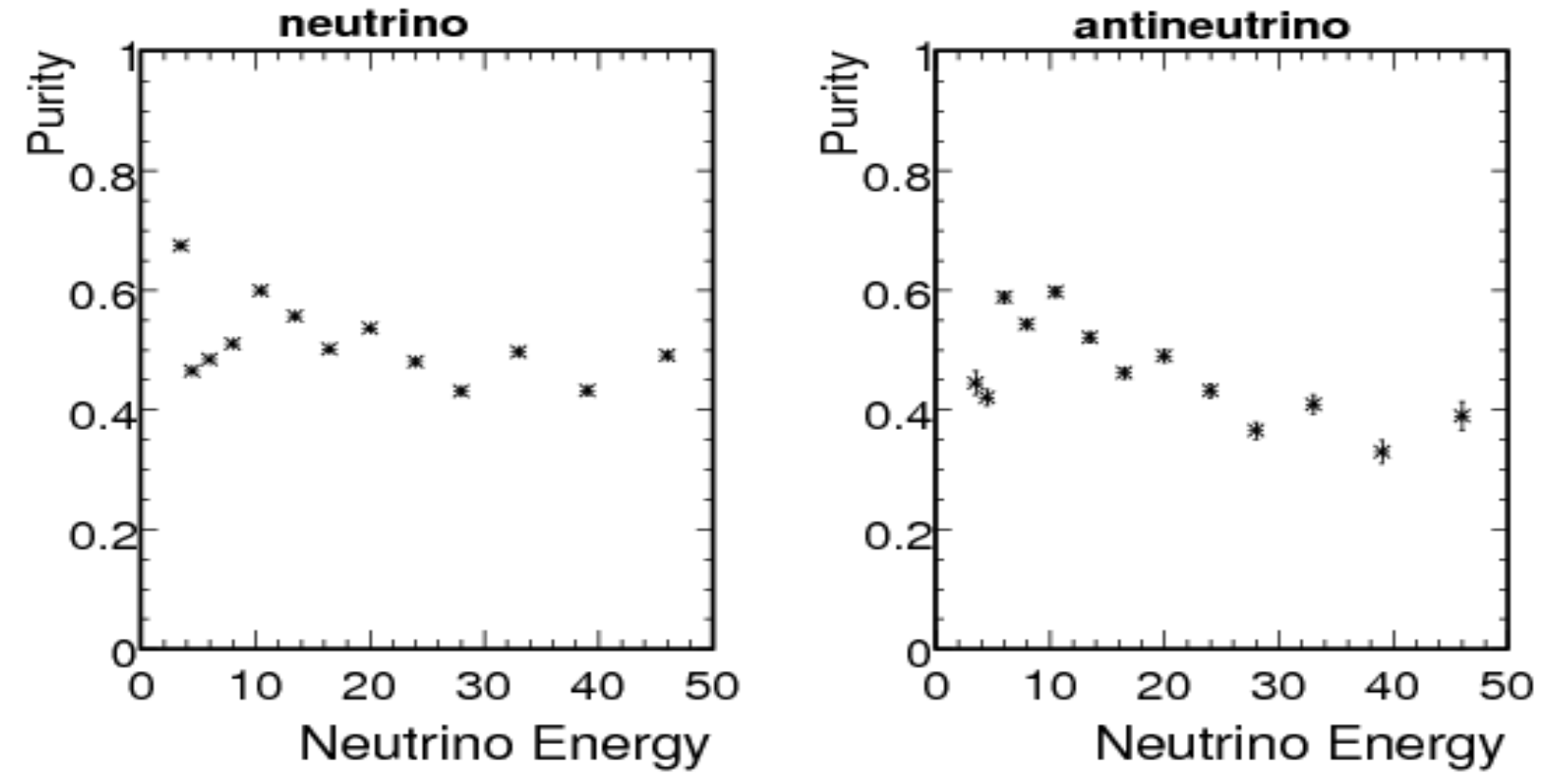

Figure 7.1: Purity as a function of energy for neutrino(left) and antineutrino(right) CC selected sample. 


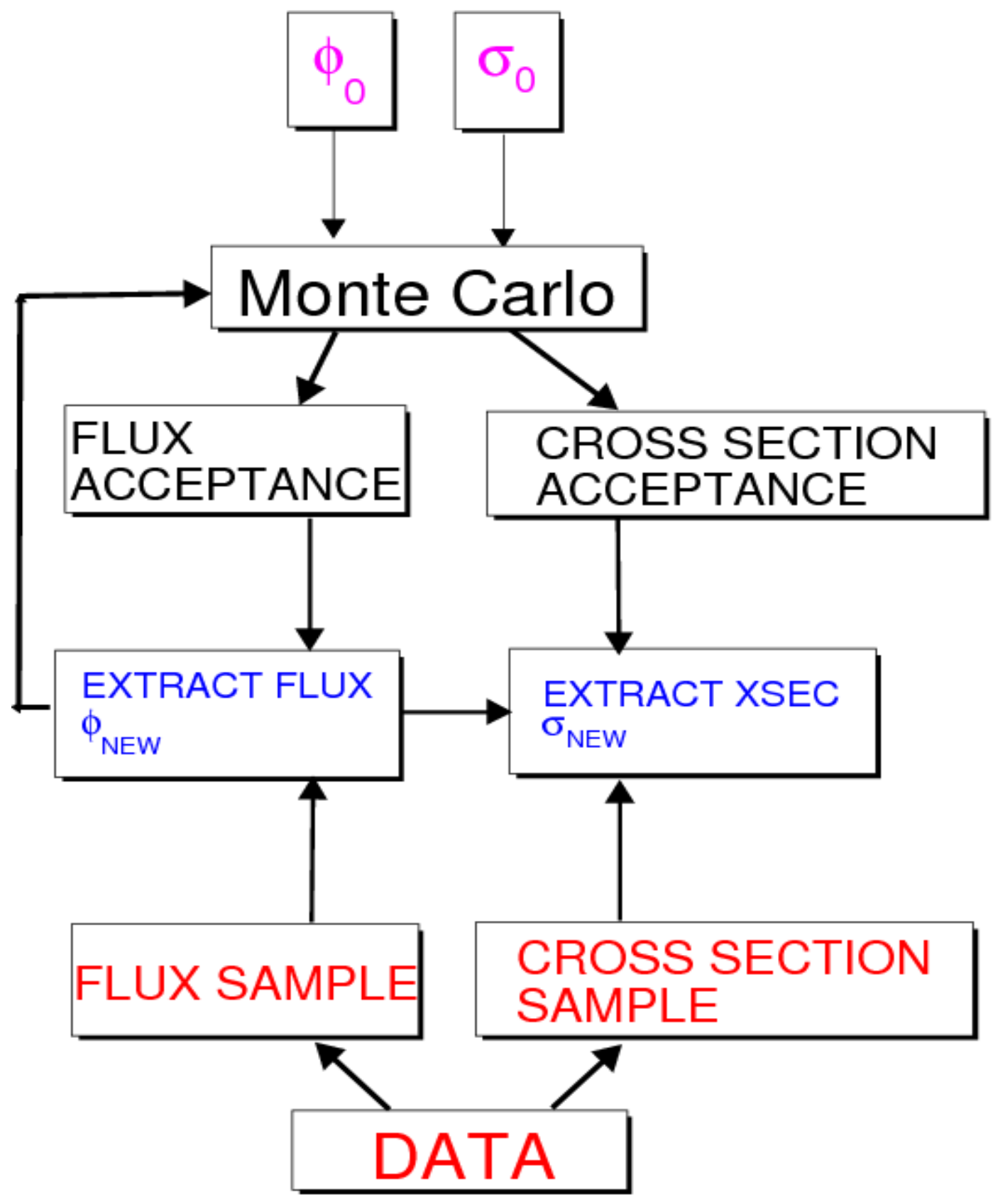

Figure 7.2: Flow diagram for extracting the flux and cross section. $\Phi_{0}$ and $\sigma_{0}$ are the predicted flux and cross section from the model, $\Phi_{N E W}$ and $\sigma_{N E W}$ are the extracted flux and cross section. 


$$
\begin{aligned}
& A=\frac{G_{F}^{2} M}{\pi} \int F_{2}(x) d x \\
& B=-\frac{G_{F}^{2} M}{\pi} \int\left(F_{2}(x) \mp x F_{3}(x)\right) d x, \text { and } \\
& C=B-\frac{G_{F}^{2} M}{\pi} \int F_{2}(x) R_{T E R M} d x
\end{aligned}
$$

For QEL interactions $A, B$ and $C$ will be expressed in terms of form factors.

In the limit $v \rightarrow 0$, terms proportional to $v$ vanish in Eq. 7.5 and the number of events is directly proportional to flux in a given energy bin

$$
\left.\Phi(E) \propto \frac{d N(E)}{d v}\right|_{\nu \rightarrow 0}
$$

where, the constant of proportionality $A$ is equal for neutrinos and antineutrinos. We apply a finite $v$ value which requires Eq. 7.7 to be corrected for the energy dependence from the terms $v / E$ and $(v / E)^{2}$.

$$
\Phi(E) \propto \frac{\int_{0}^{v_{0}} \frac{d N}{d v} d v}{\int_{0}^{v_{0}} d v\left[1+\frac{B}{A} \frac{v}{E}-\frac{C}{A} \frac{v^{2}}{2 E^{2}}\right]} .
$$

The cross section model calculates this correction term

$$
\operatorname{Corr}(E)=\frac{\sigma\left(v<v_{0}, E \rightarrow \infty\right)}{\sigma\left(v<v_{0}, E\right)},
$$

where, $\sigma\left(v<v_{0}, E\right)$ is the integrated cross section below the $v \operatorname{cut}\left(v_{0}\right)$ at energy $\mathrm{E}$ and $\sigma(v<$ $\left.v_{0}, E \rightarrow \infty\right)$ is its value in the high energy limit. Fig. 7.3 shows the size of the correction for neutrino and antineutrino samples. The flux given by

$$
\Phi(E) \propto \operatorname{Cor} r(E) \times \int_{0}^{v_{0}} \frac{d N}{d v} d v .
$$

is calculated by integrating the number of events up to a fixed $v$ and applying the correction $\operatorname{Corr}(E)$.

This correction introduces a model dependence in the flux determination. We account for uncertainty that this introduces in the flux by varying the model parameters and re-calculating 
the flux. The change in the correction when the model is varied is small $(\lesssim 1 \%)$ because it is a fractional term and the numerator and denominator are similarly affected.

To improve statistical precision on the flux measurement, we increase $v_{0}$ with energy while keeping the ratio $\frac{v}{E}$ small. $v_{o}$ is $1 \mathrm{GeV}$ for the $E_{v}<9 \mathrm{GeV}, 2 \mathrm{GeV}$ for $9<E_{v}<18$ and $5 \mathrm{GeV}$ for $E_{v}>18 \mathrm{GeV}$. Each of these samples are normalized separately to the world cross section at high energy. The details of the normalization procedure are given in section 7.4. From Fig. 7.3, the correction for neutrino is about $3 \%$ at $3 \mathrm{GeV}$ and for antineutrino is about $20 \%$ at $5 \mathrm{GeV}$. The stronger inelasticity dependence of the antineutrino cross section results in the much larger correction for antineutrinos.

\subsection{FLUX MEASUREMENT}

The flux sample is selected using the criteria discussed in section 6.1 with the addition of the maximum $v$ cut defined above. Fig. 7.4 shows the overlap of the flux and cross section samples for neutrino and antineutrino. The antineutrino sample peaks at low inelasticity. As a result there is a large overlap $(\sim 90 \%)$ of the cross section sample and the flux sample below $5 \mathrm{GeV}$. The overlap decreases with energy and is about $50 \%$ at $9 \mathrm{GeV}$ for the $v<1 \mathrm{GeV}$ flux sample. We therefore restrict our analysis for the antineutrinos to the region above $5 \mathrm{GeV}$. The raw number of events in the flux sample for neutrinos is 650950 and antineutrinos is 76058 . Table 7.1 shows the number of events passing the flux selection criteria in energy bins.

The MC sample is used to apply acceptance correction

$$
\operatorname{Acc}^{M C}\left(E, v<v_{0}\right)=\frac{N^{G E N}\left(E, v<v_{0}\right)}{N^{R E C O M C}\left(E, v<v_{0}\right)},
$$

where, $N^{G E N}\left(E, v<v_{0}\right)$ is the number of generated events within the fiducial volume in the energy bin $E$ with $v<v_{0}$ and $N^{R E C O M C}\left(E, v<v_{0}\right)$ is the number of smeared MC events within the fiducial volume in the energy bin $E$ with $v<v_{0}$. Fig. 7.5 shows the correction, which at high energies is close to one but at low energies is large because of the effect of the sample selection cuts. Acceptance correction has been discussed in section 6.4. Fig. 7.6 shows the fractional 

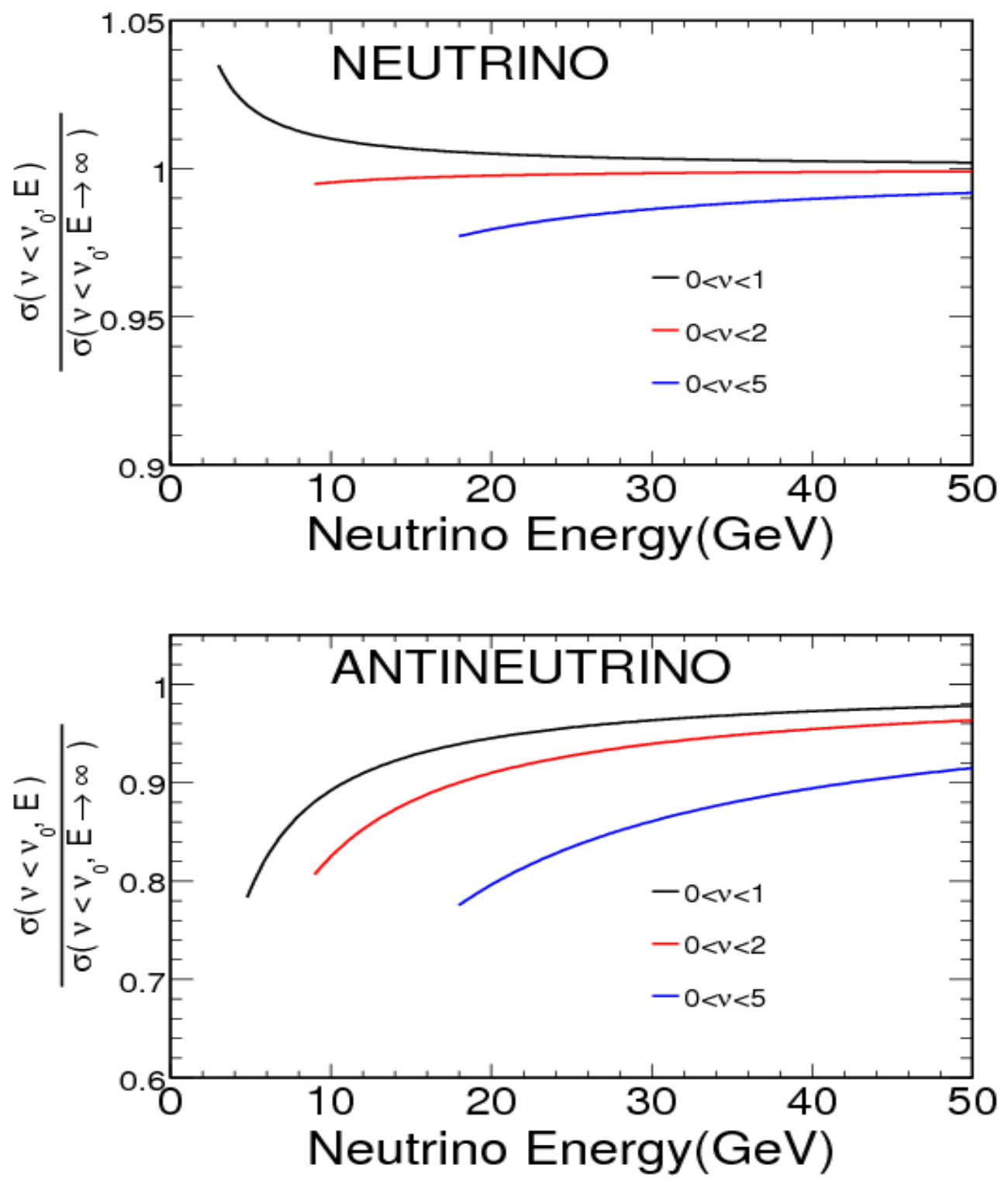

Figure 7.3: The correction applied to the flux sample for neutrino(a) and antineutrino(b). The black line shows the correction for $v<1 \mathrm{GeV}$ flux sample(3-50 GeV range for neutrinos, 5-50 $\mathrm{GeV}$ range for antineutrinos), red line shows the correction for $v<2 \mathrm{GeV}$ flux sample(9-50 GeV range) and blue line shows the correction for $v<5 \mathrm{GeV}$ flux sample(18-50 GeV range). 

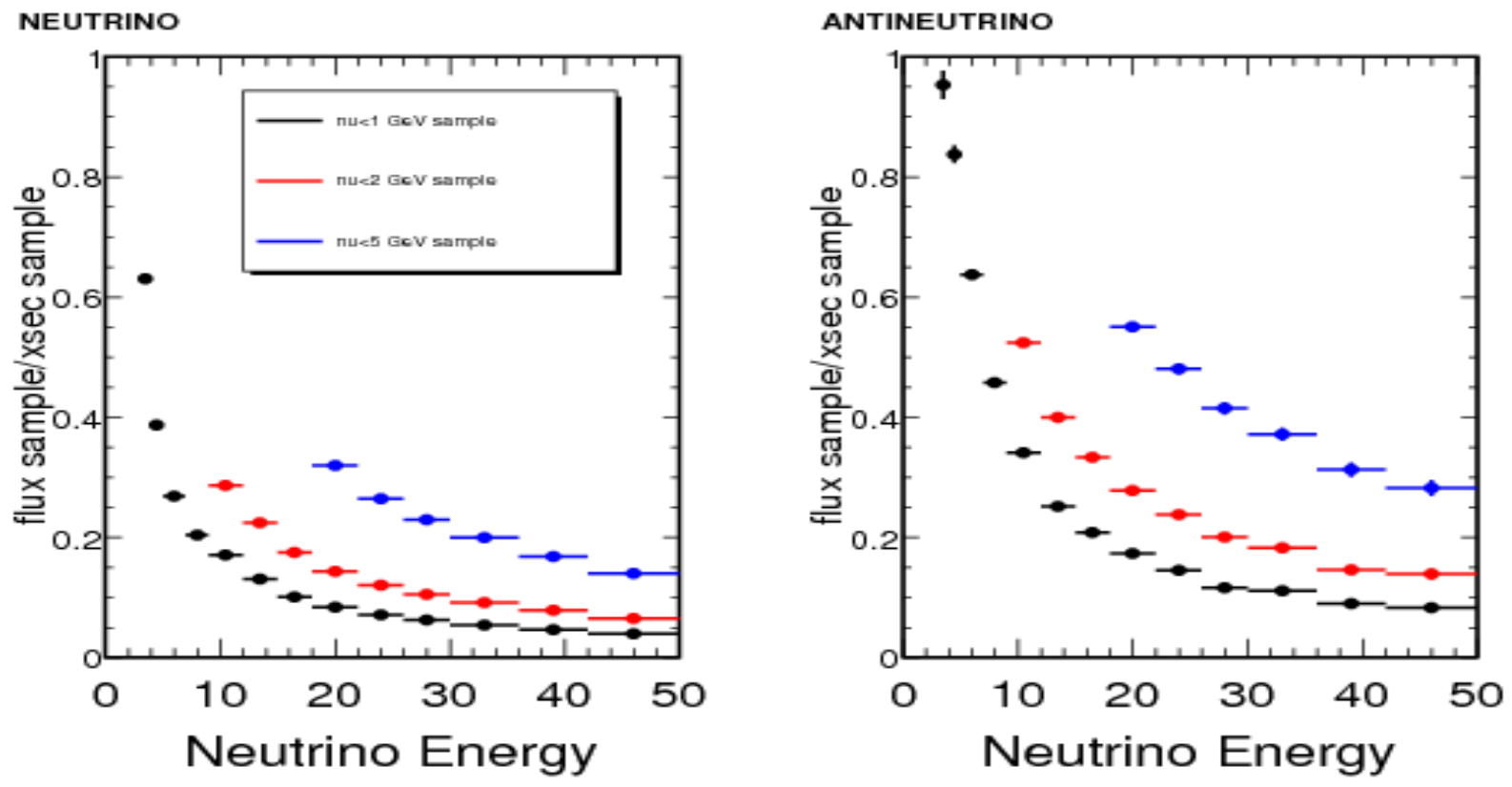

Figure 7.4: Overlap of flux and cross section sample for neutrino and antineutrino. The black curve shows the overlap of the $v<1 \mathrm{GeV}$ sample, red curve shows the overlap of the $v<2 \mathrm{GeV}$ sample and blue curve shows the overlap of the $v<5 \mathrm{GeV}$ sample. 


\begin{tabular}{|c|c|c|c|}
\hline Bin & Energy Range & Events in $v$ sample & Events in $\bar{v}$ sample \\
\hline \hline 1 & $3-4 \mathrm{GeV}$ & 239261 & - \\
\hline 2 & $4-5 \mathrm{GeV}$ & 94615 & - \\
\hline 3 & $5-7 \mathrm{GeV}$ & 66561 & 14137 \\
\hline 4 & $7-9 \mathrm{GeV}$ & 34252 & 12093 \\
\hline 5 & $9-12 \mathrm{GeV}$ & 60790 & 17672 \\
\hline 6 & $12-15 \mathrm{GeV}$ & 38474 & 9762 \\
\hline 7 & $15-18 \mathrm{GeV}$ & 22196 & 5532 \\
\hline 8 & $18-22 \mathrm{GeV}$ & 37286 & 7841 \\
\hline 9 & $22-26 \mathrm{GeV}$ & 20928 & 3972 \\
\hline 10 & $26-30 \mathrm{GeV}$ & 12544 & 2043 \\
\hline 11 & $30-36 \mathrm{GeV}$ & 11329 & 1608 \\
\hline 12 & $36-42 \mathrm{GeV}$ & 6910 & 831 \\
\hline 13 & $42-50 \mathrm{GeV}$ & 5804 & 567 \\
\hline
\end{tabular}

Table 7.1: Raw number of events in neutrino energy bins for the neutrino and antineutrino flux sample. 
contamination in the flux sample calculated from the MC which is subtracted bin by bin from the data.

Eq. 7.5 is derived for an isoscalar target. The MINOS iron detector is non-isoscalar with a $5.67 \%$ excess of neutrons over protons. A correction of about $-2 \%$ for neutrinos $(+2 \%$ for antineutrinos) is computed from the cross section model and used to convert our measurement into that expected for an isoscalar target. Fig. 7.7 shows the correction applied to neutrino and antineutrino flux sample.

Fig. 7.8 shows the ratio of extracted flux to simulated flux for neutrinos and antineutrinos. This ratio is large(40\% at $15 \mathrm{GeV}$ for neutrinos) and reflects the difficulty in simulating the beamline due to currently large uncertainties in particle production on a graphite target at NuMI energies. The ratio in Fig. 7.8 is applied as a weighting factor to the MC simulation to correct the input flux model. The systematic uncertainties on the extracted flux are discussed in detail in chapter 7.5. The measurement is dominated by systematic uncertainties at low energy and by statistical uncertainty at higher energies.

Table 7.2 and Fig. 7.11 show the extracted absolute flux for neutrino and antineutrino. The absolute flux is obtained by multiplying the ratio of data to MC flux with the simulated flux shown in Fig. 2.3. To obtain the true flux, radiative corrections are applied to the MC flux and the MC and data flux are separately normalized to the world cross section at high energy $\left(0.675 \pm 0.009 \times 10^{-38}\right.$ $\mathrm{cm}^{2} / \mathrm{GeV}$ ). The normalization constant for data and MC have a difference of about $3.5 \%$ out of which $2 \%$ is contributed by the radiative correction and the rest is contributed by the difference in data and $\mathrm{MC}$ weighted average in the high energy region. The default flux simulation calculates the flux at the center of the detector plane. This flux is smeared over a circular area of radius 0.75 $\mathrm{m}$, which is an approximation of our fiducial area shown in Fig. 7.9. The ratio of the smeared flux to the flux at the center of the detector is shown in Fig. 7.10.

The default MC flux has also been tuned using an alternative method described in [24]. Appendix C compares this flux tuning procedure with the low $v$ method.

The value of low $v$ that we choose to select our flux sample can have an effect on the analysis. The effect of modifying the low $v$ cut from $1 \mathrm{GeV}$ to $0.75 \mathrm{GeV}$ has been evaluated. The result of this study shown in Fig. 7.12 has effects of the order of $1 \%$ on the analysis which is within our statistical precision. 


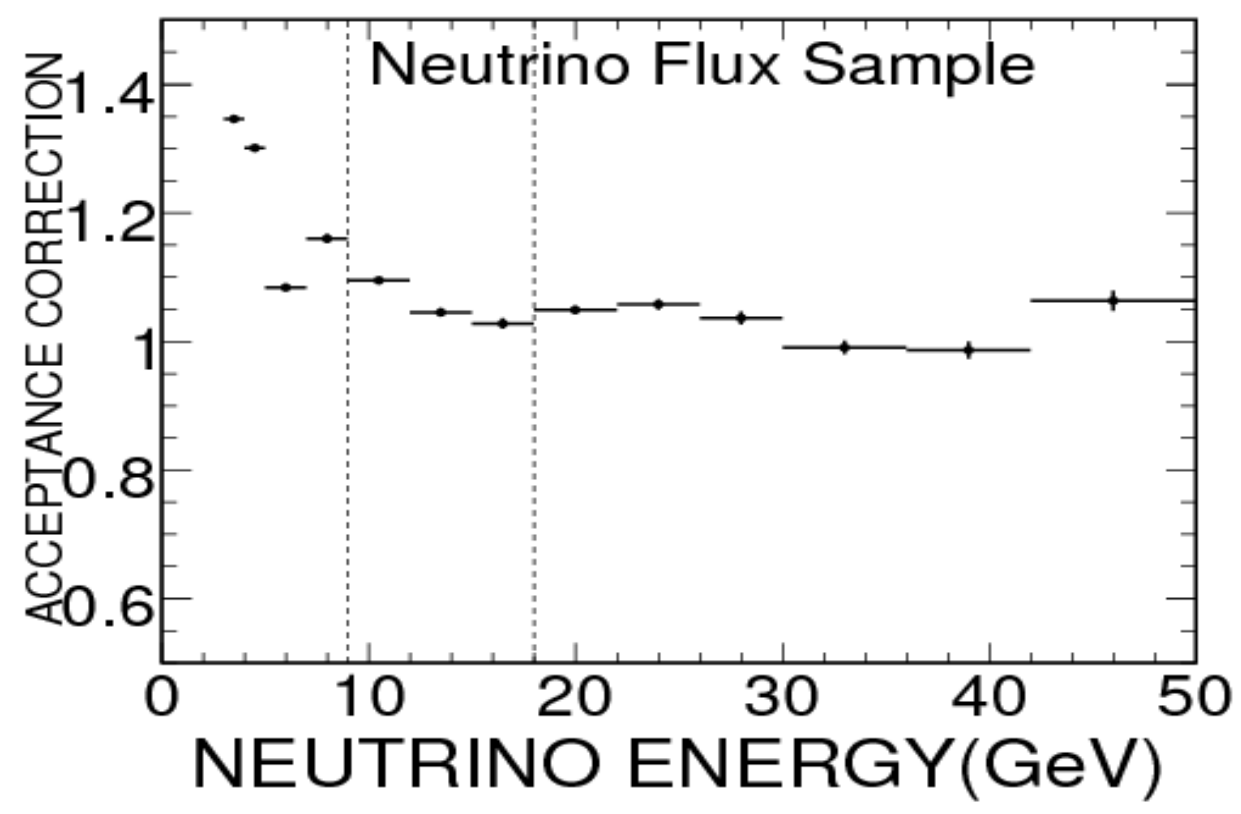

(a)

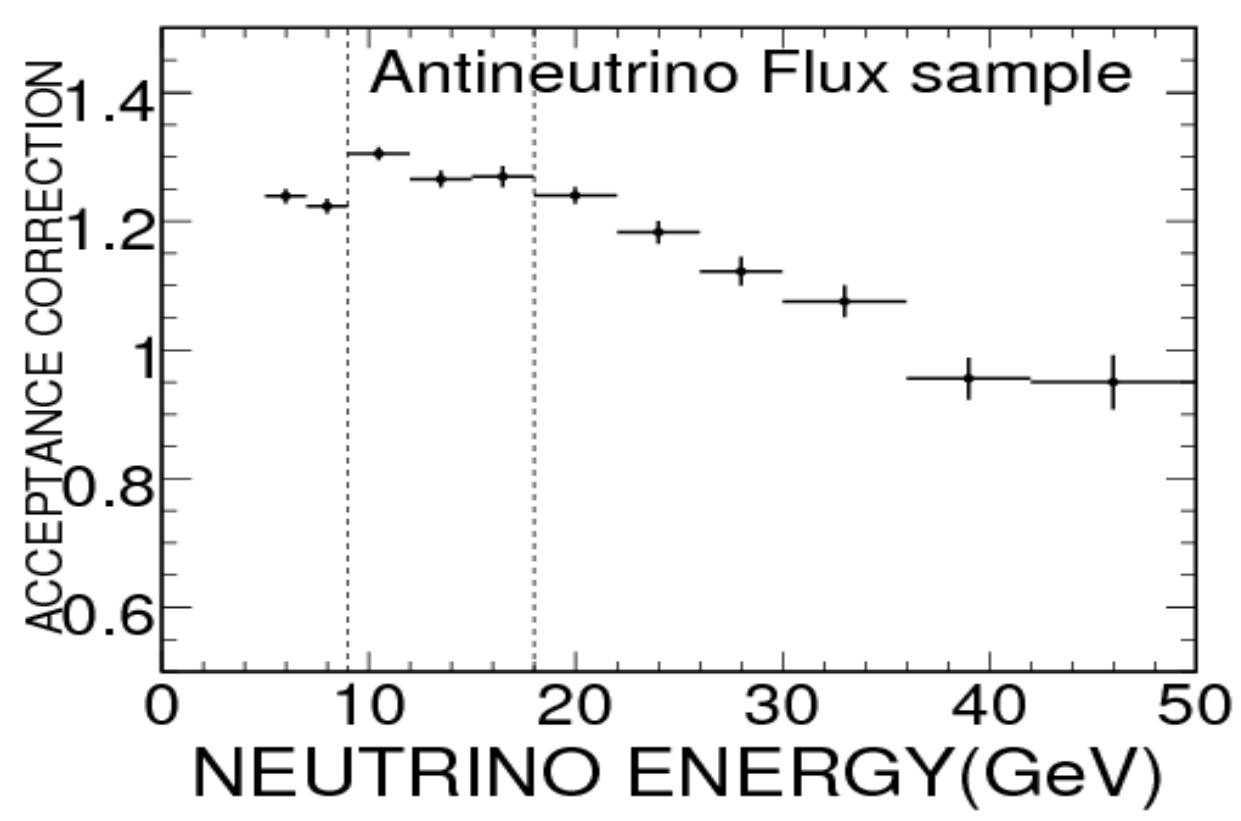

(b)

Figure 7.5: Acceptance correction from MC simulation for neutrino(a) and antineutrino(b) flux selected sample. The dashed lines show the energy values $(9 \mathrm{GeV}$ and $18 \mathrm{GeV})$ at which the value of the $v_{0}$ cut is increased. 


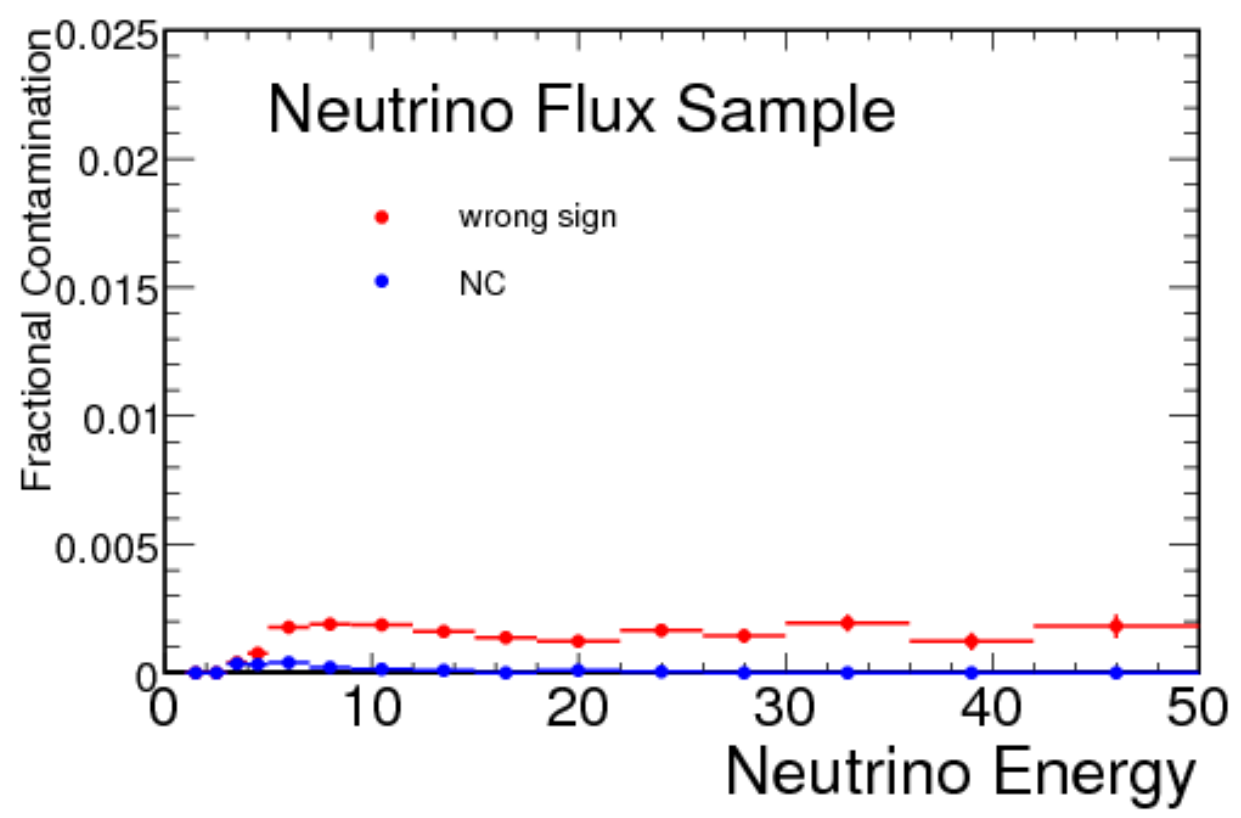

(a)

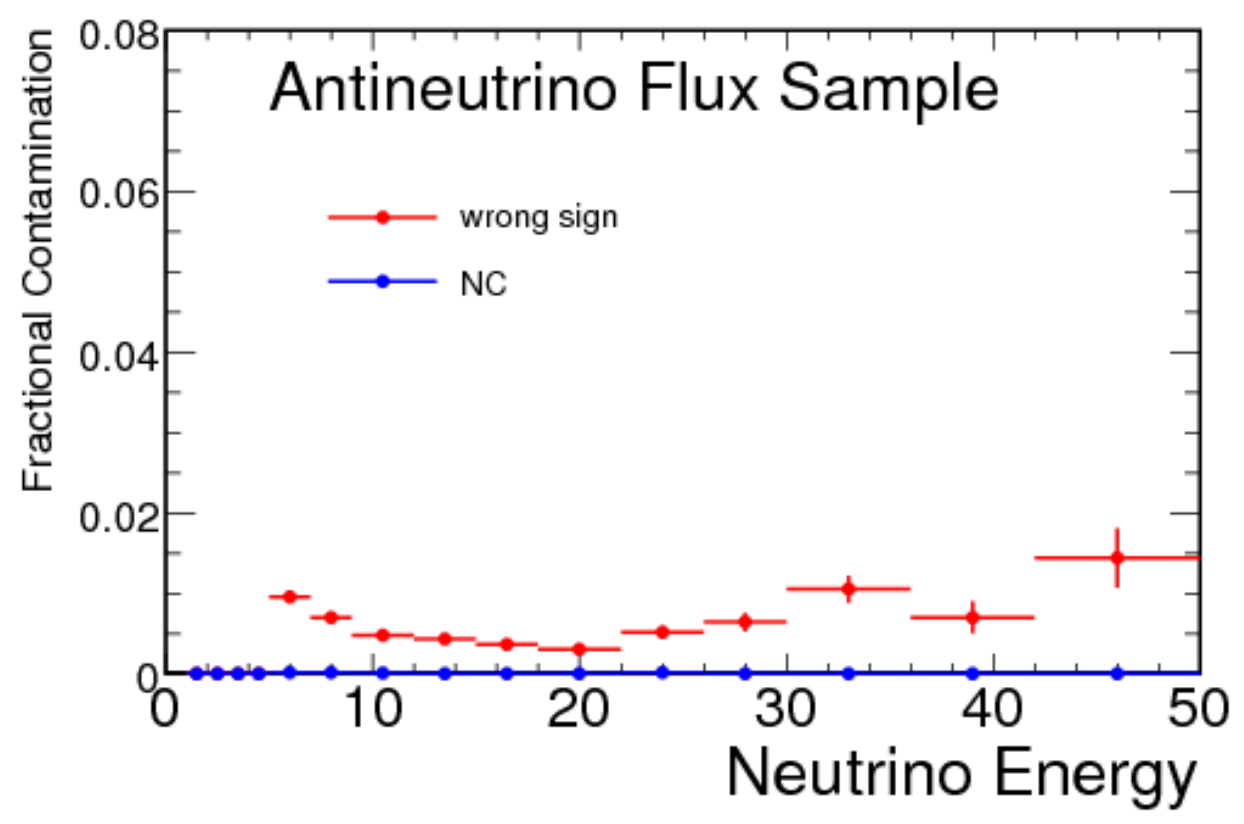

(b)

Figure 7.6: Fractional contamination calculated from the flux sample in the neutrino(a) and antineutrino(b) flux selected sample. The red curve shows the fractional wrong sign contamination and the blue curve shows the fractional NC contamination. 


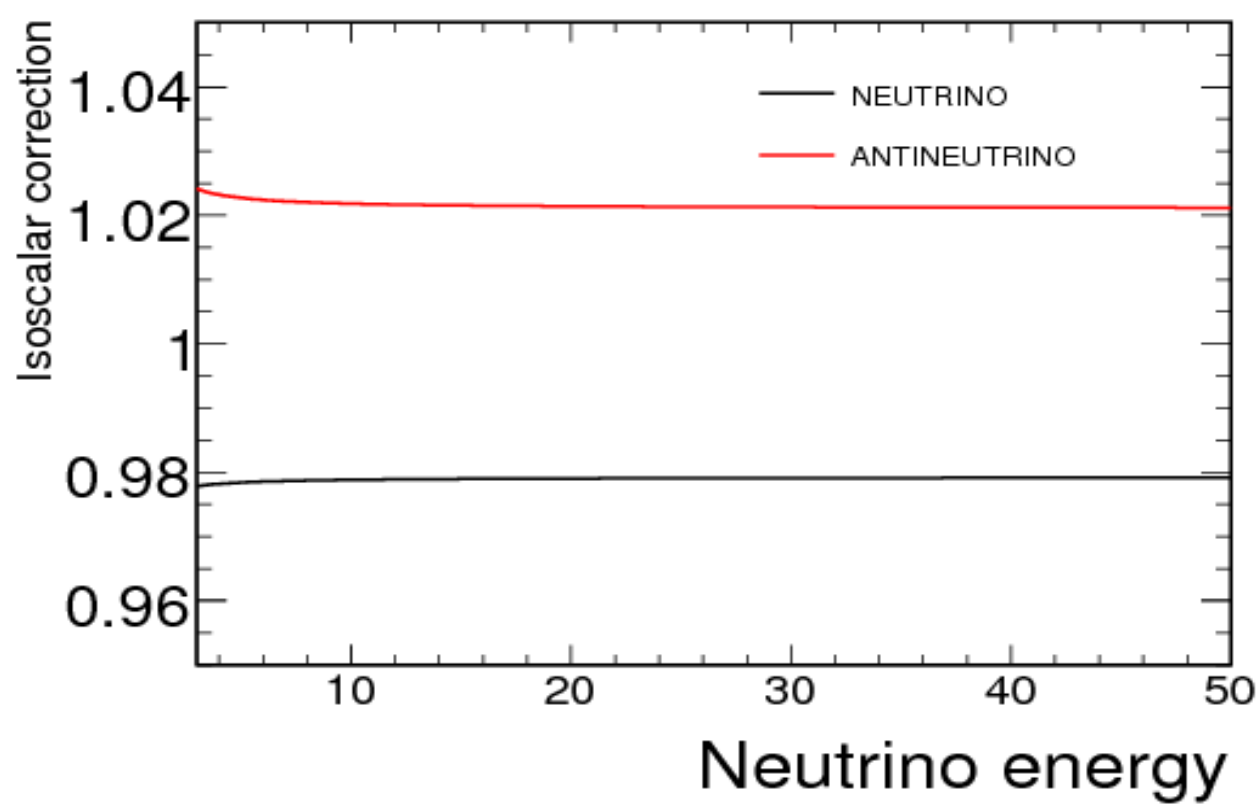

Figure 7.7: Isoscalar correction applied to the neutrino and antineutrino flux sample. The black line shows the correction for neutrino and the red line shows the correction for antineutrino. 


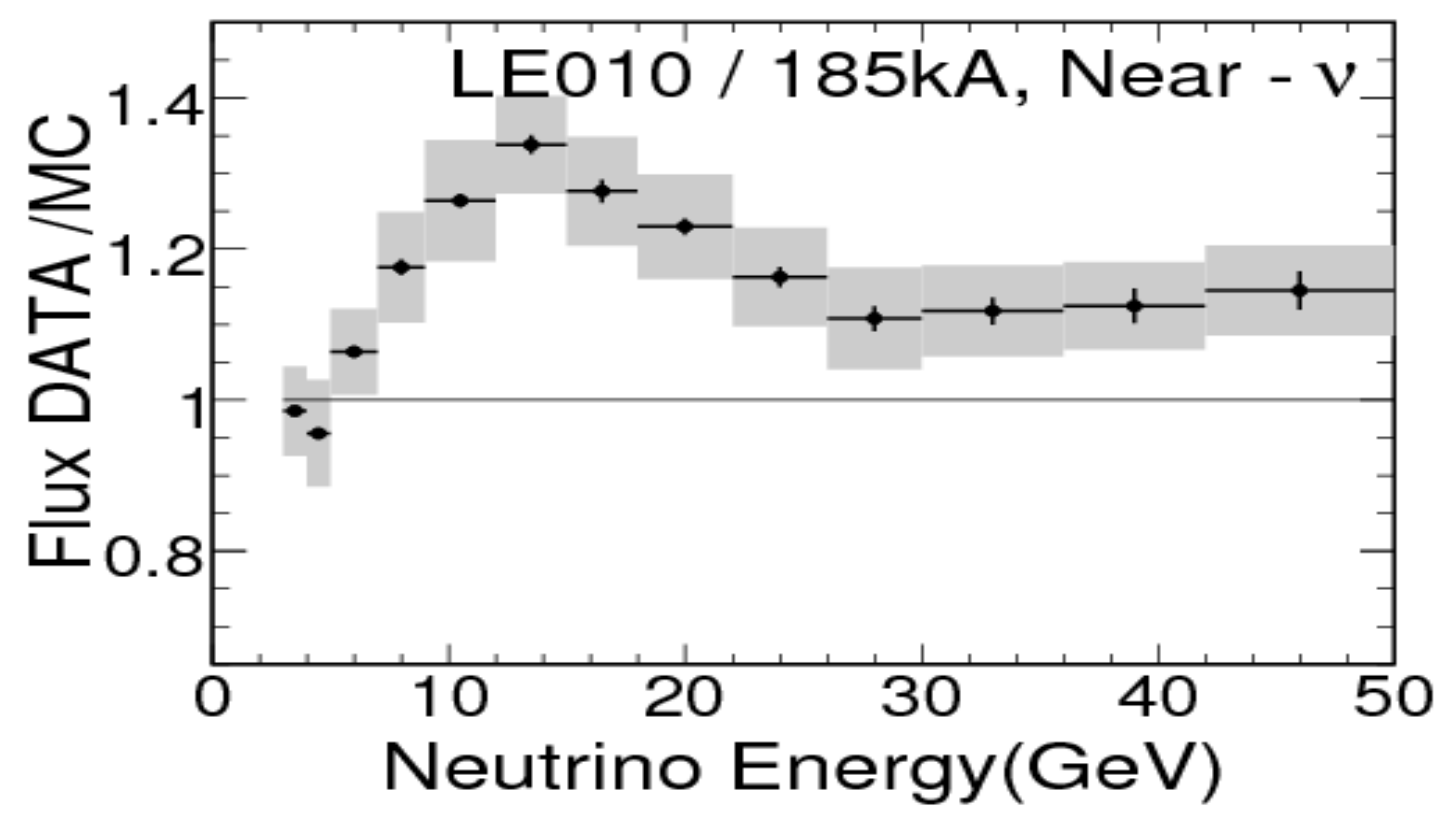

(a)

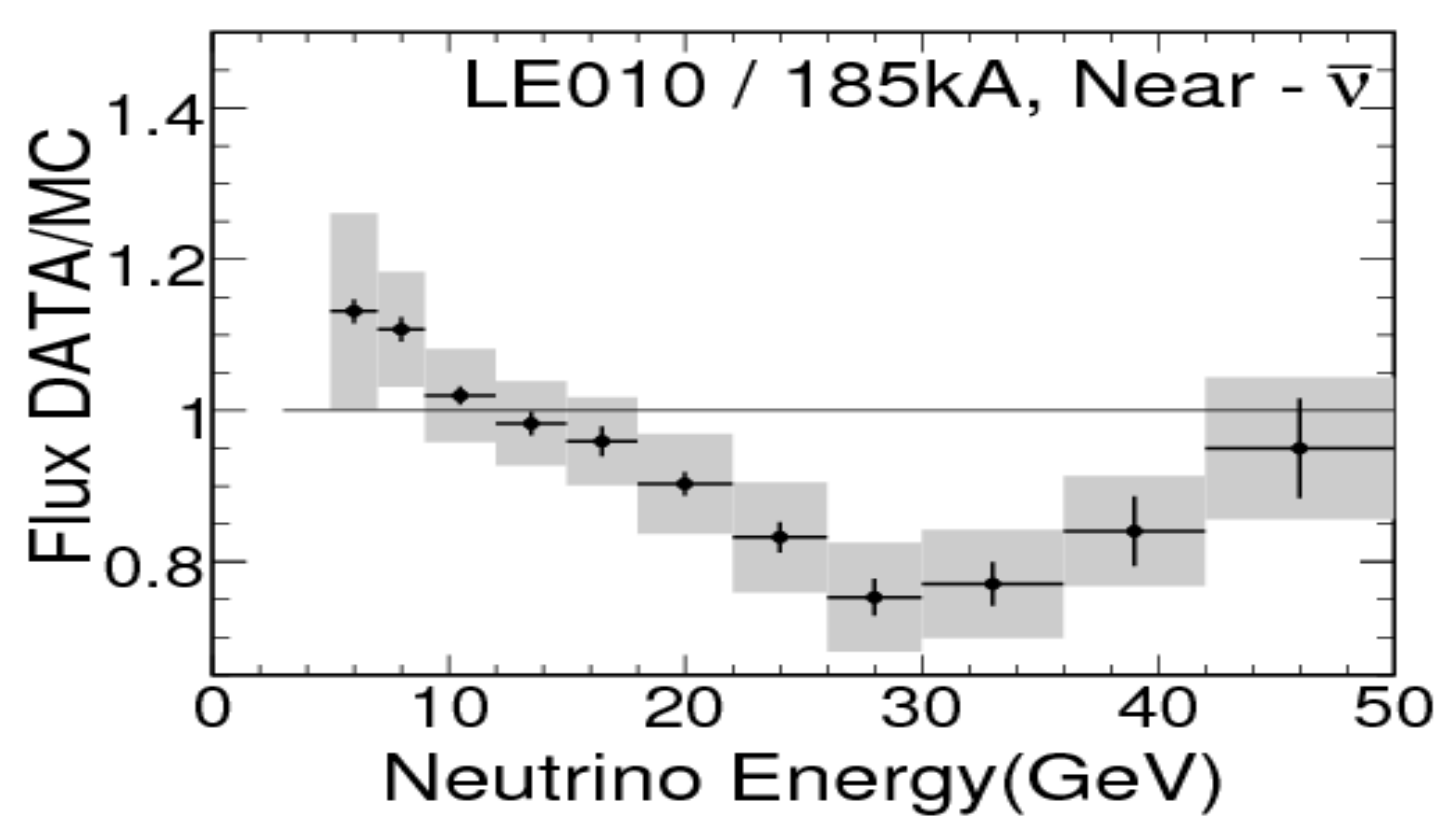

(b)

Figure 7.8: Ratio of the extracted flux to simulated flux for neutrinos(a) and antineutrinos(b) as a function of neutrino energy. The shaded box shows the statistical and systematic uncertainties added in quadrature and the error bars show the statistical error only. 


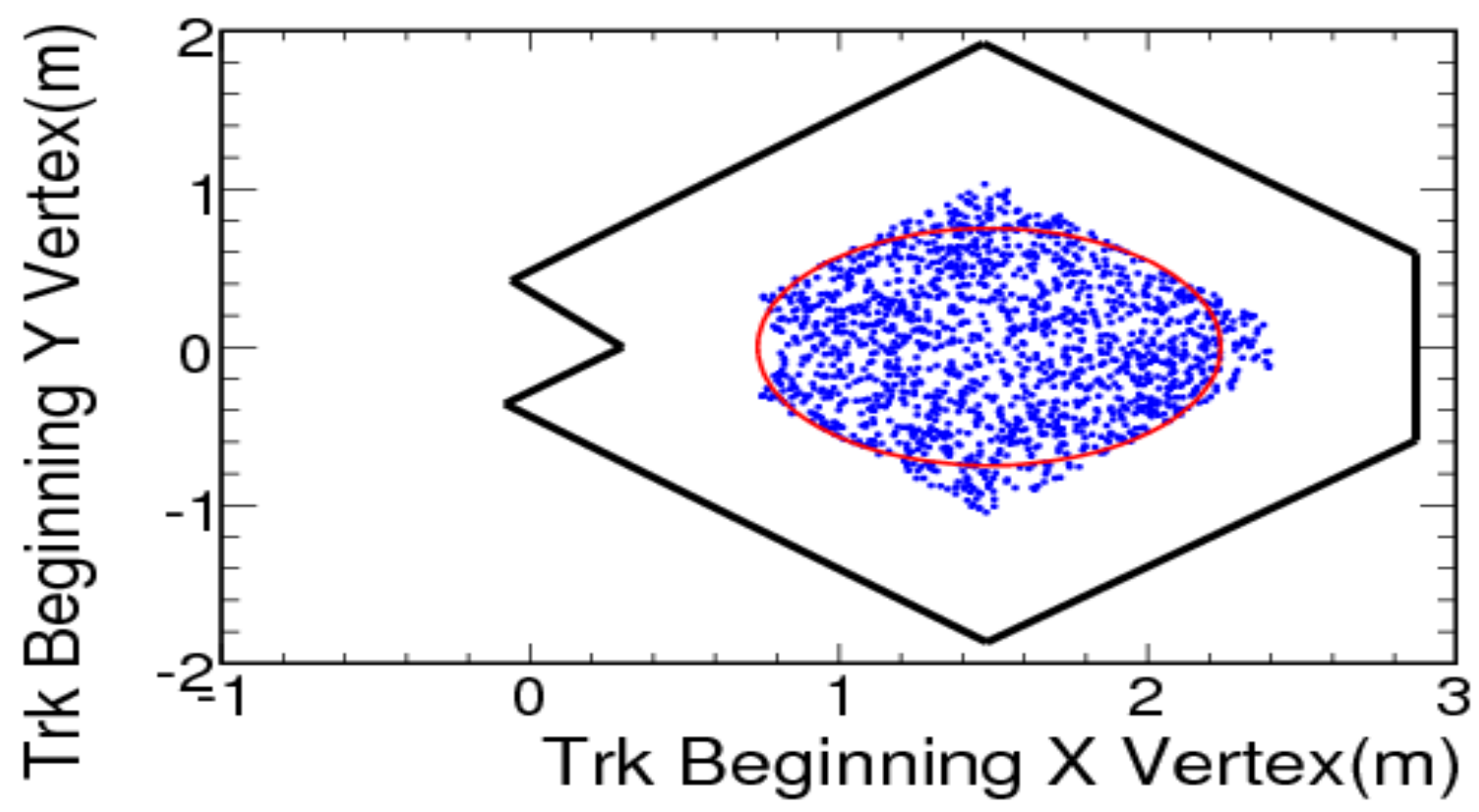

Figure 7.9: Comparison of the fiducial volume used in this analysis with a circular area of radius $0.75 \mathrm{~m}$. The circular area has been used to smear the MC flux that is calculated at the center of the detector. 


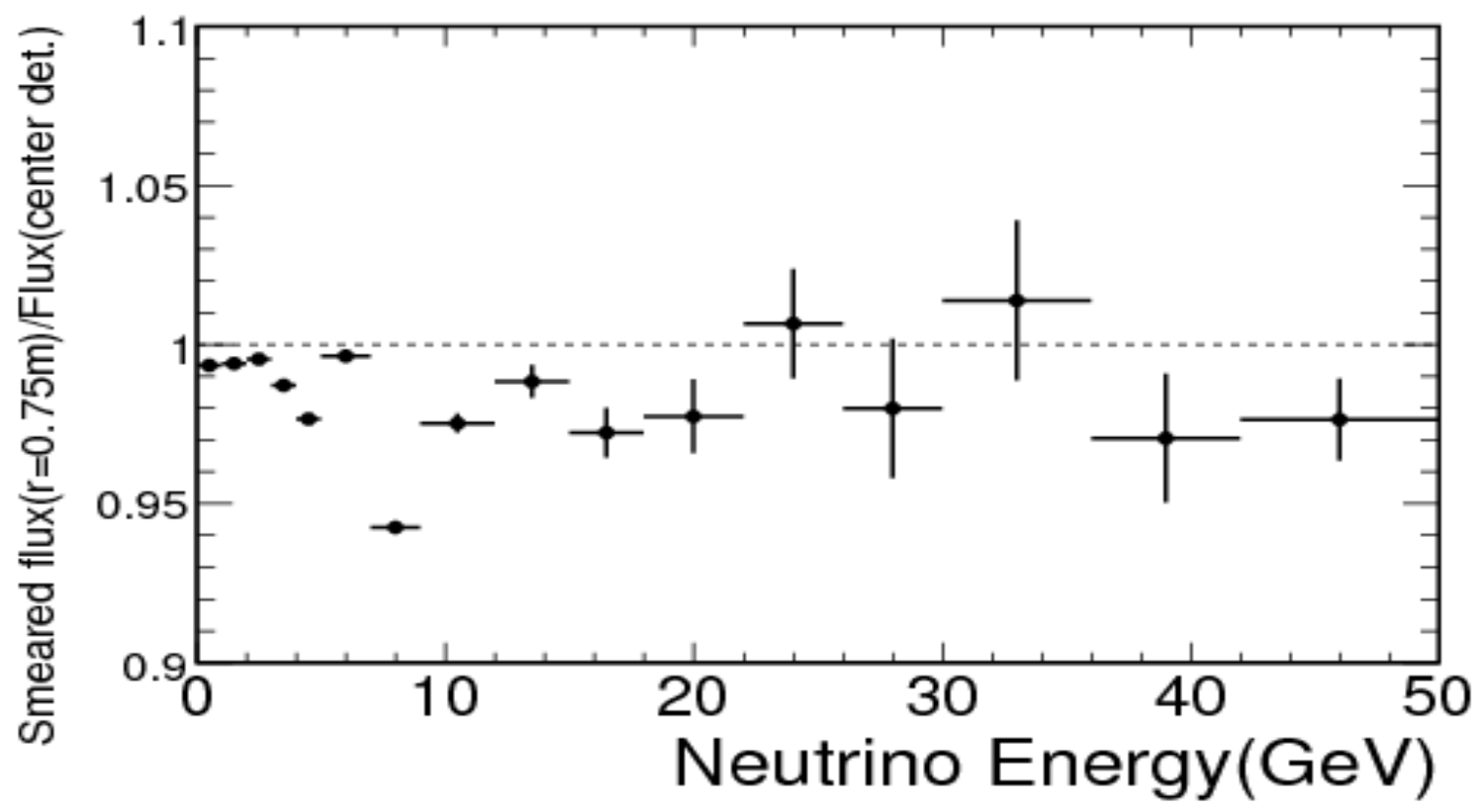

Figure 7.10: Ratio of smeared MC flux over a circular area of radius $0.75 \mathrm{~m}$ to the flux at the center of the detector. 


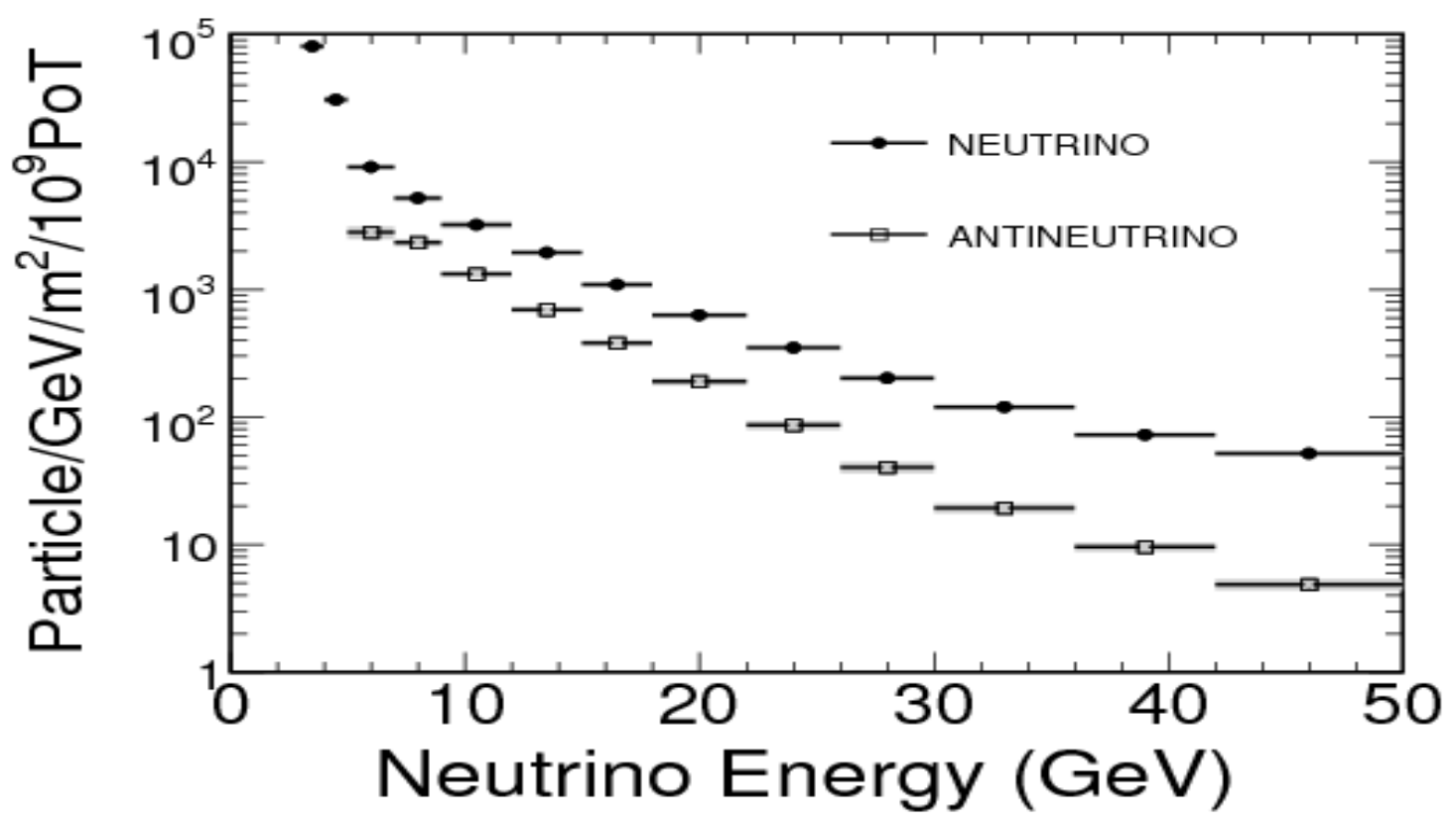

Figure 7.11: Extracted flux for neutrinos(a) and antineutrinos(b) as a function of neutrino energy. The error bars(in black) show the statistical error. The shaded boxes show the statistical and systematic uncertainties added in quadrature. 


\begin{tabular}{|c|c|c|c|c|}
\hline$E$ bin & $v$ Flux & Error & $\bar{v}$ Flux & Error \\
\hline \hline$(\mathrm{GeV})$ & \multicolumn{3}{|c|}{ Particle/GeV $/ \mathrm{m}^{2} / 10^{9} \mathrm{PoT}$} \\
\hline $3-4$ & $80.5 \times 10^{3}$ & $5.02 \times 10^{3}$ & - & - \\
\hline $4-5$ & $30.6 \times 10^{3}$ & $2.33 \times 10^{3}$ & - & - \\
\hline $5-7$ & $9.07 \times 10^{3}$ & 506 & $2.80 \times 10^{3}$ & 325 \\
\hline $7-9$ & $5.18 \times 10^{3}$ & 334 & $2.32 \times 10^{3}$ & 164 \\
\hline $9-12$ & $3.21 \times 10^{3}$ & 210 & $1.32 \times 10^{3}$ & 82.3 \\
\hline $12-15$ & $1.94 \times 10^{3}$ & 98.1 & 689 & 40.4 \\
\hline $15-18$ & $1.09 \times 10^{3}$ & 63.3 & 379 & 23.7 \\
\hline $18-22$ & 629 & 36.6 & 190 & 14.1 \\
\hline $22-26$ & 348 & 20.1 & 86.3 & 7.67 \\
\hline $26-30$ & 200 & 12.5 & 40.1 & 3.89 \\
\hline $30-36$ & 119 & 6.63 & 19.3 & 1.83 \\
\hline $36-42$ & 72.2 & 3.84 & 9.56 & 0.85 \\
\hline $42-50$ & 51.6 & 2.78 & 4.86 & 0.49 \\
\hline
\end{tabular}

Table 7.2: Absolute neutrino(second column) and antineutrino(fourth column) flux as a function of energy. The third and fifth columns show the error(systematic uncertainty and statistical uncertainty added in quadrature) on the extracted flux. 

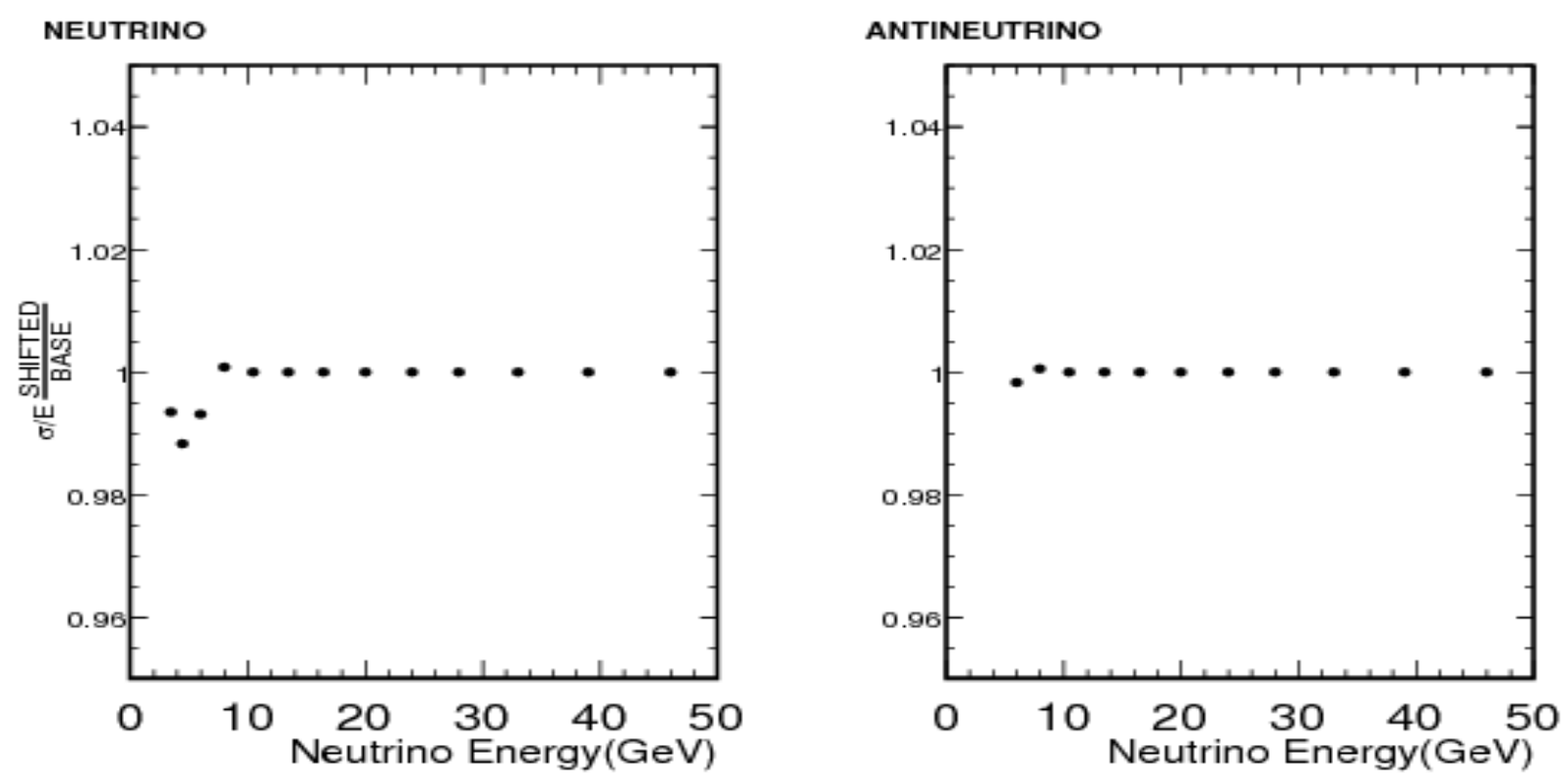

Figure 7.12: Effect of modifying the $v<1 \mathrm{GeV}$ cut to $v<0.75 \mathrm{GeV}$ on the measured cross section.

\subsection{CROSS SECTION MEASUREMENT}

The cross section is extracted by dividing the selected CC sample (after applying the acceptance, background and isoscalar corrections) with the measured flux in each energy bin. Fig. 6.12 shows the acceptance correction and the details have been discussed in section 6.4. Table 7.3 shows the selected events in the neutrino and antineutrino sample and the acceptance correction applied to each energy bin.

The size of the contribution from contamination in the selected cross section sample is shown in Fig. 7.14. The NC contamination is small for both neutrinos and antineutrinos $(<2 \%)$. The increase with energy is due to the contribution from high-inelasticity events in which the primary track is mis-identified or perturbed by extra hits. The wrong sign contamination is small $(\lesssim 0.3 \%)$ in the neutrino sample but sizeable in the antineutrino sample, up to 5\% at high energy. The increasing contamination at high energy is caused by high energy muons which bend little in the 


\begin{tabular}{|c|c|c|c|c|c|}
\hline Bin & Energy Range & Events in $v$ sample & Acceptance Corr. & Events in $\bar{v}$ sample & Acceptance Corr. \\
\hline \hline 1 & $3-4 \mathrm{GeV}$ & 379640 & $2.2 \pm 0.003$ & - & - \\
\hline 2 & $4-5 \mathrm{GeV}$ & 244697 & $1.61 \pm 0.003$ & - & - \\
\hline 3 & $5-7 \mathrm{GeV}$ & 247595 & $1.21 \pm 0.002$ & 22202 & $1.81 \pm 0.012$ \\
\hline 4 & $7-9 \mathrm{GeV}$ & 168334 & $1.37 \pm 0.004$ & 26416 & $1.59 \pm 0.010$ \\
\hline 5 & $9-12 \mathrm{GeV}$ & 212284 & $1.32 \pm 0.003$ & 33740 & $1.46 \pm 0.008$ \\
\hline 6 & $12-15 \mathrm{GeV}$ & 171607 & $1.26 \pm 0.004$ & 24456 & $1.38 \pm 0.009$ \\
\hline 7 & $15-18 \mathrm{GeV}$ & 127021 & $1.22 \pm 0.004$ & 16613 & $1.34 \pm 0.010$ \\
\hline 8 & $18-22 \mathrm{GeV}$ & 116728 & $1.19 \pm 0.004$ & 14257 & $1.32 \pm 0.011$ \\
\hline 9 & $22-26 \mathrm{GeV}$ & 79138 & $1.19 \pm 0.005$ & 8271 & $1.29 \pm 0.013$ \\
\hline 10 & $26-30 \mathrm{GeV}$ & 54681 & $1.18 \pm 0.006$ & 4928 & $1.24 \pm 0.016$ \\
\hline 11 & $30-36 \mathrm{GeV}$ & 56741 & $1.13 \pm 0.005$ & 4328 & $1.21 \pm 0.017$ \\
\hline 12 & $36-42 \mathrm{GeV}$ & 41116 & $1.13 \pm 0.006$ & 2658 & $1.16 \pm 0.023$ \\
\hline 13 & $42-50 \mathrm{GeV}$ & 41437 & $1.19 \pm 0.006$ & 2011 & $1.14 \pm 0.027$ \\
\hline
\end{tabular}

Table 7.3: Raw number of events in energy bins for the neutrino and antineutrino cross section sample and the acceptance correction applied to each sample. The second(neutrino) and fourth(antineutrino) columns show the number of selected events and the third(neutrino) and fifth(antineutrino) columns show the acceptance correction. 
magnetic field and by high inelasticity events where the reconstruction package mistakenly picks up hits from high energy showers. Fig. 7.15 shows the fractional contamination as a function of inelasticity.

The cross section measurement will be compared to measurements on isoscalar targets from other experiments. Fig. 7.13 shows a correction that is applied to the cross section sample to correct for non-isoscalarity of the MINOS iron target.

In Eq. 1.37 it is shown that the structure function $F_{2}$ is equal for neutrinos and antineutrinos. But due to the presence of quark mixing the difference in structure function is given by

$$
\Delta F_{2}\left(x, Q^{2}\right)=F_{2}^{v}\left(x, Q^{2}\right)-F_{2}^{\bar{v}}\left(x, Q^{2}\right)=-\frac{1+R}{1+4 M^{2} x^{2} / Q^{2}} \times V_{u s} \times \frac{1}{2}\left(u_{V}+d_{V}\right),
$$

where, $V_{u s}$ is the up and strange quark mixing term, $u_{V}$ and $d_{V}$ are the valence up and down quark PDFs. In absence of quark mixing the same normalization constant applies to antineutrinos. We apply an additional normalization computed from the cross section model (about $1 \%$ for $v<1$ $\mathrm{GeV}, 2.6 \%$ for $v<2 \mathrm{GeV}$ and $3.8 \%$ for the $v<5 \mathrm{GeV}$ sample) to the antineutrino sample to account for this quark mixing term. The effect of varying the model parameters on this correction factor was evaluated and was found to be very small. In addition, the $V_{u s}$ parameter was varied by $\pm 1 \sigma(0.2257 \pm 0.0021)$ and it also had a small effect.

The procedure described in section 7.1 gives the energy dependence of the cross section. To normalize the cross section, the neutrino world average cross section value in the energy range 30$50 \mathrm{GeV}$ on an isoscalar target found to be $(0.675 \pm 0.009) \times 10^{-38} \mathrm{~cm}^{2} / \mathrm{GeV}$ [3] is used. The error on the normalization constant is the error on the MINOS weighted average cross section between $30-50 \mathrm{GeV} .{ }^{1}$ This error is added in quadrature to the $1.3 \%$ error on the world average cross section

\footnotetext{
${ }^{1}$ The error on the weighted mean is taken to be $\sigma=\sqrt{\left(\frac{1}{\Sigma w_{i}}\right) \times \frac{\chi^{2}}{n-1}}$, where $\mathrm{n}$ is the number of bins( 3 in our case). This underestimates the error because we have only three points and therefore the reduced $\chi^{2}(0.41 / 2$ for the $v<1$ $\mathrm{GeV}$ sample) is very small. The more appropriate error on the normalization constant should be $\sqrt{1 / \Sigma w_{i}}$. The overall effect of this change is very small because the error on normalization constant is small compared to the systematic uncertainties. The total normalization error is obtained by adding the error on the weighted mean in quadrature to the $1.3 \%$ error on the world measurement. The change in percentage error on the normalization constant is listed in the table below.

\begin{tabular}{|c|c|c|}
\hline \multirow{2}{*}{$v \operatorname{cut}(\mathrm{GeV})$} & \multicolumn{2}{|c|}{$\%$ normalization error } \\
\cline { 2 - 3 } & new & old \\
\hline$v<1$ & 2.32 & 1.66 \\
$v<2$ & 1.99 & 1.45 \\
$v<5$ & 1.69 & 1.33 \\
\hline
\end{tabular}
}




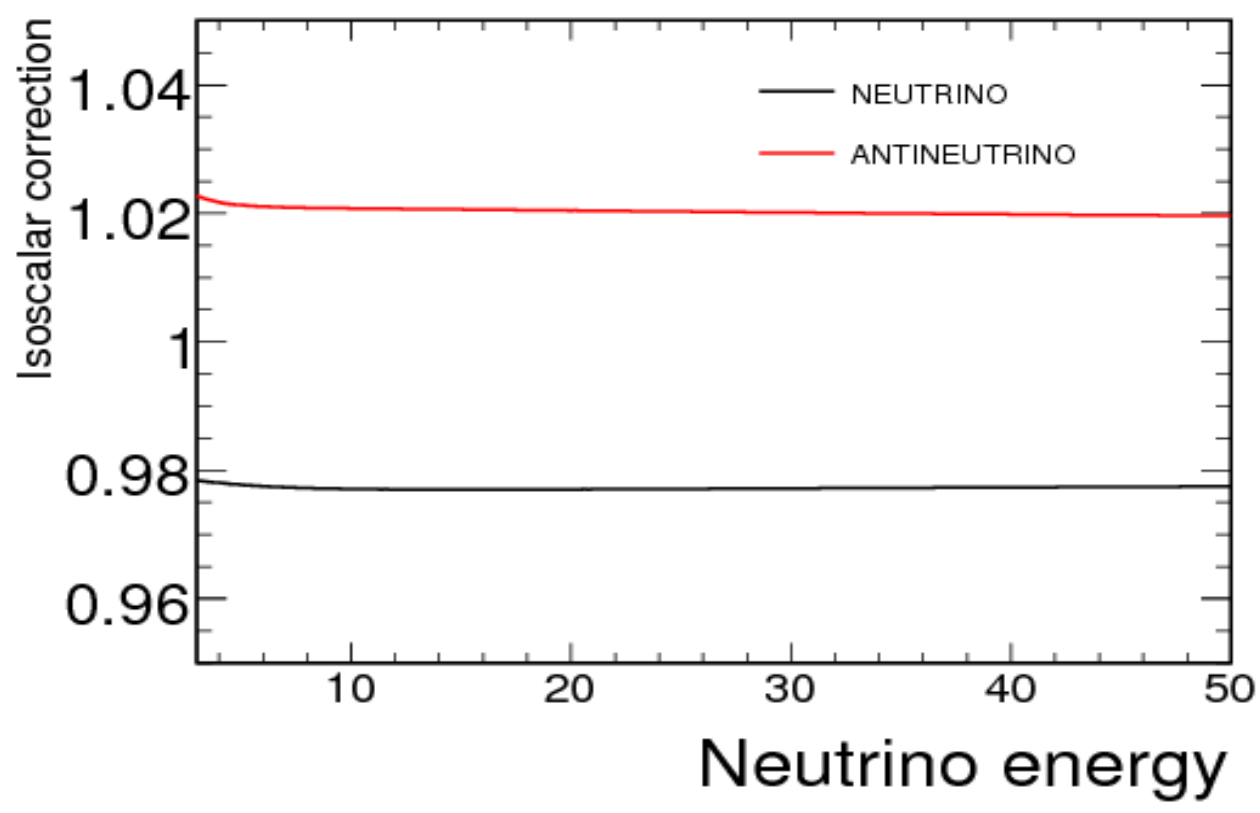

Figure 7.13: Isoscalar correction applied to the neutrino and antineutrino cross section sample. The black line shows the correction for neutrino and the red line shows the correction for antineutrino. 
to obtain the total normalization error.

After extracting the initial flux and cross section, the procedure is iterated by re-extracting the cross section with the extracted flux (Fig. 7.8). This removes any effect of the simulated flux on the analysis. The change in cross section between the first iteration and the one extracted with the default simulated flux is less than $0.5 \%$ (averaged over all data points), since this effect is small we have performed only one iteration. The main effect of changing the flux is in the acceptance correction, which mostly cancels in the flux and cross section samples.

Fig. 7.16 shows the extracted energy dependence of the $v_{\mu} N \mathrm{CC} \sigma / E$ on an isoscalar target. The cross section values are calculated at the energy bin average and divided by this energy value. The measured cross section has a linear energy dependence for energies above $20 \mathrm{GeV}$. The low energy region $(<20 \mathrm{GeV})$ shows an indication of a rise with decreasing energy as expected due to the presence of quasi-elastic and resonance interactions. Table 7.4 summarizes the neutrino cross section results. The systematic uncertainties are discussed in section 7.5.

The energy dependence of the $\bar{v}_{\mu} N \mathrm{CC} \sigma / E$ on an isoscalar target is shown in Fig. 7.17. The cross section has a linear energy dependence above $20 \mathrm{GeV}$ and decreases with energy below 20 $\mathrm{GeV}$. Table 7.5 summarizes the antineutrino cross section results. Interpretation of the results is given in chapter 8.

Fig. 7.18 shows the ratio of the $\bar{v}_{\mu} N \mathrm{CC}$ to $v_{\mu} N \mathrm{CC}$ inclusive cross section as a function of energy. The ratio can be robustly determined because many of the experimental uncertainties are reduced due to partial cancellation. The average of this ratio in the $30-200 \mathrm{GeV}$ range calculated from other experiments has a value of $0.504 \pm 0.003$, which agrees with the MINOS average in the energy range $30-50 \mathrm{GeV}(0.489 \pm 0.012)$. The MINOS result also populates the lower energy region where data from other experiments is sparse.

\subsection{SYSTEMATIC UNCERTAINTIES}

We have considered the following sources of systematic uncrtainties in the measurement: muon and hadronic energy scales, the effect of final state interactions on the measured energy, NC contamination, wrong-sign contamination (antineutrino sample only), understanding of the detector 

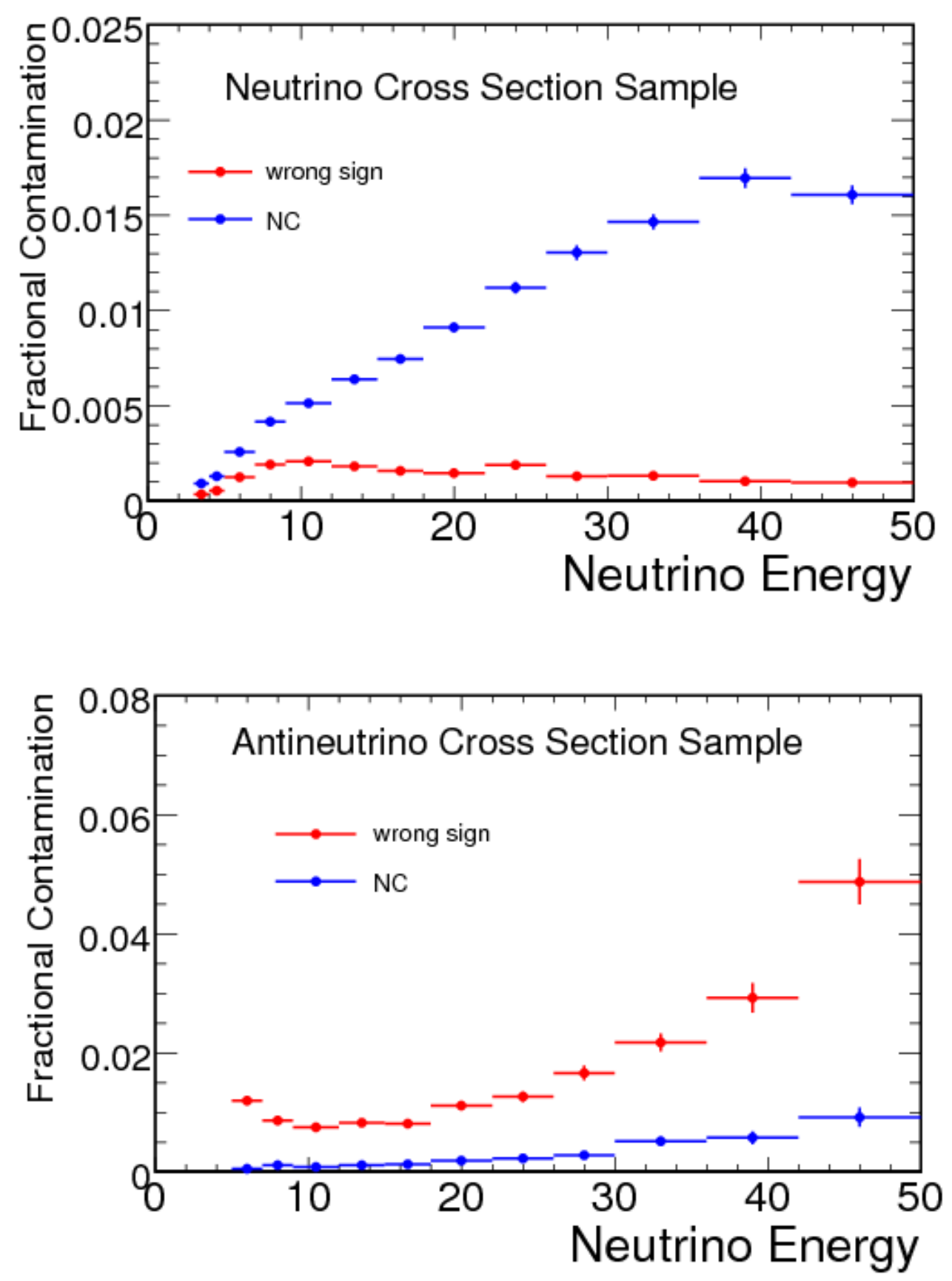

Figure 7.14: Contamination from NC and wrong-sign events as a function of energy in the neutrino(a) and antineutrino(b) selected charged current sample as calculated from the MC event sample. The error bars show the statistical error only. 


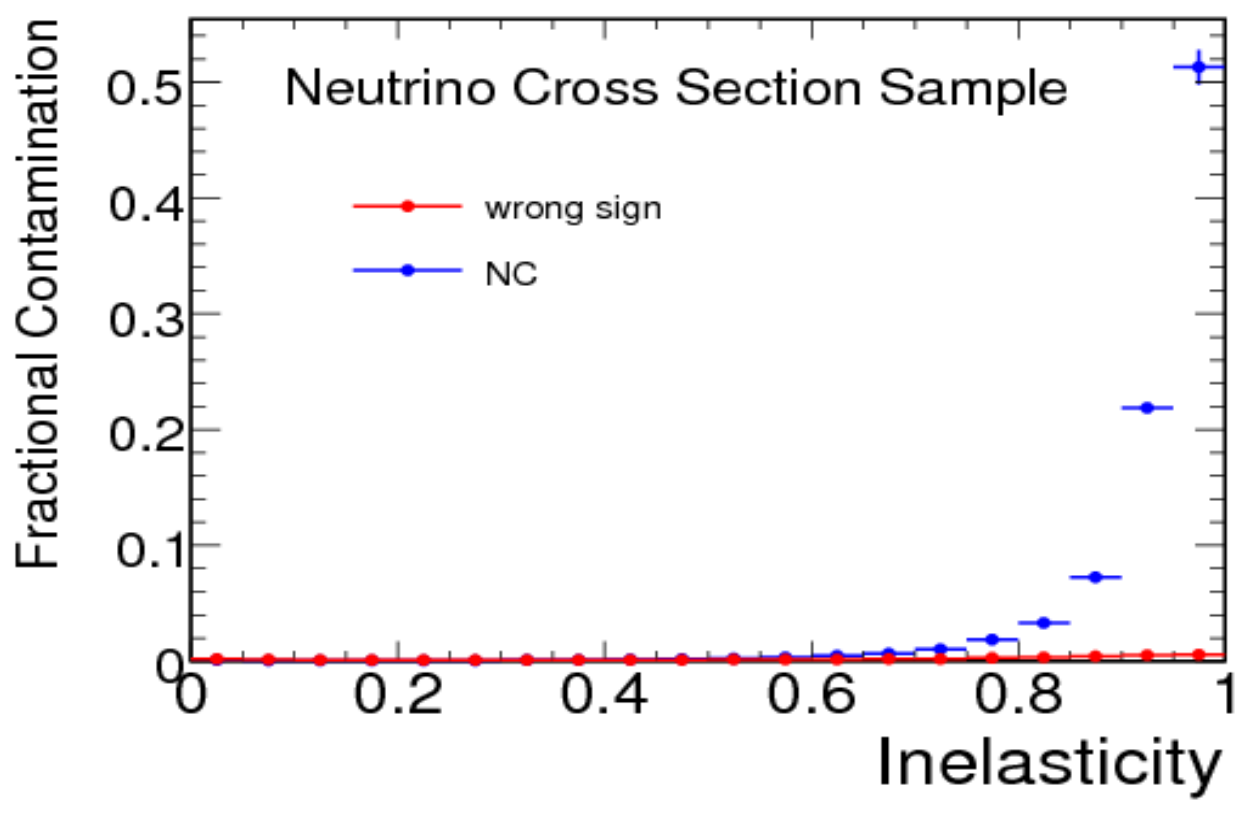

(a)

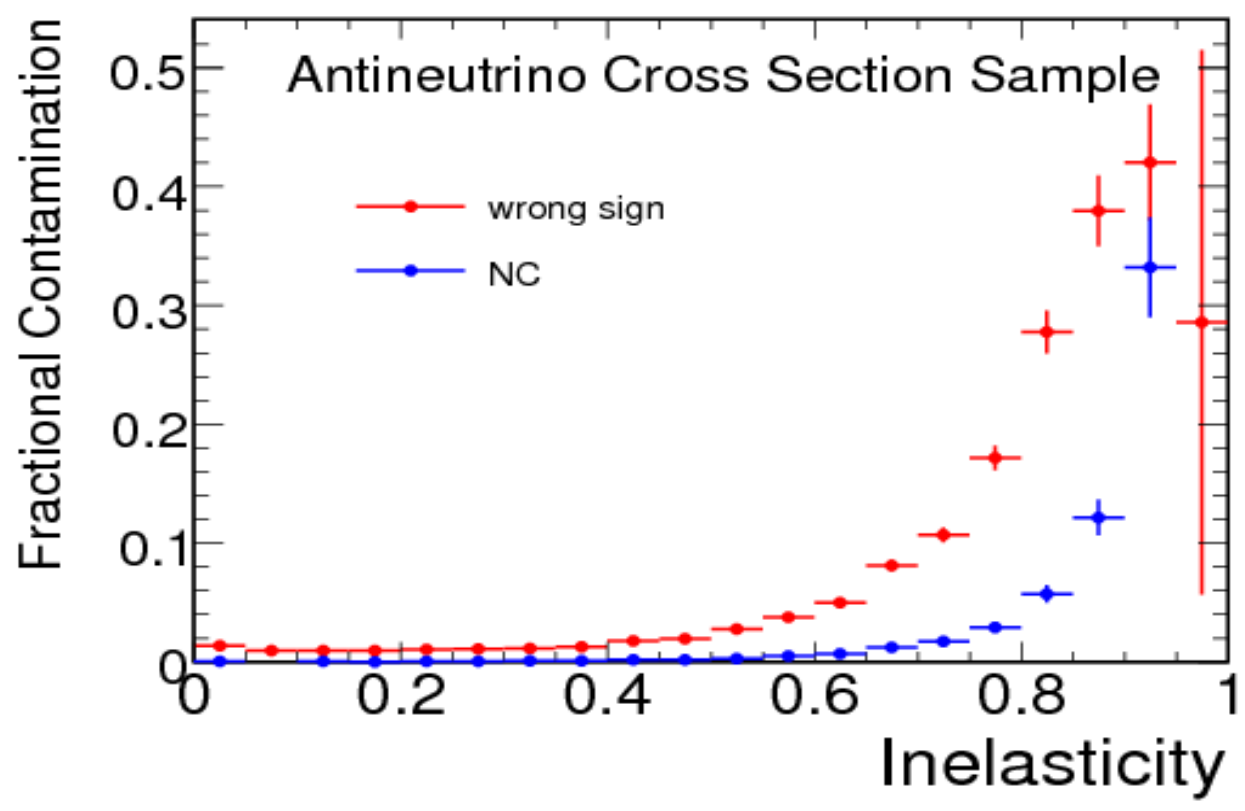

(b)

Figure 7.15: Contamination from NC and wrong-sign events as a function of inelasticity in the neutrino(a) and antineutrino(b) selected charged current sample as calculated from the MC event sample. The error bars show the statistical error only. 


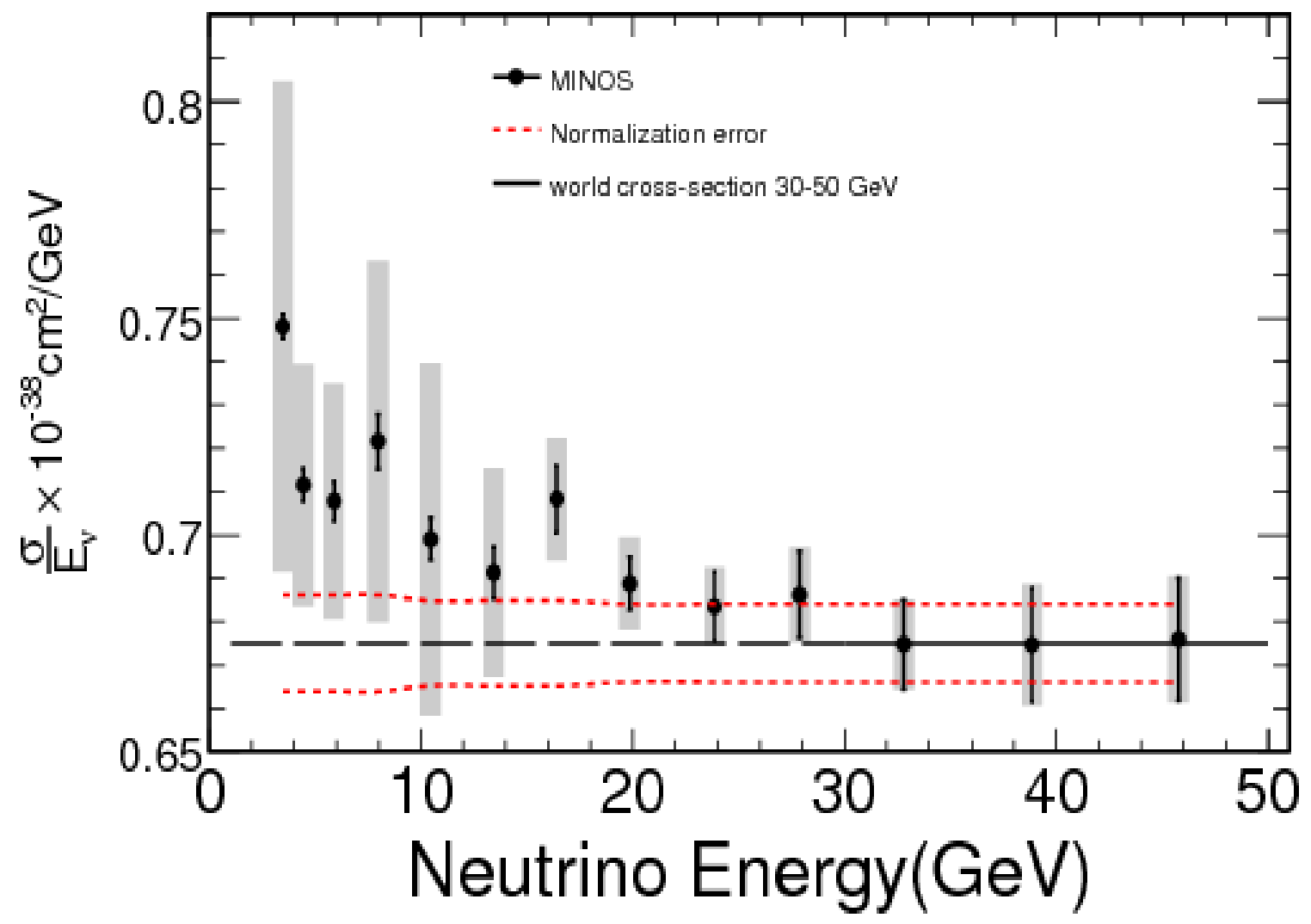

Figure 7.16: Neutrino charged-current inclusive cross section as a function of energy for an isoscalar iron target. The black error bars show the statistical uncertainty and the shaded boxes show the statistical and systematic uncertainties added in quadrature. The dotted red band shows the normalization error that is about $1.5 \%$. The solid black line shows the world cross section value $\left(0.675 \times 10^{-38} \mathrm{~cm}^{2} / \mathrm{GeV}\right)$ in the $30-50 \mathrm{GeV}$ range and the dashed black line shows this value extrapolated to lower energies. The neutrino cross section is normalized to this value. 


\begin{tabular}{|c|c|c|c|c|c|c|}
\hline$E$ bin & $E_{\text {av. }}$ & $\sigma / E$ & stat. error & sys. error & norm. error & total error \\
\hline \hline \multicolumn{5}{|c|}{$(\mathrm{GeV})$} & \multicolumn{5}{|c|}{$\left(10^{-38} \mathrm{~cm}^{2} / \mathrm{GeV}\right)$} \\
\hline $3-4$ & 3.48 & 0.748 & 0.003 & 0.057 & 0.012 & 0.058 \\
\hline $4-5$ & 4.45 & 0.711 & 0.004 & 0.028 & 0.012 & 0.030 \\
\hline $5-7$ & 5.89 & 0.708 & 0.005 & 0.027 & 0.012 & 0.030 \\
\hline $7-9$ & 7.97 & 0.722 & 0.006 & 0.041 & 0.012 & 0.043 \\
\hline $9-12$ & 10.45 & 0.699 & 0.005 & 0.040 & 0.010 & 0.042 \\
\hline $12-15$ & 13.43 & 0.691 & 0.006 & 0.023 & 0.010 & 0.026 \\
\hline $15-18$ & 16.42 & 0.708 & 0.008 & 0.012 & 0.010 & 0.018 \\
\hline $18-22$ & 19.87 & 0.689 & 0.006 & 0.009 & 0.009 & 0.014 \\
\hline $22-26$ & 23.88 & 0.683 & 0.008 & 0.005 & 0.009 & 0.013 \\
\hline $26-30$ & 27.89 & 0.686 & 0.010 & 0.005 & 0.009 & 0.014 \\
\hline $30-36$ & 32.81 & 0.675 & 0.010 & 0.002 & 0.009 & 0.014 \\
\hline $36-42$ & 38.87 & 0.675 & 0.013 & 0.005 & 0.009 & 0.017 \\
\hline $42-50$ & 45.77 & 0.676 & 0.014 & 0.004 & 0.009 & 0.017 \\
\hline
\end{tabular}

Table 7.4: Summary of neutrino cross section results. The second column shows the energy bin average. The fourth, fifth and sixth columns show the statistical, systematic and normalization uncertainties respectively. The last column shows the total error, which is obtained by adding these three uncertainties in quadrature. 


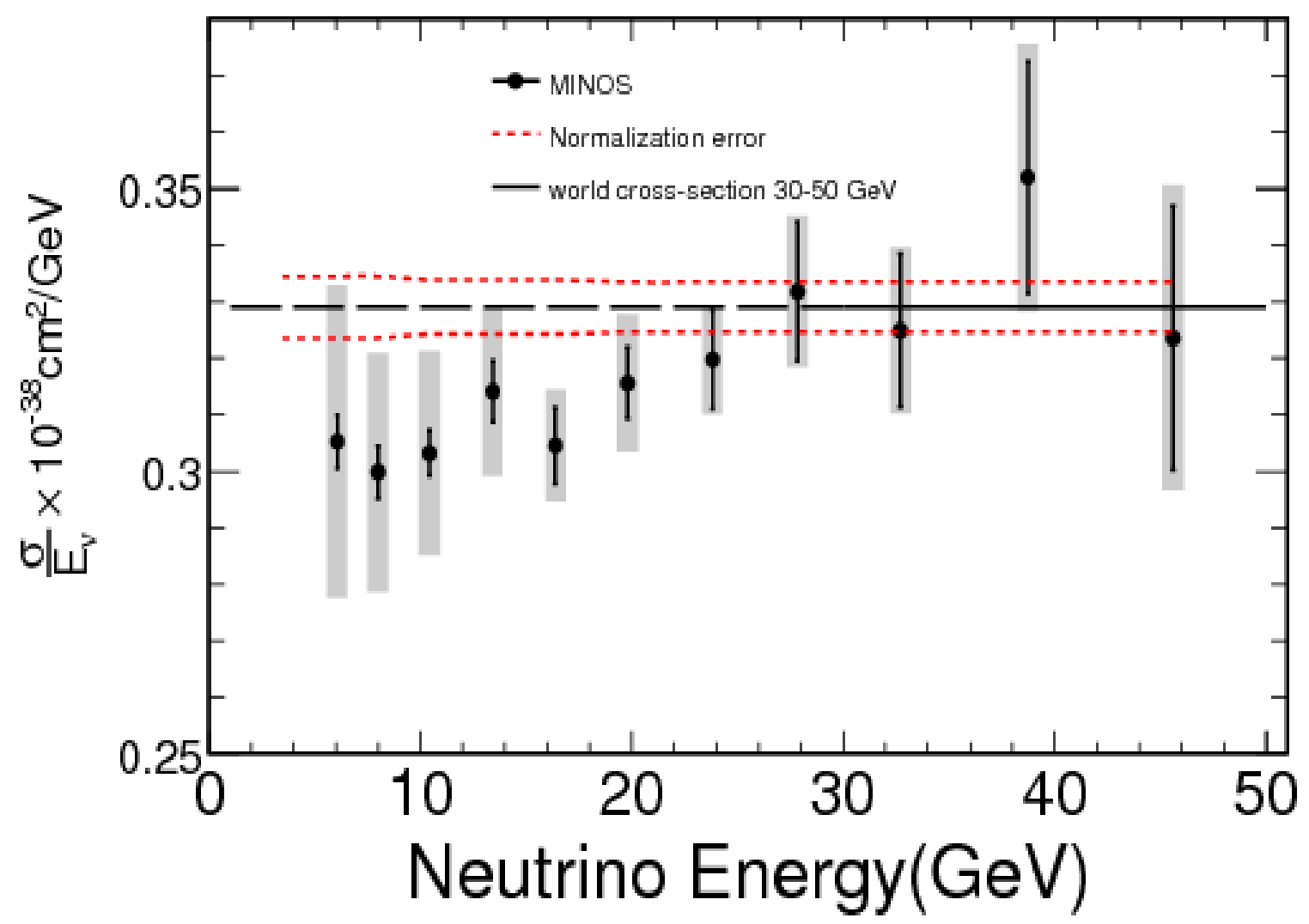

Figure 7.17: Antineutrino charged-current inclusive cross section as a function of energy for an isoscalar iron target. The black error bars show the statistical uncertainty and the shaded boxes show the statistical and systematic uncertainties added in quadrature. The dotted red band shows the error on MINOS normalization constant and the error on world cross section data added in quadrature(about $1.5 \%$ ). The solid black line shows the world cross section value $(0.329$ $\times 10^{-38} \mathrm{~cm}^{2} / \mathrm{GeV}$ ) in the $30-50 \mathrm{GeV}$ range and the dashed black line shows this value extrapolated to lower energies. 


\begin{tabular}{|c|c|c|c|c|c|c|}
\hline$E$ bin & $E_{\text {av. }}$ & $\sigma / E$ & stat. error & sys. error & norm. error & total error \\
\hline \hline \multicolumn{6}{|c|}{$(\mathrm{GeV})$} & \multicolumn{5}{|c|}{$\left(10^{-38} \mathrm{~cm}^{2} / \mathrm{GeV}\right)$} \\
\hline $5-7$ & 6.07 & 0.305 & 0.005 & 0.027 & 0.005 & 0.028 \\
\hline $7-9$ & 7.99 & 0.300 & 0.005 & 0.021 & 0.005 & 0.022 \\
\hline $9-12$ & 10.43 & 0.303 & 0.004 & 0.018 & 0.004 & 0.019 \\
\hline $12-15$ & 13.42 & 0.314 & 0.005 & 0.014 & 0.005 & 0.016 \\
\hline $15-18$ & 16.41 & 0.304 & 0.007 & 0.007 & 0.004 & 0.011 \\
\hline $18-22$ & 19.82 & 0.316 & 0.006 & 0.010 & 0.004 & 0.013 \\
\hline $22-26$ & 23.82 & 0.320 & 0.009 & 0.004 & 0.004 & 0.011 \\
\hline $26-30$ & 27.84 & 0.332 & 0.012 & 0.005 & 0.004 & 0.014 \\
\hline $30-36$ & 32.72 & 0.325 & 0.014 & 0.006 & 0.004 & 0.015 \\
\hline $36-42$ & 38.74 & 0.352 & 0.021 & 0.011 & 0.005 & 0.024 \\
\hline $42-50$ & 45.61 & 0.324 & 0.023 & 0.014 & 0.004 & 0.027 \\
\hline
\end{tabular}

Table 7.5: Summary of antineutrino cross section results. The second column shows the energy bin average. The fourth, fifth and sixth columns show the statistical, systematic and normalization uncertainties respectively. The last column shows the total error, which is obtained by adding these three uncertainties in quadrature. 


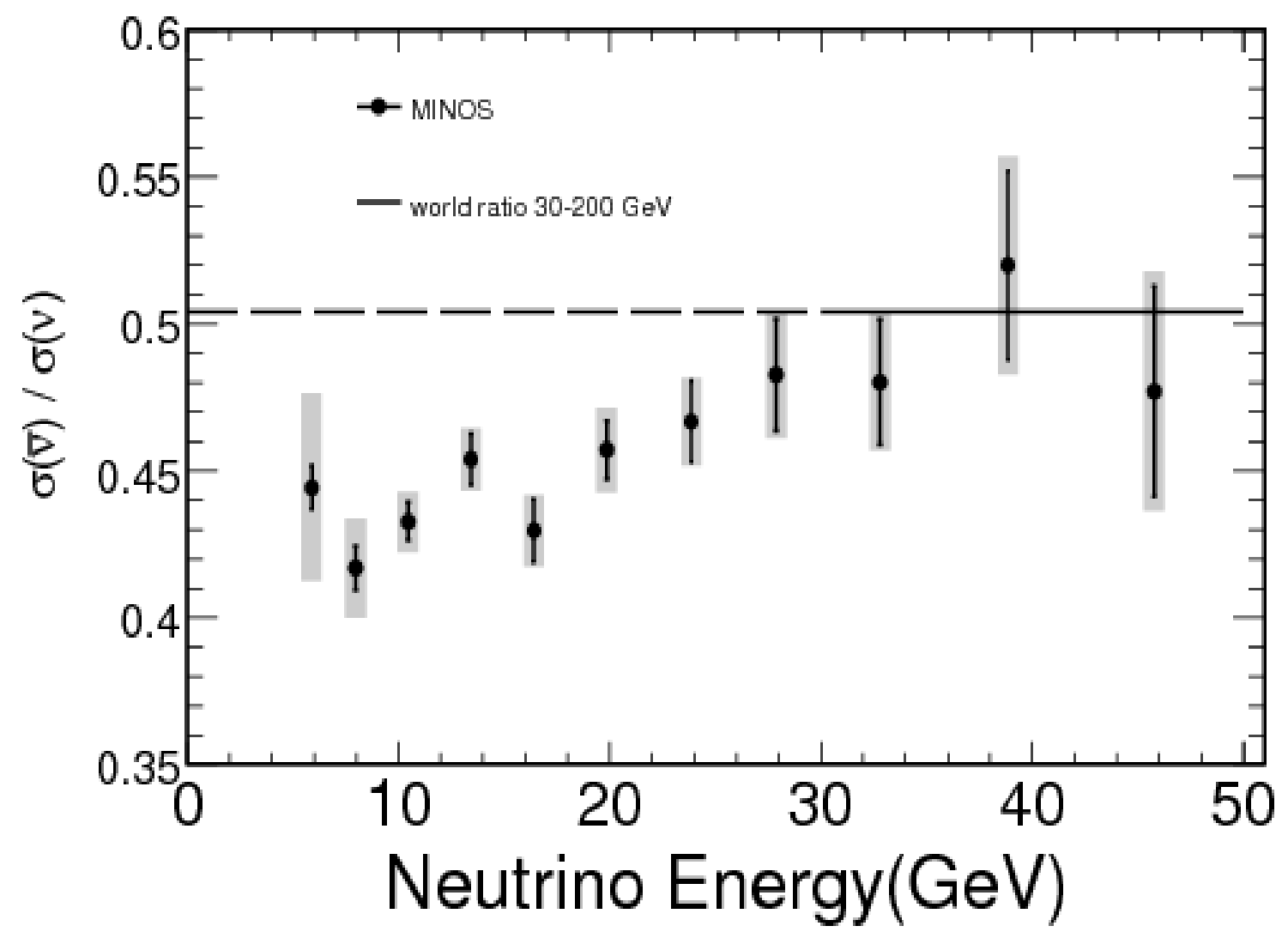

Figure 7.18: Ratio of antineutrino-nucleon to neutrino-nucleon cross section as a function of energy. The black error bars show the statistical uncertainty and the shaded boxes show the statistical and systematic uncertainties added in quadrature. The solid black line shows the value of this ratio from other experiments between $30-200 \mathrm{GeV}$ at $0.504 \pm 0.003$ [3]. 
and event reconstruction, and cross section modeling. Each systematic uncertainty is evaluated independently and propagated through the entire analysis which includes a recalculation of the absolute normalization of the flux. Many systematic effects cause similar changes to the cross section and flux samples and therefore often partially cancel in the measured cross section (see Eq. 7.1 ).

\subsubsection{Energy scale}

The largest uncertainty comes from knowledge of the absolute hadronic and muon energy scales. The uncertainty on the range momentum is $2 \%$ and on momentum from curvature is $4 \%$ (section 3.3). The uncertainty on the hadronic energy scale is 5.6\% (section 3.2). Fig. 7.19 and Fig. 7.20 show the effect of muon and hadronic energy scale uncertainties on the extracted flux and cross section respectively.

The muon energy scale is more important for the flux sample than for the cross section sample because a larger fraction of the neutrino energy per event is carried by the muon in the former. When the energy scale is shifted, the data sample distribution with the shifted scale crosses the data sample distribution with the unshifted scale at certain energy values. Fig. 7.19 shows that for the muon energy scale (black line) the inflection points for the neutrino(antineutrino) flux are at 6 and $14 \mathrm{GeV}(12 \mathrm{GeV})$. The energy dependence of the muon energy scale uncertainty in Fig. 7.20(black line) has a non-trivial shape because of the effect of both the flux sample and the cross section sample.

The uncertainty on the hadronic energy is determined by shifting the $E_{H A D}$ scale in data by 5.6\%. Fig. 7.19(red line) shows that the effect of shifting the hadronic energy scale on the extracted flux is mostly independent of energy. This is due to the fact that shifting the hadronic energy scale is basically shifting the $v$ cut that we apply on our flux sample. Fig. 7.20(red line) shows the effect on the extracted $\sigma / E$.

As described in section 5.6, final state interactions affect the measured hadronic energy and must be modeled. To determine the uncertainty from the intranuclear rescattering model, each of the contributing cross sections(pion and nucleon) are changed by $\pm \sigma$ and the effect on the reconstructed shower energy is evaluated. In addition, uncertainties in the modeling of the formation zone is also accounted for. Adding all these contributions in quadrature gives an energy dependent 
correction to the hadronic energy scale shown in Fig. 7.21. This uncertainty is treated in the same way as the hadronic energy scale uncertainty and the effect on the extracted flux and cross section is shown in Fig. 7.19 and 7.20 respectively.

\subsubsection{Cross section model}

The cross section model has been discussed in chapter 5. We shift the model parameters by their uncertainty and study the effect on the analysis. The uncertainties in the cross section modeling will affect the $v$ dependence of the cross section (Eq. 7.9) that could affect the flux extraction. In addition, it also affects the acceptance correction since this is determined from MC simulation.

We account for the uncertainties in the axial mass of the QEL and resonance cross sections, $M_{A, Q E L}(0.99 \pm 0.15)$ and $M_{A, R E S}(1.12 \pm 0.17) \mathrm{GeV} / \mathrm{c}^{2}$. The uncertainty in the DIS model parameters is determined by varying each parameter in the model [5] and studying the effect on the reduced $\chi^{2}$ of the fit to the charged-lepton data. We take the shift that corresponds to a one unit shift in fit $\chi^{2}$ as the uncertainty for each parameter. The effect of $A_{h t}(0.538 \pm 0.134), B_{h t}(0.305 \pm 0.076)$, $C_{V 1 u}(0.291 \pm 0.087)$ and $C_{V 2 u}(0.189 \pm 0.076)$ on the analysis has been evaluated $\left(C_{v 1 d}, C_{v 2 d}\right.$ and $C_{s}$ have very small effect on the $\chi^{2}$ and hence have been neglected).

The direct effect of the cross section model parameters on the flux extraction is very small $(<$ $1 \%$ ) and has been discussed in section 7.2. The effect of model uncertainty on the extracted cross section is at the level of $2 \%$ below $8 \mathrm{GeV}$ for both neutrino and antineutrino cross sections, and is negligible above this energy.

\subsubsection{Acceptance correction}

Section 6.6 described the reverse field dataset that was collected to evaluate the effect of acceptance correction modeling on the analysis. Fig. 7.22 shows the ratio of reverse field to forward field extracted flux. The neutrino flux shows a shift of about $5 \%$ within error bars which is caused by the difference in the flux reweighting function (Fig. 7.8) for the forward field and reverse field. The systematic uncertainty on the extracted flux is taken to be half of this ratio and added in quadrature

to other uncertainties. The uncertainty on the antineutrino flux has been ignored because the ratio is $\sim 1$ within error bars. 


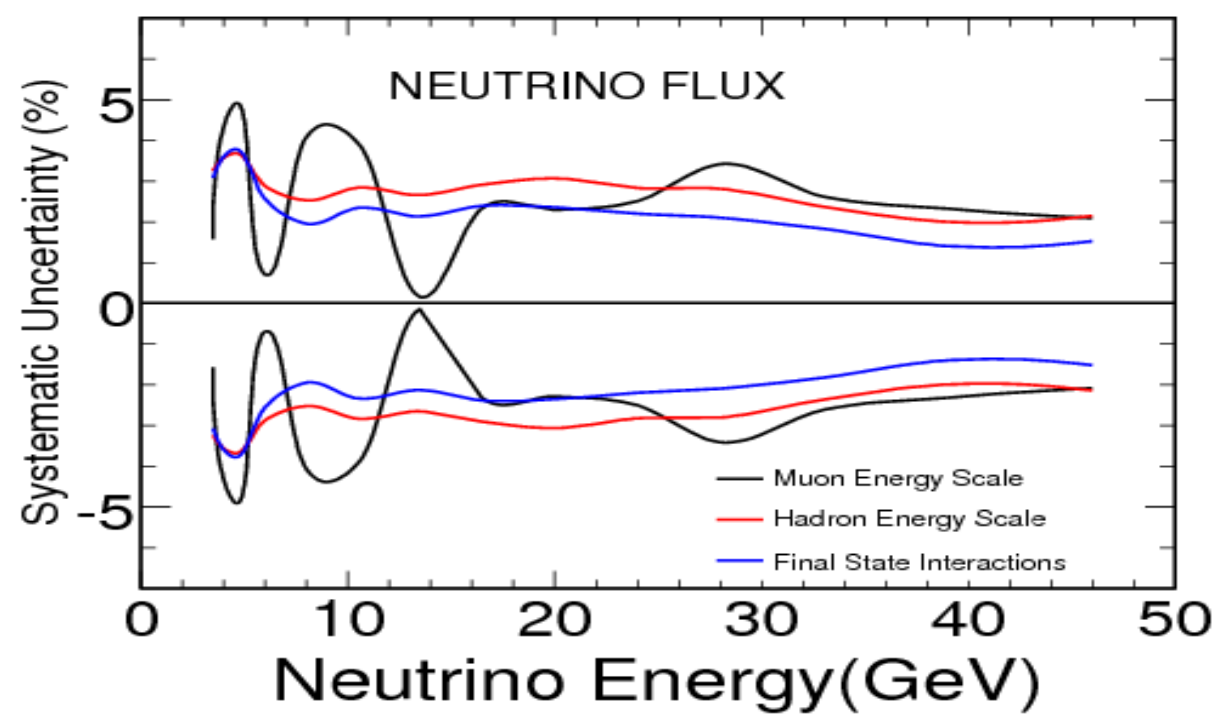

(a)

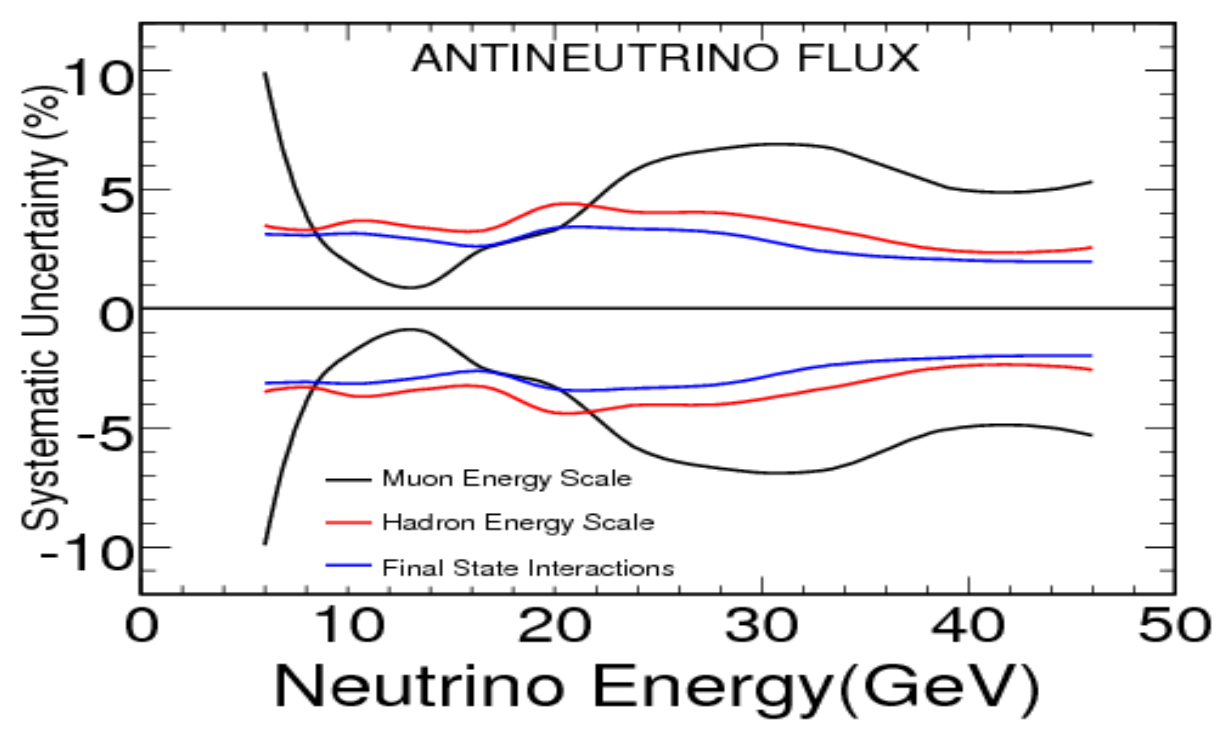

(b)

Figure 7.19: Effect of the energy scale uncertainty on the neutrino(a) and antineutrino(b) extracted flux. The black line shows the effect of shifting the muon energy scale by $2 \%$ for stopping muons and $4 \%$ for exiting muons. The red line shows the effect of shifting the hadronic energy scale by $5.6 \%$ and the blue line shows the uncertainty from final state interactions. 


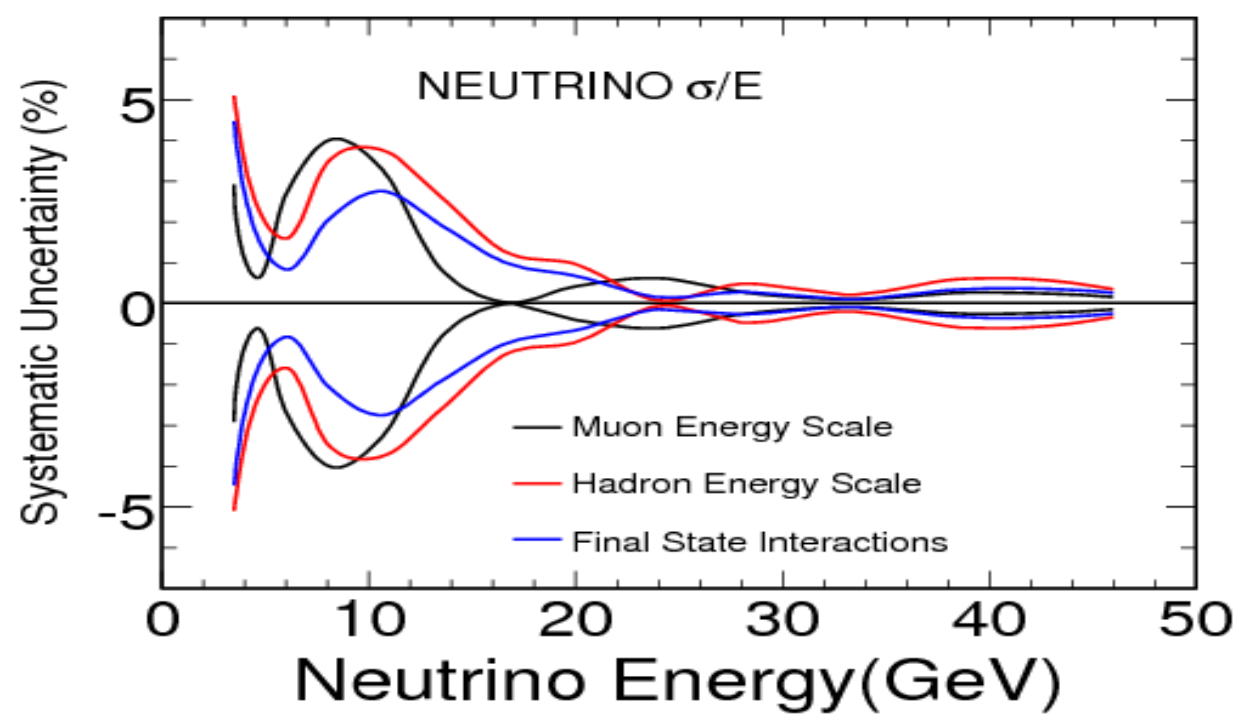

(a)

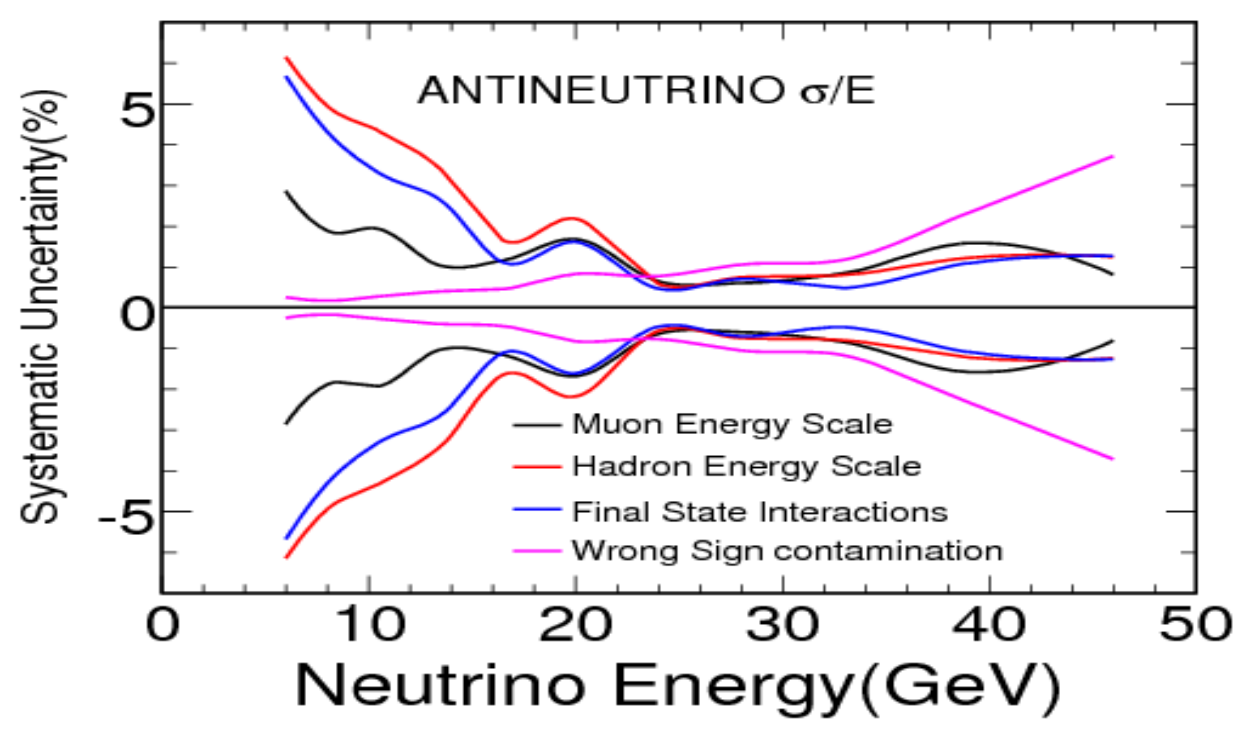

(b)

Figure 7.20: Effect of the energy scale uncertainty on the neutrino(a) and antineutrino(b) extracted cross section. The black line shows the effect of shifting the muon energy scale by $2 \%$ for stopping muons and $4 \%$ for exiting muons. The red line shows the effect of shifting the hadronic energy scale by $5.6 \%$ and the blue line shows the uncertainty from final state interactions. 


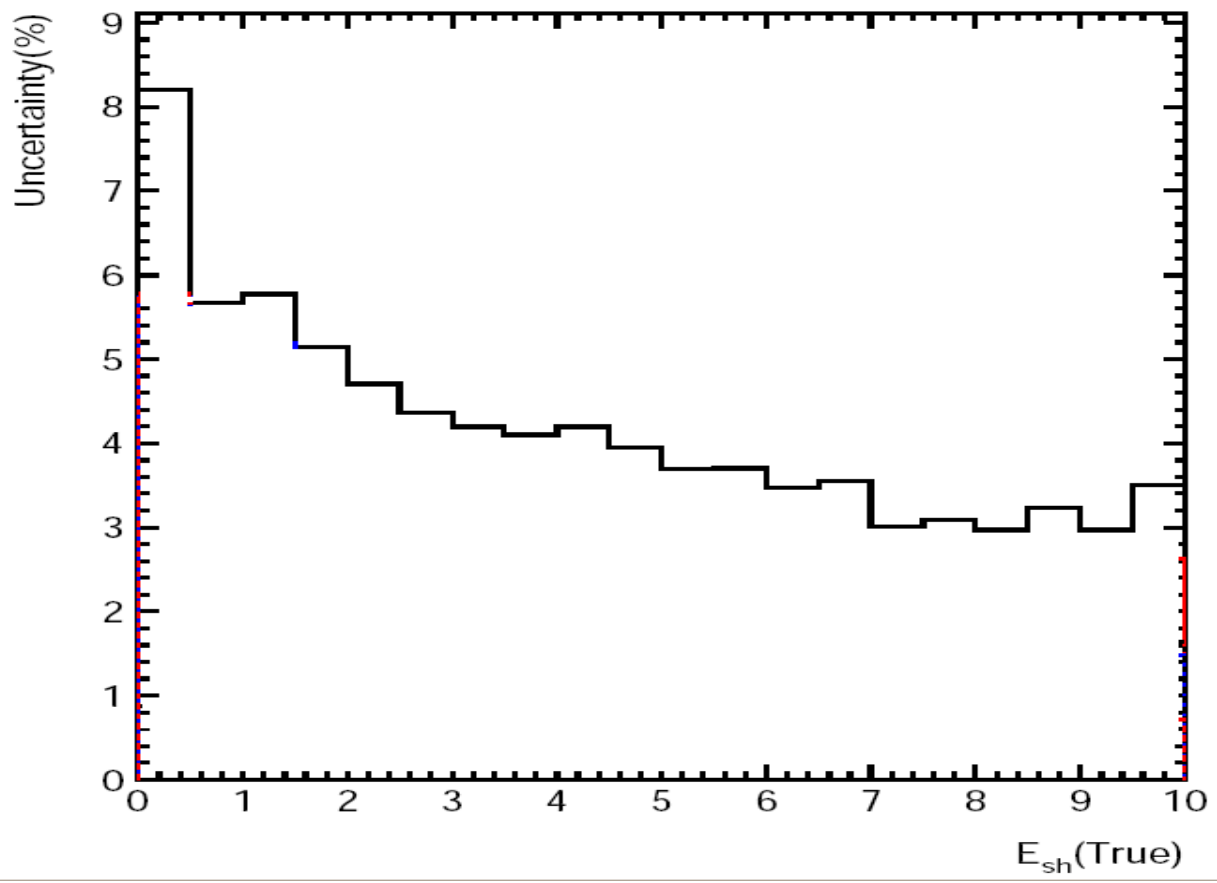

Figure 7.21: Uncertainty in the intranuclear rescattering model as a function of true shower energy.

Fig. 7.23 shows the ratio of reverse field to forward field extracted cross section. We see a difference at the level of $1 \%$ in the extracted cross section from the reversed field and the forward field data. This effect was neglected because this is within the statistical precision of the data set.

\subsubsection{Background}

The uncertainty from our knowledge of the NC contamination is obtained by varying the value of the minimum $E_{\mu}$ cut, which selects the $\mathrm{CC}$ sample, from its nominal value of $1.5 \mathrm{GeV}$ up to 2.0 $\mathrm{GeV}$ and down to $1.0 \mathrm{GeV}$. Result of this study shown in Fig. 7.24 also helps in evaluating the effect of modifying the $E_{\mu}$ cut on the analysis. This change in the $E_{\mu}$ cut either halves the NC contamination $\left(E_{\mu}>2 \mathrm{GeV}\right)$ or doubles it $\left(E_{\mu}>1 \mathrm{GeV}\right)$ as can be seen in Fig. 6.3. The resulting change in $\sigma / E$ is small because we change the $E_{\mu}$ value in the data and MC event sample. This corresponds to a change of less than $1 \%$ which we take to be the NC contamination uncertainty.

The contamination from wrong-sign events is non-negligible only for the antineutrino sam- 

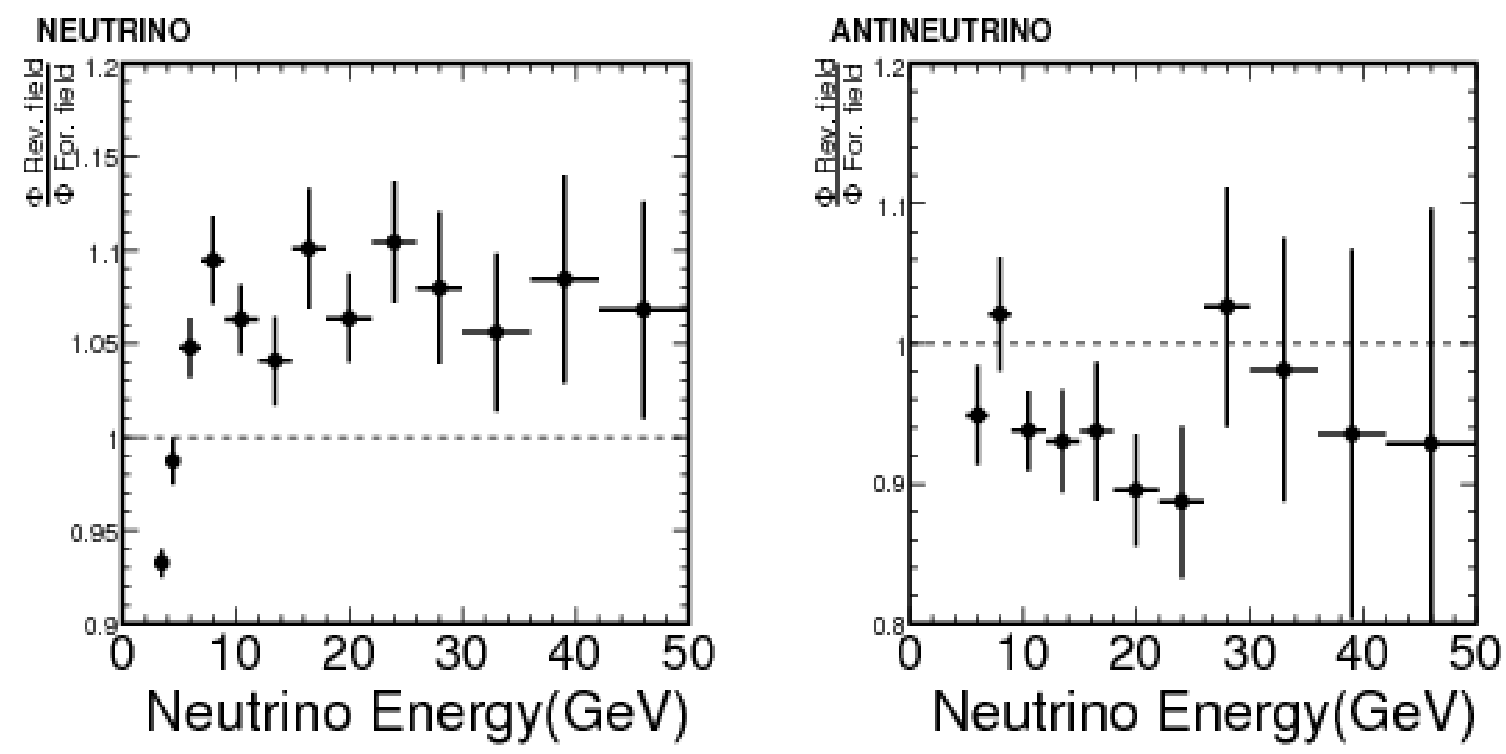

Figure 7.22: Ratio of reverse field to forward field extracted flux for neutrinos(left) and antineutrinos(right).
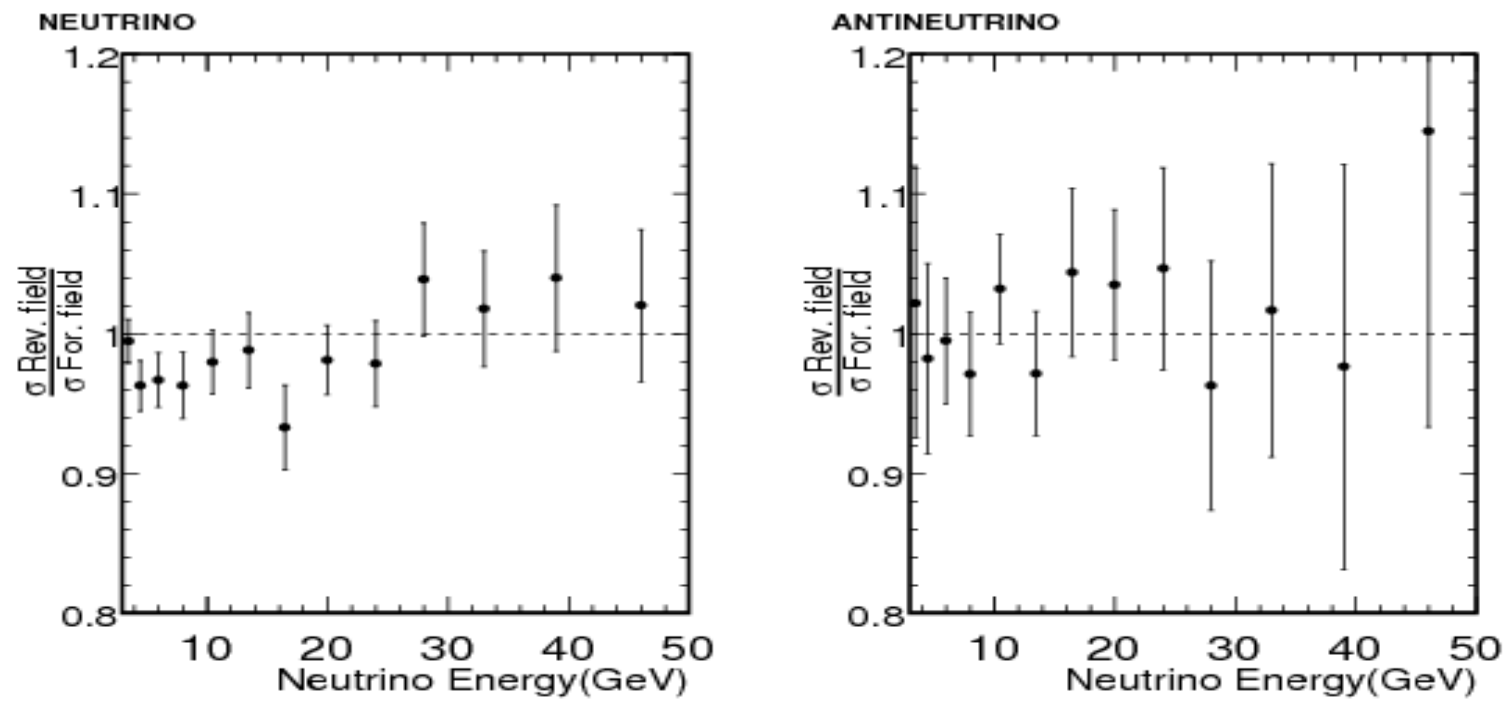

Figure 7.23: Ratio of reverse field to forward field extracted $\sigma / E$ for neutrinos(left) and antineutrinos(right). 

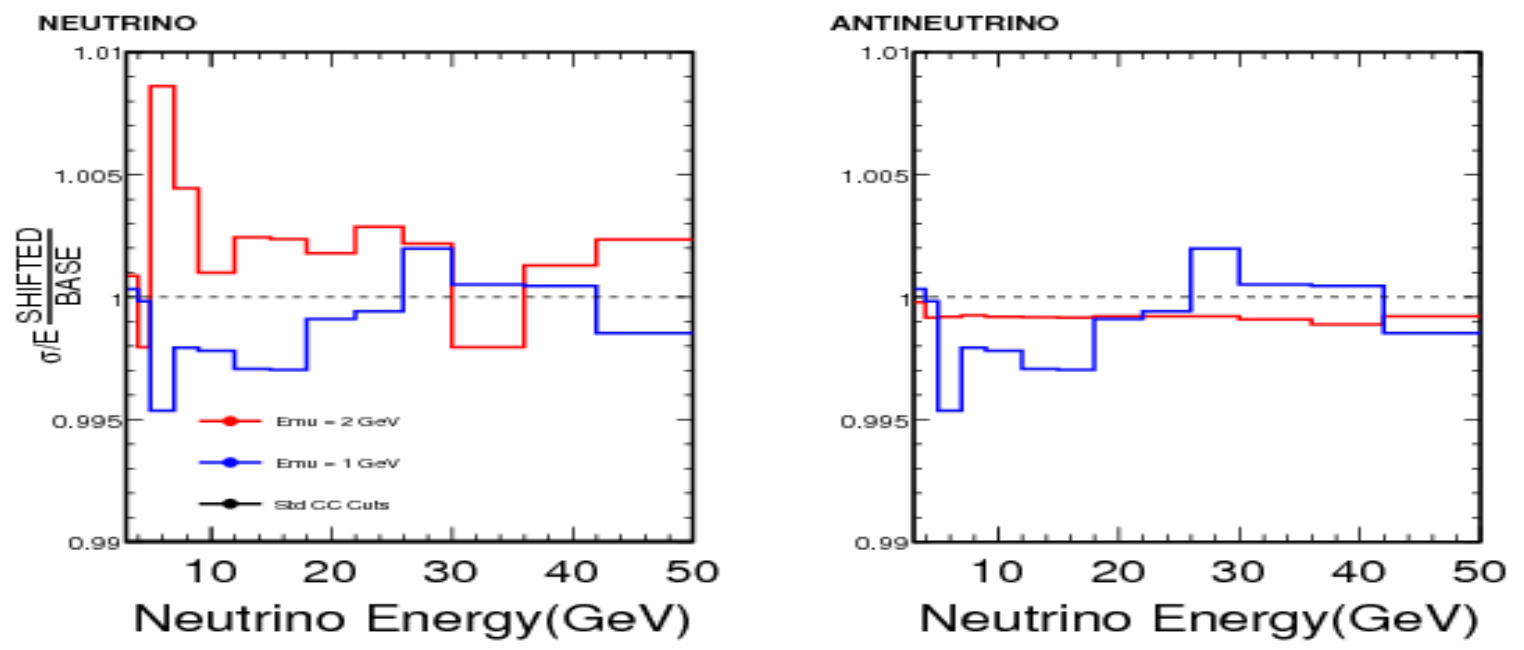

Figure 7.24: Effect of modifying the minimum $E_{\mu}$ cut from its standard value of $1.5 \mathrm{GeV}$ to $2 \mathrm{GeV}$ and $1 \mathrm{GeV}$ on the measured cross section.

ple. To estimate the uncertainty from this source we assume $+/-100 \%$ uncertainty on wrong-sign contamination. Fig. 7.25 shows the data and MC comparison of the antineutrino $\mathrm{CC}$ sample as a function of energy. This plot indicates a larger fractional wrong sign contamination in the data compared to the $\mathrm{MC}$ event sample. The resulting uncertainty on $\sigma / E$ is negligible at low energy $(<15 \mathrm{GeV})$ but is about $4 \%$ at high energy(42-50 GeV bin). Fig. 7.12 shows this uncertainty for the antineutrino $\sigma / E$.

We also used a different variable, called majority curvature (majC) to remove the wrong sign contamination from the sample and compared the results as a cross check of our sample contamination. The variable majC is calculated by breaking a track into small segments(where each segment has 8 scintillator strips) and performing a parabolic fit $a x^{2}+b x+c$ to each segment. The number of segments is counted with positive $(2 a>0)$ and negative $(2 a<0)$ curvature. If the number of positive curvature segments $(\mathrm{P})$ is larger than the number of negative curvature segments $(\mathrm{N})$, then the majC=P/N. If the opposite is true, then majC=-N/P. If the number of positive and negative segments are equal or the track has less than 8 scintillator strips, then the majC variable is assigned a value of 0 . Fig. 7.27 shows the majC distribution, where a majC $>0$ selection is applied to reject 

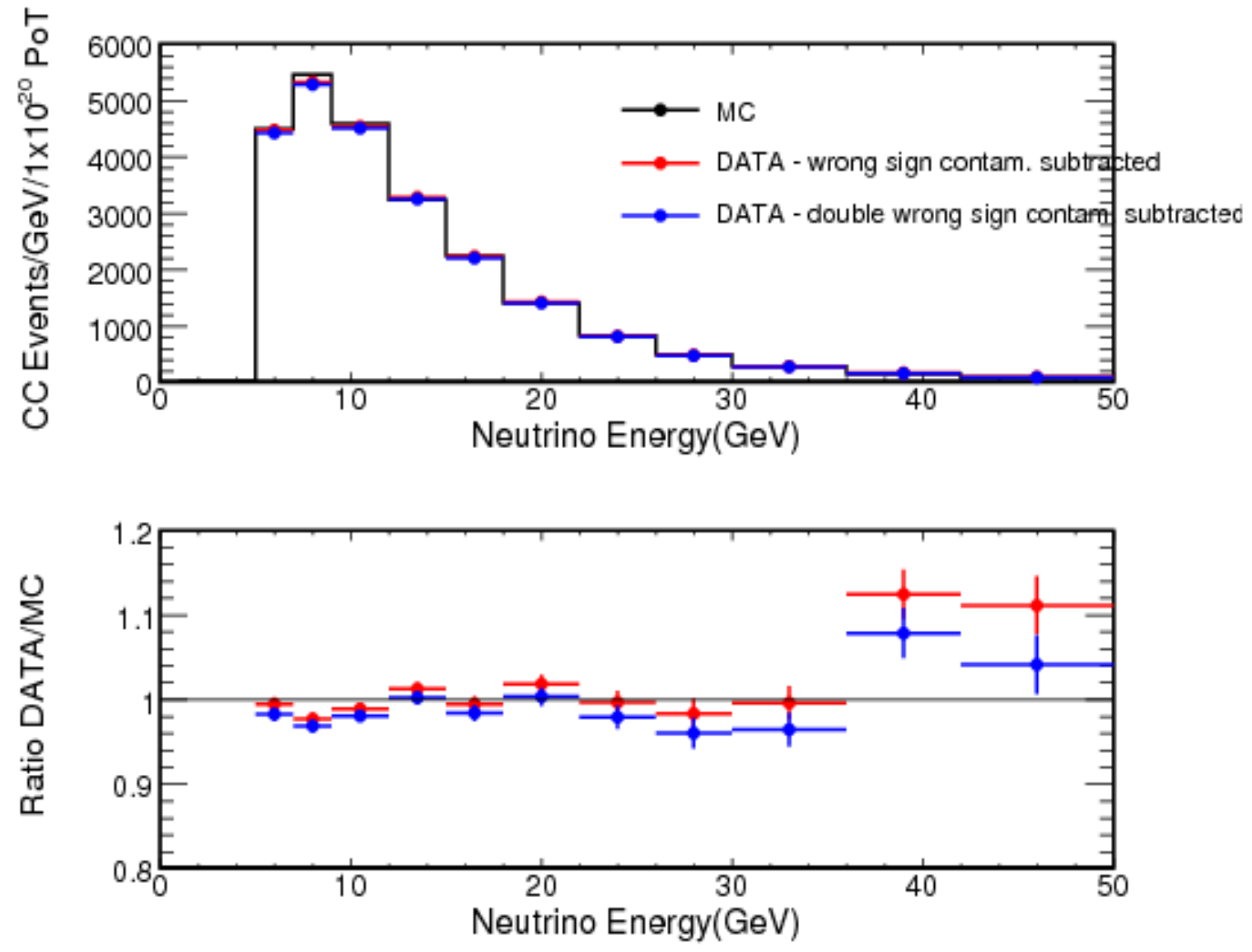

Figure 7.25: Data and MC comparison of MC comparison of the antineutrino CC sample as a function of energy. The top plot shows the distribution and the bottom plot shows the ratio of data to MC. Two cases are shown, one in which the standard MC contamination is subtracted from the data, the other in which double the MC contamination is subtracted. 


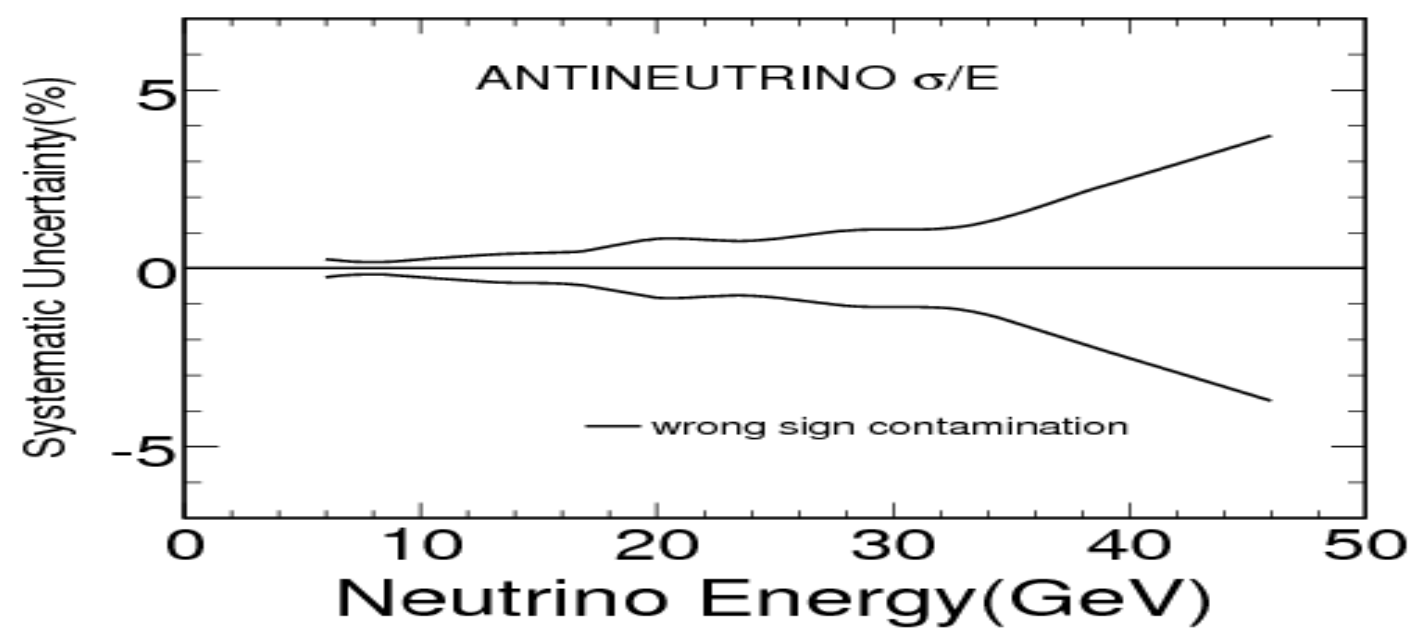

Figure 7.26: Systematic uncertainty from wrong sign contamination in the antineutrino extracted cross section.

wrong sign contamination. Fig. 7.28 shows a comparison of the fractional wrong sign contamination for the majC and relative angle selection criteria. The relative angle variable is slightly more efficient $(\sim 1 \%)$ than the majority curvature at high energy. The effect of varying this cut on the extracted cross section is very small $(<1 \%)$.

\subsubsection{Fiducial Volume}

The measured $\sigma / E$ was compared by separating events based on their interaction position in the detector. This study evaluated the effect of the detector geometry on the beginning position of the neutrino interactions. Fig. 7.29(a) and 7.30(a) show the fiducial volume divided into two segments in the transverse view: events beginning in the left versus right portion of the detector and events beginning in the lower versus upper section of the detector. The events beginning on the left portion of the detector have a greater influence from the coil hole compared to the ones beginning on the right. There are more neutrino interactions taking place in the lower half of the detector compared to the upper half because the neutrino beam bends downward by $\sim 3^{\circ}$. Fig. 7.29 (b) and 7.30(b) show that the extracted $\sigma / E$ for the different segments agreed to $1 \%$ within error bars and 


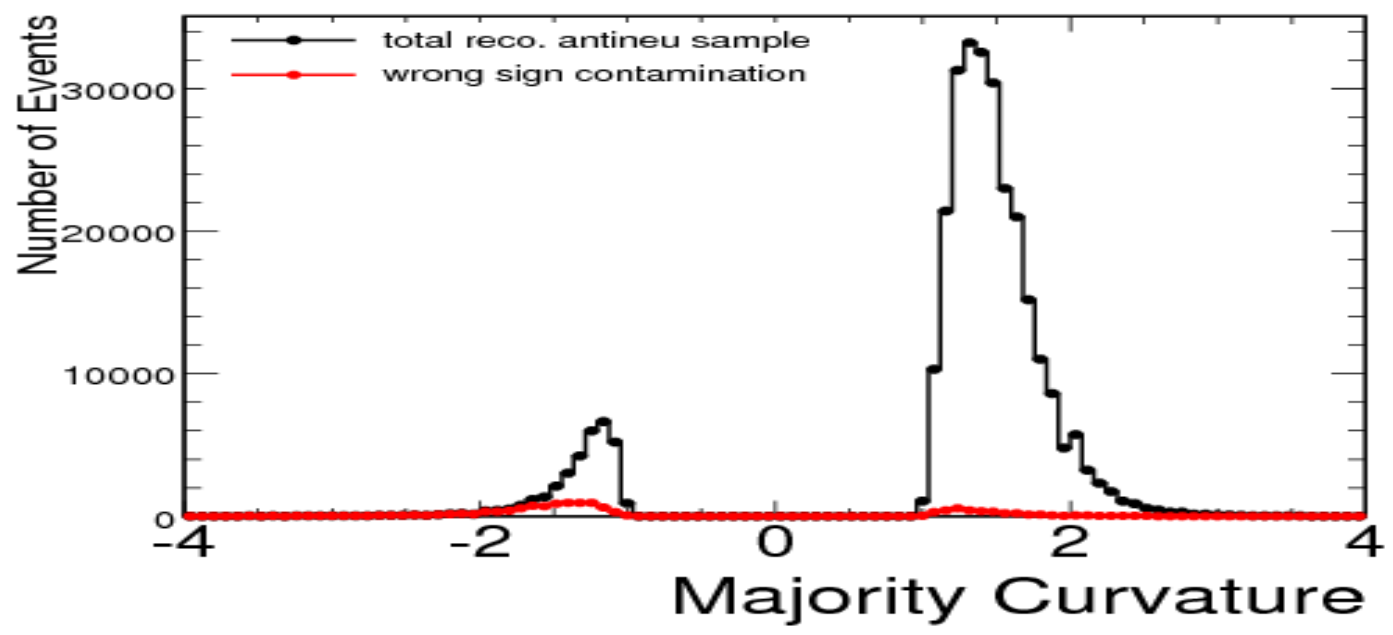

Figure 7.27: Distribution of majC for the total reconstructed antineutrino sample. The distribution for the wrong sign contamination is shown in red.

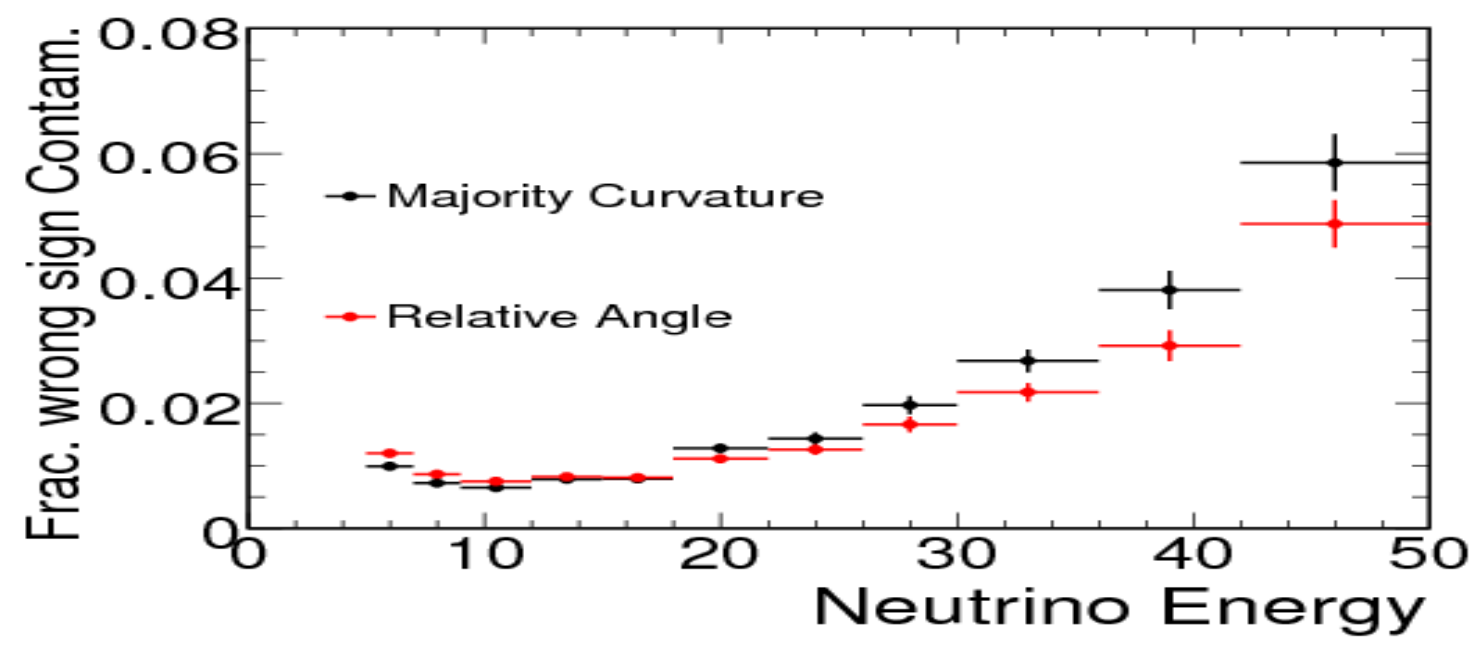

Figure 7.28: Fractional wrong sign contamination in the $\mathrm{CC}$ antineutrino sample by applying the relative angle(red) and majority curvature(black) selection criteria. 
no significant difference was observed. 


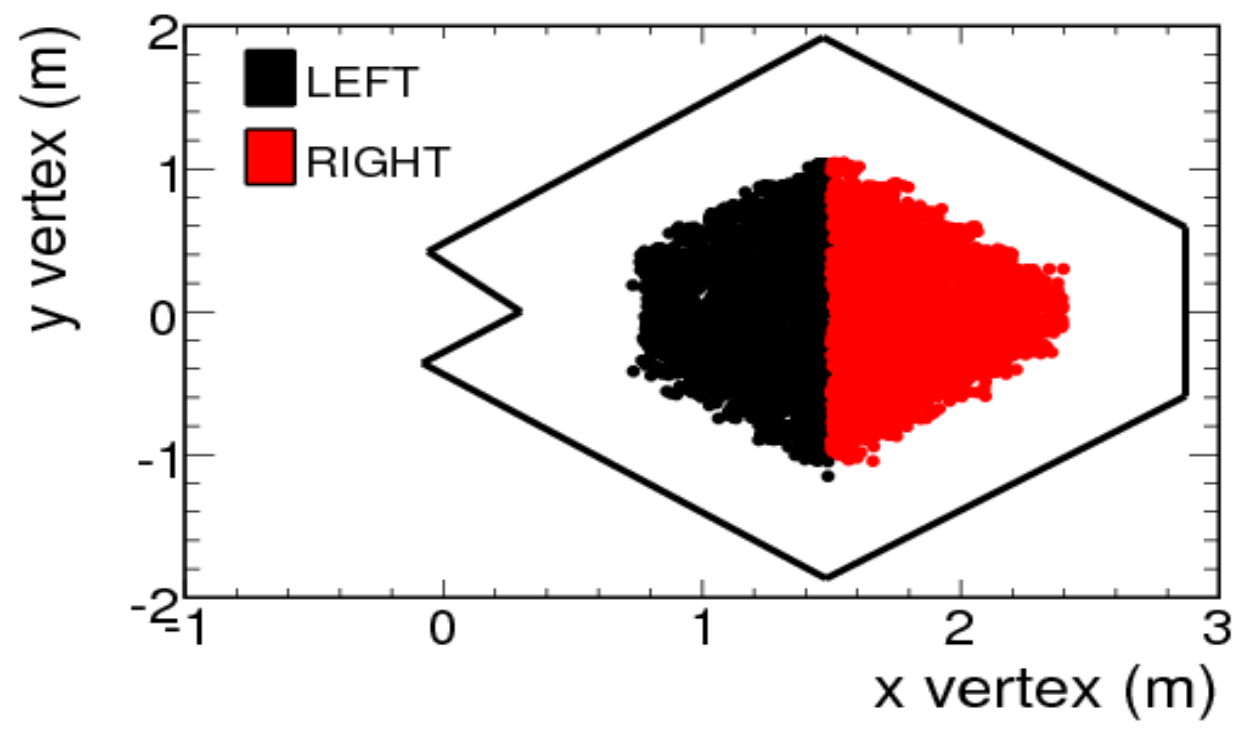

(a)
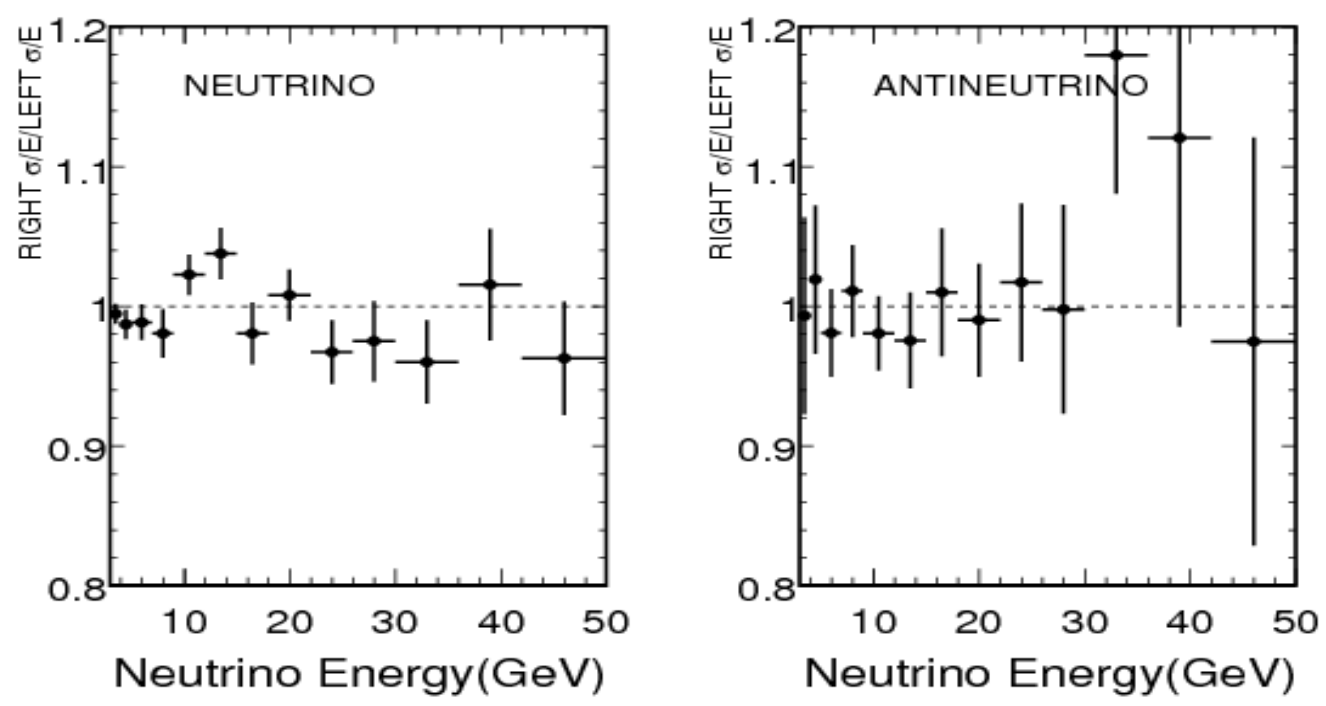

(b)

Figure 7.29: Fiducial volume divided into left and right sections in the transverse view using $\mathrm{x}$ vertex $>1.5 \mathrm{~m}(<1.5 \mathrm{~m})(\mathrm{a})$. Comparison of the measured $\sigma / E$ for neutrino and antineutrino between the two sections (b). 


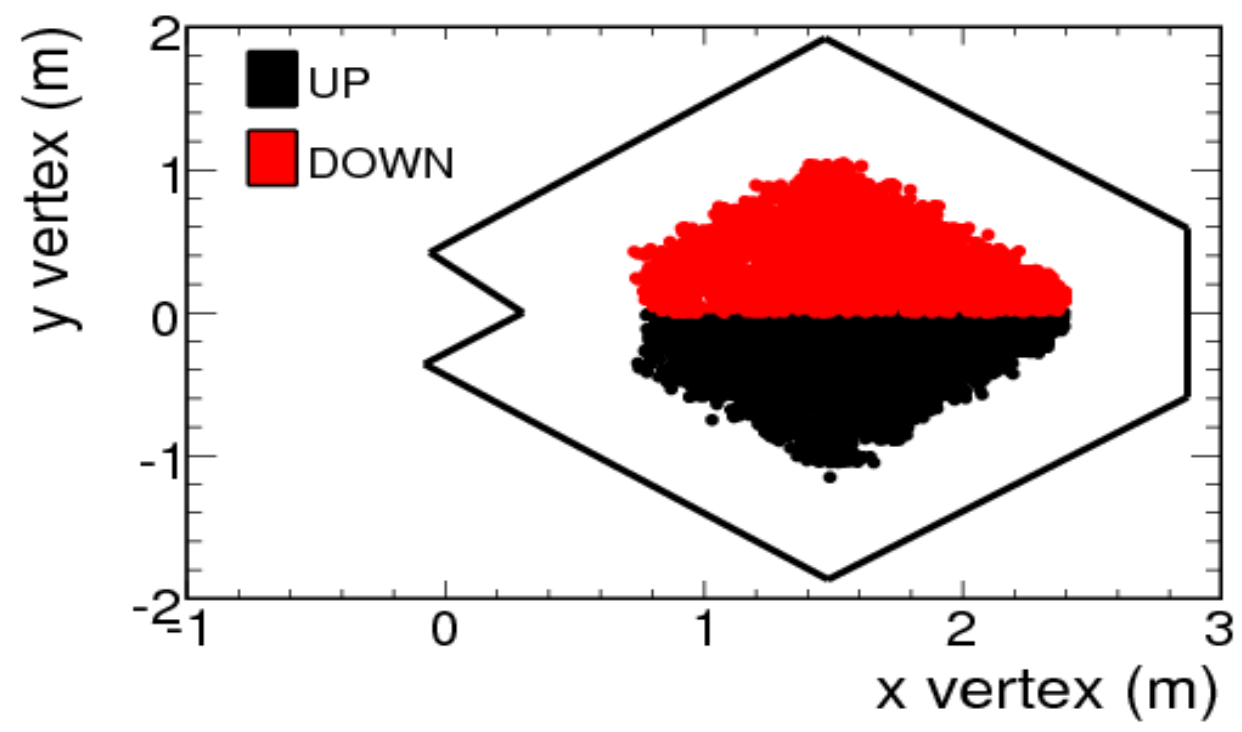

(a)
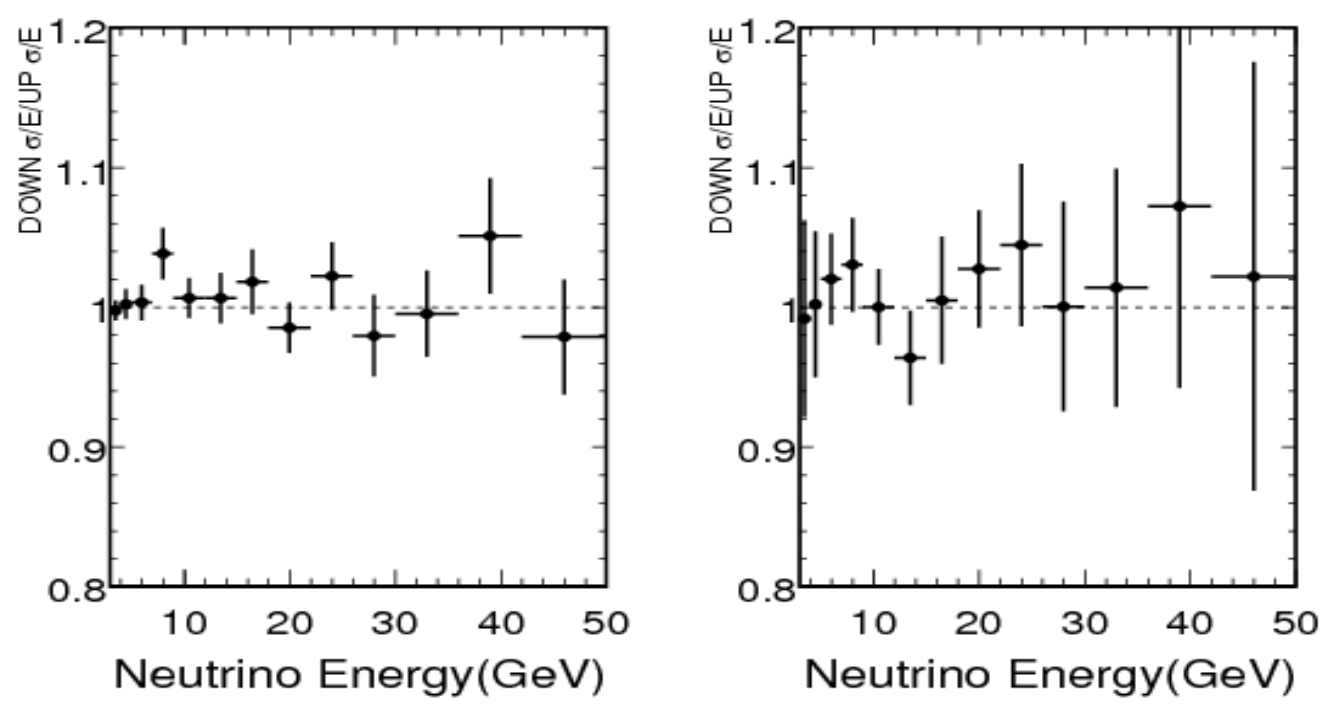

(b)

Figure 7.30: Fiducial volume divided into up and down sections in the transverse view using y vertex $>0 \mathrm{~m}(<0 \mathrm{~m})($ a). Comparison of the measured $\sigma / E$ for neutrino and antineutrino between the two sections (b). 


\subsection{RESULTS}

Fig. 8.1 shows the MINOS cross section results compared to measurements from other experiments. Neutrino and antineutrino $\sigma / E$ is independent of energy above $20 \mathrm{GeV}$. At lower energies the cross section shows deviations from the linear behavior; neutrino $\sigma / E$ decreases with energy whereas the antineutrino $\sigma / E$ increases with energy. This chapter presents a phenomenological description of the cross section results.

\section{1 $v_{\mu} N$ AND $\bar{v}_{\mu} N$ CC INCLUSIVE CROSS SECTION}

To explain the behavior of the measured cross section at high energy, we look at a simplified form of Eq. 1.31[53] based on the QPM

$$
\frac{d^{2} \sigma^{v(\bar{v}) N}}{d x d y}=\frac{G_{F}^{2} M E}{\pi}\left\{\left[\frac{F_{2}(x) \pm x F_{3}(x)}{2}\right]+\frac{F_{2}(x) \mp x F_{3}(x)}{2}(1-y)^{2}\right\},
$$

where, $F_{2}=x[u(x)+d(x)+\bar{u}(x)+\bar{d}(x)], x F_{3}=x[u(x)+d(x)-\bar{u}(x)-d(x)]$. In this model the

PDFs are assumed to be independent of $Q^{2}$ and depend only on x. Integrating 8.1 over x and y from 0 to 1 gives the total cross section

$$
\begin{aligned}
& \sigma(v N)=\frac{G_{F}^{2} M E}{\pi}\left(P+\frac{1}{3} \bar{P}\right), \\
& \sigma(\bar{v} N)=\frac{G_{F}^{2} M E}{\pi}\left(\bar{P}+\frac{1}{3} P\right),
\end{aligned}
$$

where, $P=\int x[u(x)+d(x)] d x, \bar{P}=\int x[\bar{u}(x)+\bar{d}(x)] d x$. The term (1/3)P in $\sigma(\bar{v} N)$ can be explained by momentum conservation. The axial vector nature of the weak interaction projects only the left 


\begin{tabular}{|c|c|c|c|c|c|c|c|c|c|c|}
\hline$E_{V}(\mathrm{GeV})$ & 5.5 & 7.5 & 9.5 & 15.5 & 20.5 & 25.5 & 30.5 & 35.5 & 40.5 & 50.5 \\
\hline \hline$<Q^{2}>\left(\mathrm{GeV}^{2}\right)$ & 1.44 & 1.92 & 2.39 & 3.72 & 4.82 & 5.92 & 7.04 & 7.91 & 9.20 & 11.11 \\
\hline
\end{tabular}

Table 8.1: Average $Q^{2}$ for a given energy value calculated from MINOS generated sample.

handed particles and right handed antiparticles as shown in Fig. 8.2. For the $v$ quark scattering, since both the neutrino and quark are left handed, the total angular momentum is $J=0$. The $\bar{v}$ quark scattering on the other hand has $J=1$ and $J_{Z}=1$ for the initial and final state. The number of possible final substates are $2 J+1\left(J_{Z}=0, \pm 1\right)$, but out of these three states only the $J_{Z}=1$ state is allowed which leads to a suppression factor of 3 . The factor of $(1 / 3) \bar{P}$ in $\sigma(v N)$ can be similarly explained by looking at neutrino(antineutrino) antiquark scattering.

A prediction for the energy dependence of the cross section can be calculated using Eq. 8.2 where $P$ and $\bar{P}$ have been computed from GRV 98 PDFs[39]. The $Q^{2}$ dependence of the PDFs is taken into account using the average $Q^{2}$ from a distribution of the MINOS generated sample. Table 8.1 shows the average $Q^{2}$ for each energy value. To ensure that the calculation is within the pure deep inelastic scattering regime, a minimum $W$ threshold has been applied to reject resonance contribution. Two cases have been taken into account, one in which a minimum $W$ threshold of $1 \mathrm{GeV}$ is applied and another in which a minimum $W$ of $2 \mathrm{GeV}$ is applied. We have taken into account up, down and strange quark PDF distributions, although Eq.8.2 assumes up and down quark only.

Fig. 8.3 shows the energy dependence of the terms $P+\frac{1}{3} \bar{P}$ and $\bar{P}+\frac{1}{3} P$. The scaling of cross section (neutrino nucleon scattering can be approximated to weak elastic scattering from pointlike partons) with increasing energy is clearly demonstrated by this plot above $20 \mathrm{GeV}$. For such interactions the total cross section is proportional to the center of mass energy (Eq. 1.11), $s \sim 2 M E$, i.e. the cross section is linearly dependent on energy.

The $P+\frac{1}{3} \bar{P}$ and $\bar{P}+\frac{1}{3} P$ terms are non-linear at low energy due to the $Q^{2}$ dependence of the PDFs. Fig. 8.4 shows the energy dependence of the terms $P$ and $\bar{P}$ to better understand this behavior. $P$ has contributions from valence quarks and sea quarks whereas $\bar{P}$ has contributions from sea quarks only. For a given $Q^{2}$ value, the distribution of the PDFs as a function of $\mathrm{x}$ is very 
different for the valence quarks and sea quarks. The valence quark distribution peaks at moderate $\mathrm{x}$ and goes to 0 at $\mathrm{x} \rightarrow 0$. If the nucleon consisted of only three valence quarks then these PDFs would have peaked around $x=1 / 3$ but it peaks at a smaller $x$ value. This is because the nucleon momentum is shared between valence quarks, gluons and quark-antiquark pairs. The sea quark distribution peaks at low $\mathrm{x}$ and decreases to 0 around $\mathrm{x} \sim 0.5$. Gluon splitting at low $\mathrm{x}$ creates a large number of sea quark-antiquark pairs that dominate the PDFs at smaller value of $\mathrm{x}$.

The $Q^{2}$ dependence of the PDFs vary in the low and high $\mathrm{x}$ regions. At low $\mathrm{x}$, both the sea quark and valence quark PDFs increase with $Q^{2}$. This is caused by the increase in the resolving power of the neutrino probe. At larger x values, the valence PDFs decrease with $Q^{2}$. This happens because as the resolution increases it becomes less probable to see partons with large momentum.

Fig. 8.4 shows that the $\bar{P}$ term increases with $Q^{2}$ as expected. The $P$ term has a much stronger dependence on energy compared to the $\bar{P}$ term. If we look at the $\mathrm{W}<1 \mathrm{GeV}$ case, we see a decrease in the $P$ term with increasing energy (or $Q^{2}$ ). This is caused by the dominance of the decreasing valence PDFs with $Q^{2}$ at large $\mathrm{x}$ values. Applying a $W$ cut can significantly modify the energy dependence of the $P$ term. At low $Q^{2}$, the PDFs are a function of $Q^{2}$ and $\mathrm{x}$. To obtain $P$ we integrate over $\mathrm{x}$ from 0 to 1 , but applying a threshold $\mathrm{W}$ cut reduces the upper limit of the integration from 1 to $Q^{2} /\left(W_{\min }^{2}-M_{\text {nucleon }}^{2}+Q^{2}\right)$. A lower $W_{\text {min }}$ would have a larger upper integration limit on x at low $Q^{2}$. As $Q^{2}$ increases, the integration limit approaches 1 and $P$ becomes independent of the $\mathrm{W}$ cut. For a W cut of $2 \mathrm{GeV}$, we see that the $P$ term increases with $Q^{2}$ which is caused by the rejection of the valence quark PDF distributions at large $\mathrm{x}$ values. The valence quark PDFs have a significant contribution in the large $\mathrm{x}$ region. The $W$ cut has very little effect on the antiquark distribution. These PDFs are very small above $\mathrm{x}$ of 0.5 , hence reducing the upper limit of $\mathrm{x}$ integration doesn't modify the $\bar{P}$ term.

The energy dependence of the $P+\frac{1}{3} \bar{P}$ term is dominated by the $P$ term while the $\bar{P}+\frac{1}{3} P$ shape is affected by both the $P$ and $\bar{P}$ term. Further $Q^{2}$ dependence is introduced by elastic and resonance interactions that contribute in the low energy region.

The physics of QEL and RES interactions can be explained by replacing structure functions with form factors (described in Eq. 1.15) which can be interpreted as the probability for a nucleon to stay "intact" in a neutrino nucleon interaction. This becomes less probable as the $Q^{2}$ of the interaction increases which leads to a decrease in the cross section for these processes with 
increasing energy. The QEL and RES cross sections for antineutrinos are smaller than the neutrino cross sections at low energies because of the overlap of the axial vector and vector form factors. At higher energies, the neutrino and antineutrino QEL and RES cross sections approach each other and become constant due to the rapid decrease of the form factors with $Q^{2}$.

Fig. 8.1 shows that the MINOS neutrino and antineutrino measured cross sections approach scaling behavior around $20 \mathrm{GeV}$ and hence are completely dominated by deep inelastic interactions above this energy. At lower energies, the energy dependence of the cross section arises from the relative contribution of elastic and inelastic cross sections, which are different for neutrino and antineutrino. The neutrino cross section is dominated by the decreasing elastic cross section. On the other hand, the antineutrino cross section is dominated by the increasing inelastic cross section which indicates that the antiquark degrees of freedom are increasing with energy. The antiquark PDFs show a stronger dependence on energy than that calculated by using GRV 98 PDFs( Fig. 8.4 ) which could be caused by the fact that these PDFs are leading order.

The MINOS neutrino cross section shows good agreement with previous measurements from CRS[12], SKAT[16] and GGM-PS[28], but these experiments have significantly larger uncertainties. The BNL-7ft[21] and IHEP-JINR[31] report cross section results in the $0.4-30 \mathrm{GeV}$ energy range with comparable precision to that of MINOS. However, in IHEP-JINR experiments the flux was determined using a quasi-elastic cross section which was not well known ${ }^{1}$ and the BNL-7ft result quotes only the statistical error. MINOS is in good agreement with the recent NOMAD results at high energies $(>7 \mathrm{GeV})$ but has a different shape at lower energies although the results overlap within error bars. The largest uncertainty in MINOS comes from knowledge of the absolute muon and hadronic energy scales whereas in NOMAD the flux determination dominates the uncertainty. MINOS measurements have the smallest uncertainty in the 10-30 GeV energy range. The MINOS antineutrino cross section results are in good agreement with the sparse data available at lower energies and has much smaller uncertainties in the 10-30 GeV region.

Fig. 8.5 shows the MINOS measured cross section compared to the predicted cross section by the NEUGEN model. The measured cross section agrees with the predicted one within error bars although the predicted asymptotic antineutrino cross section above $20 \mathrm{GeV}$ is lower than the

\footnotetext{
${ }^{1}$ The assumed uncertainty was $5 \%$ on the $M_{a}(Q E L)$ parameter. Based on recent experimental observations, a more realistic uncertainty on the $M_{a}(Q E L)$ is $15 \%$.
} 
measured value.

\subsection{RATIO OF $\bar{v}_{\mu} N$ CC TO $v_{\mu} N$ CC INCLUSIVE CROSS SECTION}

Fig. 8.6 shows the measured ratio of the $\bar{v}_{\mu} N$ CC to $v_{\mu} N$ CC inclusive cross section compared to the QPM predicted value

$$
\frac{\sigma(\bar{v})}{\sigma(v)}=\frac{1+3 \bar{Q} / Q}{3+\bar{Q} / Q}
$$

The high energy behavior is explained well by the simple model which shows that the antineutrino cross section is about half of the neutrino cross section at high energies.

Fig. 8.6 shows that the measured ratio of the $\bar{v}_{\mu} N \mathrm{CC}$ to $v_{\mu} N \mathrm{CC}$ inclusive cross section becomes independent of energy above $30 \mathrm{GeV}$ as predicted by the naive QPM. The average ratio in the $30-50 \mathrm{GeV}$ range $(0.489 \pm 0.012)$ agrees with the value measured from other experiments $(0.504 \pm 0.003)$. The MINOS average measured value of $\sigma(\bar{v}) / \sigma(v)$ between $30-50 \mathrm{GeV}$ can be used to determine the ratio $\bar{P} / P$ which is $0.186 \pm 0.005$. This indicates that the antiquarks carry about $19 \%$ of the quark momentum. Fig. 8.7 shows the $\bar{P} / P$ ratio as a function of energy calculated from QPM. The toy model discussed here predicts the asymptotic value of $\bar{P} / P$ to be about $19 \%$ which depends on the GRV 98 PDF set.

In the low energy region, the measured $\sigma(\bar{v}) / \sigma(v)$ ratio increases with energy which indicates an increase in the antineutrino cross section which is caused by the increase in anti-quark PDFs with $Q^{2}$. The QPM predicted curves shown in Fig. 8.6 have different behavior in the low energy region for the two $\mathrm{W}$ cuts. The $\sigma(\bar{v} N)$ term is independent of energy for $\mathrm{W}>1 \mathrm{GeV}($ Fig. 8.3), hence the energy dependence of the ratio term is mostly from the QCD evolution of the $\sigma(v N)$ term. For $\mathrm{W}>2 \mathrm{GeV}$, the energy dependence of the ratio is contributed by both the $\sigma(\bar{v} N)$ and $\sigma(v N)$ term.

Fig. 8.8 shows the measured ratio of the $\bar{v}_{\mu} N$ CC to $v_{\mu} N$ CC cross section compared to the predicted value from the NEUGEN cross section model. There is good agreement with the predicted value within error bars. 


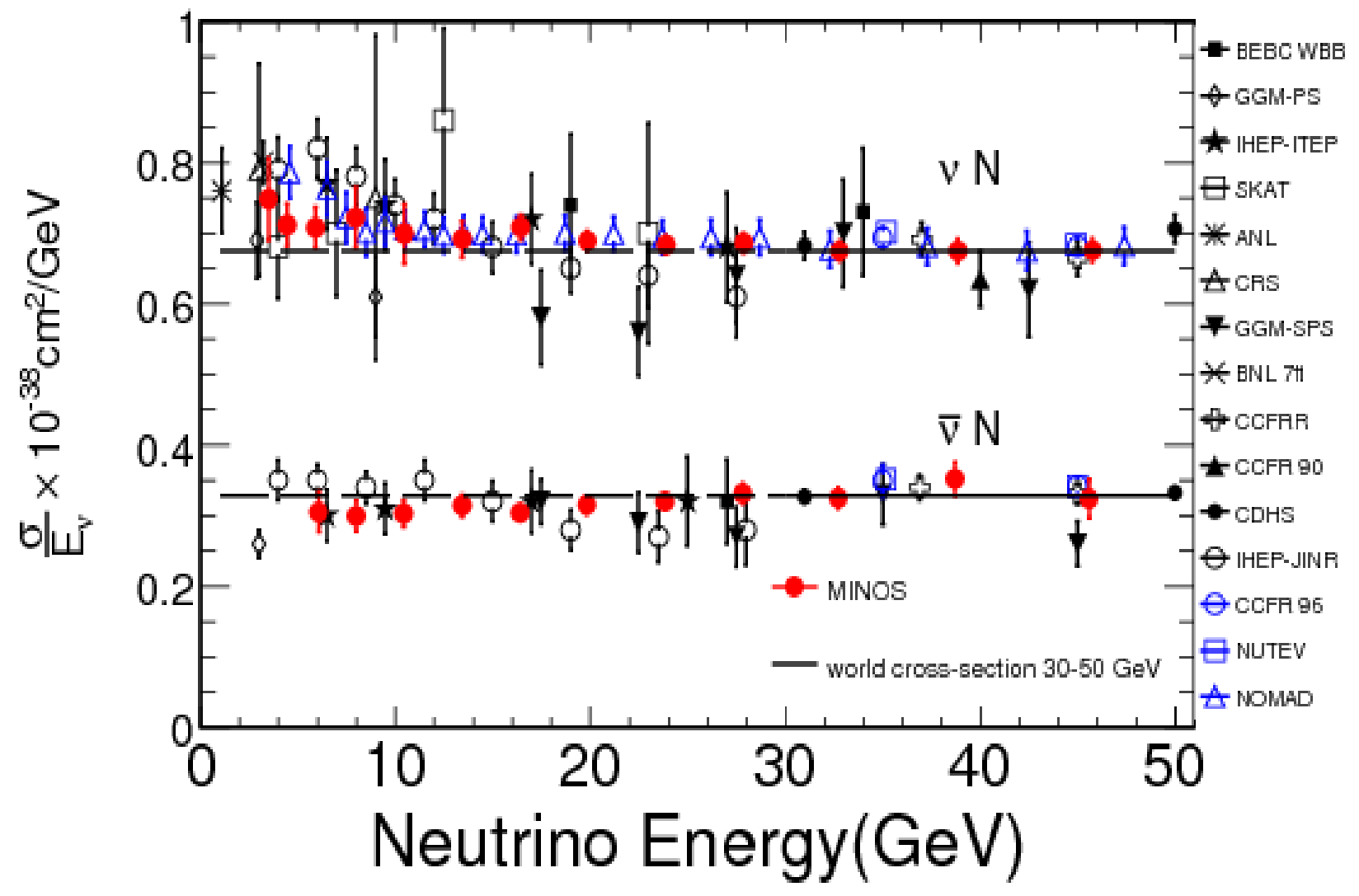

Figure 8.1: MINOS neutrino and antineutrino charged-current inclusive cross section compared with other experimental[18,31, 26, 21, 12, 16, 30, 25, 28, 14, 23, 46, 17, 55, 20, 11, 27] results. The error bars show the statistical, systematic and normalization uncertainties added in quadrature. The solid black line shows the average world cross section in the $30-50 \mathrm{GeV}$ region for the neutrino $\left(0.675 \times 10^{-38} \mathrm{~cm}^{2} / \mathrm{GeV}\right)$ and the antineutrino $\left(0.329 \times 10^{-38} \mathrm{~cm}^{2} / \mathrm{GeV}\right)$ cross section respectively. The dashed line shows these high energy values extrapolated to lower energies. 

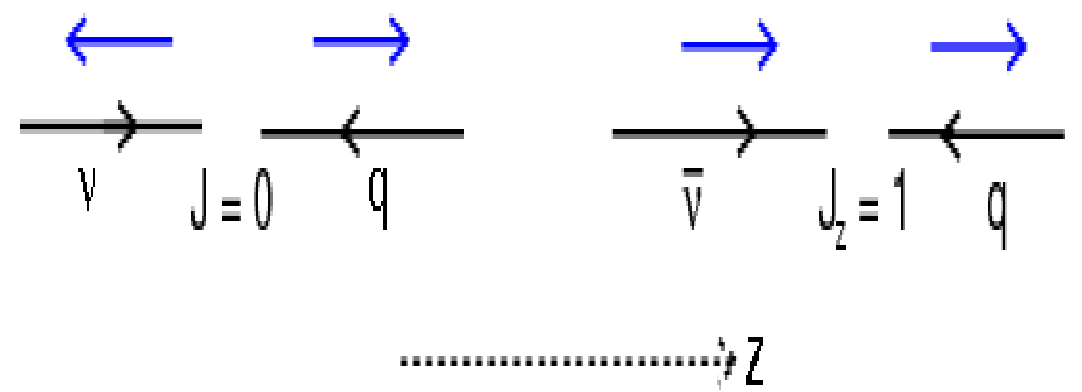

Figure 8.2: Helicity of neutrinos(antineutrinos) and quarks in a weak interaction. The blue arrows show the direction of the spin and the black arrows show the direction of the momentum.

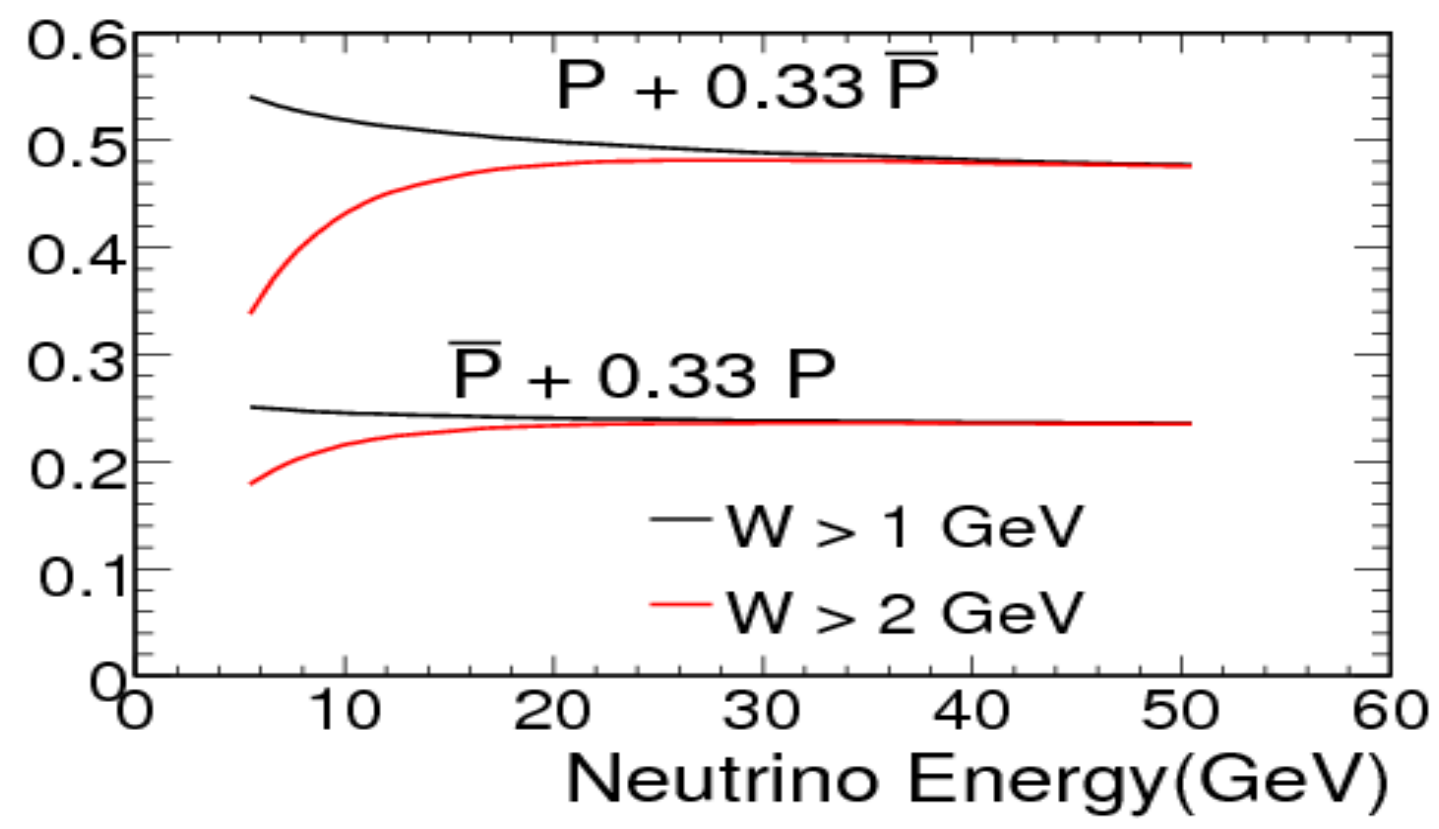

Figure 8.3: Energy dependence of the terms $\left(P+\frac{1}{3} \bar{P}\right)$ and $\left(\bar{P}+\frac{1}{3} P\right)$. The black line shows the value of this quantity when a $\mathrm{W}$ cut of $1 \mathrm{GeV}$ is applied and the red line shows the quantity for a $\mathrm{W}$ cut of $2 \mathrm{GeV}$. 


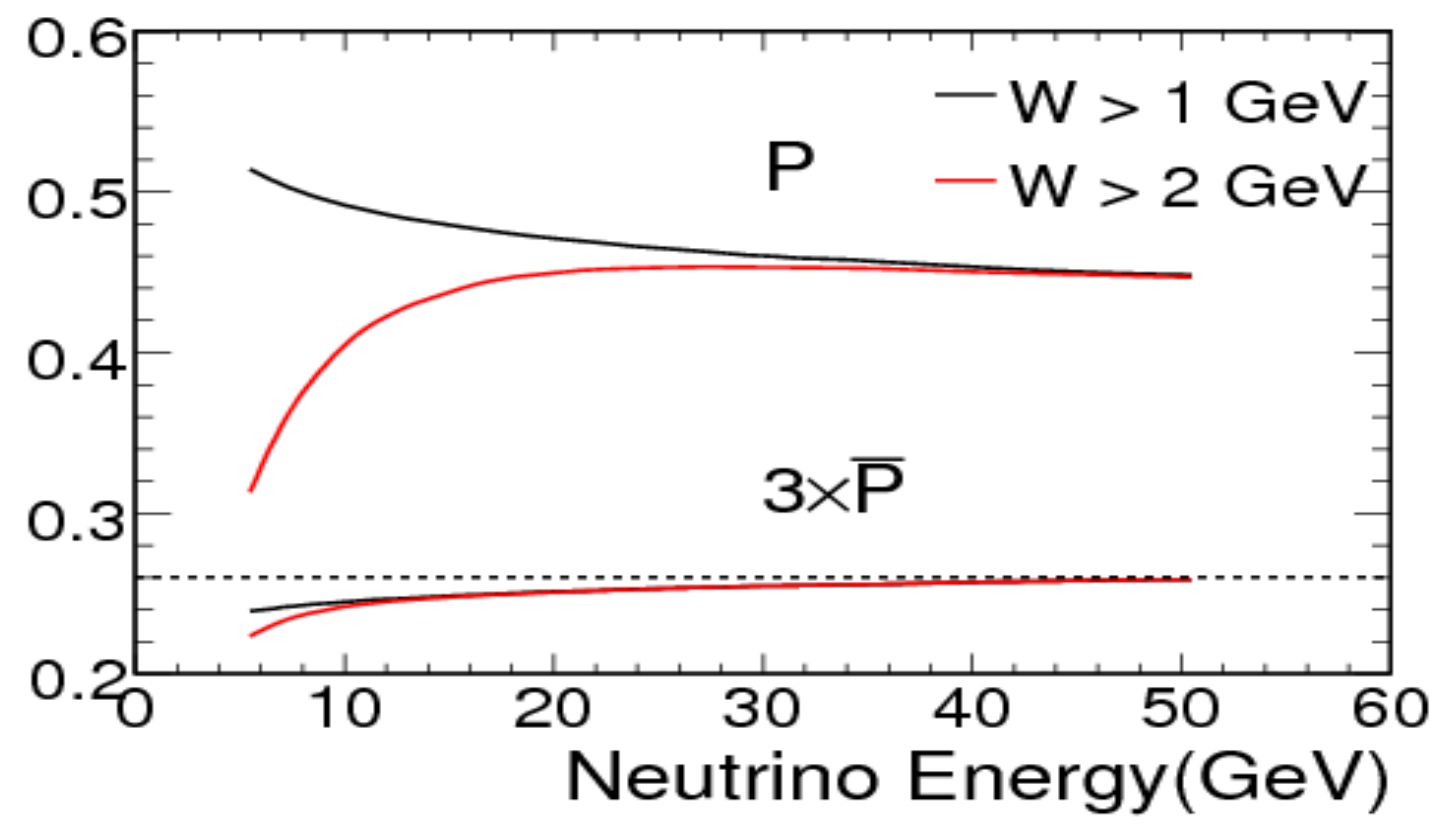

Figure 8.4: Energy dependence of the terms $P$ and $\bar{P}$. The black line shows the value of this quantity when a $\mathrm{W}$ cut of $1 \mathrm{GeV}$ is applied and the red line shows the quantity for a $\mathrm{W}$ cut of 2 $\mathrm{GeV}$. 


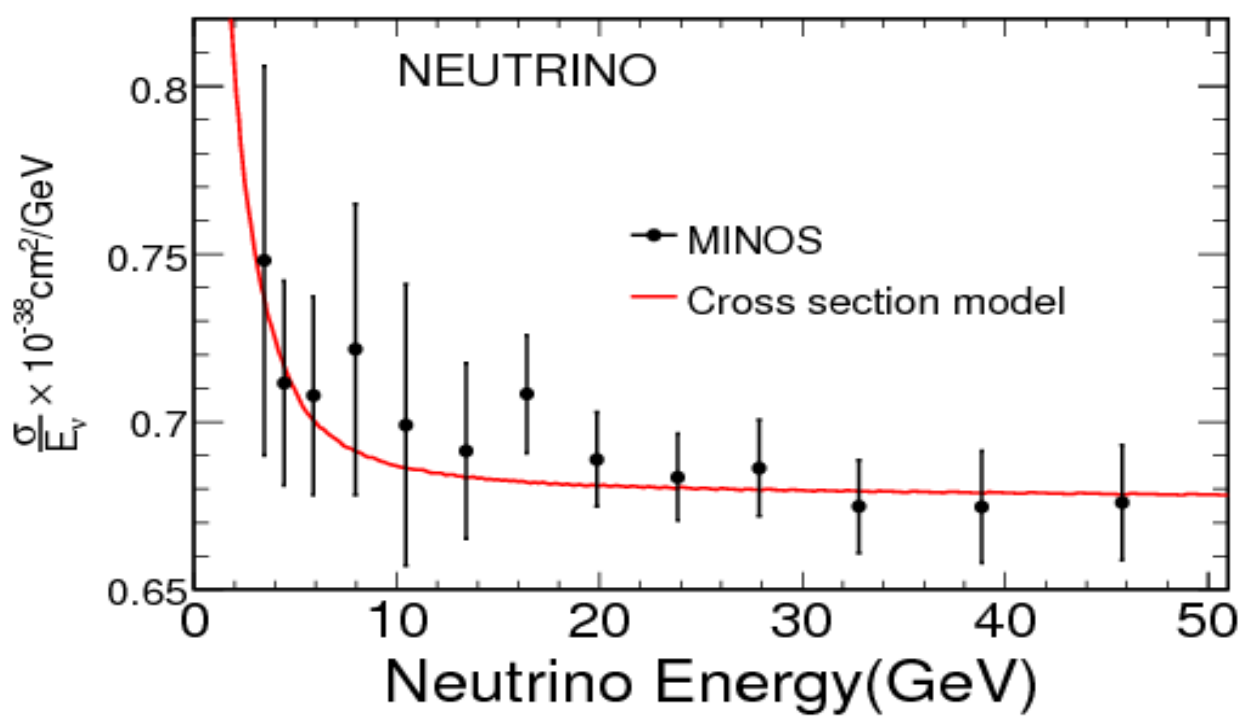

(a)

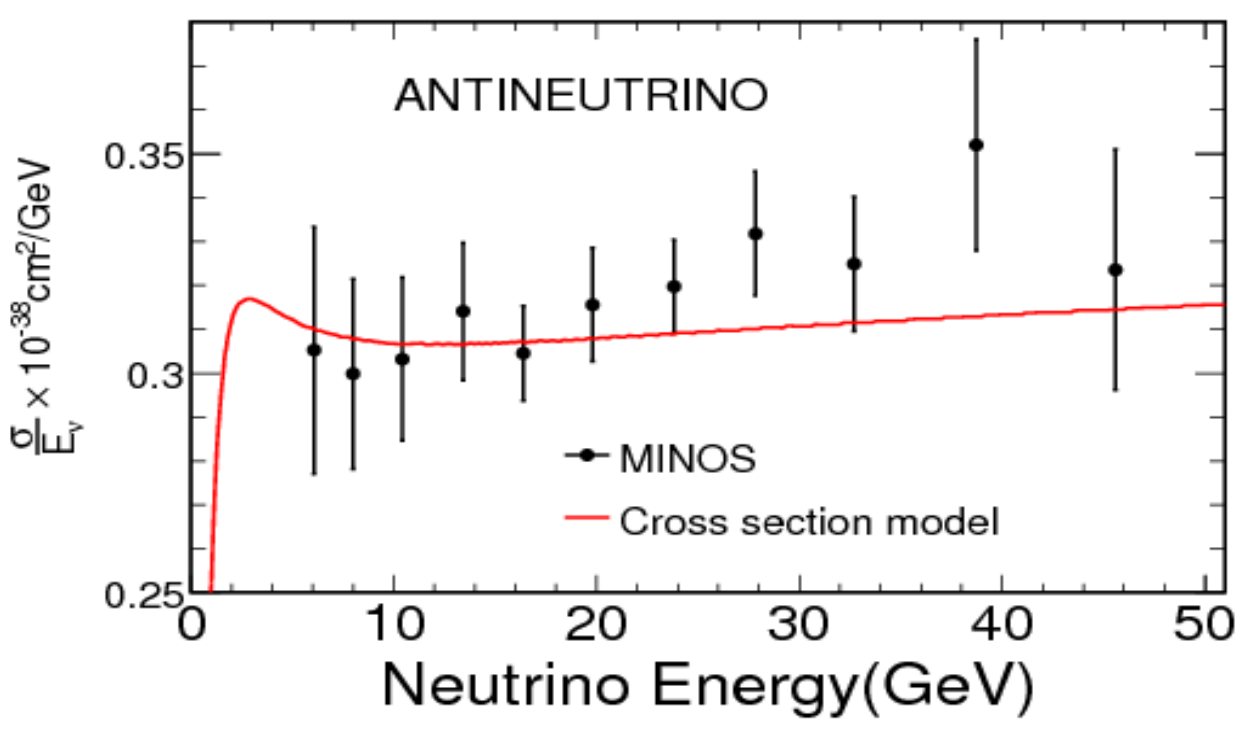

(b)

Figure 8.5: MINOS measured cross section compared to the cross section predicted by the NEUGEN cross section model for neutrinos(a) and antineutrinos(b). 


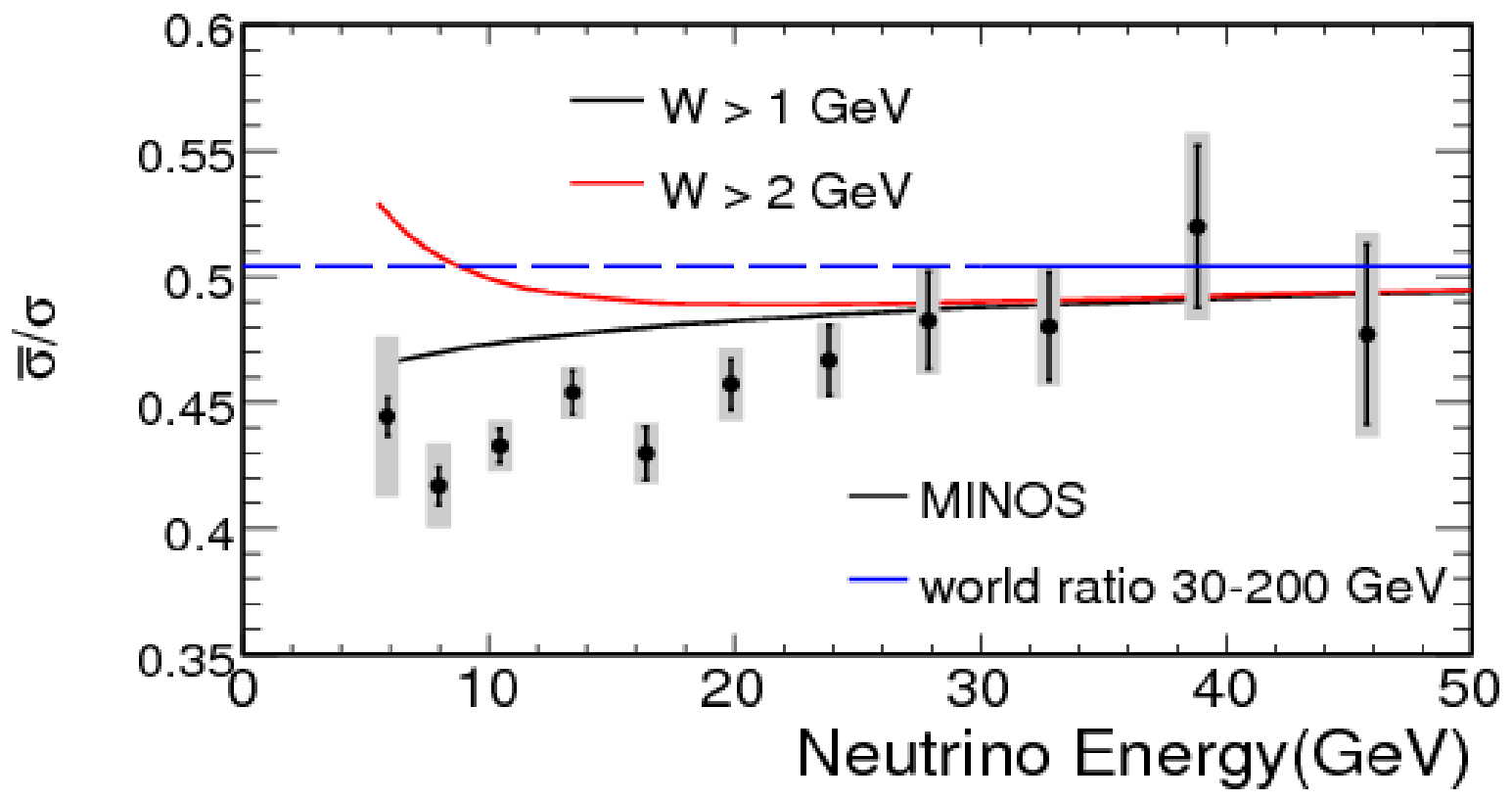

Figure 8.6: Ratio of antineutrino-nucleon to neutrino-nucleon cross section as a function of energy. The black error bars show the statistical uncertainty and the shaded boxes show the statistical and systematic uncertainty added in quadrature. The solid blue line shows the value of this ratio from other experiments between $30-200 \mathrm{GeV}$ at $0.504 \pm 0.003$. Ratio of $P+\frac{1}{3} \bar{P}$ to $\bar{P}+\frac{1}{3} P$ is plotted as a function of energy. The black line shows this term when a $\mathrm{W}$ cut of $1 \mathrm{GeV}$ is applied and the red line shows this term when a $\mathrm{W}$ cut of $2 \mathrm{GeV}$ is applied. 


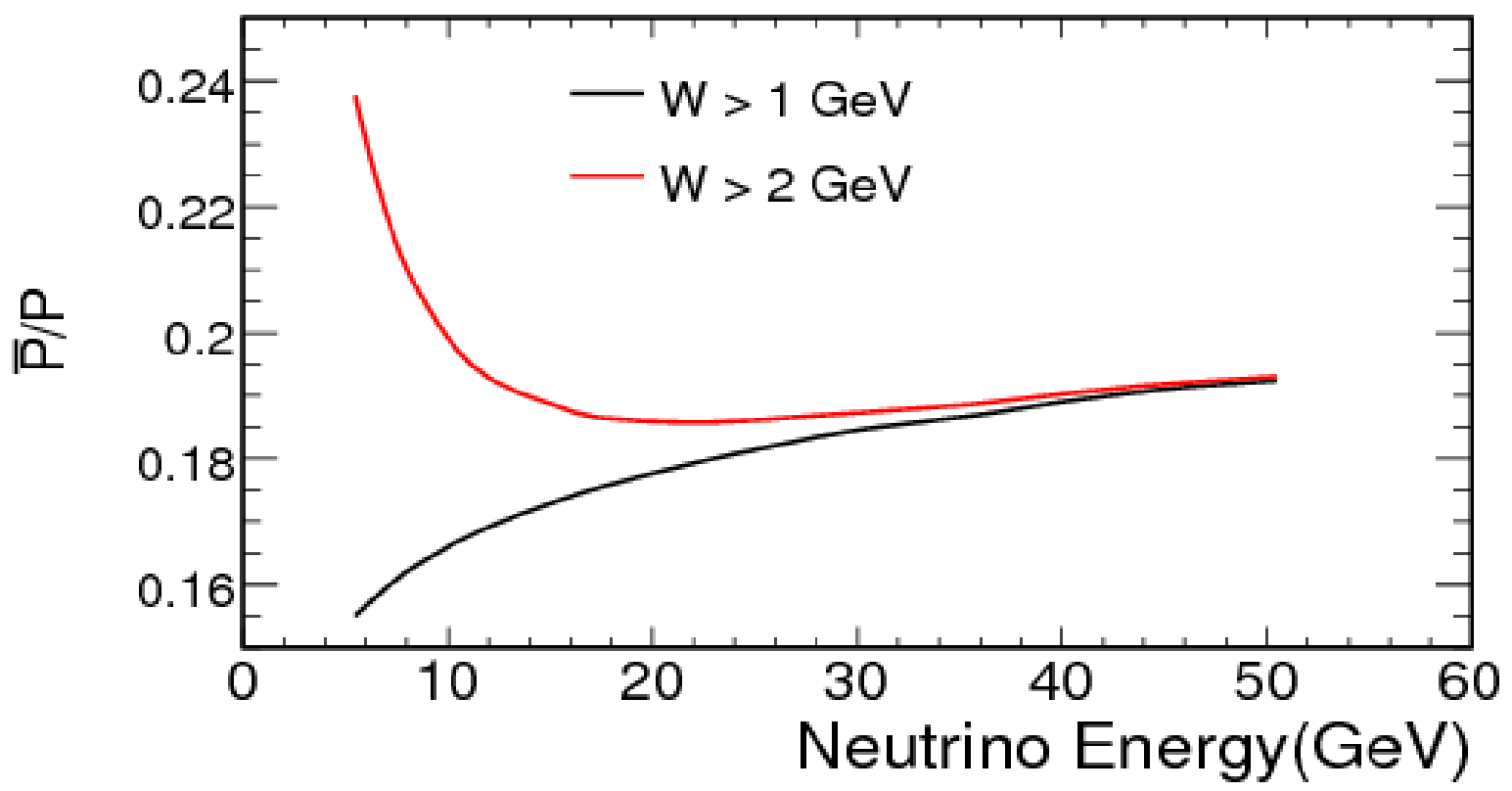

Figure 8.7: Ratio of $\bar{P} / P$ is plotted as a function of neutrino energy. The black line shows the value of this quantity when a $\mathrm{W}$ cut of $1 \mathrm{GeV}$ is used and the red line shows the value of this quantity when a $\mathrm{W}$ cut of $2 \mathrm{GeV}$ is used. 


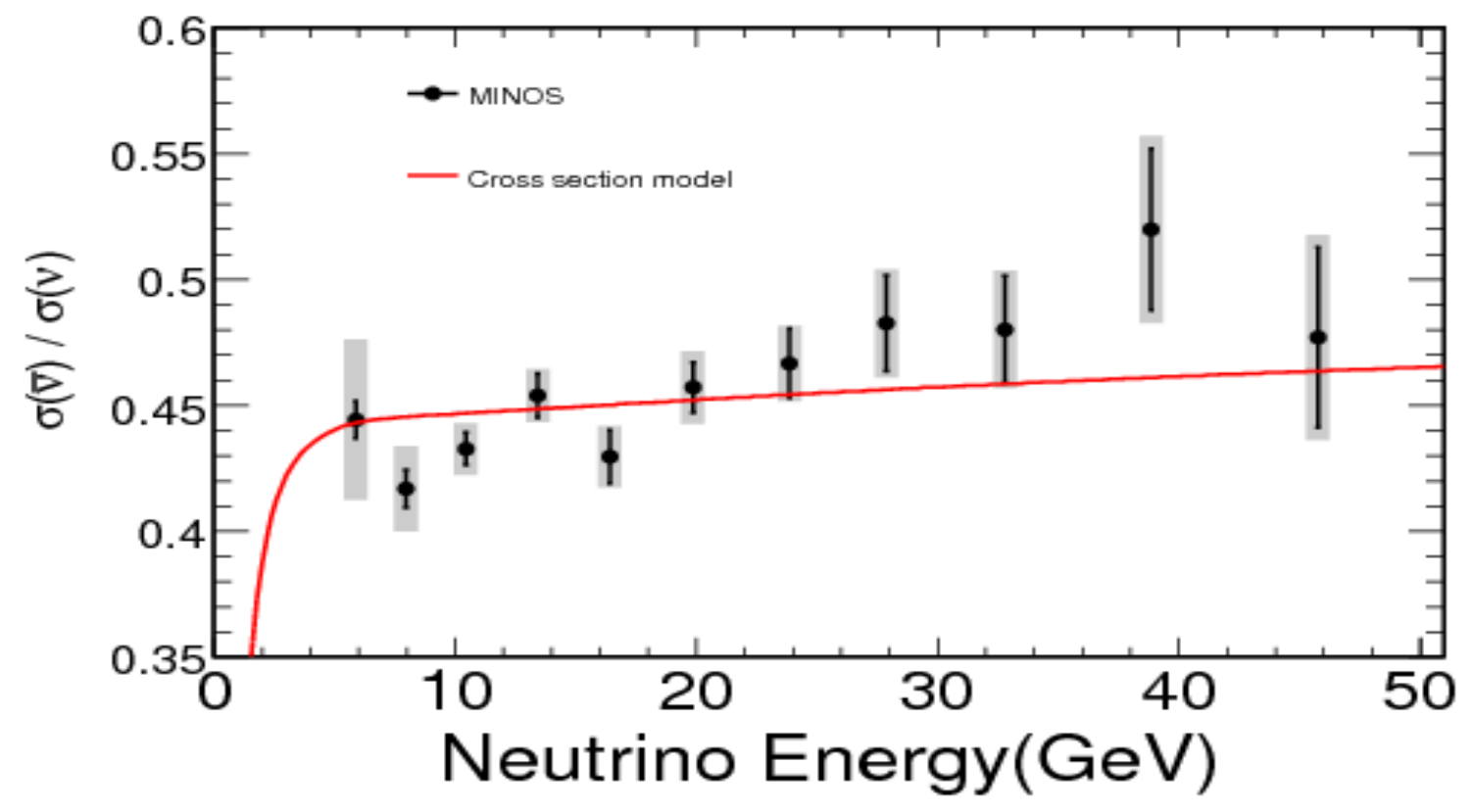

Figure 8.8: Ratio of antineutrino to neutrino cross section as a function of energy. The red line shows this ratio calculated from the cross section model. 
In this thesis I have presented $v_{\mu} N \mathrm{CC}\left(\bar{v}_{\mu} N \mathrm{CC}\right)$ inclusive cross sections and their ratio in the energy range 3-50 GeV(5-50 GeV). The neutrino cross section has been measured with a precision of $3 \%$ above $20 \mathrm{GeV}$ to $5 \%$ down to energies of $4 \mathrm{GeV}$. The precision on the antineutrino cross section is $9 \%$ at $5 \mathrm{GeV}$, decreases to $4 \%$ in the $15-25 \mathrm{GeV}$ region and increases again (due to statistical uncertainties) to $8 \%$ around $50 \mathrm{GeV}$. We have for the first time used the low- $v$ method to extract the neutrino and anti-neutrino fluxes in the below-30 GeV region down to energies of 3 $\mathrm{GeV}$. The neutrino and the antineutrino data in the low energy region have improved the precision of measurements in the low energy region and can be used to understand the non-scaling behavior of the cross section in this energy range. 


\section{Bibliography}

[1] ANSYS Inc., Engineering commercial software, Southpointe 275 Technology Drive, Canonsburg, PA 15317.

[2] V. Ammosov. Talk given at the first international workshop on neutrino-nucleus inter-actions in the few-gev region. NuINT01, 2001.

[3] C. Amsler. Physics Letters B, 1:667, 2008.

[4] D.Y. Bardin and V.A. Dokuchaeva. On the radiative corrections to the neutrino deep inelastic scattering. Preprint JINR-E2-86-260, 1986.

[5] A. Bodek, I. Park, and U.K. Yang. Improved low q2 model for neutrino and electron nucleon cross-sections in few gev region. arXive:hep-ph/0411202 v1, 16 Nov 2004.

[6] G. Bower and R. Cassel. unpublished version of the gheisha code with bug fixes.

[7] R. Brun. Geant detector description and simulation tool. In CERN Program Library, Long Writeup W5013, 1994.

[8] H. Budd, A. Bodek, and J. Arrington. A new parametrization of the nucleon elastic form factors. hep-ex,0602017, 2005.

[9] J. Conrad, M. H. Shaevitz, and T. Bolton. Precision measurement with high energy neutrino beams. Rev. Mod. Phys., 70:1341, 1998.

[10] A.S. Carroll et al. Pion-nucleus total cross sections in the $(3,3)$ resonance region. Phys. Rev. C, 14:635-638, 1976.

[11] A.S. Vovenko et al. Not known. Soviet J Nucl. Phys., 30:527, 1979.

[12] C. Baltay et al. Cross sections and scaling variable distributions of neutral current and charged current neutrino-nucleon interactions from a low energy narrow band beam. Physical Review Letter, 44(14):916-919, 1980.

[13] D. Ashery et al. True absorption and scattering of pions on nuclei. Phys. Rev. C, 23:2173$2185,1981$. 
[14] D.C. Colley et al. Cross sections for charged current neutrino and antineutrino interactions in the energy range 10 to 50 gev. Z. Phys., C2:187, 1979.

[15] D.G. Michael et al. The magnetized steel and scintillator calorimeters of the minos experiment. Nuclear Instruments and Methods in Physics Research A, 596:190, 2008.

[16] D.S. Baranov et al. mu+e pair production in an neutrino experiment with the bubble chamber skat. Physics Letters, 81B(2):255, 1979.

[17] J. Morfin et al. Total cross sections and nucleon structure functions in the gargamelle sps neutrino antineutrino experiment. Phys. Lett., 104B(3):235-238, 1981.

[18] J.V. Allaby et al. Total cross sections of charged-current neutrino and antineutrino interactions on isoscalar nuclei. Z Physics C - Particles and Fields, 38:403-410, 1988.

[19] K. Hagiwara et al. Review of particle properties. Phys. Rev. D, 66(010001), 2002.

[20] M. Tzanov et al. Precise measurement of neutrino and antineutrino differential cross-sections. Phys. Rev. D, 2006.

[21] N.J. Baker et al. Total cross sections for neutrino neutron and neutrino proton charged-current interactions in the 7-foot bubble chamber. Physical Review D, 25:617-623, 1982.

[22] N.J.Baker et al. Quasielastic neutrino scattering: A measurement of the weak nucleon axialvector form factor. Phys. Rev. D, 23:2499, 1981.

[23] O. Erriquez et al. Antineutrino-nucleon total cross section and ratio of antineutrino cross section on neutrons and protons. Phys. Lett., 84B:309, 1979.

[24] P. Adamson et al. A study of muon neutrino disappearance using the fermilab main injector neutrino beam. Phys. Rev. D, D77:072002, 2008.

[25] P. Berge et al. Total neurino and antineutrino charged current cross-section measurements in 100,160, and 200 gev narrow band beams. Z Physics $C$ - Particles and Fields, 35:443-452, 1987.

[26] P.S. Auchincloss et al. Measurement of the inclusive charged-current cross section for neutrino and antineutrino scattering on isoscalar nucleons. $Z$ Physics $C$-Particles and Fields, 48:411-431, 1990.

[27] Q. Wu et al. A precise measurement of the muon neutrino-nucleon inclusive charged current cross-section off an isoscalar target in the energy range $2.5<\mathrm{enu}<40 \mathrm{gev}$ by nomad. Physics Letter B, 2007.

[28] S. Campolillo et al. Total cross sectionfor neutrino charged current interactions at 3 gev and 9 gev. Physics Letters, 84B:281-284, 1979. 
[29] S.J. Barish et al. Study of neutrino interactions in hydrogen and deuterium: Description of the experiment and study of the reaction nu+d $->$ mu- + p+ps. Phys. Rev. D, 16:3103-3121, 1977.

[30] S.J. Barish et al. Study of neutrino interactions in hydrogen and deuterium. Physical Review D, 19:2521-2542, 1979.

[31] V.B. Anikeev et al. Total cross section measurements for numu, numubar charged current interactions in 3-30 gev energyrange with ihep-jinr neutrino detector. $Z$ Physics $C, 70: 39$, 1996.

[32] W.A. Mann et al. Study of the reaction nu+n -> mu- +p. Phys. Rev. Letter, 31:844, 1973.

[33] A. Fasso, A. Ferrari, J. Ranft, and P.R. Sala. Fluka: Present status and future developments. In Given at 4th International Conference on Calorimetry in High Energy Physics, pages 19-25, La Biodola, Italy, Sep 1993.

[34] H. Fesefeldt. The simulation of hadronic showers: Physics and Applications. CERN, 1985.

[35] R.P. Feynman. Very high-energy collision of hadrons. Phys. rev. Letter, 23:1415, 1969.

[36] R.P. Feynman, M. Kislinger, and F. Ravndal. Current matrix elements from a relativistic quark model. Phy. Rev., D3:2706, 1971.

[37] H. Gallagher. The neugen neutrino event generator. Nucl. Phys. B Proc. Suppl., 112:188, 2002.

[38] H. Georgi and H.D. Politzer. Freedom at moderate energies: Masses in color dynamics. Phys. Rev. D, 14:1829, 1976.

[39] M. Gluck, E. Reya, and A. Vogt. Dynamical parton distributions revisited. Eur. Phys. J, C5:461, 1998.

[40] R. Gran and E. J. Jeon et al. Measurement of the quasielastic axial vector mass in neutrino interactions on oxygen. Phys. Rev. D, 74:052002, 2006.

[41] D.E. Groom, N.V. Mokhov, and S.I. Striganov. Muon stopping-power and range tables: 10mev - 100 tev. Atom. Data Nucl. Data Tabl., 78:183, 2001.

[42] Z. Koba, H. B. Nielsen, and P. Olesen. Scaling of multiplicity distributions in high-energy hadron collisions. Nucl. Phys. B, 40:317, 1972.

[43] Michael Kordosky. Hadronic interactions in the MINOS detectors. PhD thesis, University of Texas, Austin, 2004.

[44] Michael Kordosky. Intranuke reweighting update at ely 2007. MINOS doc-3236, june 2007.

[45] Konstantin S. Kuzmin, Vladimir V. Lyubushkin, and Vadim A. Naumov. Nuclear effects in neutrino interactions. Acta Phys.Polon.B, B37:2337-2348, 2006. 
[46] D.B. Macfarlane. Nucleon structure functions from high energy neutrino interactions with iron and qcd results. Z Physics $C$ - Particles and Fields, 26:1-12, 1984.

[47] John Marshall. A new implementation of the kalman filter. MINOS doc-1248, nov 2005.

[48] John Marshall. A new track finding package. MINOS doc-2104, july 2006.

[49] R. Merenyi. Determination of pion intranucelar rescattering rates in muon-neutrino ne versus muon-neutrino d interactions for the atmospheric neutrino flux. Phys. Rev. D, 45:743-751, 1992.

[50] James Musser. A simple model for relating momentum and field scale shifts. MINOS doc3220 , june 2007.

[51] Thomas Henry Osiecki. A Search for Sterile Neutrinos in MINOS. PhD thesis, University of Texas, Austin, 2007.

[52] Rustem Ospanov. A measurement of muon neutrino disappearance with the MINOS detectors and the NUMI beam. PhD thesis, University of Texas, Austin, 2008.

[53] Donald H. Perkins. Introduction to high energy physics. Fourth edition, section 5.7.2, 2000.

[54] D. Rein and L. Sehgal. Neutrino-excitation of baryon resonances and single pion production. Ann. Physics, 133:79, 1981.

[55] W. Seligman. A Next-to-leading order QCD analysis of neutrino - iron structure functions at the Tevatron. PhD thesis, Columbia University, 1996.

[56] C.H. Llewellyn Smith. Neutrino reactions at accelerator energies. Phys. Rep., 3C:261, 1971.

[57] M.R. Whaley. Durham database. Nucl. Phys Proc. Suppl., 139:241, 2005.

[58] E.J . Wolin and L.L. Ho. Covariance matrices for track fitting with the kalman filter. Nuclear Instruments and Methods in Physics Research A, 329:493-500, 1993.

[59] F.J. Yndurain. The Theory of Quark and Gluon Interaction. Springer Verlag, 1993.

[60] C. Zeitnitz and T. A. Gabriel. The geant-calor interface and benchmark calculations of zeus test calorimeters. Nucl. Intruments and Methods, A349:106, 1994.

[61] Robert Zwaska. Accelerator Systems and Instrumentation for the NuMI Neutrino Beam. PhD thesis, University of Texas, Austin, 2006. 


\section{APPENDIX A}

\section{KALMAN FILTER}

The Kalman filter $[47,58]$ uses a $\mathrm{p} \times 1$ dimensional matrix known as the state vector and a model that extrapolates this state vector to the subsequent planes. The state vector $\mathbf{x}_{k}$ in the k-th plane can be written as

$$
\mathbf{x}_{k}=\mathbf{F}_{k-1} \mathbf{x}_{k-1}+\mathbf{w}_{k-1}
$$

where $\mathbf{F}_{k-1}$ is the $\mathrm{p} \times \mathrm{p}$ propagator matrix that extrapolates the state vector from the (k-1)th plane to the k-th plane and $\mathbf{w}_{k-1}$ is the "process noise" from multiple Coulomb scattering and ionization energy loss. The actual measurement(observed value) in plane $\mathrm{k}$ is denoted by a $\mathrm{m} \times 1$ vector $\mathbf{m}_{k}$ that is given by

$$
\mathbf{m}_{k}=\mathbf{H}_{k} \mathbf{x}_{k}+\varepsilon_{k}
$$

$\mathbf{H}_{k}$ is a $\mathrm{m} \times \mathrm{p}$ dimensional matrix that is also known as the measurement function and $\varepsilon_{k}$ is the "measurement noise" or measurement errors. Both the process noise $\left(\mathbf{w}_{k}\right)$ and the measurement noise $\left(\varepsilon_{k}\right)$ are assumed to be independent of each other and normally distribued with a zero mean.

In the Kalman technique, $\widehat{\mathbf{x}}_{k}^{s}$ is defined as the best "estimate"(expectation value) of the state vector $\mathbf{x}_{k}$ including all measurement up to and including the plane s. An expression for $\widehat{\mathbf{x}}_{k}^{k-1}$ can be derived from Eq. A.1

$$
\mathbf{x}_{k}\left|\mathbf{m}_{k-1}=\mathbf{F}_{k-1} \mathbf{x}_{k-1}\right| \mathbf{m}_{k-1}+\mathbf{w}_{k-1} \mid \mathbf{m}_{k-1}
$$




$$
=\mathbf{F}_{k-1} \mathbf{x}_{k-1} \mid \mathbf{m}_{k-1}+\mathbf{w}_{k-1} .
$$

Taking the expectation value of both sides of Eq. A.3 $(\widehat{\mathbf{w}} \sim 0)$

$$
\widehat{\mathbf{x}}_{k}^{k-1}=\mathbf{F}_{k-1} \widehat{\mathbf{x}}_{k-1}^{k-1}
$$

$\widehat{\mathbf{x}}_{k}^{k}$ is determined by

$$
\begin{aligned}
\widehat{\mathbf{x}}_{k}^{k} & =\widehat{\mathbf{x}}_{k}^{k-1}+\mathbf{K}_{k}\left(\mathbf{m}_{k}-\mathbf{H}_{k} \widehat{\mathbf{x}}_{k}^{k-1}\right) \\
& =\mathbf{F}_{k-1} \widehat{\mathbf{x}}_{k-1}^{k-1}+\mathbf{K}_{k}\left(\mathbf{m}_{k}-\mathbf{H}_{k} \mathbf{F}_{k-1} \widehat{\mathbf{x}}_{k-1}^{k-1}\right) .
\end{aligned}
$$

The $\mathrm{p} \times \mathrm{m}$ matrix $K$ is the Kalman gain matrix that is derived from the covariance matrices. $\widehat{\mathbf{x}}_{k}^{k}$ is referred to as the predicted estimate of the state vector $\mathbf{x}_{k}$ and $\widehat{\mathbf{x}}_{k}^{k-1}$ is referred to as the filtered estimate of the state vector $\mathbf{x}_{k}$.

The covariance matrix $\mathbf{C}_{k}^{k}$ of the state vector $\mathbf{x}_{k}$ is expressed as

$$
\mathbf{C}_{k}^{k}=E\left[\left(\mathbf{x}_{k}-\widehat{\mathbf{x}}_{k}^{k}\right)\left(\mathbf{x}_{k}-\widehat{\mathbf{x}}_{k}^{k}\right)^{T}\right]
$$

where $E$ is the expectation value. By substituting Eq. A.5 for $\widehat{\mathbf{x}}_{k}^{k}, \mathbf{C}_{k}^{k}$ can be written as

$$
\mathbf{C}_{k}^{k}=\mathbf{C}_{k}^{k-1}-\mathbf{K}_{k} \mathbf{H}_{k} \mathbf{C}_{k}^{k-1}-\mathbf{C}_{k}^{k-1} \mathbf{H}_{k}^{T} \mathbf{K}_{k}^{T}+\mathbf{K}_{k}\left(\mathbf{H}_{k} \mathbf{C}_{k}^{k-1} \mathbf{H}_{k}^{T}+\mathbf{V}_{k}\right) \mathbf{K}_{k}^{T}
$$

The Kalman gain is estimated by minimizing the square error estimator which is given by $E[\mid \mathbf{x}-$ $\left.\left.\widehat{\mathbf{x}}_{k}^{k}\right|^{2}\right]$. This is equivalent to minimizing the trace of the covariance matrix $\mathbf{C}_{k}^{k}$

$$
\frac{\partial \operatorname{tr}\left(\mathbf{C}_{k}^{k}\right)}{\partial \mathbf{K}_{k}}=-2\left(\mathbf{H}_{k} \mathbf{C}_{k}^{k-1}\right)^{T}+2 \mathbf{K}_{k}\left(\mathbf{H}_{k} \mathbf{C}_{k}^{k-1} \mathbf{H}_{k}^{T}+\mathbf{V}_{k}\right)=0
$$

Solving for $\mathbf{K}_{\mathbf{k}}$ gives

$$
\mathbf{K}_{k}=\mathbf{C}_{k}^{k-1} H_{k}^{T}\left(\mathbf{H}_{k} \mathbf{C}_{k}^{k-1} \mathbf{H}_{k}^{T}+V_{k}\right)^{-1} .
$$

Eq. A.7 can be simplified by substituting Eq. 1.13

$$
\mathbf{C}_{k}^{k}=\left(1-\mathbf{K}_{k} \mathbf{H}_{k}\right) \mathbf{C}_{k}^{k-1}
$$




\section{APPENDIX B \\ CORRECTIONS TO DIS CROSS SECTION MODEL}

The differential equation used in the current code has an inconsistency, the structure functions are calculated at $x=\xi_{W}$ but while calculating the cross section using Eq. 1.31 it uses $x$. This mistake mainly affects the low $x$ values because that is the region where $x$ and $\xi_{W}$ differ significantly.

In the present model by Bodek and Yang $2 x F_{1}$ is calculated by using

$$
2 x F_{1}\left(x, Q^{2}\right)=F_{2}\left(\frac{1+\frac{4 M^{2} x^{2}}{Q_{\min }^{2}}}{1+R_{L}\left(x, Q^{2}\right)}\right) .
$$

To prevent $2 x F_{1}$ from approaching infinity at low $Q^{2}$, a minimum $Q^{2}\left(0.8 \mathrm{GeV}^{2}\right)$ value is used in the numerator. This is a temporary solution and a more proper approach is to apply a minimum $v$ and $W$ threshold to the present model ${ }^{1}$. In the corrected model, Bodek-Yang parametrization is applicable only for $v$ greater than $0.134 \mathrm{GeV}$ and $W$ greater than $1.7 \mathrm{GeV}$. Below this value, the effective rescaling variable $\xi_{W}$ will be replaced by $x$ and $2 x F_{1}$ will be equal to $F_{2}$. These mistakes will be corrected in the next version of the cross section model.

Fig. B1 shows the effect of these corrections on the ratio of $\sigma(\bar{v}) / \sigma(v)$. After applying the corrections, the predicted value agrees better with the average value of this ratio between 30-200 $\mathrm{GeV}$ from experiments.

In addition to the changes mentioned above, there is another difference between the cross section model proposed by Bodek-Yang and the default model used in MINOS. The Bodek-Yang model takes into account only one resonance channel $(\Delta(1232))$ below $\mathrm{W}$ of $1.7 \mathrm{GeV}$ but in the

\footnotetext{
${ }^{1}$ Personal communication with Arie Bodek
} 
default NEUGEN3 model, contribution from 16 resonances have been considered. Fig. B2 and B3 shows the effect on the low- $v$ correction caused by these three changes. The change in low- $v$ correction is of the order of $1 \%$ or smaller. 


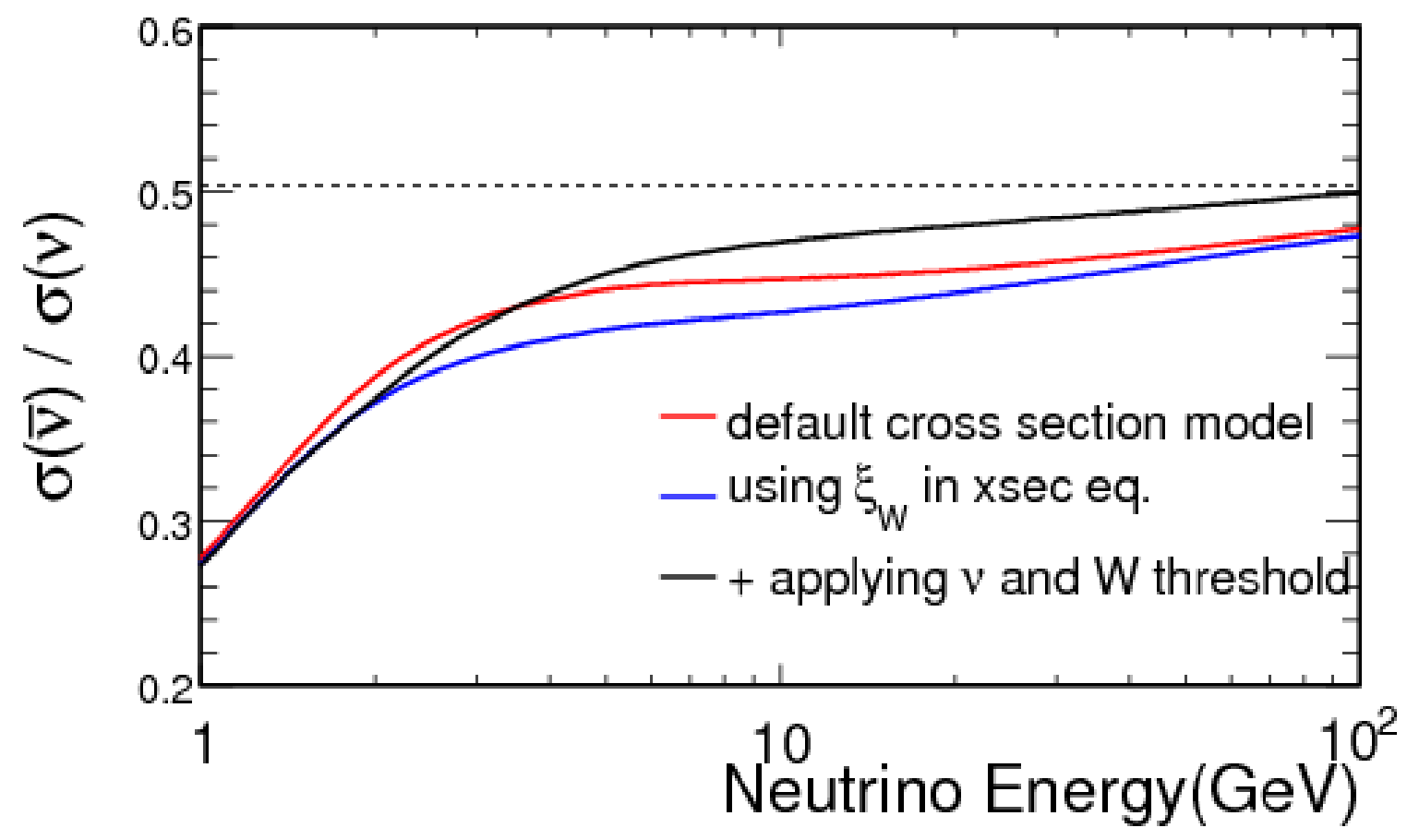

Figure B1: Ratio of $\sigma(\bar{v}) / \sigma(v)$ from the NEUGEN cross section model. The red line shows the default ratio, the blue line shows the ratio after using $\xi_{W}$ in the cross section equation and the red line shows the ratio after applying a $v$ and $\mathrm{W}$ threshold. The dashed line shows the average value of this ratio between $30-200 \mathrm{GeV}$ from experiments. 

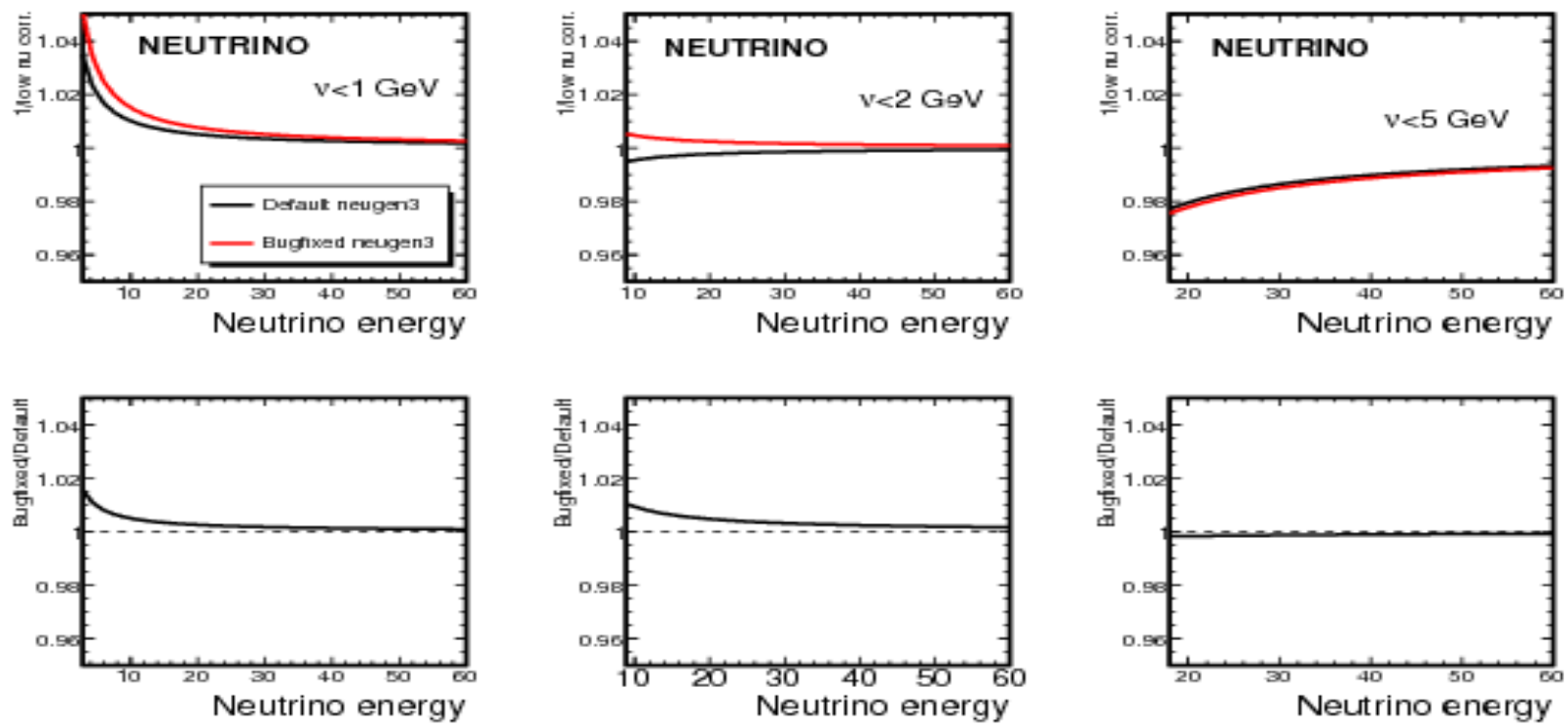

Figure B2: Comparison of neutrino low $-v$ correction calculated from the default cross section model(NEUGEN3) and the corrected model.
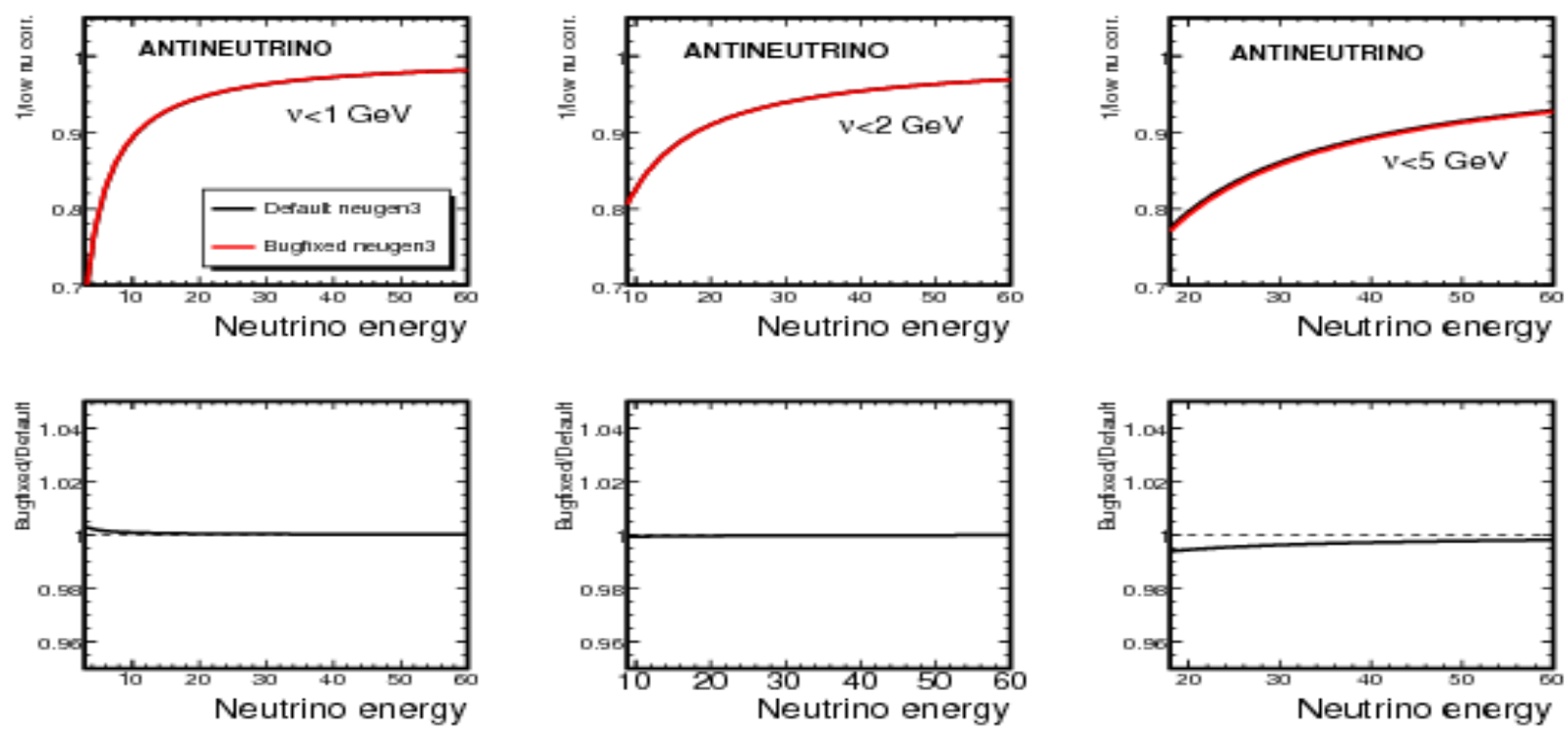

Figure B3: Comparison of antineutrino low- $v$ correction calculated from the default cross section model(NEUGEN3) and the corrected model. 


\section{APPENDIX C}

\section{COMPARISON OF FLUX TUNING TECHNIQUES}

The procedure for tuning the default GNUMI neutrino flux using the low- $v$ event sample has been described in section 7.2. A different method was employed to tune the input flux that involved fitting the secondary hadron production simulation model to the Near Detector cross section data [24]. This method is referred to as the "SKZP tuning". This section presents a comparison of the weighting technique using these two different methods.

Fig. C1 shows the comparison of low- $v$ and SKZP weights for the neutrino sample. The radiative correction has been removed from the low- $v$ sample for this study because these corrections were not applied in the SKZP reweighting procedure. In general, at energies below $30 \mathrm{GeV}$ the low- $v$ weights are about $5 \%$ smaller than the SKZP weights and at energies above $30 \mathrm{GeV}$, the low- $v$ weights are $5 \%$ larger than the SKZP weights. The two weights agree with each other at the level of $3 \%$ within error bars.

Fig. C2 shows the comparison of low- $v$ and SKZP weights for the antineutrino sample. The weights overlap within error bars but there is an offset of the order of 5-10\%. The offset at high energy $(>25 \mathrm{GeV})$ can be understood by looking at Fig. C3 that shows the data and MC comparison plot of the neutrino energy for flux sample(or the low- $v$ sample) and the cross section sample. There is clearly an offset in data and MC agreement for the two samples at the level of $\sim 5 \%$ above $25 \mathrm{GeV}$. The SKZP flux was tuned to the cross section sample that explains the offset between the low- $v$ weight and SKZP weight. The offset at low energy is more difficult to understand. The low- $v$ antineutrino sample uses only the relatively high energy exiting downstream sample (see section [ref]) whereas the SKZP technique uses the low energy samples as well. The low energy 
sample is expected to have a larger data and MC disagreement because of higher wrong-sign and NC contamination, which implies a larger SKZP weight. This effect could be causing the offset between the two weights seen at low energy. 

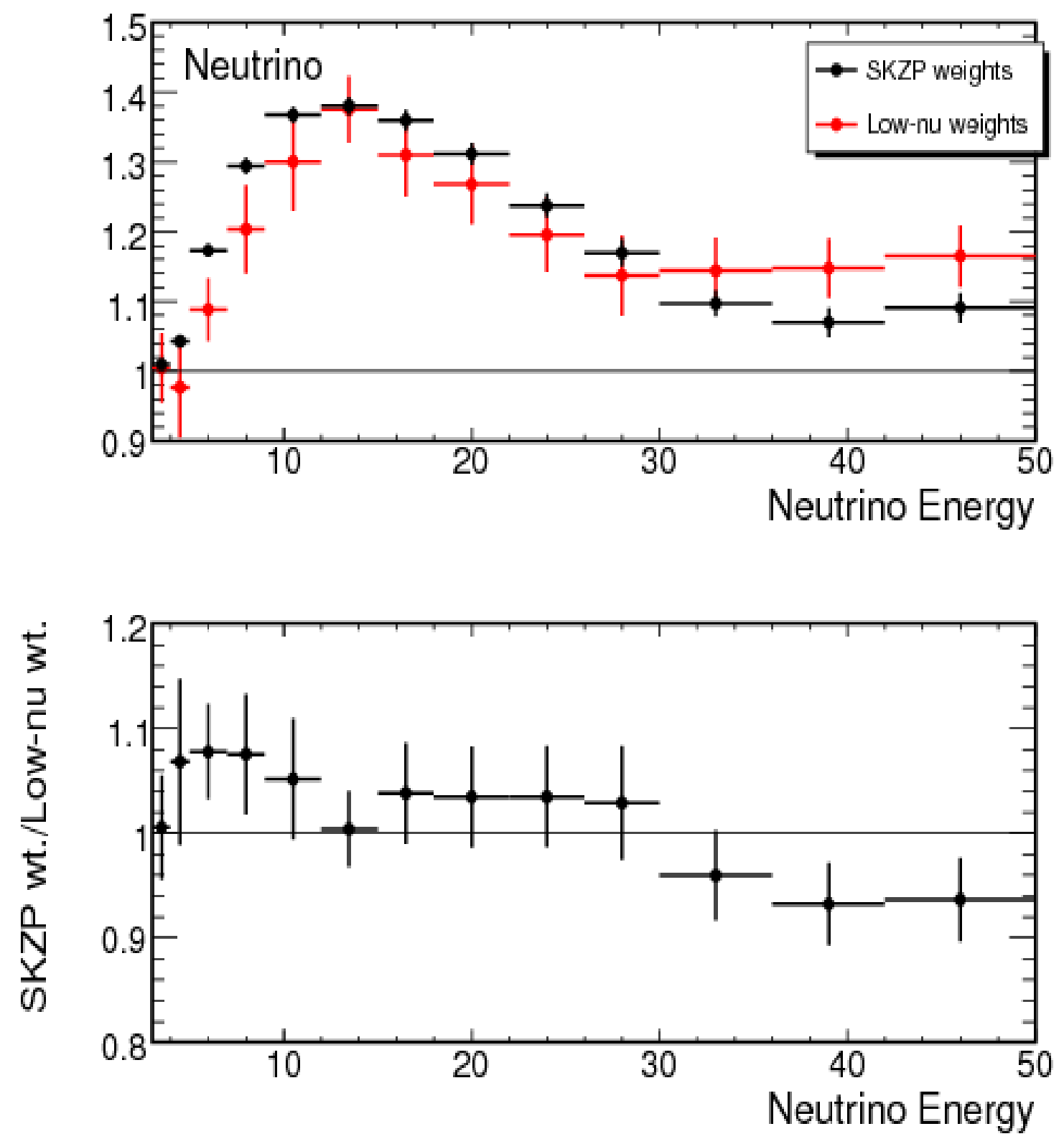

Figure C1: Comparison of flux tuning using the low- $v$ and SKZP method for the neutrino sample. The bottom plot shows the ratio of these two weights. The low- $v$ weights have statistical and systematic uncertainties added in quadrature, the SKZP weights have only the statistical uncertainties. 

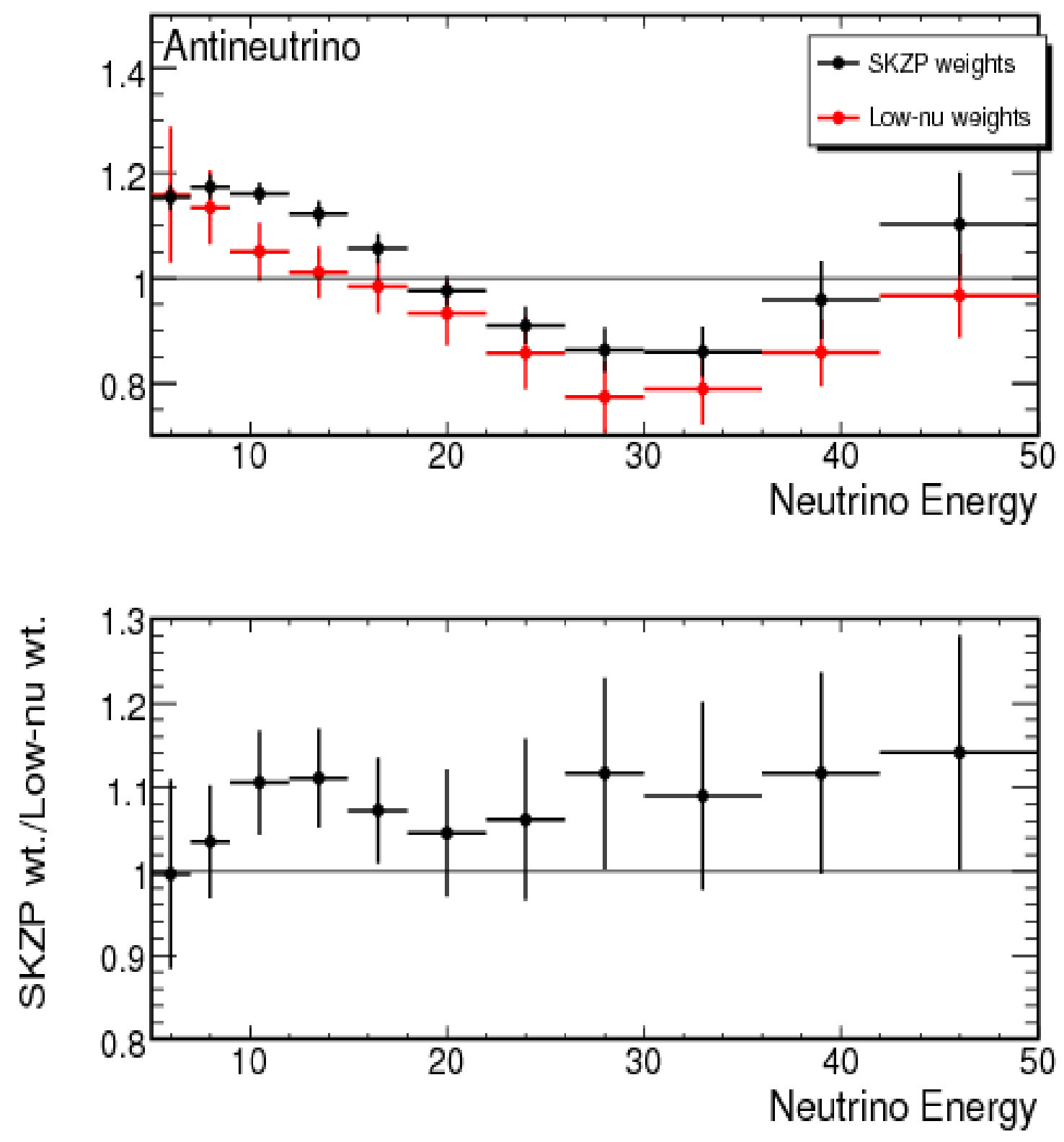

Figure C2: Comparison of flux tuning using the low- $v$ and SKZP method for the antineutrino sample. The bottom plot shows the ratio of these two weights. The low- $v$ weights have statistical and systematic uncertainties added in quadrature, the SKZP weights have only the statistical uncertainties. 

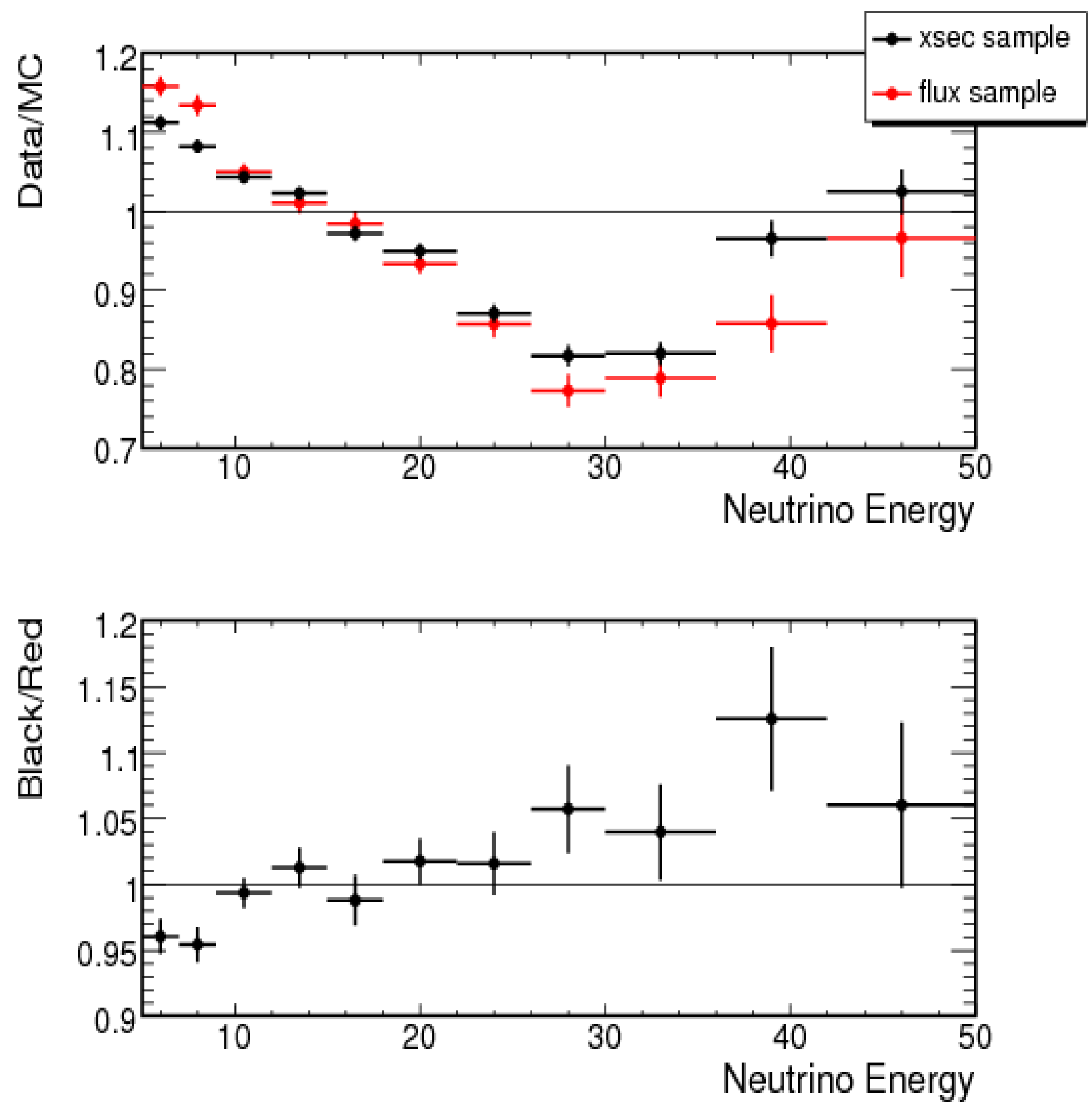

Figure C3: Comparison of data and MC simulation. The bottom plot shows the ratio of these two weights. The low- $v$ weights have statistical and systematic uncertainties added in quadrature, the SKZP weights have only the statistical uncertainties. 


\section{APPENDIX D}

\section{$Q^{2}$ RESOLUTION}

$Q^{2}$ resolution plots for a Deep Inelastic Scattering enhanced sample is presented in this section. This sample is selected by applying $E_{V}>5 \mathrm{GeV}, E_{S H W}>1 \mathrm{GeV}$ and $Q^{2}>1 \mathrm{GeV}^{2}$ selection cuts to the CC sample (section 6.1). Fig. D1 shows the distribution of the difference of reconstructed and true $Q^{2}$ in four true $Q^{2}$ bins. The plot shows that as the value of $Q^{2}$ increases, the width of the distribution increases and the resolution detoriates. Fig. D2 shows that $Q^{2}$ increases with $\theta_{\mu}$. It has been previously shown in Fig. 4.7 that the resolution gets worse with increasing $\theta_{\mu}$. The

worsening $Q^{2}$ resolution with increasing $Q^{2}$ can be attributed to the fact that the resolution of $\theta_{\mu}$ worsens with increasing angle. 

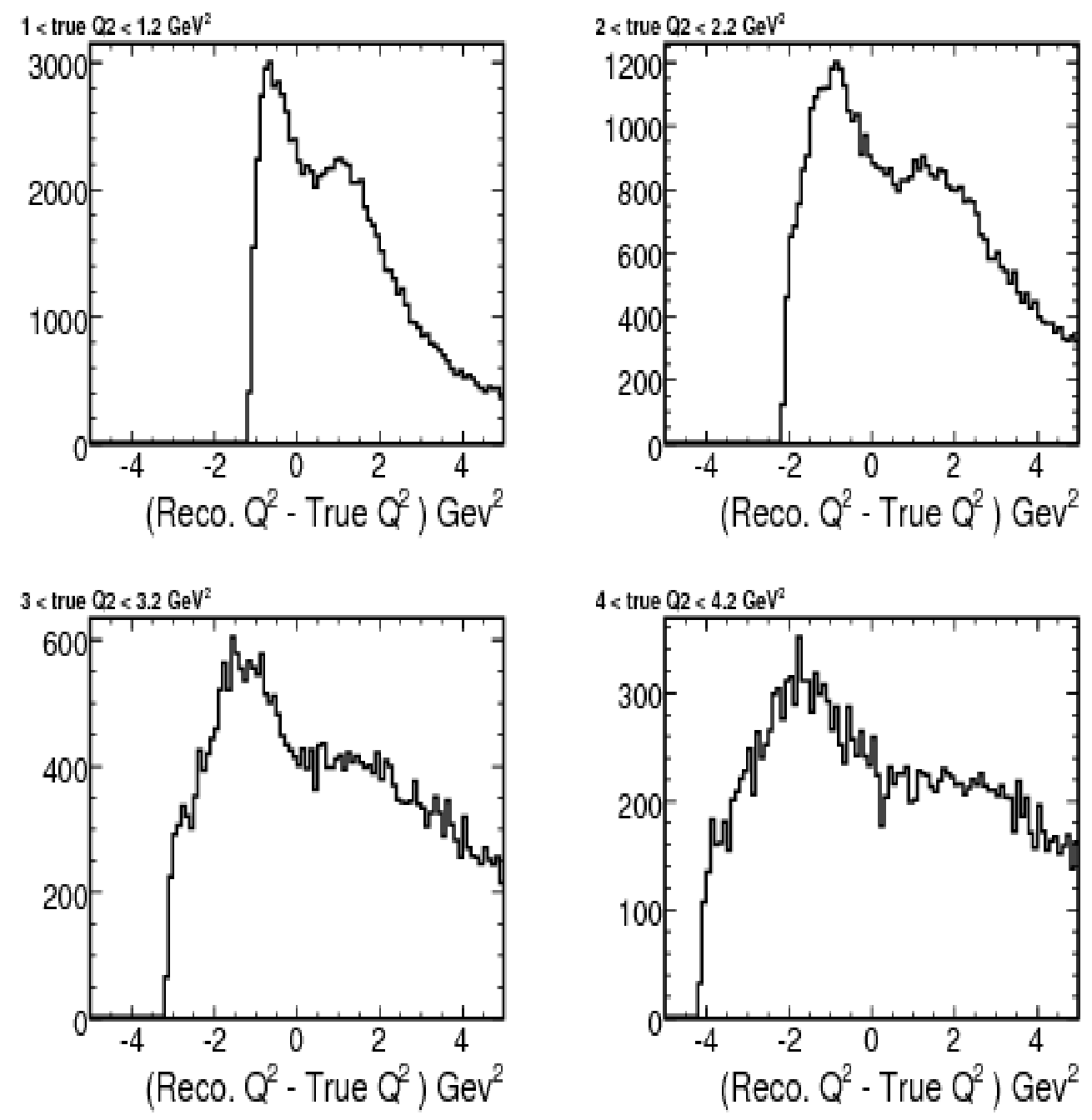

Figure D1: Distribution of (reconstructed $Q^{2}$ - true $Q^{2}$ ) for a neutrino DIS selected sample in four true $Q^{2}$ bins. 


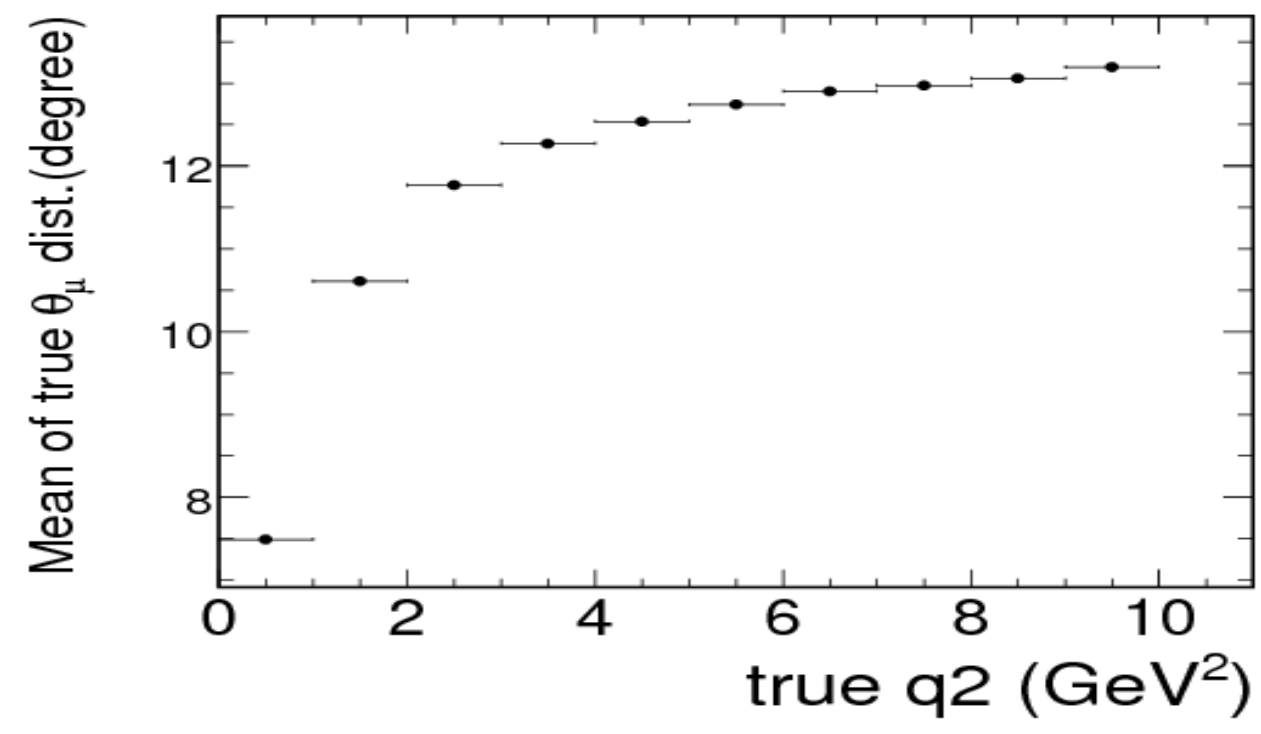

Figure D2: Mean of true angle distribution as a function of true $Q^{2}$ for the neutrino enhanced DIS sample. 


\section{APPENDIX E}

\section{MINOS COLLABORATION}

1. Argonne National Laboratory D.S. Ayres, W.L. Barrett, T.H. Fields, M.C. Goodman, X. Huang, A.M. McGowan, J. Reichenbacher, D.E. Reyna, M.C. Sanchez, R.L. Talaga, J.L. Thron

2. University of Athens D. Drakoulakos10, P. Stamoulis, G. Tzanakos, M. Zois

3. Benedictine University M.V. Frohne, P. Schreiner

4. Brookhaven National Laboratory M. Bishai, M. Dierckxsens, M.V. Diwan, D.E. Jaffe, B. Viren, Z. Wang, L. Whitehead, K. Zhang

5. California Institute of Technology B. Barish, A. Himmel, C. Howcroft, D.G. Michael, L. Mualem, H.B. Newman, J.P. Ochoa-Ricoux, M. Orchanian, R.B. Patterson, C.W. Peck, H. Zheng

6. University of Cambridge A. Blake, J.D. Chapman, A.J. Culling, J.S. Marshall, J. Mitchell, M.A. Thomson, R. Toner, C.P. Ward, D.R. Ward

7. Fermi National Accelerator Laboratory P. Adamson, B. Baller, R.H. Bernstein, G.J. Bock, D.J. Boehnlein, D. Bogert, E. Buckley-Geer, S. Childress, B.C. Choudhary,R. Ford, N. Grossman, D. Harris, R. Hatcher, J. Hylen, C. James, D. Jensen, G. Koizumi, A. Kreymer, P. Lucas, A. Marchionni, C.D. Moore, J.G. Morfin, A. Para, R.K. Plunkett, R.A. Rameika, B. Rebel, N. Saoulidou, P. Shanahan, W. Smart, A. Wehmann, R. Zwaska

8. Harvard University J. Boehm, S. Cavanaugh, G.J. Feldman

9. Illinois Institute of Technology J.K. de Jong, H.A. Rubin, C. White 
10. Indiana University R. Armstrong, C. Bower, M. Ishitsuka, N. Mayer, M.D. Messier, S. Mufson, J.A. Musser, J. Paley, J. Urheim University College London M. Dorman, J.J. Evans, A. Holin, D.J. Koskinen, R.J. Nichol, R. Saakyan, C. Smith, J. Thomas

11. University of Minnesota, Twin Cities K.E. Arms, B.R. Becker, P.M. Border, D. CroninHennessy, J. Gogos, E.W. Grashorn, K. Heller, S.M.S. Kasahara, Z. Krahn, S. Kumaratunga, P.J. Litchfield, M.L. Marshak, J.R. Meier, W.H. Miller, E.A. Peterson, D.A. Petyt, D. Rahman, K. Ruddick, B. Speakman, M. Strait

12. University of Minnesota, Duluth B. Bock, R. Gran, A. Habig, A. Mislivec

13. Otterbein College N. Tagg

14. University of Oxford C. Backhouse, G. Barr, J.H. Cobb, R.P. Litchfield, R. Pittam, P.A. Rodrigues, A. Sousa, G. Tinti, A. Weber, N. West

15. University of Pittsburgh D. Bhattacharya, I.Z. Danko, S.A. Dytman, Z. Isvan, M.S. Kim, D. Naples, V. Paolone

16. Rutherford Appleton Lab C. Andreopoulos, A. Belias, T. Durkin, C.J. Metelko, T.C. Nicholls, G.F. Pearce, T.M. Raufer

17. University of South Carolina A. Godley, J.J. Kim, J. Ling, S.R. Mishra, A. Rahaman, C. Rosenfeld

18. Stanford University G.M. Irwin, (H.J. Kang(4/09), (S. Murgia(3/10),) G. Pawloski, S.G. Wojcicki, T. Yang University of Sussex D.J. Auty, N.E. Devenish(2/08), E. Falk Harris, (P.G. Harris(5/15), ) J. Hartnell, M.A. Tavera

19. Texas A\&M University (E. Tetteh-Lartey(9/11),) M. Watabe, R.C. Webb

20. University of Texas at Austin K. Bain(1/08), J.P. Cravens(9/08), (D. Indurthy(3/08), S. Kopp, K. Lang, L. Loiacono, J. Ma, R. Mehdiyev(11/07), (T. Osiecki(3/10),) R. Ospanov, (Z. Pavlovic(6/10))

21. Tufts University D. Cherdack, H.R. Gallagher, T. Kafka, W.A. Mann, (R.H. Milburn(12/08),) W.P. Oliver, J. Schneps

22. UNICAMP - University of Campinas J. Coelho(6/08), C.O. Escobar

23. USP - University of Sao Paulo P. Gouffon

24. Warsaw K. Grzelak

25. William \& Mary S.J. Coleman, M. Kordosky, J.K. Nelson, P. Vahle 